\title{
Ökonomische Beiträge zur Erschließung menschlichen Verhaltens für die Lösung von Naturschutzfragen
}

\author{
Dissertation \\ zur Erlangung des Doktorgrades \\ der Fakultät für Agrarwissenschaften \\ der Georg-August-Universität Göttingen
}

\author{
vorgelegt von \\ Jan Michael Freese \\ geboren in Sande
}

Göttingen, November 2006 
D7

1. Referent: Prof. R. Marggraf

2. Korreferent: Prof. L. Theuvsen

Tag der mündlichen Prüfung: 16.11.2006 


\section{Inhaltsverzeichnis}

Zusammenfassung 8

Einleitung 10

Umwelt- und Naturschutz als Untersuchungsgegenstand 11

Untersuchungskonzept 12

Literatur 16

Kapitel 1 Bausteine einer rationalen ökonomischen Rekonstruktion sozialer Interaktionen. Warum auch ökonomische Modellindividuen sozial interagieren und öffentliche Güter erstellen _17

1.1. Einleitung 17

1.2. Allmende-, Umwelt- und öffentliche Güter 18

1.3. Öffentliche Güter in der ökonomischen Theorie 20

1.3.1. Von der Ein-Individuen- zur Mehr-Individuen-Ökonomie 21

1.3.2. Lösungsstrategien 23

1.4. Die erweiterte Kapitaltheorie: Human- und Sozialkapital 26

1.5. Das Konzept des Sozialkapitals von Coleman 32

1.6. Soziologische Austauschtheorien 33

1.6.1. Bereitstellung öffentlicher Güter im Austausch gegen soziale Anerkennung 34

1.7. Erweiterung des Kapitalmodells durch Identitätskapital und Distinktionsnutzen 36

1.7.1. Psychologische Konzepte von Identität 37

1.7.1.1. Identitätsdiskurse

1.7.1.2 Die Postmoderne

1.7.1.3. Das postmoderne Identitätsverständnis

1.7.2. Ein funktionales Modell sozialer Interaktionen und das Konzept des Distinktionsnutzens 42

1.7.3. Identitätskapital 46

1.7.3.1. Identität und Ökonomie

1.7.3.2. Eigenschaften des Identitätskapitals 46

1.7.3.3. Auswirkungen sozialer Interaktionen auf die Nutzenfunktion

1.8. Identitätskapital, Distinktionsnutzen und öffentliche Güter 52

1.8.1. Entstehung des Sozialen 52

1.8.2. Entstehung öffentlicher Güter bei Berücksichtigung des Distinktionsnutzens 1.8.2.1. Das klassische Dilemma

1.8.2.2. Kooperativer Nutzer mit Identitätskapitalzuwachs

1.8.2.3. Öffentlicher egoistischer Nutzer mit Identitätskapitalzuwachs und sozialen Kosten 55 
1.9.1. Distinktionsnutzen und Identitätskapital in verschiedenen Lebenssituationen 58

1.9.2. Nutzen einer sozialen Interaktion in Abhängigkeit von der sozialen Nähe zum Interaktionspartner 59

1.10. Fazit: Identitätskapital und Distinktionsnutzen als konstitutive Merkmale eines ökonomischen Individuums 60

1.11. Anhang 64

1.11.1. Die monetarisierte Auszahlungsfunktion eines öffentlichen Gutes 64

1.12. Literatur 65

2.1. Die Konfliktlage zwischen Landwirtschaft und Naturschutz 71

2.2. Psychologische Hintergründe des Konflikts 73

2.3. Drei Blickwinkel von Partizipation 74

2.3.1. Mitgestaltung der Landnutzung durch den Naturschutz 74

2.3.2. Beteiligung der Landwirte an der Ausgestaltung des Naturschutzes 77

2.3.3. Bevölkerungsbeteiligung 79

2.4. Regionale Partnerschaft für den Naturschutz in der Agrar- und Kulturlandschaft _ 83

2.5. Fazit 85

2.6. Literatur 86

\section{Kapitel 3 Zahlungsbereitschaftsanalysen in umweltrelevanten} Bewertungsverfahren

Die Eingriffsregelung als Beispiel 89

3.1. Einleitung 89

3.2. Die Instrumente umweltbezogener Bewertungsverfahren 90

3.3. Die Eingriffsregelung als Beispiel 92

3.3.1. Prinzip der Eingriffsregelung: Vermeidung, Ausgleich, Ersatz 92

3.3.2. Bewertungsmethoden 93

3.3.3. Weiterentwicklung der Eingriffsregelung: Ausgleichszahlungen und Monetarisierung der Eingriffsfolgen 96

3.4. Einsatzmöglichkeiten von Zahlungsbereitschaftsanalysen im Rahmen der Eingriffsregelung 99

3.4.1. Die Ermittlung gesellschaftlicher Werte 99

3.4.2. Exkurs: Zahlungsbereitschaftsanalysen als Ergänzung zu fachinternen Planungen_ 104

3.5. Fazit 105

3.6. Literatur 106 
Kapitel 4 Improving the institutional delivery of agrienvironmental schemes via local action groups

4.1. Introduction 108

4.2. Balancing AES: ecology, economics and farmers' acceptance 109

4.3. Local management organisations for the agri-environment 112

4.3.1. Different organisation forms 112

4.3.2. Experiences in a local project: The "Bördeprojekt Wolfenbüttel” 113

4.4. Discussing factors of success for AES 115

4.4.1. Usage of existing networks 115

4.4.2. A dualistic partnership with a strong “public to private” element 116

4.4.3. Dedicated management 116

4.4.4. Process competence 117

4.4.5. Flexibility matters 118

4.5. Conclusions 118

4.6. References 119

Kapitel 5 Stakeholder involvement in agri-environmental policy making - Success factors for participatory approaches from two German case studies

5.1. Introduction 123

5.2. Agri-environmental programming 125

5.2.1 Process and responsibilities 125

5.2.2. Conflicting interests 128

5.4. Two Key studies 130

5.5. Discussion of lessons learned 134

5.5.1 Premises and preparation 135

5.5.2 Structuring and facilitating the participatory process 137

5.5.3 Problems encountered 138

5.5.4 Benefits 140

5.6. Conclusions and Recommendations 143

5.7. Appendix 145

5.8. References 146 
Kapitel 6 A Fuzzy Tale - Die kleinen Leute von Borka 149

Fazit 152

Veröffentlichungen (Stand September 2006) 154

Danksagung 156

Lebenslauf 157 


\section{Ökonomische Beiträge zur Erschließung menschlichen Verhaltens für die Lösung von Naturschutzfragen}

„Vernunft heißt, ein Gleichgewicht zwischen den eigenen Wünschen und den Rechten der anderen zu finden, an alle denken zu können: an die anderen Menschen, die Nachkommen, die Natur und auch an sich selbst."

Ulrich Hampicke (1992: 8) 


\section{Ökonomische Beiträge zur Erschließung menschlichen Verhaltens für die Lösung von Naturschutzfragen}

\section{Zusammenfassung}

Ökonomische Betrachtungen liefern wichtige Beiträge zur Lösung von Naturschutzfragen, da sie sozio-ökonomische Ursachen von Naturschutzproblemen bzw. Hemmfaktoren der Umsetzung naturkonformen Verhaltens ermitteln können.

In Kapitel 1 werden anhand der erweiterten Kapitaltheorie individuelle rationale Verhaltensanreize zu sozialen Interaktionen analysiert und mittels des umweltökonomischen Konzeptes der öffentlichen Güter die darauf aufbauenden kollektiven und naturschutzrelevanten Verhaltensphänomene erklärt. Dieses Kapitel stellt das ökonomische Verständnis sozialer Interaktion vor, erweitert die bestehende Theorie um Aspekte der Identität und liefert so mit der Bereitstellung eines konsistenten rationalen Anreizes für soziale Interaktionen die Grundlage für die Betrachtung sozialer und gesellschaftlicher Phänomene, in die jedes Naturschutzproblem und jedes Naturschutzvorhaben eingebettet sind.

In Kapitel 2 werden zunächst die psychologischen Grundlagen des Konfliktes zwischen Naturschutz und Landwirtschaft vorgestellt, um dann anhand dreier Perspektiven die partizipativen Prozesse zu beleuchten, die nötig sind, um in diesem Konfliktbereich kooperative Lösungen zu ermöglichen. Neben der Partizipation von Vertretern des Naturschutzes an Prozessen und Entscheidungen im landwirtschaftlichen Bereich spielt die Integration der Landwirte und die Einbeziehung der Bürger in die Prozesse des Agrarumweltschutzes eine bedeutende Rolle, wenn der Begriff der Partizipation umfassend verstanden wird. Hierauf aufbauend betrachten Kapitel 4 und 5 die Erfolgsfaktoren konkreter kooperativer Projekte zur Entwicklung der Agrarumwelt in intensiv genutzten Ackerbauregionen und analysieren die Erfolgs- und Hemmfaktoren der Projekte und des politisch gesetzten Projektrahmens. Die Herausforderung der Agrarumweltprogramme besteht darin, dass die Landwirte als Adressaten und Umsetzer der Maßnahmen freiwillig teilnehmen und daher die ökologischen Ziele mit der Akzeptanz durch die Landwirte und der ökonomischen Effizienz der eingesetzten öffentli- 
chen Mittel in Einklang gebracht werden müssen. Kapitel 5 setzt den Schwerpunkt auf die Analyse der partizipativen Fortschritte durch Projekte, die auf unterschiedlichen Ebenen die Einbindung der Stakeholder in den Prozess des Agrarumweltschutzes erprobt haben. In Niedersachsen erfolgte die Beteiligung im Rahmen des Bördeprojektes Wolfenbüttel auf der regionalen Ebene bei der konkreten Ausgestaltung von Maßnahmen, die später vom Land in dessen Agrarumweltprogramm übernommen wurde. In Sachsen-Anhalt wurden die Stakeholder im Rahmen des SUTRA-Projektes in den Prozess der Programmsteuerung und Budgetverteilung auf Landesebene integriert.

Kapitel 3 greift die Beteiligung der Bevölkerung an einem weiteren wichtigen Naturschutzinstrument, der naturschutzfachlichen Eingriffsregelung, auf und erweitert dieses um die Zahlungsbereitschaftsanalyse als Instrument zur Berücksichtigung gesellschaftlicher (regionaler) Wertschätzung von Arten, Biotopen, Landschaftsbild sowie der Natur als Heimat, Freizeit-, Erholungs- und Lernraum.

Kapitel 6 will schließlich mit einer Parabel daran erinnern, dass den Erfolgen disziplinärer und methodischer Ansätze zum Trotz, die Ergebnisse bei der Übertragung auf die Praxis, z. B. in Form der Projekt- und Politikberatung, sorgfältig gegen andere Erkenntnisse, Bedürfnisse und Sichtweisen abgewogen werden müssen.

Das Methodenspektrum dieser Arbeit umfasst theoretische Modellierungen rationalen Verhaltens und Analysen von Politikfeldern, Konflikten, Instrumenten und Projekten unter der Perspektive der Umwelt- und politischen Ökonomie. Die Suche nach der Basis für kooperatives Verhalten und die Analyse von entsprechenden kooperativen bzw. partizipativen Modellen und Projekten einer partnerschaftlichen Lösung von Konflikten im Schnittstellenbereich des Naturschutzes und der Landwirtschaft sind der verbindende thematische Hintergrund dieser Arbeit.

Ergebnisse werden auf sehr verschiedenen Ebenen erarbeitet. Einerseits wird im Rahmen der Weiterentwicklung der theoretischen Grundlagen sozialer Interaktionen auf der Basis der erweiterten Kapitaltheorie und der Theorie der öffentlichen Güter (Kapitel 1) theorieorientierte Grundlagenarbeit geleistet. Durch die Einführung von Identitätskapital und Distinktionsnutzen in dem vom Becker et al. entwickelten Rahmen der erweiterten Kapitaltheorie gelingt die schlüssige Erklärung sozialer Interaktionen rationaler Individuen und damit eine Verbesserung der Erklärung der Theorie der öffentlichen Güter. Andererseits können auf praktischer Ebene Empfehlungen für Konfliktvermeidung und kooperative Problemlösung im Bereich der Agrarumwelt (Kapitel 2, 4, 5) und im Bereich der naturschutzfachlichen Eingriffsregelung (Kapitel 3) gegeben werden. 


\section{Ökonomische Beiträge zur Erschließung menschlichen Verhaltens für die Lösung von Naturschutzfragen}

\section{Einleitung}

Die Einheit aus sozialer und natürlicher Umwelt bildet die Lebensgrundlage und den Lebensraum des Menschen und steht dennoch nicht im Zentrum seiner Betrachtungen. Diese zunächst paradox erscheinende Erkenntnis ist, bei genauerem Hinsehen, ein allgemeines Prinzip. Das Alltägliche ist nicht der Rede wert, wird nicht beachtet und ist trotz grundlegender Bedeutung nicht Gegenstand unserer Aufmerksamkeit. Nur das Besondere, Auffällige bzw. Außergewöhnliche, das aus dem Alltag heraussticht, wird aktiv wahrgenommen ${ }^{1}$. Dies gilt nicht nur für den einzelnen Menschen, sondern auch für die Wissenschaften. Auch hier werden oftmals eigentlich augenfällige Grundlagen solange nicht wahrgenommen, wie sie sich nicht verändern oder die Theorieentwicklung behindern (Kuhn 1967 Kap. 3).

Im Rahmen der vorliegenden Arbeit wird die Bedeutung der Umwelt jeweils aus den Blickrichtungen der Ökonomie und des Naturschutzes betrachtet. Besonderes Augenmerk wird dabei auf die neueren Entwicklungen gelegt, die die Berücksichtigung neuer Umwelten im Rahmen von Theorien oder Konzepten dieser Wissenschaften herausgebildet haben.

Aus dem Bereich der Ökonomie wird einerseits der Blick auf die Umwelt- und politische Ökonomie fallen. Sie nähert sich Fragen des Schutzes der natürlichen Umwelt und entsprechender Steuerungsmöglichkeiten aus einer ökonomischen und politischen Sicht (Kapitel 2, 3, 4, 5). Die erweiterte Kapitaltheorie mit ihrem Sozial- und Humankapitalansatz stellt den Rahmen bereit, der es der Ökonomie erst erlaubt, auch jenseits der vertrauten Rahmenbedingungen des Markes Untersuchungen im gesellschaftlichen Bereich durchzuführen (Kapitel 1).

\footnotetext{
${ }^{1}$ Dieses Phänomen ist den besonderen Mechanismen der menschlichen Wahrnehmungs- und Informationsverarbeitung geschuldet. In der Sozialpsychologie wird dieses Phänomen als priming (Higgins et al. 1977), in der Soziologie und den Kommunikationswissenschaften als framing (Goffman 1974) beschrieben und erklärt, dass die menschliche Wahrnehmung durch Wahrnehmungsmodelle bzw. -rahmen gesteuert wird. Wahrgenommen werden die relevanten Aspekte, dies sind in der Regel die sich ändernden Parameter, und nicht die konstanten Rahmenbedingungen.
} 
Diese Arbeit steht unter einem Dilemma. Der Untersuchungsgegenstand des Naturschutzes ist ein Bereich der Praxis. Insofern ist dessen wissenschaftliche Betrachtung problematisch, da die von Max Weber geforderte Wertefreiheit der Wissenschaften kaum erreichbar scheint. Alle wissenschaftlichen Ergebnisse behaupten für sich unausweichlich normativen Charakter, entsprechend werden in einzelnen Kapiteln auch Politikempfehlungen gegeben, die zwar gut begründet sind, aber eben immer unter der eingeschlagenen Blickrichtung entwickelt wurden.

Auch einer Lösung des Dilemmas der Beschränkung auf modellhafte und methodische Betrachtungen kann diese Arbeit nicht näher kommen. Einerseits wird zwar die Erweiterung der ökonomischen Theorien um soziale und Identitätsaspekte vorgeführt und könnte somit als Blickfelderweiterung gesehen werden. Andererseits jedoch wird dies alles stets unter die ökonomische Theorie und Methodik gestellt. Um daher mit wenigen Worte auf die Probleme und externen Effekte des auch in dieser Arbeit genutzten „Imperialismus der Ökonomie“ im Sinne von Gary S. Becker hinzuweisen, ist in Kapital 6 anschließend die Parabel der kleinen Leute von Borka wiedergegeben. Diese Geschichte beschreibt, welche Verwirrungen und Gefahren die voreilige und unbedachte Übertragung von (wissenschaftlichen) Gedanken in den Alltag verursachen kann.

\section{Umwelt- und Naturschutz als Untersuchungsgegenstand}

Auch wenn gegenwärtig in Deutschland 100 Jahre staatlicher Naturschutz gefeiert (Froh/ Schmoll 2006) werden, ist der Umwelt- und Naturschutz als Politikbereich ein junges Feld sowohl staatlichen Handelns als auch wissenschaftlicher Beschäftigung.

Dabei ist der Umwelt- und Naturschutz in Deutschland als staatliche Querschnittsaufgabe definiert. Erst seit 1969 entwickelt sich in der Bundesrepublik der Umweltschutz als eigenständiger Politikbereich (Malunat 1994), 1970 wurde Prof. Grzimek zum ersten Bundesbeauftragten für Naturschutz ernannt, und mit dem Erlass des ersten Bundesnaturschutzgesetztes erfolgte 1976 die Ausweitung des Naturschutzes auch juristisch und instrumentell auf Gebiete, die außerhalb der klassischen Schutzgebiete liegen. Im Gefolge dieses Prozesses hat sich der Naturschutz von einer sich auf wenige Schutzgebiete konzentrierenden Nische zu einem flächendeckend zu berücksichtigenden Konzept entwickelt.

Aufgrund seiner Geschichte ist der Naturschutz in Deutschland stark ökologisch orientiert (Froh/ Schmoll 2006: 6, Plachter 1991: 9). Dass gesellschaftliche Aspekte wie Fragen der Finanzierung und Legitimierung von Naturschutz und der Akzeptanz und Durchsetzung der Naturschutzziele eine wachsende Bedeutung innerhalb des Naturschutzes erlangen, hat mehrere Gründe: 
Im Gefolge des Prozesses der Etablierung des Naturschutzes als Politikfeld und querschnittsorientierte Staatsaufgabe wird es für den Naturschutz nötig, sich stärker mit gesellschaftlichen, politischen und ökonomischen Aspekten zu befassen.

Zweitens hat das Konzept der Nachhaltigkeit, das weltweit politisch mit dem Brundtlandreport 1987 und der sog. Rio-Deklaration von 1992 zur zentralen gesellschaftlichen Leitlinie geworden ist, die Dreiseitigkeit aus ökologischen, ökonomischen und sozialen Gesichtspunkten in den Mittelpunkt des allgemeinen Handelns gestellt.

Und drittens trägt die fortschreitende Entwicklung des wissenschaftlichen Naturschutzes und die wachsende Wahrnehmung der Bedeutung des Naturschutzes dazu bei, dass sich zunehmend mehr Ansätze aus den Gesellschaftswissenschaften mit Aspekten von Naturschutz beschäftigen und sich sogar entsprechende Disziplinen (Umweltökonomie, Umweltsoziologie, Umweltpsychologie) herausgebildet haben. Die ursprünglich ökologische Basis des wissenschaftlichen Naturschutzes wird so um sozial- und gesellschaftswissenschaftliche Ansätze erweitert.

\section{Untersuchungskonzept}

Um die Ziele des Naturschutzes zu verwirklichen, ist eine Einflussnahme auf das Verhalten der Menschen nötig. Prinzipiell sind dazu verschiedene Kategorien von Instrumenten geeignet, sie können z. B. in Ordnungsrecht (z. B. Ge- und Verbote), Planung (Zentraler Ausgleich von Nutzungskonflikten) und überzeugungsorientierte Instrumente (Umweltbildung und -information, Kooperation und Beteiligung) oder in fiskalische (Öffentliche Ausgaben, Förderprogramme, Umweltabgaben) versus nicht-fiskalische (Kooperation, Planung, Auflagen) Instrumente unterteilt werden (siehe z. B. Hobbensiefken 1991, Abb. 28 oder Wicke 1993, Abb. 29).

Ohne auf die Probleme der Zielfindung im Naturschutz ausführlicher einzugehen (siehe z. B. Kapitel 3.1. und 3.2.), steht in dieser Arbeit die Weiterentwicklung des ökonomischen Verhaltensmodells und die Entwicklung und Bewertung von konkreten Steuerungsinstrumenten im Mittelpunkt.

Die Grundlage eines Instrumentes bildet eine Theorie darüber, wie dieses Instrument auf das Verhalten von Menschen einwirkt. Dazu wird ein Verhaltensmodell benötigt, das die Beobachtungen des Verhaltens verallgemeinert, ordnet und schematisiert. 


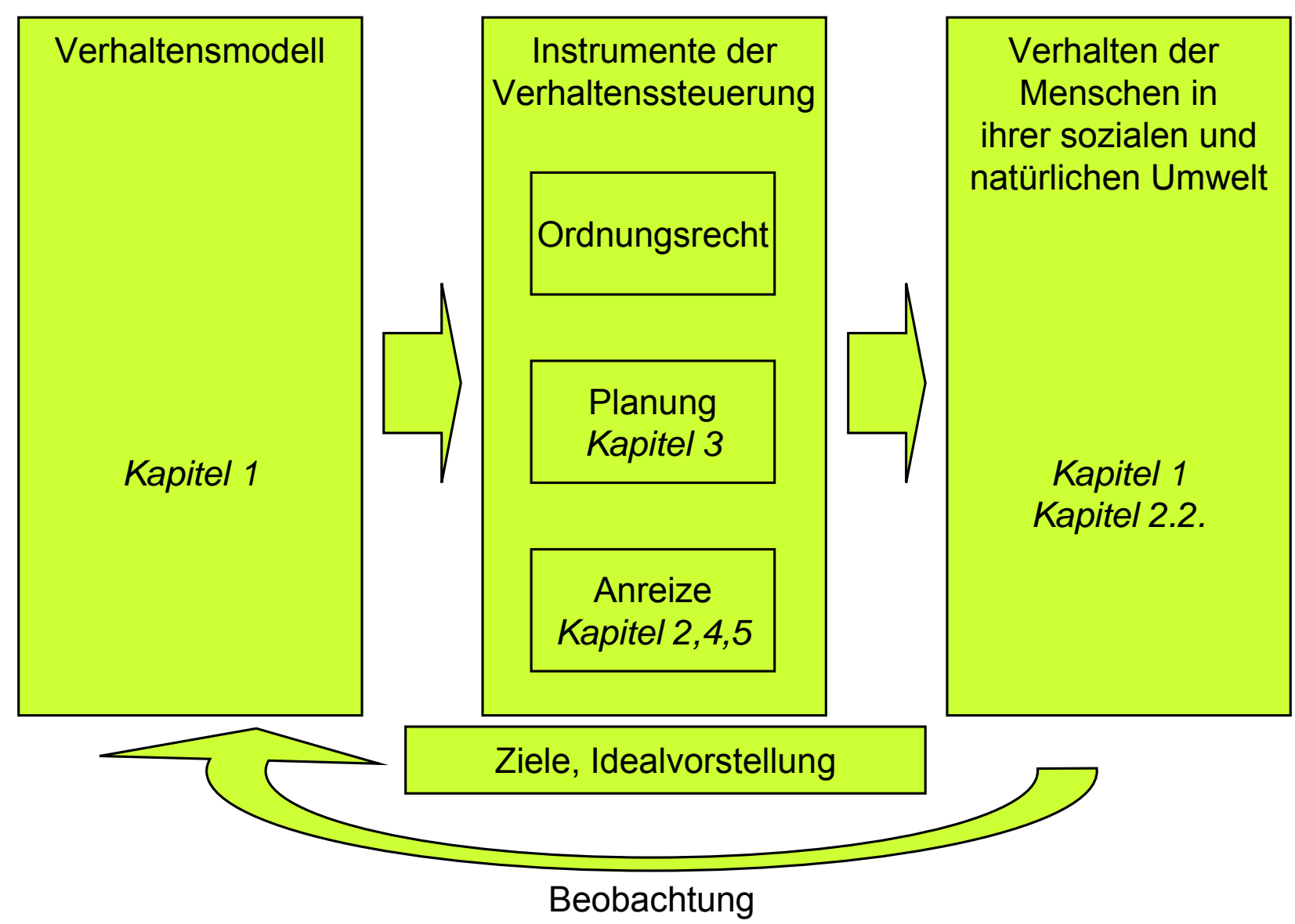

Abbildung 1: Im Rahmen der vorliegenden Arbeit werden Aspekte der Entwicklung eines Verhaltensmodells und darauf aufbauender verhaltenssteuernder Instrumente betrachtet. Die Beispiele stammen dabei aus dem Bereich des Umwelt- und Naturschutzes.

Kapitel 1 entwickelt daher, um menschliches Verhalten im Rahmen ökonomischer Theorien und Konzepte handhabbar zu machen, das Modell der rationalen Rekonstruktion sozialer Interaktionen weiter. Die interdisziplinäre Analyse soziologischer Austauschtheorien (Kap. 1.6.), psychologischer Identitätskonzepte (Kap. 1.7.1.), der erweiterten Kapitaltheorie von G.S. Becker et al. (Kap. 1.4.) und des Sozialkapitalkonzeptes (Kap. 1.5.) liefert Beiträge, die, ergänzt um die Konzepte Distinktionsnutzen und Identitätskapital (Kap. 1.7. und 1.8.), eine rationale ökonomische Rekonstruktion sozialer Interaktionen ermöglichen und damit eine Basis zur ökonomischen Erklärung sozialer Phänomene bereitstellen. In der Postmoderne ist Identität ein fragiles zu konstruierendes Konzept eines Individuums, um dessen Aufrechterhaltung sich das Individuum bemühen muss, es muss - ökonomisch gesprochen - in Aufrechterhaltung bzw. Pflege seiner Identität investieren und dazu Ressourcen aufwenden. Der Begriff Distinktionsnutzen wird eingeführt und verwendet, um den Nutzen, der dem Individuum bei jeder sozialen Interaktion entsteht, zur Identitätsstabilisierung beiträgt und als zentraler Anreiz für soziale Interaktionen dient (Kap. 1.7.2.1.), zu beschreiben. Um die Ressourcenaufwendungen mit anderen Aktivitäten eines Individuums in Beziehung setzen zu 
können, wird die erweiterten Kapitaltheorie von G. S. Becker et al. benutzt und um das Konzept des Identitätskapitals erweitert. Identitätskapital stellt dabei den individuellen Bestand an Merkmalen und Eigenschaften, die zur eigenen Identität gehören und in Interaktionen erworben wurden, dar (Kap. 1.7.2.2.) und modelliert diese gemäß der Kapitaltheorie als Kapitalbestand, der den anderen Kapitalarten (Geld-, Sach- und Humankapital) zu Seite gestellt wird. Identitätskapital fügt sich somit wie Human-, Sozial- und persönliches Kapital in die ökonomische Theorie ein.

Dieses Konzept ermöglicht die Interpretation vielfältiger Phänomene der Aufmerksamkeit und Erlangung sozialer Anerkennung (Kap. 1.9.). Es leistet z. B. einen Beitrag zum Verständnis der Ökonomie öffentlicher Güter, indem (1) die Entstehung des nötigen sozialen Umfeldes rational ökonomisch fundiert wird (Kap. 1.8.1.) und (2) der Distinktionsnutzen in Verbindung mit sozialer Kontrolle individuelle Beiträge zu öffentlichen Gütern rational macht (Kap. 1.8.2.).

Dieses Modell liefert einen Beitrag zur ökonomischen Interpretation menschlichen Verhaltens. Da es die individuellen psychologischen und sozialen Gegebenheiten besser berücksichtigt als einfache ökonomische Modelle (z. B. das des homo oeconomicus), ist dieses Modell auch besser geeignet, Probleme des Naturschutzes - und somit ökonomisch gesprochen der Bereitstellung bzw. Aufrechterhaltung öffentlicher Güter - und deren soziale Einbettung zu erfassen und verstehen zu helfen.

Der Naturschutz verfolgt gegenwärtig, in Abkehr von seiner ursprünglichen, auf Abgrenzung gegenüber den anderen Sektoren und Steuerung durch ordnungsrechtliche Regelungen setzende Strategie, eine Fokussierung auf anreizorientierte und partnerschaftliche Instrumente (siehe z. B. Kapitel 2.1.). Instrumente der überzeugungsorientierten Verhaltensbeeinflussung werden insbesondere deshalb präferiert, weil sie in dem Ruf stehen, eine nachhaltige Verhaltensänderung bewirken zu können und nicht unmittelbar von der Androhung von Strafe und Kontrolle, wie sie das Ordnungsrecht vorsieht, abängig zu sein. Daher werden in Kapitel 2 am Beispiel des Naturschutzes in der Agrarlandschaft die Schwierigkeiten und Chancen von partizipativen Lösungsansätzen für die Integration des Naturschutzes in die landwirtschaftliche Praxis demonstriert. Dazu müssen zunächst, um die Konfliktsituation zu verstehen, historische und psychologische Hintergründe des Konfliktes aufgezeigt werden (Kap. 2.1. und 2.2.). Anschließend wird die Vielschichtigkeit des Partizipationsproblems unter Nutzung von drei verschiedenen Perspektiven verdeutlicht (Kap 2.3.). Blickwinkel 1 stellt den überregional auf der Ebene der nationalen Politik und Verwaltung ablaufenden Prozess des Eindringens von Öffentlichkeit und Naturschutz in die Agrarpolitik dar. Blickwinkel 2 beleuchtet die 
Beteiligung der Landwirte an der Gestaltung der Regelungen und Förderprogramme. Zuletzt widmet sich Blickwinkel 3 der Partizipation der Bevölkerung bei der Zielfindung und Ausgestaltung der regionalen Landnutzung und des regionalen Naturschutzes. Als vielversprechende Lösung für viele der Partizipationsprobleme wird das Konzept „Regionale Partnerschaft für den Naturschutz in der Kulturlandschaft" vorgestellt (Kap. 2.4.).

In Kapitel 3 wird am Beispiel der naturschutzfachlichen Eingriffsregelung gezeigt, welches Potential das gut entwickelte Konzept der Zahlungsbereitschaftsanalysen bieten, um bisher wenig berücksichtigte, aber planungsrelevante Sachverhalte wie Landschaftsbild oder Identifikations- und Symbolfunktion bestimmter Naturgüter angemessen zu erfassen. Vor allem zur Erfassung der menschbezogenen Werte wie Landschaftsbild, Erholung, Heimat, Identifikations- und Symbolfunktion (Infobox 3.5.) sind Zahlungsbereitschaftsanalysen die adäquaten Methoden. Die Zahlungsbereitschaftsanalyse hat das Potential zur Ergänzung der Eingriffsregelung im Bereich der Bewertung der Beeinträchtigung des Landschaftsbildes oder der Erholungsfunktion und somit bei der Integration von Faktoren der sozialen Umwelt in eines der wichtigsten Naturschutzinstrumente.

Kapitel 4 und 5 bewerten Projekte, die auf Verhaltensmodellen basieren und verschiedene, schwerpunktmäßig anreizorientierte Instrumente zur Verbesserung des Naturschutzes in der Kulturlandschaft nutzen. Kapitel 4 konzentriert sich auf die Nutzungsmöglichkeiten von Agrarumweltprogrammen (AUP), die das wichtigste Instrument zur Verbesserung des Umwelt- und Naturschutzes in der Kulturlandschaft sind. Auf der Basis einer Analyse der AUP auf ihr ökologische Wirksamkeit, ökonomische Effizienz und ihre Akzeptanz bei den Landwirten wird vorgeschlagen, solche regionalen Partnerschaften zu schaffen bzw. zu fördern, deren Aufgabe das kooperative Management der Agrarumwelt ist. Diese Analyse wird gestützt durch Ergebnisse des „Bördeprojektes“ im Landkreis Wolfenbüttel (Niedersachsen, Deutschland), in dessen Rahmen mittels eines kooperativen regionalen Vorgehens die Agrarumweltmaßnahme „Blühstreifen“ für intensiv genutzte Agrarregionen entwickelt wurde. Nach der Darstellung der wichtigsten Erfolgsfaktoren des Projekts wird auf den die Ergebnisse zusammenfassenden Gesichtspunkt aufmerksam gemacht, dass lokale Partnerschaften in der Lage sind, das Thema Naturschutz auf die lokale Agenda zu bringen, lokal angepasste AUP zu entwickeln und eine Naturschutzberatung für die Landwirtschaft bereit zu halten und so die Aspekte Ökologie, Ökonomie und Akzeptanz auf regionaler Ebene zu vereinen.

Kapitel 5 schließlich untersucht die aufgrund der neuen, Beteiligung einfordernden europäischen Regelungen zur ländlichen Entwicklung verschiedene Beteiligungsmodelle der Programmsteuerung für Stakeholder des ländlichen Raums. Über den Vergleich zweier Projekte 
wird die Wirksamkeit verschiedener Ansätze zur Implementation der Stakeholderbeteiligung analysiert. Die Ansätze unterscheiden sich durch ihre unterschiedlichen Beteiligungsebenen und -strategien. Im niedersächsischen Projekt (wiederum das Bördeprojekt Wolfenbüttel) erfolgte die Beteiligung auf lokaler Ebene im Rahmen einer regionalen Partnerschaft, die lokal angepasste Agrarumweltmaßnahmen entwickelt hatte. Diese wurden nach erfolgreichem Test vor Ort in das Niedersächsische Agrarumweltprogramm übernommen. Im Projekt in Sachsen-Anhalt hingegen wurden die Stakeholder auf der Ebene der landesweiten Programmplanung integriert, indem mit Unterstützung eines mathematischen Modells verschiedene Varianten der Budgetverteilung auf einzelne Fördermaßnahmen gemeinsam durchgespielt und bewertet wurden. Beide Projekte zielten auf die Verbesserung der Akzeptanz der Agrarumweltprogramme. Es zeigt sich, dass deren auf verschiedenen Ebenen ansetzenden Instrumente zueinander komplementär sind und schon jedes für sich den Partizipations- und Akzeptanzgrad verbessern.

Kapitel 6 und das Fazit diskutieren die Ergebnisse mit Blick auf die Übertragung in ein gesellschaftliches Umfeld und deren Bedeutung für die Praxis.

\section{Literatur}

Froh H-W, Schmoll, F 2006: Amtlicher Naturschutz - Von der Errichtung der ,Staatlichen Stelle für Naturdenkmalpflege“ bis zur ökologischen Wende“ in den 1970er-Jahren. Natur und Landschaft Jg.81 Heft 1, S.2-7.

Goffman, E 1974: Frame Analysis: An Essay on the Organisation of Experimence. New York u. a.: Harper \& Row.

Hampicke, U 1992: Ökologische Ökonomie. Opladen: Westdeutscher Verlag.

Higgins, E T, Rholes, W S, Jones, C R 1977: Category accessibility and impression formation. Journal of Experimental Social Psychology 13: 141-154.

Hobbensiefken, G 1991: Ökologieorientierte Volkswirtschaftslehre, Oldenbourg Verlag, 2. Auflage München Wien.

Kuhn, T S 1967: Die Struktur wissenschaftlicher Revolutionen. Frankfurt am Main: Suhrkamp. (Englisch 1962).

Malunat, B M 1994: Die Umweltpolitik der Bundesrepublik Deutschland. Aus Politik und Zeitgeschichte B49/94, Beilage zur Wochenzeitung 'Das Parlament' vom 9.12.1994.

Plachter 1991: Naturschutz. Stuttgart: Gustav Fischer.

Wicke, L 1993: Umweltökonomie eine praxisorientierte Einführung, Verlag Franz Vahlen, 4. Auflage München. 


\section{Kapitel 1}

\section{Bausteine einer rationalen ökonomischen Rekonstruktion sozialer Interaktionen ${ }^{1}$}

\section{Warum auch ökonomische Modellindividuen sozial interagieren und öffentliche Güter erstellen}

\subsection{Einleitung}

Die Ökonomie will das Verhalten von Menschen anhand individualistischer, nutzenrationaler Modelle und Konzepte erklären. Dabei konzentrieren sich z. B. das Modell des homo oeconomicus und die eng mit ihm verknüpfte Kapitaltheorie darauf, individuelle Präferenzen und Nutzenerwägungen und die individuellen Güterbestände in Beziehung zu der zu analysierenden Wahlsituation eines Individuums zu setzen.

Schon früh trat dabei das Problem auf, dass bestimmte Situationen, oftmals soziale oder durch Kooperation geprägte Interaktionen, nicht schlüssig erklär- und voraussagbar waren. Im vorliegenden Artikel werden daher, ausgehend von den Theorien der sog. Allmende-, Umwelt- und öffentlichen Güter, die Probleme, die sich aus der Struktur der ökonomischen Denktradition ergeben, skizziert. Als zentrale Fragestellung wird dabei die individuelle Motivation zu sozialer Interaktion als kleinste Einheit des sozialen Gefüges thematisiert. Verschiedene Konzepte, die Beiträge in diesem Bereich der ökonomischen Theorie leisten, wie beispielsweise die Weiterentwicklung des ökonomischen Modellindividuums im Rahmen des Human- und Sozialkapitalansatzes (erweiterte Kapitaltheorie von G. S. Becker), Colemans Erweitung des Rational-Choice-Ansatzes und die Austauschtheorien der Nachbardisziplin Soziologie werden vorgestellt und als Grundlage für die Motivation zu sozialer Interaktion diskutiert. Ein Blick auf psychologische Konzepte der Identität gibt dann Hinweise für die in der Spät- und Postmoderne wachsende Bedeutung von individueller Identitätsentwicklung bzw. -erhaltung. Der gesellschaftliche Wandel erfordert es in wachsendem Maße, dass Identitätsentwicklung ein wichtiges Thema für Individuen wird und das - ökonomisch

\footnotetext{
${ }^{1}$ Manuskript (unveröff.): Jan Freese.
} 
gesprochen - erhebliche Ressourcen binden kann.

Hieraus werden mit Hilfe der Einführung eines Distiktionsnutzens eine funktionale Rekonstruktion und die Erweiterung der erweiterten Kapitaltheorie von Becker um das Konzept des Identitätskapitals als Modell zur ökonomischen Fundierung und Erklärung sozialer Interaktionen vorgeschlagen. Dieses Konzept wird schließlich für die Anwendung im Bereich der öffentlichen Güter genutzt.

Die Stärke des vorzustellenden Konzeptes liegt in seiner plausiblen Rekonstruktion individuell-rationalen Verhaltens in Bezug auf soziale Interaktionen und seiner moderaten Erweiterung der Beckerschen Kapitaltheorie, mit der eine theoriekonforme Fundierung sozialer Interaktionen erreicht und ein Konzept zur Überbrückung des (ökonomischen) Theoriebruchs zwischen individuellem Nutzenkalkül und Existenz des Sozialen entworfen wird.

\subsection{Allmende-, Umwelt- und öffentliche Güter}

Das Problem der öffentlichen Güter und der Übernutzung von Allmenden wird in der ökonomischen Literatur nicht erst seit Hardins „Tragedy of the Commons“ (1968) kontrovers diskutiert [zur Entwicklung der Theorie siehe z. B. Cornes/ Sandler (1999: 3 - 12) oder den Übersichtsartikel zur Diskussion im Entwicklungszusammenhang bei Ostrom et al. (1999)]. Diesen Gütern ist gemeinsam, dass man Individuen nicht von der Nutzung des Gutes ausschließt oder ausschließen kann (siehe auch Tab. 1). Allmendegüter sind einerseits viele Umweltressourcen wie Weiden oder Wald. Sie werden von einer Nutzergemeinschaft gemeinsam genutzt. Dabei haben die einzelnen Mitglieder zwar Nutzungs-, aber keine Besitzrechte. Andererseits können öffentliche Güter in einem Gemeinwesen staatlich bereitgestellte Güter wie z. B. Wege und Straßen oder ein Gemeindehaus sein. Die Nutzung von Allmendegütern ist konkurrierend, d.h. die Nutzung der Allmende durch ein Mitglied der Gemeinschaft verringert die Nutzungsmöglichkeiten der anderen Mitglieder (Gardner et al. 1990). $\mathrm{Zu}$ den öffentlichen Gütern, bei denen in der Reinform eine konkurrierende Nutzung nicht besteht, zählen aus dem Bereich der Umweltressourcen im üblichen Verständnis saubere Luft und Wasser, die Erholungsfunktion und Schönheit der Natur und aus dem Bereich der staatlich oder gesellschaftlich bereitgestellten Güter z. B. Rechtssicherheit (Justizsystem), innere Sicherheit (Polizei), äußere Sicherheit (Armee), Gesundheitsversorgung, Marktwirtschaft oder Küsten- und Hochwasserschutz ${ }^{2}$. Eine andere Gruppe von öffentlichen Gütern stellen Algorithmen, Computerprogramme, Mathematik, Sprachen, Theorien, Musik, Ge-

\footnotetext{
${ }^{2}$ Zur Definition von Umweltgütern und staatlich bereitgestellten öffentlichen Güter siehe z. B. Marggraf/ Streb (1997: 42).
} 
schichten oder Internetseiten dar, hierbei handelt es sich um Güter, die sich um die Bereitstellung von Informationen und Wissen drehen. Die Nicht-Konkurrenz der Nutzung bei reinen öffentlichen Gütern lässt sich z. B. an einer Landschaft verdeutlichen. Die Betrachtung durch eine Person verringert nicht die Betrachtbarkeit für andere Individuen. Solange Umweltgüter nicht knapp, sondern im Gegenteil in einem erheblichen Überschuss vorhanden sind, gilt dies auch für sauberes Wasser, Atemluft oder die Erholungsfunktion einer Landschaft.

Öffentliche Güter sind somit keineswegs selten ${ }^{3}$ und stellen einen wichtigen Untersuchungsgegenstand der Ökonomie dar.

Tab 1.1.: Eigenschaften der Güterklassen

\begin{tabular}{|l|c|c|}
\cline { 2 - 3 } \multicolumn{1}{c|}{} & \multicolumn{2}{c|}{ Individuen sind von der Nutzung... } \\
\cline { 2 - 3 } \multicolumn{1}{c|}{ Nutzung ist konkurrierend } & Privates Gut & $\begin{array}{c}\text { Allmendegut (staatliche und } \\
\text { Umweltgüter) }\end{array}$ \\
\hline Nutzung ist nicht konkurrierend & Clubgut & $\begin{array}{c}\text { öffentliches Gut (staatliche } \\
\text { und Umweltgüter) }\end{array}$ \\
\hline
\end{tabular}

Es gibt viele öffentliche Güter, die von einer finanziellen Beteiligung (z. B. Strassen, Infrastruktur) abhängen und solche, die kooperatives Verhalten benötigen (z. B. soziale Marktwirtschaft, gesellschaftliche Normen). Ein weiterer wichtiger Aspekt bei öffentlichen Gütern sind die Details der Bereitstellbarkeit, die von der Eigenart des öffentlichen Gutes abhängen (Sandler 2001). Die Bereitstellung oder Aufrechterhaltung eines öffentlichen Gutes kann gleichermaßen vom Beitrag aller Individuen abhängen. Im Fall der Luftreinhaltung oder der Kontrolle der globalen Erwärmung sind Beiträge aller nötig und führen in ihrer Summation zur Guterstellung (,summative supply technology“). Hiervon unterscheidet Sandler öffentliche Güter, deren Zustandekommen zwar Anstrengungen aller benötigt, aber letzten Endes lediglich vom schwächsten Glied abhängt (,weakest link supply technology“). Dies ist z. B. im Fall der Infektions- und Parasitenbekämpfung oft der Fall. So reicht eine unachtsame Person, die Trinkwasser mit Parasitenlarven oder infektiösen Keimen verunreinigt, oder ein Tierhalter, dessen Weidetiere gemeinschaftlich genutzte Weidegründe verseuchen, weil er seine Tiere nicht parasitenfrei hält, um die Herstellung dieses öffentlichen Gutes zu verhindern. In anderen Fällen ist bei der Bereitstellung nur der größte bzw. ein entscheidender Beitrag wichtig. Dies ist oft bei Technologien, Impfstofferfindungen oder der Medikamenten-

\footnotetext{
${ }^{3}$ Samuelson (1969: 109): "So I now think the useful terminology in this field would be pure private goods in which market mechanism works optimally, and possibly close approximations to them, versus the whole field of consumption-externalities or public goods." Und bei Weimann (1987: 272): ".. nicht das öffentliche Gut ist der polare Fall, der nur höchst selten auftritt und dessen Behandlung daher von allenfalls akademischem Interesse ist, sondern das private Gut befindet sich in der Rolle des Außenseiters."
} 
herstellung der Fall (,,best shot supply technology“). Letztlich gibt es auch noch die Möglichkeit, dass die verschiedenen individuellen Beiträge verschieden gewichtet summiert werden und zum öffentlichen Gut somit in unterschiedlichem Grad beitragen (,weighted sum supply technology“). Beispiele wären die Luftreinhaltung von spezifischen Schadstoffen (z. B. Schwefelverbindungen), die überwiegend von bestimmten Industrien oder in bestimmten Regionen entstehen (Sandler 2001). Eine andere Betrachtungsweise baut auf dem Konzept der zwei Schwellen auf. Die erste Schwelle ist die Mindestteilnehmerzahl oder Beitragssumme, die zur Erstellung oder Aufrechterhaltung nötig ist, und die zweite Schwelle ist die, ab der ein individueller Beitrag die Menge oder Qualität des öffentlichen Gutes nicht mehr weiter erhöht. Diese verschiedenen Einordnungen von öffentlichen Güter und Allmenden verdeutlichen die besondere Problematik, die Individuen bei der Frage der Beitragsleistung berücksichtigen müssen.

Da es im Folgenden um die Vorstellung eines sehr grundlegenden Mechanismus zur Erweiterung des ökonomischen Handlungsfeldes durch Nutzbarmachung von Aspekten der Individualität und des Individuum-Seins des ökonomischen Akteurs geht, wird nicht mehr systematisch zwischen Allmende, Umwelt-, staatlich und öffentliche bereitgestelltem Gut und den Bereitstellungseigenschaften (sensu Sandler) unterschieden, sondern einheitlich der Begriff 'öffentliches Gut' für Güter benutzt, bei denen einzelne Individuen einer Gemeinschaft nicht von der Nutzung ausgeschlossen werden bzw. werden können. Mit „Beitrag zur Bereitstellung und Aufrechterhaltung“ zu einem öffentlichen Gut wird ein kooperativer, freiwilliger Beitrag eines Individuums bezeichnet. Bei staatlich bereitgestellten öffentlichen Gütern und Umweltgütern können dies auch die Einhaltung von Nutzungsregeln und Normen sein, die Leistung eines eignen Beitrages, wie z. B. der Verzicht auf die Verschmutzung eines Umweltgutes, der Verzicht auf eine kostenfreie Nutzung oder die Reduzierung der Inanspruchnahme eines öffentlichen oder Umweltgutes auf ein Maß, das unterhalb der individuell optimalen Nutzung liegt.

\section{3. Öffentliche Güter in der ökonomischen Theorie}

Das zentrale, modellhafte Individuum der Ökonomie ist der homo oeconomicus, ein streng individualistischer rationaler Nutzen-Maximierer (Manstetten 2002, Kirchgässner 1991). Der homo oeconomicus setzt seine Ressourcen (Zeit, materielle und humane Ressourcen) zur Befriedigung seiner Bedürfnisse ein. Er tut das, was ihm bei der Bedürfnisbefriedigung nützt und unterlässt jenes, was ihm nicht nützt oder schadet. Aufgrund seiner Präferenzen kann ein Individuum entscheiden, welche der vielen Möglichkeiten (in der Ökonomie auch als Güterbündel bezeichnet) seine Bedürfnisse am besten befriedigen. Dabei strebt es aber die Maxi- 
mierung der Erträge der eingesetzten Ressourcen zur Bedürfnisbefriedigung an.

Aufgrund der Nicht-Ausschließbarkeit des Nutzungszugangs einzelner Individuen zu öffentlichen Gütern ist es in einer homo-oeconomicus-Ökonomie für ein Individuum irrational, sich an der Bereitstellung oder Aufrechterhaltung zu beteiligen, bzw. eine zurückhaltende Nutzung der öffentlichen Güter zu betreiben. Es würde stattdessen Nutzen maximierend von der sog. Trittbrettfahreroption Gebrauch machen, also die Nutzenstiftung des Gutes in Anspruch nehmen, aber keine Gegenleistung dafür erbringen. Der homo oeconomicus hat keinen Anreiz, schonend oder nachhaltig mit öffentlichen Gütern umzugehen (siehe z. B. Schlösser 1992: 109 oder Übersichten aus der experimentellen Ökonomie z. B. bei Davis/ Holt 1992, Ledyard 1995 oder genereller: Andreoni 1988, 1990, Andreoni/ Miller 2002).

\subsubsection{Von der Ein-Individuen- zur Mehr-Individuen-Ökonomie}

Generell betrachtet das Modell-Individuum der Neoklassik andere Individuen nicht, bzw. steht ihnen indifferent gegenüber. Es nimmt keinen Anteil am Nutzen anderer, weder durch Altruismus noch durch Missgunst. Der homo oeconomicus hat keinen Vorteil davon, dass andere Individuen einen Vorteil haben und er hat auch keinen Vorteil davon, wenn andere Individuen einen Nachteil haben. Die Haltung gegenüber seiner Mitwelt bezeichnet Rawls (1971: 168, siehe auch Kirchgässner 1991: 15f) als ,gegenseitige desinteressierte Vernünftigkeit“". Das Verhalten der anderen wird generell als Umwelt/ Umgebung und damit als Rahmen des eigenen Handelns, der Restriktionen setzt oder Möglichkeiten eröffnet, betrachtet.

Für Adam Smith, einer der Klassiker der Ökonomie, waren ,,andere Individuen“ eine eigene berücksichtigenswerte Kategorie. Er leitete seine „Theory of Moral Sentiments“ (1759), die 17 Jahre vor seinem ökonomischen Hauptwerk „An Inquiry into the Nature and the Causes of the Wealth of Nations“ (1776) erschien, wie folgt ein: „Mag man den Menschen für noch so egoistisch halten, es liegen doch offenbar gewisse Prinzipien in seiner Natur, die ihn dazu bestimmen, an dem Schicksal anderer Anteil zu nehmen, und die ihm selbst die Glückseligkeit dieser anderen zum Bedürfnis machen, obgleich er keinen anderen Vorteil daraus zieht, als das Vergnügen, Zeuge davon zu sein. Ein Prinzip dieser Art ist das Erbarmen oder das Mitleid, das Gefühl, das wir für das Elend anderer empfinden, sobald wir dieses entweder selbst sehen, oder sobald es uns so lebhaft geschildert wird, daß wir es nachfühlen können“ (Smith 1926: 1). Da die moderne Ökonomik in der ökonomischen Verhaltenstheorie aber auf die Geltung des Eigennutzaxioms setzt, umgeht sie das von Adam Smith aufgeworfene Problem, wie wohlwollendes und eigeninteressiertes Handeln theoretisch konsistent gleich- 
zeitig gedacht werden können (siehe hierzu z. B. Meyer-Faje/ Ulrich 1991) ${ }^{4}$.

Die Differenz zwischen dem empirischen Befund der Existenz von öffentlichen Gütern und ihrer Nicht-Integrierbarkeit in das ursprüngliche homo-oeconomicus-Modell ist somit seit langem eine Herausforderung für die ökonomische Theorie. Es gilt zu erklären, warum ein homo oeconomicus die Trittbrettfahreroption der kostenlosen uneingeschränkten Nutzung des öffentlichen Gutes in der Realität oft nicht wahrnimmt und öffentliche Güter nicht stets übernutzt werden. Die besondere Herausforderung für ökonomische Betrachtungen bei öffentlichen Gütern ist, dass der homo oeconomicus das öffentliche Gut gemeinsam mit anderen nutzt und diese Nutzung nicht der marktwirtschaftlichen, preis- und einkommensbestimmten Steuerung unterliegt. Aufgrund der Nicht-Ausschließbarkeit einzelner Individuen von der Nutzung des öffentlichen Gutes gibt es auch keine sonst den Besitz und damit die Verfügung und Nutzung regelnden Besitztitel (Property Rights).

\section{Ein-Individuum -Ökonomie}

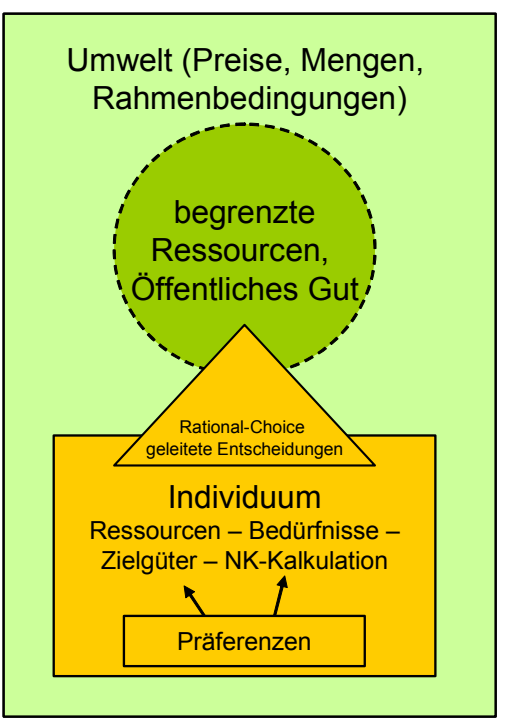

\section{Mehr-Individuen-Ökonomie}

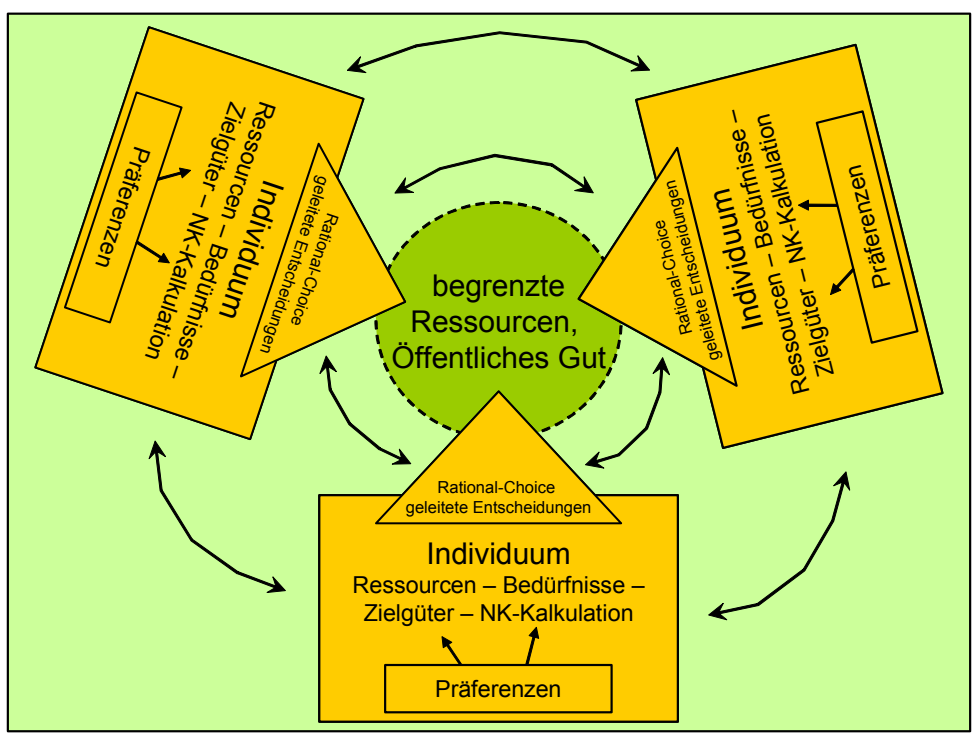

Abb. 1.1.: Im Unterschied zur Ein-Individuen-Ökonomie (einfaches homo-oeconomicus-Modell) folgen aus der Existenz anderer Individuen in einer Mehr-Individuen-Ökonomie komplexe Fragen der Interdependenz zwischen den Individuen und deren Verhalten.

In einem Modell, das mehr als ein Individuum zulässt, ergeben sich komplexe Fragen der Interdependenz zwischen diesen Individuen und deren Verhalten. Gibt es nur Interdependenzen mit den beobachteten Verhalten anderer Individuen? Oder wirkt sich allein schon die Existenz eines anderen Individuums auf die Entscheidung oder auf das Individuum als Ganzes aus? Wie weitgehend berücksichtigt das Individuum das mögliche Verhalten der anderen

\footnotetext{
${ }^{4}$ Einige Teildisziplinen wie die Neue Institutionenökonomie (Spieltheorie, Vertragstheorie, Principal-AgentTheorie) betrachten andere Individuen als besondere Objekte und erweitern die Annahmen des Basismodells z. B. um opportunistisches Verhalten oder Transaktionskosten (siehe auch 1.3.2.).
} 
Individuen bei seinen Entscheidungen? Wirken diese äußeren Einflüsse lediglich auf den engeren Entscheidungsmechanismus in einer Entscheidungssituation, also auf die NutzenKosten-Kalkulation oder gibt es Auswirkungen auf das Individuum als Ganzes, seine Zielgüter, sein Weltmodell und seine Ressourcen? So ergibt z.B. das Konzept einer Statusorientierung nur einen Sinn, wenn dem Individuum die Bewertung seiner Person durch andere Individuen relevant erscheint.

Wie geht der homo oeconomicus also mit dieser Situation um? Hinter allen Diskussionen steckt die Frage, wie kooperatives oder normgeleitetes Verhalten und somit das Gemeinwesen, bzw. das Soziale auf der Grundlage ökonomisch agierender Individuen entstehen kann, um z. B. öffentliche Güter zu schaffen und zu erhalten. Wie kann die ökonomische Theorie die Entstehung von kollektiven Regeln (Normen, Moral) und kooperativem Verhalten auf der Basis einer individuellen, rationalen Interessensverfolgung erklären?

\subsubsection{Lösungsstrategien}

Es geht um die Frage, ob individualistische ökonomische Modelle, die sich im Bereich von Marktgütern und Marktkoordination bewährt haben, auch die Interaktion und Kooperation mehrerer Individuen und somit die Schaffung einer Gemeinschaft, eines Gemeinwesens oder einer Gesellschaft erklären können. Kann eine ökonomische Theorie erklären, wie Normen und kollektive Regeln entstehen und warum sich Individuen den daraus resultierenden Restriktionen ihres individuellen Handelns unterwerfen? Dabei will die Ökonomie, anders als z. B. einzelne Richtungen der Soziologie, den methodologischen Individualismus und sozialen Subjektivismus, also die Annahme, individuelles Verhalten basiere auf den Interessen des Individuums, nicht aufgeben.

Ökonomische Modelle werden, um diese Probleme zu umgehen, oft so konstruiert, dass das Soziale, die Gesellschaft und gesellschaftliche Normen nur als existierende Restriktionen für ökonomische Fragestellungen verwendet werden. Die Fragen zu Entstehung und Interdependenzen des Sozialen und dessen Kompatibilität mit der ökonomischen Theorie, also z. B. die Vereinbarkeit von sozialen Restriktionen mit dem methodologischen Individualismus, bleiben außer Acht. Da für die Ökonomie die Probleme des Sozialen im Bereich der öffentlichen Güter so offen zu Tage getreten sind, dass sie für eine befriedigende ökonomische Behandlung des Problems nicht ignoriert werden können, gibt es hier bereits eine Vielzahl von Lösungsansätzen. Diese lassen sich verschiedenen Gruppen zuordnen:

1. Es ist durchaus üblich und vielfach möglich, hinter vermeintlichen Dilemmasituationen, also dem unterschiedlichen Beitrag verschiedener Akteure zu einem (scheinba- 
ren) öffentlichen Gut, nur eine unzureichende Analyse der Nutzenstrukturen und -kalkulationen zu vermuten. Das Beispiel eines attraktiven Landschaftsbildes einer Region (oft als öffentliches Gut bewertet), zu deren Herstellung und Aufrechterhaltung überwiegend eine Tourismus- oder Landschaftsbehörde durch Pflegemaßnahmen, aktive Gestaltungen und gezielte Subventionierung bestimmter das Landschaftsbild erhaltender Landschaftsnutzungen beiträgt, lässt sich durchaus ohne Dilemma konstruieren. Insbesondere, wenn z. B. von der Erhebung einer Kurtaxe oder eines Eintrittsgeldes zur Durchsetzung der individuellen Kostenbeiträge nach Abwägungen oder Wirtschaftlichkeitsanalysen aufgrund zu hoher Transaktionskosten und anderer erhoffter Gewinne (z. B. durch eine Abgrenzung gegenüber Regionen mit entsprechenden Abgaben) abgesehen wurde. In diesem Fall ist das Nutzungsrecht klar festgelegt, und auf die Durchsetzung einer gleichverteilten Kostenbeteiligung wird aus kalkulierten Gründen verzichtet (siehe z. B. Gardner et al. 1990).

2. Wenn von einem regelmäßigen Marktversagen bei der Bereitstellung von öffentlichen Gütern ausgegangen wird, wird daraus oft die Annahme abgeleitet, die Bereitstellung öffentlicher Güter könnte nur staatlich, also institutionalisiert und somit jenseits der Marktlogik sichergestellt werden. Da der Staat die Kosten auf alle Individuen verteilen und die pflegliche Nutzung regeln oder kontrollieren kann, werden die individuellen Beiträge im nötigen Umfang z. B. durch eine Steuer oder Umlage und durch Vorschriften der Beitragsleistung sichergestellt (siehe z. B. Diskussion zur Pigou-Steuer zurückgehend auf Pigou 1920).

3. Im Bereich der Neuen Institutionenökonomie (Überblicke z. B. bei Voigt 2002, Richter/ Furubotn 2003) wird das ökonomische Verhaltensmodell um einige realitätsnähere Annahmen erweitert. Aufgrund von empirischen Befunden der experimentellen Ökonomie und der Verhaltens- und psychologischen Forschung wurde das ökonomische Modellindividuum lernpsychologisch fundiert (Cyert/ March 1963, Simon 1955), mit einer lediglich begrenzten Rationalität (Selten/ Klopstech 1984) und asymmetrische Informationsverteilungen ausgestattet oder das Auftreten von Transaktionskosten (Coase 1984), opportunem Verhalten und unvollständigen Verträgen berücksichtigt. In der evolutorischen Ökonomik rücken zeitliche Aspekte und Fragen von Unsicherheit und Unwissen in den Vordergrund und werden bei individuellen Entscheidungen relevant. Das „Sich-Einlassen“ eines Individuums auf soziale Interaktionen und die Unterwerfung unter kollektive Regeln wird meist mit der Verringerung von Transaktionskosten (siehe hierzu z. B. Coase 1937, 1960, Williamson 1975, 1985) begründet. 
Es findet ein Tausch von individueller Entscheidungsfreiheit gegen eine bessere Güterversorgung statt (z. B. North 2002, Ostrom et al. 1994). Das Spektrum der hierzu einschlägigen Theorien ist groß: Die Property-Rights-Theorie (North 1988, 1990, Demsetz 1967, Alchian/ Demsetz 1972, Furubotn/ Pejovich 1972, 1974) untersucht zum Beispiel, welche Rolle die Existenz von Verfügungsrechten über Güter und deren Nutzung für die Institutionenbildung und Verhaltenssteuerung haben. Die PrincipalAgent-Theorie (March/ Simon 1958, Jensen/ Meckling 1976, Arrow 1985), analysiert Besonderheiten vertraglich vereinbarten Verhaltens und das Verhalten in Hierarchien. Die Verfassungsökonomie (Buchanan/ Tullock, 1962, Lee/ McKenzie 1987, Buchanan 1991, Brennan/ Buchanan 1993) analysiert die Bestimmungsgründe und Prozesse, die die Entstehung, Ausgestaltung und Entwicklung der gesellschaftlichen Institutionen steuern.

In diesem Zusammenhang darf auch nicht die Bedeutung von Moral und anderem gruppenorientierten Verhalten als Verhaltenssteuerung vergessen werden, die z. B. im Bereich der Wirtschaftsethik thematisiert wird (z. B. Sen 1977, Sudgen 1982, Laffont 1975, Ulrich 2001, Breuer et al. 2003, Homann/ Pies 1994).

4. Schließlich werden am Individuumsmodell einzelne Modifikationen durchgeführt, die zu einem direkten Anreiz für das Individuum zu sozialem Verhalten oder sozialer Interaktion beitragen sollen. So wurde die Einführung einer Präferenz für Altruismus vorgeschlagen, eine generelle Präferenz für soziale Interaktionen und soziale Anerkennung, Freundschaft oder Liebe postuliert ${ }^{5}$. Die Existenz einer Präferenz für Altruismus bedeutet demnach, dass die individuelle Aufwendung von Ressourcen mit dem Ziel des Wohls anderer Personen bzw. des Gemeinwohls dennoch dem Individuum einen individuellen Nutzen einbringen. Bekannt ist z. B. das Modell des „warm glow“ (Andreoni 1995, Andreoni/ Miller 2002). Dabei wird postuliert, dass sich ein Individuum besser fühlt - und damit einen egoistischen Nutzen hat - wenn seine Handlung positive Externalitäten auslöst, es z. B. für wohltätige Zwecke spendet oder anders zur Entstehung eines öffentlichen Gutes beiträgt. Dies wird auch ,impure altruism' genannt und bezeichnet eine Handlung die sowohl altruistische als auch egoistische Elemente beinhaltet.

$\mathrm{Zu}$ den Modellmodifikationen zählt auch, Ziele des homo oeconomicus zu begrenzen, ihm also nicht ein unbegrenztes Optimierungsziel vorzugeben, sondern anzunehmen, dass er bei einer guten Güterversorgung gesättigt ist oder nicht nach dem besten, son-

\footnotetext{
${ }^{5}$ Präferenzen und ihre Entstehung werden durch die Ökonomie nicht hinterfragt, sondern nur in ihren Auswirkungen, den aus den Präferenzen abgeleiteten Handlungen untersucht (Becker 1996: 3f).
} 
dern nur nach einem akzeptablen Güterbündel sucht und entsprechend nicht ein öffentliches Gut weiter ausbeutet, nur weil es kostenlos nutzbar ist. Das ambitionierteste entsprechende Modell stellt der REMM - resourceful evaluating maximizing man (Meckling 1976), bzw. der RREEMM - restricted resourceful expecting evaluating maximizing man - (Lindenberg 1985, Esser 1993: $231 \mathrm{ff})$ dar.

Um die Stärke der Ökonomie, den methodologischen Individualismus mit seinem Selbstbestimmungsaxiom (Weimann 1987: 243 f) auf der Basis von individuellen Präferenzen und einer individuellen Nutzen- und Entscheidungsfunktion $\mathrm{zu}$ erhalten und überhaupt Fragen normgeleiteten Verhaltens behandeln zu können, wäre es sehr hilfreich, eine auf dem methodologischen Individualismus und somit auf dem ökonomischen Modell des homo oeconomicus aufbauende Erklärung für soziale Interaktionen zu besitzen.

Dieses Kapitel entwickelt im Folgenden auf der Basis des individualistischen neoklassischen Individuumsmodells, der erweiterten Kapitaltheorie G. S. Beckers und unter Berücksichtigung konstitutiver Notwendigkeiten des Individuums ein Modell der Entstehung sozialer Interaktionen zwischen rationalen, nutzenmaximierenden (ökonomischen) Individuen, um auf diesem Weg einen Beitrag zur Erklärung der Existenz sozialer Interaktionen und des Sozialen und somit letzten Endes auch zur Erklärung der Entstehung und Erhaltung öffentlicher Güter zu leisten. Dazu erfolgt nach einer Darstellung der Grundlagen aus dem Bereich der um Human- und Sozialkapital erweiterten Kapitaltheorie und unter Nutzung von Konzepten der postmodernen Identität einerseits eine funktionale ökonomische Rekonstruktion einer direkten sozialen Interaktion zwischen zwei Individuen und andererseits die Übertragung dieses Konzeptes in den Rahmen der Kapitaltheorie.

\subsection{Die erweiterte Kapitaltheorie: Human- und Sozialkapital}

Kern der Kapitaltheorie ist, aus den Kapital- und Güterbeständen eines Individuums sein Verhalten in ökonomischen Wahlsituationen abzuleiten. Die erweiterte Kapitaltheorie hat dabei das ökonomische Konzept des individuellen Verhaltens in dreierlei Weise erweitert. Zunächst wurde klar gestellt, dass Präferenzen nicht direkt auf Güter abzielen, sondern allgemeinerer menschliche Bedürfnisse reflektieren, wie Nahrung, Wasser, Schutz, Wärme, sozialen Kontakt, etc. Diese nun auch Meta-Präferenzen (ultimate z, Haushaltsgüter oder commodities) genannten Präferenzen sind primäres Ziel menschlichen Verhaltens und werden vom Individuum produziert, indem Marktgüter konsumiert und Zeit für Nichtmarktgüter 
aufgewendet wird ${ }^{6}$. Anders als im herkömmlichen Modell werden Unterschiede in der Konsumstruktur von Individuen bei gleichen Einkommen mit unterschiedlichen Präferenzen erklärt. Hier geht die erweiterte Kapitaltheorie ebenfalls einen anderen Weg. Im Beckerschen Ansatz wird von identischen und stabilen Präferenzen gegenüber den verschiedenen Haushaltsgütern ausgegangen. Sämtliche Unterschiede in der Konsumentscheidung von Menschen werden auf Unterschiede in deren Gesamteinkommen und deren Produktionsmöglichkeiten für die Haushaltsgüter zurückgeführt. Die Annahme der Stabilität und der Identität bezieht sich auf die erweiterten Präferenzen, d. h. auf die Präferenzen, die u. a. Entscheidungen der Vergangenheit und die Einflüsse Dritter als Argumente der Nutzenfunktion einer Person berücksichtigen. Letzten Endes wird in der erweiterten Kapitaltheorie auch der Faktor der Zeit aufgewertet, indem vergangene Ereignisse und Erfahrungen ebenfalls als verhaltensrelevante Kapitalstöcke beschrieben werden und so die Vergangenheit die gegenwärtigen Entscheidungen mitbeeinflusst ${ }^{7}$.

Zunächst sollen die verschiedenen neu eingeführten individuellen Kapitalstöcke der erweiterten Kapitaltheorie beschrieben werden. Gary S. Becker hat, aufbauend auf den Arbeiten von T. W. Schultz durch die Einführung des Konzepts des Humankapitals die Erklärungswirkung der Ökonomie erheblich ausgeweitet (Becker 1993: 392, Habisch 1998: 32). Scheinbar nicht rational handelnde, weil nicht ihren offensichtlichen ökonomischen Vorteil nutzende Individuen brachten die neoklassische Ökonomie in Erklärungsschwierigkeiten. Schultz, der ursprünglich als Agrarökonom Entwicklungsrückstände der armen Bevölkerung von Entwicklungsländern untersuchte und sich hierbei mit der Frage befassen musste, warum breite Bevölkerungsschichten die Entwicklungsperspektiven der modernen Technik und der grünen Revolution nicht einsetzten, wies darauf hin, dass die ökonomische Wissenschaft zu sehr auf

\footnotetext{
${ }^{6}$ Um Widersprüche des ökonomischen Verhaltensmodells zu verarbeiten, differenzieren Stigler und Becker Bedürfnisse in (letzten Endes) menschliche Bedürfnisse (ultimative z, Meta-Präferenzen, Haushaltsgüter) und instrumentelle Güter (Zielgüter), die auf diese Bedürfnisse hin ausgerichtet sind. Später hat Becker (1996) das Modell Stigler/ Becker (1977) begrifflich klargestellt und dabei die Begriffe Meta- und Sub-Präferenzen eingeführt (siehe auch Abb. 1.2.). Metapräferenzen sind bei allen Menschen ähnlich, es sind die elementaren Bedürfnisse nach Ernährung, Fortpflanzung, Gesundheit und Sozialkontakten. Die Sub-Präferenzen werden kulturell und durch Erziehung geprägt. Dies ist aber insofern irrelevant, da die Ökonomie Präferenzen nur indirekt über Verhalten erfasst und sie aus methodologischen Gründen (Stigler/ Becker 1977, Becker 1993) als konstant annimmt.

${ }^{7}$ Das Forschungsprogramm „Humankapital“, das Schultz, Becker, Becker et al. und andere initiiert haben (Schultz 1973, Stigler/ Becker 1977, Becker 1962), hat Auswirkungen auf die Mikro- und Makroebene. Mikrotheoretisch erfolgte eine wesentliche Komplexitätssteigerung der Rational-Choice-Analyse durch Berücksichtigung intertemporaler Aspekte (Überblick z. B. in Becker 1993). Makrotheoretisch entsteht eine Verbreiterung der theoretischen Grundlagen demokratischer Wirtschafts- und Gesellschaftspolitik (Habisch 1998: 34). Aus diesem Grund ist insbesondere im Entwicklungszusammenhang das Thema Humankapital sehr wichtig geworden (siehe z. B. den Forschungsschwerpunkt Humankapital der Weltbank) und hat zu einer neuen Entwicklungstheorie geführt (Habisch 1998: 35). Die Notwendigkeit bestand hier darin, das Scheitern der Finanzspritzen z. B. des Internationalen Währungsfonds (IWF) und der Weltbank zu erklären. Diesen Teil der politologisch motivierten Human- und Sozialkapital-Diskussion nimmt Becker selbst jedoch explizit nicht zu Kenntnis, da er an Theoriebildungsfragen und nicht an Implikationen interessiert ist (Habisch 1998: 44).
} 
die Akkumulierung von Sachkapital fixiert sei (Schultz 1980: 640). Der Ausschluss zentraler menschlicher Lebensbereiche wie Ehe, Familie und Familienhaushalt aus den Wachstumsund Produktionsprozessen und deren alleinige Bewertung als Konsumsektor einer Volkswirtschaft führten zu erheblichen Erklärungsdefiziten und blinden Flecken der neoklassischen Theorie (Habisch 1998: 33).

Deshalb erweiterten Schultz, Becker und andere das ökonomische Handlungsmodell ${ }^{8}$. Dafür hielten sie bei abweichendem, zunächst nicht rational wirkendem Verhalten an der elementaren ökonomischen Logik der Rationalität fest und suchten nach objektiven und subjektiven Nebenbedingungen (Restriktionen), die das beobachtete Verhalten rational machten. Dazu stellten sie den klassischen Kapitalarten Arbeitskraft, Geld und Sachmittel das Humankapital als individuelles Wissen und individuelle Fähigkeiten zur Seite. Über das Humankapital können Komponenten wie Wissenserwerb, Ausbildung, Familien- und Bekanntschaftspflege in das ökonomische Rationalitätskonzept eingefügt werden. Personen, die nicht in die Maximierung ihrer Geld- und Sachmittel investieren, stecken ihre Ressourcen in die Maximierung ihres Humankapitals, investieren also z. B. in eine Ausbildung oder in die Familie (z. B. Becker/ Murphy 1996: 220). Dabei verwendet Becker den Begriff des Humankapitals als Überbegriff für die nichtmateriellen individuellen Ressourcen, unter die er das individuelle Wissen und die Fähigkeiten (Humankapital in engeren Sinne), vergangenen Konsum, persönliche Erfahrungen der Vergangenheit, Erziehung und Angewohnheiten (persönliches Kapital) sowie Beziehungen bzw. das individuelle Beziehungsnetzwerk (Sozialkapital) subsumiert (Becker 1996: 4).

Becker führt das persönliche Kapital $\left(\mathrm{P}_{\mathrm{t}}\right)$ ein, um eine Lösung für das Problem der nicht gleichartigen und größenähnlichen Reaktion auf zwei entgegengesetzte Preisänderungen bzw. die verschiedenartige Anpassungen der Menschen verschiedener Landesteile auf ein Opportunitätskosten änderndes Gesetz zu finden (z. B. Akerlof 1970, Tversky/ Kahneman 1974, Kahneman/ Tversky 1979, Kahneman/ Tversky 1984). Dieses auch als Problem der homogenen Restriktionen bezeichnete Modell geht darauf zurück, dass eine Verhaltensänderung durch das methodologisch bedingte Konstanthalten der Präferenzen allein auf der Änderung der Restriktionen beruhen kann . Um zu erklären, warum Menschen bei gleicher Güterausstattung und somit gleichen offensichtlichen individuellen Restriktionen auf eine einheitliche Änderung von externen Restriktionen (z. B. Preise oder Gesetze) verschieden reagieren, muss

\footnotetext{
${ }^{8}$ Im Folgenden wird ein zusammengesetztes Modell vorgestellt, das überwiegend auf Beckers zahlreichen Publikationen aus den Jahren 1962 bis 1996 beruht. Dabei ist zu beachten, dass Becker Ansichten sich verändert haben und er bestimmte Aussagen früherer Texte später verändert oder relativiert hat.

${ }^{9}$ Bei $\Delta V=f\left(P_{\text {const., }} \Delta \mathrm{R}\right)$ mit Verhaltensänderung $(\Delta V)$, konstanten Präferenzen $\left(\mathrm{P}_{\text {const. }}\right)$, Restriktionen $(\Delta \mathrm{R})$.
} 
er das persönliche und das Sozialkapital als individuelle interne Restriktionen einführen. Die sich im Zeitverlauf ändernden und individuell verschiedenen Einflussfaktoren der individuellen Vergangenheit und des individuellen sozialen Umfeldes können nur als Bestandsgrößen (Kapitalstöcke), als interne Restriktionen, verhaltensrelevant werden (Habisch 1998: 41). Individuelle Bestandsgrößen sind dabei Angewohnheiten, Sichtweisen und Habitualisierungen, die Becker „persönliches Kapital“ nennt. So kann er das scheinbar zeitinkonsistente Verhalten als unterschiedliche Habitualisierung des Diskontierungsverhaltens beschreiben. Im Lebensverlauf auftretende verschiedene Bestände persönlichen Kapitals beeinflussen das Verhalten bei ansonsten gleichartigen Entscheidungssituationen mit ähnlichen Nebenbedingungen und können so gegensätzliches Verhalten zwischen Personen und unterschiedliches Verhalten einer Person zu verschiedenen Zeitpunkten bei ansonsten ceteri paribusBedingungen erklären. Formal wird der Kapitalstock des persönlichen Kapitals P zum Zeitpunkt $t$ von Becker wie folgt angegeben:

$$
\mathbf{P}_{\mathrm{t}+1}=\mathrm{x}_{\mathrm{t}}+\left(1-\mathrm{d}_{\mathrm{p}}\right) * \mathbf{P}_{\mathrm{t}} \quad \text { (Gleichung 1.4.1) }
$$

mit $\mathrm{x}$ als Investition in persönliches Kapital und $\mathrm{d}_{\mathrm{p}}$ als konstante Abschreibungsrate (Becker 1996: 7).

Stabiler ist nach Becker der Einfluss sozialer Netzwerke auf das Verhalten. Zum sozialen Kapital sensu Becker (S) gehören Freunde, Kultur und Tradition, Milieu und Ideologie. Formal drückt er dies aus als:

$$
S_{t+1}^{i}=X^{i}+\left(1-d_{s}\right) * S_{t}^{i} \quad(\text { Gleichung 1.4.2) }
$$

mit der konstanten Abschreibungsrate $d_{s}$ und $X^{i}=\sum x^{j}$ als Effekt der Entscheidungen der $\mathrm{j}$ Mitglieder in i’s Netzwerk auf sein Sozialkapital (Becker 1996: 12).

Der Ansatz des sozialen Kapitals trägt der soziologischen Kritik Rechnung, dass Interaktionen nicht frei, sondern situativ gebunden sind und von verschieden Faktoren - nicht nur vom Preis - determiniert werden.

"Der einzelne entscheidet unter Abwägung von Kosten und Nutzen über seine Bildung, Berufsausbildung, medizinische Versorgung und andere Verbesserungen seiner Kenntnisse und seiner Gesundheit. Zu den Nutzen zählen kulturelle und andere nicht-pekuniäre Vorteile sowie die Verbesserung der Einkommens- und Berufssituation, während die Kosten vor allem vom entgangenen Wert der Zeit abhängt..." (Becker 1996 (1993): 29)

Somit ergibt sich für Beckers erweiterte Kapitaltheorie zum Zeitpunkt $t$ folgende Nutzenfunktion, die nicht nur von den konsumierten Gütern y, z abhängt, sondern auch vom Bestand an 
persönlichem (P) und Sozialkapital (S) abhängt:

$$
\mathrm{U}=\mathrm{U}\left(\mathrm{y}_{\mathrm{t}}, \mathrm{z}_{\mathrm{t}}, \mathbf{P}_{\mathrm{t}}, \mathrm{S}_{\mathrm{t}}\right) \quad \text { (Gleichung 1.4.3) }
$$

Becker hat ein Modell, das sog. Haushaltsproduktionsmodell, entwickelt, dass das Individuum (bzw. dessen Haushalt) vom volkswirtschaftlichen Konsumenten zum Produzenten weiterentwickelt. Das Individuum optimiert und akkumuliert nicht ausschließlich Sach- und Geldkapital, sondern kann dieses, zusammen mit anderen Ressourcen wie Zeit und seiner Arbeitskraft, in die Erstellung und Erhaltung von Humankapital (persönlichem und Sozialkapital) investieren.

In diesem ökonomischen Modell ist es - anders als in einfachen ökonomischen Modellen nicht mehr irrational, seine Zeit mit Freunden und Bekannten, der Pflege der eigenen Gesundheit oder in der Schule zu verbringen, anstatt die Zeit zu nutzen, um durch den Einsatz seiner Arbeitskraft Geld zu verdienen. Die Präferenzen bzw. Zielgüter des erweiterten homo oeconomicus sensu Becker führen dazu, dass nur ein Teil seiner Ressourcen (Zeit, Arbeitskraft, Kapitalstöcke) für Konsum (Sachkapitalaufbau) und Lohnarbeit (Geldkapitalaufbau) eingesetzt wird. Ein anderer Teil dieser Ressourcen wird in die Entwicklung des Humankapitals investiert. Erst mithilfe der Erweiterung der ökonomischen Kapitaltheorie um die Humankapitalkomponente wird erklärbar, warum individuelles Sach- und Geldkapital zugunsten der eigenen Ausbildung, der eigenen Gesundheit oder für eine Feier mit Freunden und damit in die Bildung von Humankapital investiert wird. Becker erkennt so dem ,privaten Sektor“ und dem Familienhaushalt eine zentrale Funktion insbesondere im sozialen und sozialisierenden Bereich zu (Habisch 1998: 35).

Im Zuge des Selbst-Managements passen Individuen ihren Lebensstil und ihr Beziehungsnetz ihren Präferenzen an, so dass ihr soziales Netz zur Maximierung ihres Nutzens beiträgt und gleichzeitig zu einer Verstärkung und Stabilisierung ihrer Präferenzen und daraus abgeleiteten Gewohnheiten führt ${ }^{10}$ (Habisch 1998: 42).

Becker lässt eine endogene Präferenzbildung ebenso wie Kultur, die Abhängigkeit der Präferenzen von vergangenen Erfahrungen, Pfadabhängigkeit und Intertemporalität zu, bleibt dabei aber strenger Anhänger des Rational-Choice-Ansatzes (Habisch 1998: 42f). Er plädiert dafür, Präferenzänderungen nicht leichtfertig als Erklärung heranzuziehen. Er modelliert Werbung z. B. so, dass deren Mechanismus nicht die Änderung von Präferenzen ist, sondern dass es vielmehr um die bessere Information des (potentiellen) Konsumenten über den

\footnotetext{
${ }^{10}$ Becker legt auf diesem Weg z. B. die Grundlage für die ökonomische Interpretation des Verhaltens eines Süchtigen und der individuellen Rationalität dieses Verhaltens (Stigler/ Becker 1977: 77, Becker 1996: 85, Becker/ Murphy 1988 aufbauend auf Ryder/ Heal 1973, Boyer 1978, 1983).
} 
Produktnutzen geht. Bei einer Verhaltensänderung infolge Werbung ändern sich nicht die Präferenzen, sondern die individuelle Abwägung, also die Bilanzierung des Nutzens und der Kosten infolge der zusätzlichen Informationen durch Werbung. Der Konsument erreicht durch den Kauf des beworbenen Zielgutes einen höheren Nutzen, der seinen Präferenzen besser entspricht (Stiegler/ Becker 1977, Becker/ Murphy 1993).

Pies beschreibt den normativen Beitrag von Beckers Forschungsprogramm als Wechsel der Blickrichtung ökonomischer Analyse weg von den Präferenzen hin zu den Restriktionen als „universale Erklärungsgrammatik sozialer Phänomene“ (Pies 1998: 26).

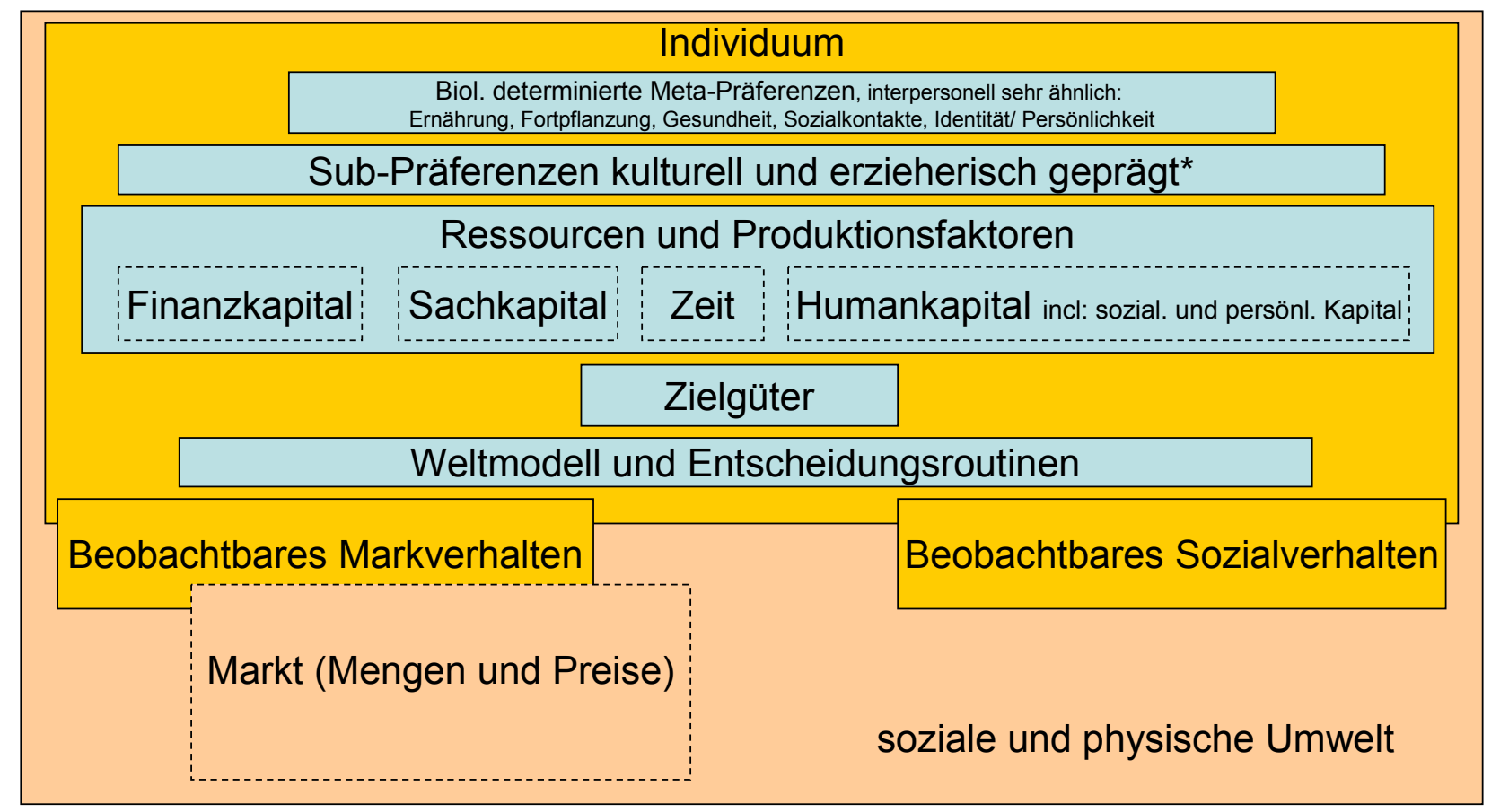

Abb. 1.2.: Modell des homo oeconomicus sensu Becker: Die Ziele eines Individuums drücken sich in Meta-, Subpräferenzen und schließlich Zielgütern aus. In der akuten Entscheidungssituation entscheiden dann Kosten-Nutzen-Abwägungen unter Berücksichtigung der Kapital-Bestandesgrößen (Geld-, Sach-, Humankapital) und dem Weltmodell über das Verhalten des Individuums. Die Befriedigung der Präferenzen, umgangssprachlich die Wünsche eines Individuums, erfolgt über eine entsprechende Anpassung der verschiedenen Kapitalbestände. Dabei ist auch die Umwandlung der Kapitalarten ineinander, also z. B. die Investition von Zeit und Geld in die eigene Bildung möglich.

* Aus ökonomischer Perspektive nicht beobachtbar, werden methodologisch als konstant und zeitstabil gesetzt. Änderungen im Verhalten sind auf Änderungen des Kapitalbestandes und der Produktionsfaktoren zurückgeführt: Bildung, Lernen, Moral, Erfahrungen der Vergangenheit (siehe auch Fußnote 7).

Um nachfolgend Begriffsverwirrungen zu vermeiden, sei auf eine Anomalie der Beckerschen Nomenklatur hingewiesen. Becker benutzt den Begriff Humankapital als Überbegriff für persönliches und Sozialkapital. In der übrigen Wissenschaft hat sich überwiegend die Benutzung des Begriffs Humankapital für die Fähigkeiten und Kenntnisse des Individuums etabliert, das gleichberechtigt neben dem Sozialen Kapital des Beziehungsnetzwerkes steht. Daher werden im Folgenden, wenn explizit nur die individuellen Kenntnisse, Fähigkeiten und 
Fertigkeiten gemeint sind, diese mit dem Begriff Humankapital benannt. Der Beckersche Überbegriff aller immateriellen Ressourcen wird bei Bedarf mit ,Humankapital im weiteren Sinn' (i. w. S.) bezeichnet.

\subsection{Das Konzept des Sozialkapitals von Coleman}

Der Begriff „Sozialkapital“ ist keine Erfindung von Becker und hat eine sehr heterogene Entstehungsgeschichte. Da das Konzept von verschiedenen Personen in unterschiedlichen Kontexten entwickelt wurde, unterscheiden sich Ziele und Konzepte z. T. erheblich (Haug 1997, Feldmann/ Assaf 1999). Bisher sind aus dem Sozialkapitalbegriff überwiegend qualitative Analysen hervorgegangen und Indikatoren abgeleitet worden. Eine formalisierte, deduktive und überprüfbare Theorie fehlt hingegen (Dieckmann 1993: 23) ${ }^{11}$. Weil Coleman den Begriff mit Blick auf den Rational-Choice-Ansatz modelliert hat und er eine entwickelte theoretische Grundlage vorlegen kann (Jans 2003), soll hier sein Konzept zur Ergänzung des Beckerschen Ansatzes kurz vorgestellt werden.

Coleman (1988, 1990) verfolgt mit seinem Sozialkapitalkonzept eine Erweiterung des Rational-Choice-Ansatzes. Ausgehend von rational handelnden, mit Zielen (Präferenzen) und Ressourcen ausgestatteten Individuen erweitert Coleman die Ressourcen des Individuums um Sozialkapital in Form von sozialen Strukturen, die, wie er sagt, wie anderes Kapital auch, produktiv sind, da sie Handlungen und Güterversorgungen ermöglichen, die ohne die sozialen Beziehungen nicht möglich wären (Coleman 1988: 98). Anders als andere Kapitalarten, auch anders als das einer Person eigene Humankapital (seine individuellen Fähigkeiten und sein Wissen, siehe oben, Becker 1962, Schultz 1961), haftet Sozialkapital senus Coleman nicht einer Person an, sondern existiert in der Beziehung zwischen Individuen. Sozialkapital besteht aus Verpflichtungen und Erwartungen gegenüber anderen Personen, Vertrauen in Beziehungen und Absprachen, Sozialstrukturen, Normen ${ }^{12}$ und sozialen und gesellschaftlichen Institutionen ${ }^{13}$ (Coleman 1988: $102 \mathrm{ff}$ ).

\footnotetext{
${ }^{11}$ Durlag (2002) kritisiert, dass auch zentrale Aufsätze zum Thema Sozialkapital unter vagen Konzepten, einer schwachen empirischen Basis, fehlender Angaben von Bedingungen der (Allgemein-) Gültigkeit und fehlende Informationen zur Plausibilität von Grundannahmen leiden.

${ }^{12}$ Normen sind gesellschaftlich vereinbartes Verhalten, das sozial beobachtet, kontrolliert und bei Fehlverhalten sanktioniert wird. Eine ökonomisch orientierte Definition liefert z. B. Pareto 1980. Anzumerken ist, dass dies zu individuellem Ausweichverhalten auf nicht beobachtbaren Bereichen führen kann: zu starke Normen können so auch kontraproduktiv für Kooperation sein. Das Paradebeispiel hierfür ist die Mafia. Hierbei wird auch deutlich, dass Normen externe Effekte auf Outsider haben und man daher vielleicht besser von der Zugehörigkeit zu einer individuell nützlichen normbefolgenden Gruppe sprechen sollte, anstatt generell die Existenz von Normen als individuell positiv zu bewerten (Jans 2003: 23, Diekmann 1993: 13).

${ }^{13}$ Die in der Regel positive Konnotation von Sozialkapital im gesellschaftlichen Kontext ist sicher prinzipiell richtig. Es darf aber nicht außer Acht gelassen werden, dass eine hohe Sozialkapitalausstattung auch negativ bewertbare Folgen wie Vetternwirtschaften, Seilschaften, Kartelle, Mafia, Monopole, etc. repräsentieren kann und sich infolge dessen Fragen von Abhängigkeiten, Macht und Herrschaft anschließen. Üblicherweise
} 
Coleman untersucht das Sozialkapital auch auf Aspekte öffentlicher Güter. Da ein Individuum mit der Inanspruchnahme einer sozialen Beziehung für die Befriedigung eines Bedürfnisses eine Verpflichtung für eine Gegenleistung eingeht, muss man davon ausgehen, dass ein Individuum es bevorzugt, seine Bedürfnisse ohne Nutzung des sozialen Umfeldes zu befriedigen. Somit würde ein Individuum es vorziehen, nicht sozial zu interagieren und somit auch keine sozialen Strukturen aufzubauen und zu unterhalten (Coleman 1988: 117, Diskussion zum Charakter von Sozialkapital als öffentliches Gut siehe auch z. B. Hermann-Pillath/Lies 2001). Da soziale Verpflichtungen nicht im juristischen Sinn abgesichert sind, stellt sich die Frage, warum Individuen sich auf „,soziale Verpflichtungen“ einlassen sollen, wenn man auch einen rechtsgültigen Vertrag machen könnte. Coleman schreibt, dass die Abwägung zum Einsatz von Vertrauen die Abwägung zwischen Kosten und Nutzen sei. Ob Vertrauensgewährung oder -verweigerung andere Individuen beeinflusst, ihnen Aktivitäten ermöglicht oder diese verhindert, wird nicht berücksichtigt (Coleman 1988: 117). Folglich ergibt sich nach Coleman ein Ungleichgewicht in der Investition eines Individuums in privates und soziales Kapital. Da ein Individuum den Nutzen seiner sozialen Aktivitäten nicht auf sich selbst beschränken kann, investiert es verhältnismäßig wenig in soziales Kapital, auch wenn es eine sehr wichtige Ressource für den Alltag und die Erreichung eines guten Lebensstandards darstellt. Coleman geht somit davon aus, dass Sozialkapital nicht im Fokus der Individuen steht und seine Bildung und Zerstörung Nebenprodukte individuellen Handelns sind (Coleman 1988: 118).

Coleman bringt auf diese Weise die Bedeutung sozialer Strukturen in den Rational-ChoiceAnsatz ein und verbindet die kontextbezogene Modellierung von Handlungen, wie sie in der Soziologie üblich ist, mit dem methodologischen Individualismus der Ökonomie.

\subsection{Soziologische Austauschtheorien}

Um das Ziel der besseren Repräsentanz sozialer Interaktionen und des Sozialen in ökonomischen Modellen nicht nur disziplinär zu betrachten, erscheint es angebracht, die soziologischen Austauschtheorien in die Betrachtung einzubeziehen.

Der Grundgedanke der Austauschtheorien besteht in der Annahme, Menschen würden soziale Beziehungen eingehen bzw. aufrecht erhalten, um dadurch eine 'Belohnung' zu erhalten. Eine interpersonale Beziehung kommt somit durch einen Austausch zwischen Personen zustande, wobei diese in unterschiedlichen Relationen verwickelt sein können. Man kooperiert mit

verschließt sich die Ökonomie entsprechenden (gesellschaftlichen) Wertungen nach Verteilung, Abhängigkeit und Macht und zieht sich auf ihre Effizienzüberlegungen zurück. Die Verwendung des Sozialkapitalbegriffs trägt aber das Problem entsprechender Wertungen und Diskussionen unweigerlich in die Ökonomie hinein. 
anderen, weil man dadurch seine Bedürfnisse besser befriedigen oder seine Ziele besser erreichen kann bzw. weil man eine intrinsische Belohnung etwa in Form eines 'guten Gefühls' erfährt, wenn man Schwächeren hilft.

Trotz der Nähe zur ökonomischen Theorie beschränkt sich Austausch im soziologischen Sinn nicht auf wirtschaftliche Interaktion, sondern es existieren viele mögliche Austauschformen: Man tauscht Ideen aus, Nachbarn helfen sich gegenseitig, Gefühle oder Ratschläge werden ausgetauscht. Dabei wird der Austausch, anders als in der Ökonomie, nicht als rein rational, sondern auch von emotionalem, religiösem, irrationalem oder normgesteuertem Verhalten beeinflusst angesehen.

Blau (1964) zufolge liegt das Hauptproblem einer Theorie sozialer Strukturen darin, die komplexen gesellschaftlichen Makrostrukturen aus den einfachen sozialen Prozessen herzuleiten, die von den täglichen Interaktionen zwischen Individuen und ihren interpersonalen Beziehungen durchdrungen sind. Institutionalisierte Werte und Normen setzen nur den Rahmen, innerhalb dessen die Individuen, ihren Interessen folgend, vielerlei freiwillige Beziehungen eingehen und so dem Austausch eine unvorhersagbare Eigendynamik geben können. Blau geht davon aus, dass es beim Austausch keine feste Reziprozitätsnorm gibt, die festlegt, welche Leistung zu welcher Gegenleistung verpflichtet und an die sich alle Gesellschaftsmitglieder in jeder Situation halten könnten. Vielmehr führt er die Reziprozität auf die Bedingungen des Austausches selbst zurück. Es sei notwendig, dass Personen aufgrund ihres Interesses an fortgesetzter Erhaltung von Gratifikationen bzw. Diensten, die sie nur innerhalb der sozialen Interaktion erhalten können, ihren Verpflichtungen gegenüber anderen Personen nachkommen. Dies fördert die Entwicklung möglichst freundschaftlicher vertrauensvoller Beziehungen. Somit wird infolge des kooperativen Verhaltens auch die Erstellung öffentlicher Güter ermöglicht. Andererseits entstehen auch beim Austausch bestimmte Machtpositionen. Derjenige, der Dienste bereitstellt, die dringend benötigt werden, hat gegenüber denen, die diese Dienste benötigen, eine größere Macht.

\subsubsection{Bereitstellung öffentlicher Güter im Austausch gegen soziale Anerkennung}

Ohne dass auf alle Zweige der Konzeptentwicklung eingegangen werden kann ${ }^{14}$, soll hier aus dem Bereich der sozialen Austauschtheorie das Modell von Holländer (1990) zur rationalen Begründung der Entstehung von Normen wegen seines direkten Bezugs zu öffentlichen

\footnotetext{
${ }^{14}$ Zum Beispiel Erklärungen der Kooperation von Olson (1965), Modelle im Rahmen der Altruismusdebatte z. B. von Becker (1974, 1976b), Collard (1978), Arrow (1981), Margolis (1982) und deren Kritik durch Sugden (1982, 1984), Konzepte des iterativen spieltheoretischen Ansatzes bei Hammond (1975), Kurz (1977), Schotter (1981), Axelrod (1981) und deren Kritik z. B. bei Mueller (1986).
} 
Gütern vorgestellt werden.

Heinz Holländer untersucht, ob es möglich ist, dass rational handelnde Wirtschaftssubjekte trotz der oben genannten Schwierigkeiten, öffentliche Güter zu produzieren - kollektiv rationales Verhalten und Kooperation hervorbringen. Holländer geht von der Annahme aus, dass rationales Handeln keine Norm darstellt, sondern im Sinn des Rational-Choice-Ansatzes eine menschliche Grundeigenschaft ist, aus der sich vielmehr erst Normen ergeben. Diese Normen sind gesellschaftlich entwickelte Muster sozialer Interaktionen, die als wünschenswert betrachtet werden. $\mathrm{Ob}$ sich jedoch ein Mensch an sie hält oder nicht, hängt von den Umständen ab.

Normen sind Holländer zufolge Ergebnis eines sozialen Austauschprozesses, in dem Menschen Beiträge zur Erstellung öffentlicher Güter leisten, um dadurch soziale Anerkennung zu erlangen. Die Menschen handeln insofern rational, als sie ihren Gewinn in Form sozialer Anerkennung maximieren. Dabei unterstellt Holländer, dass ein Individuum aufgrund einer Verallgemeinerung ein anderes Individuum sozial belohnt, auch wenn der beobachtete Beitrag die Kollektivgutversorgung nur unwesentlich verbessert. Die subjektive Bewertung eines von einem Individuum $\mathrm{j}$ geleisteten Beitrags $\left(\mathrm{c}_{\mathrm{j}}\right)$ hängt von dem Nutzenzuwachs $\left(\mathrm{U}_{\mathrm{i}}\right) \mathrm{ab}$, den das beobachtende Individuum i erfahren würde, wenn alle N Gruppenmitglieder sich so verhalten würden wie $\mathrm{j}$.

$$
\mathrm{U}_{\mathrm{i}}=\mathrm{N}^{*} \mathrm{c}_{\mathrm{j}} \quad \text { (Gleichung 1.6.1) }
$$

Holländer kann zeigen, dass dieses Konzept der sozialen Anerkennung die Freifahrer-Option unattraktiv macht. Zusammen mit der Annahme von Axelrod (1981), dass mit der Existenz einer Norm immer auch eine Metanorm verbunden ist, die die Anweisung enthält, denjenigen, der die Norm verletzt, zu bestrafen, hat dies zur Folge, dass jemand, der einen Normverstoß beobachtet und diesen nicht bestraft, selbst einen Verstoß begeht und bestraft werden kann. Unser Rechtssystem hat solche Vorschriften institutionalisiert. Wird der Sanktionsmechanismus nicht nur einmal, sondern wiederholt angewandt, so verinnerlicht ihn das Individuum. Am Ende bedarf es einer automatischen Sanktionierung nicht mehr bei jedem Verstoß, da das Individuum die gesellschaftlich anerkannten Maßstäbe 'richtigen' Verhaltens übernimmt und sein Verhalten danach ausrichtet. Holländer sagt allerdings, dass dieses Modell nur unter bestimmten Umständen funktioniert. Zum Beispiel darf es nur eine niedrige Statusorientierung geben, Menschen müssen die emotionale Fähigkeit besitzen, Anerkennung zu vermitteln und es darf keine finanzielle Belohnung für kooperatives Verhalten geben. 


\subsection{Erweiterung des Kapitalmodells durch Identitätskapital und Distinktionsnutzen}

Alle vorgestellten Modelle von Becker et al., Coleman und Holländer haben, um soziale Interaktionen individuell rational $\mathrm{zu}$ machen, Modelle mit Leistung und Gegenleistung entwickelt, welche die Elemente des Sozialen in die individuellen Nutzenüberlegungen integrierbar machen wollen. Konzepte wie Sozial- und Humankapital oder soziale Anerkennung deuten „die Möglichkeiten der theoretischen Endogenisierung weiterer wichtiger Bereiche moderner Gesellschaften an“ (Habisch 1998: 39). Dieser Weg soll im Folgenden genutzt werden, um Aspekte des Individuums selbst und der Bedingungen seines Daseins entsprechend für ökonomische Erklärungsmodelle nutzbar zu machen.

Bei genauerer Betrachtung der ökonomischen Modelllogik existiert eine bisher wenig beachtete Erklärungslücke zwischen dem Individuum und sozialen Interaktionen, die es vor einer entsprechenden Berücksichtigung sozialer Interaktionen in der ökonomischen Modellwelt zu schließen gilt. Alle o. g. Modelle begründen interindividuellen Austausch mit dem für das Individuum daraus resultierenden höheren Nutzen gegenüber der Nichtinteraktion. Dabei ist die Startleistung eines Individuums zu Beginn einer sozialen Interaktion der entscheidende, aber wenig beachtete Schwachpunkt dieser Konzepte. Der Nutzen ergibt sich immer daraus, dass für die erbrachte Startleistung des Individuums A vom Interaktionspartner (Individuum B) eine Gegenleistung erbracht wird und diese auch durch Normen gesichert ist. Dies ist aber in basalen ökonomischen Modellen, in denen es a priori kein Sozialsystem gibt bzw. in das die Kategorie 'andere Individuen' gar nicht eingeführt ist, nicht voraussetzbar. Sobald es Individuen gibt und das Soziale existiert, sind die Erklärungsmuster der gegenseitigen Interdependenzen einschlägig. Es ist innerhalb dieser Modelle aber bisher kaum aufgeklärt, wie aus ihren (einzelnen) individualistischen neoklassischen Modellindividuen soziale Interaktionen und somit Phänomene wie Gesellschaft, Normen, etc. entstehen können.

Aus dem Blickwinkel der Modellindividuen steht jede Startleistung in der Gefahr, nicht durch eine Gegenleistung kompensiert zu werden und somit nur Kosten, aber keinen Nutzen zu generieren. Es gibt einerseits, der ökonomischen Argumentation folgend, für ein Modellindividuum oft keinen Grund, eine empfangene Leistung mit einer Gegengabe zu belohnen. Hier ist vielmehr das Trittbrettfahrerverhalten die ökonomisch rationale Option. Die angebotene Leistung anzunehmen und nichts in die Gegenleistung zu investieren, maximiert den individuellen Nutzen. Andererseits liegt die Entscheidung über die Gegenleistung in der Hand eines anderen 'gleichberechtigten' Individuums. Dies bedeutet, dass a priori damit gerechnet werden muss, dass das andere Individuum genauso nur seiner individuellen Rationalität folgt 
und nicht durch soziale Konstrukte wie Normen, Erfahrungen, etc in seiner Entscheidung gebunden und somit berechenbar gemacht werden kann. Dieses im methodologischen Individualismus, der ökonomischen Rationalität und dem Konzept der Konsumentensouveränität zentral verankerte Konzept verhindert jede Berechenbarkeit der Erwartbarkeit der Gegenleistung. Aufgrund dieser Überlegungen kann es in gegenwärtigen Theorien für ökonomische Individuen nur irrational sein, eine Transaktion oder soziale Interaktion mit einer Startleistung zu beginnen (siehe hierzu auch die ausführliche Analyse bei Euler 2006, Euler/ Freese 2007).

Da es sich insbesondere bei den Modellen von Becker und Coleman um hochabstrakte Theorien handelt, stellt sich die Frage, was sich konkret hinter einer Investitionsrate in Sozialkapital oder was sich hinter der Abschreibungsrate des Sozialkapitals verbirgt. Daher wird im Folgenden zweistufig vorgegangen. Um die vorgeschlagene Theorieerweiterung plausibel zu machen, wird zunächst ein funktionales Modell der direkten sozialen Interaktion zweier rationaler Individuen modelliert, ökonomisch rekonstruiert und hierbei der Distinktionsnutzen eingeführt. Anschließend wird dieses Phänomen in die erweiterte Kapitaltheorie nach Becker integriert und hier als Identitätskapital identifiziert.

Anders als die mittelbaren, indirekten (weil auf eine vom Verhalten anderer Individuen abhängenden Gegenleistung beruhenden) und von äußeren Bedingungen (Normen) abhängigen Anreize der existierenden Modelle zur Generierung sozialer Interaktionen wird der individualistische, neoklassische Modellcharakter über den Distinktionsnutzen mit einem direkten Nutzen für die Aufnahme einer sozialen Interaktion ausgestattet. Dazu wird - aufbauend auf Höllanders Modell des Tausches eines Beitrags zu einem öffentlichen Gut gegen soziale Anerkennung - Distinktionsnutzen als Tausch der durch die soziale Interaktion entstehenden Kosten gegen einen Beitrag zur Verbesserung der eigenen Verfasstheit, der eigenen Identität bzw. des Selbsts in das Konzept der Ökonomie und - als Identitätskapital - in die erweiterte Kapitaltheorie integriert.

Zur Vorbereitung erfolgt zunächst die Darstellung der entsprechenden psychologischen und soziologischen Erkenntnisse und Modelle, die sich mit Identität, Identitätsbildung und -erhalt beschäftigen.

\subsubsection{Psychologische Konzepte von Identität}

Für die angestrebte Betrachtung und Nutzbarmachung sind einige psychologische Konzepte von zentraler Bedeutung. Das klassische psychologische Identitätskonzept untersucht Identitätsfragen im Prozess des Erwachsenwerdens. Erst die jüngere psychologische und sozialpsy- 
chologische Forschung (Keupp et al. 2006, Kellner 1992) sieht einen regelmäßigen „Bedarf“ an Identitätsbildung über das Kindes- und Jugendalter hinaus. Daher werden nach einem kurzen Einblick in das allgemeine psychologische Identitätskonzept, die eng mit dem Postmodernismus in Verbindung stehenden Begriffe der fragmentierten bzw. Patchwork-Identität und die Beurteilung von Identitäten als instabile individuelle Konstrukte (,,identity diffusion“) erläutert.

\subsubsection{Identitätsdiskurse}

Die Werke von E. H. Erikson $(1965,1970,1973)$ stellen immer noch eine aktuelle Brückenfunktion zwischen den verschiedenen Identitäts-Diskursen von Psychoanalyse, Entwicklungsund Sozialpsychologie dar (Kraus 2000: 13). Erikson beschreibt die Entwicklung der Identität als in Stufen vonstatten gehenden Prozess, wobei die Neubildung und Erweiterung auf der Grundlage von vorangegangenen Entwicklungsstufen stattfindet. Dabei sieht er die Entwicklung im Spannungsfeld von Identitätsbildung und Identitätsdiffusion (Erikson 1965: 269, Krauss 2000: 14). Das organisierende und handelnde Ich (Subjekt) steht dem Selbst als veränderliches Objekt z. B. im Bezug auf seine Rolle oder Persönlichkeit gegenüber ${ }^{15}$. Das Selbst wird als Gesamtwahrnehmung der eigenen Persönlichkeit gesehen, es verfügt über eine zeitliche Kontinuität und wird vom Ich in der Bestrebung entwickelt, „,im Wechsel des Geschehens keine allzu großen Gegensätze und Disharmonien innerhalb der eigenen Repräsentanzenwelt aufkommen zu lassen“ (Conzen 1990: 96f). Mead beschreibt diesen Prozess so:

„Identität entwickelt sich; sie ist bei der Geburt anfänglich nicht vorhanden, entsteht aber innerhalb des gesellschaftlichen Erfahrungs- und Tätigkeitsprozesses, das heißt im jeweiligen Individuum als Ergebnis seiner Beziehungen zu diesem Prozess als Ganzem und zu anderen Individuen innerhalb dieses Prozesses.“ (Mead 1968: 177).

Die Weiterentwicklung des Selbstbildes ist abhängig von den Interaktionen mit anderen (Krauss 2000: 17).

Weitere Konzepte beschreiben die Identitätsbildung als strategisches Handeln. Dabei ist Identität ein dynamisches soziales Produkt, das aus personalen Einflüssen wie Erinnerungsvermögen, Bewusstsein oder Denken sowie physikalischen und sozialen Strukturen und Einflussgrößen des sozialen Kontextes entsteht (Breakwell 1989: 190). Breakwell beschreibt

\footnotetext{
${ }^{15}$ Wichtige Begriffe und Konzepte der Psychologie: „Selbst“ und „Selbstrepräsentanz“ bezeichnen ein gedankliches Konstrukt zur Wahrnehmung und Beschreibung der eigenen Person inkl. Körper, Körperteilen, psychischer Organisation und deren Teile (z. B. Hartmann 1950, Kuhn 1964). Der Begriff Identität ist sehr eng mit dem Begriff des Selbst verknüpft, bzw. sogar größtenteils mit ihm deckungsgleich (Oerter/ Montarda 1998: 246). Das „Ich“ ist das handelnde, strukturierte seelische System mit bewussten, vor- und unbewussten Teilen, Gefühlen, Gedanken, Wünschen, physischen und psychischen Verhaltensweisen.
} 
ausführlich, auf welche Strukturen und Prozesse sich Identität zurückführen lässt und wie sie sich manifestiert. Besonders betont sie dabei, dass Identität ein dynamisches soziales Projekt ist, das nicht ohne den sozialen und historischen Kontext verstehbar ist (Breakwell 1989: 9). Andere Autoren sehen Identität als situatives Management, dessen treibende Feder soziale Anerkennung ist (Camilleri 1991). Im Modell von Camilleri spielt die Gesellschaft eine zentrale Rolle für die Zieldefinition der Identitätsbildung und stellt gleichzeitig eine wichtige Ressource dar. Von Sozialkognitivisten wird das Selbst als Eigenwahrnehmung der Summe aller Informationen des Subjektes über sich selbst angesehen (z. B. Selbstkonzept-Forschung, Markus 1977, Krauss 2000: 127), woraus sich u. a. eine Vielzahl von Fragen der Informationsverarbeitung und -verwaltung ergeben.

\subsubsection{Die Postmoderne}

Ohne auf die sehr umfangreiche Diskussion der unterschiedlichen (disziplinären) Zweige des Diskurses um Postmoderne und Postmodernismus einzugehen (zum Einstieg in die Diskussion über Moderne/ Postmoderne siehe z. B. Lash/ Friedmann 1992 oder Beck 1986, Inglehart/ Welzel 2005), sind hier jedoch einige Hinweise nötig, welche gesellschaftlichen und individuellen Phänomene mit Postmoderne im folgenden Kontext verbunden sein sollen.

Auch wenn es ,die Postmoderne' als einheitliches und breit akzeptiertes Konzept nicht gibt, und nicht geben kann, weil sie gerade die Diffusität und das Verschwinden klarer, abgestimmter Definitionen zum Prinzip erhebt, ist postmodernen Gesellschaftsentwürfen das Fehlen von „Metaerzählungen“ (Lyotard 1994) oder, soziologisch gesprochen, das Verschwinden unabdingbaren normativen Strukturen und Wertsysteme (Parsons 1076) gemeinsam. Postmoderne ist ein Label einer historischen Periode mit spezifischen gesellschaftlichen Entwicklungen, Kräften und Beziehungen, es ist auch mit einer spezifischen Art und Weise der Analyse der Welt und Gesellschaft verbunden (Gibbins/ Reimer 1999: 1). Postmoderne Konzepte beeinflussen in erheblicher Weise Wissenschaft, Kunst, Kultur und Sprache, wenn man Wittgenstein und Foucault folgend davon ausgeht, dass die Bedeutung von Wörtern im täglichen Austausch zwischen den Menschen entwickelt und ausgehandelt werden. Ungeachtet der generellen (philosophischen) Inkommensurabilitätshypothese (z. B. Quine 1960) versucht jeder Nutzer von Begriffen, seine individuelle Bedeutungszuschreibung bei der Begriffsbenutzung durchzusetzen. Begriffsinhalte sind nie universell mit einem Begriff verbunden, sondern werden durch ihre Bindung an einen Ort, eine Situation, ein gesellschaftliches und individuelles Umfeld und Wissen bestimmt (z. B. Gibbins/ Reimer 1999: 6). Postmoderne Konzepte gründen somit auf der Erkenntnis, dass weder Sprache und Wissen noch moralische oder ethische Vorstellungen eine absolute Fundierung besitzen und somit 
Richtigkeit und Wahrheit immer relativ und abhängig sind (Derrida 1982, Lyotard 1984, Beck 1986, Rorty 1979, 1989).

Postmoderne bedeutet nicht, dass alle dem klassischen und modernen Zeitalter zugerechneten Phänomene verschwunden sind. Die gegenwärtige Welt beinhaltet aber neben vormodernen und modernen auch solche Phänomene und Prozesse, dessen Bandbreite, Geschwindigkeit und Vielfältigkeit der wirkenden Kräfte es erlauben, anstatt von Moderne von Postmoderne zu sprechen (Hassan 1985: 121, Gibbins/ Reimer 1999). Der wichtigste, die Postmoderne steuernde Prozess, ist die Globalisierung, inkl. der zunehmenden Bedeutung der Massenmedien und der Individualisierung (Beck 1986; sog. Cultural Release z. B. bei Ziehe 1986, genauer siehe unten). Der Zunahme der persönlichen und individuellen Möglichkeiten und Freiheiten und der verstärkten Einforderung von Reflexion und Rationalität stehen eine schwindende gesellschaftliche Orientierungen, schwächer werdende soziale Bindungen und des Verlusts traditioneller Entwicklungsmodelle und dadurch eine neuen individuelle Verletzlichkeit und unbekannter psychischer Druck zur rationalen und, richtigen' Nutung aller sich bietenden Möglichkeiten gegenüber. Das Auftauchen neuer Möglichkeiten und das Verschwinden traditioneller Leitbilder und Traditionen bürdet so jedem einzelnen eine zunehmende alltägliche Entscheidungslast auf, die durch die zunehmenden Unvorhersagbarkeit gegenwärtiger Gesellschaften und die immer schneller ablaufende Entwertung der in der Vergangenheit gemachten Erfahrungen noch verschärft wird und zur Verstärkung des Eindrucks einer neuen aufkommenden postmodernen Zeit beiträgt (Gibbins/ Reimer 1999: 23f). Aufgrund der enormen Vielfalt, der ausufernden, in verschiedenen Kreisen und mit verschiedenen Zielen geführten Debatte um die Postmoderne fehlen ein einheitlicher Rahmen und abgestimmte Definitionen (Gibbins/ Reimer 1999: 22f) ${ }^{16}$. Die folgende ,Definition' stellt aber eine anschauliche Beschreibung der Phänomene dar, die den Übergang von der Moderne zur Postmoderne markieren:

"Modernity ends when words like progress, advance, development, emancipation, liberation, growth accumulation, enlightenment, embitterment, avant-garde loose their attraction and their function as guides to social action." Therbon (1995: 4).

\subsubsection{Das postmoderne Identitätsverständnis}

Diese Prozesse der Postmoderne haben auf der Ebene des Individuums erhebliche Auswirkungen, die unter dem Prozess der Individualisierung zusammengefasst werden (Beck 1986).

\footnotetext{
${ }^{16}$ Für eine Klassifizierung der verschiedenen Postmoderne-Diskurse siehe z. B. Rose (1991) oder Rosenau (1992). Für den Einstieg in die Debatte siehe z. B. Lyon (1994), Smart (1993) oder Crook et al. (1992). Für eine Übersicht siehe z. B. Gibbins/ Reimer 1999: 23-53.
} 
Der Wandel, der sich in den Begriffen Moderne und Postmoderne niedergeschlagen hat, hat auch den Begriff der Identität innerhalb der Psychologie und Sozialpsychologie verändert. In vormoderner Zeit war Identität durch festgelegte Rollen und ein System von Traditionen, Mythen und Religion dem Individuum statisch zugewiesen. Die vormoderne Identität kann als stabil und kaum entwicklungsfähig bzw. -bedürftig betrachtet werden (Kellner 1992, 141, Gibbins/ Reimer 1999, 55). In der Moderne, in die auch Eriksons Werk fällt, wird Identität wähl- und veränderbar, sie wird konstruierbar (Wagner 1995: 232). Infolgedessen wächst die soziale Bezogenheit von Identität, die zur Stabilisierung für das Subjekt nötig ist. Die Postmoderne ist dadurch gekennzeichnet, dass das „Projekt der Moderne“, also die Verheißung, das Subjekt könne sich selbst konstruieren, eine weitere Radikalisierung erfährt und weite Bevölkerungsschichten erfasst. Der bisher gesicherte Platz eines Individuums innerhalb der Gesellschaft und eine erkennbare stabile soziale Ordnung verschwinden zusehends. Dieser Prozess, bereits als Cultural Release (Ziehe 1986) eingeführt, führt zum Verschwinden traditioneller Orientierungen für die Individualentwicklung. Ohne diese durch Normen und Gesellschaft vorgegebenen Entwicklungspfade, entsteht für das Individuum ein erheblicher Mehrbedarf an Selbstkonstruktion von Identität und stellt somit eine ressourcenzehrende Belastung dar (Krauss 2000: 28), die vorher durch eine überschaubare Auswahl an Lebenslaufmodellen vorbereitet war. Wichtigste Eigenschaft der Identität in der Postmoderne ist die Erfahrung ihres Zerfalls, ihrer ständigen Bedrohung und der andauernden Notwendigkeit der situativen Identitätsbildung in einer unberechenbar gewordenen Gesellschaft (Krauss 2000: 32). ${ }^{17}$

Identität in der Postmoderne wird als instabil und flexibel angesehen. Das selbständige, sich selbst entwickelnde und erhaltende Selbst der Moderne wird von postmodernen Theoretikern wie der Frankfurter Schule, Baudrillard u.a. als fragmentiert und im Verschwinden begriffen gesehen (Kellner 1992, 143). Dabei bedeutet instabile, fragmentierte Identität (auch Patchwork-Identität genannt), dass das Selbst nicht mehr ein integriertes und schlüssiges Bild von sich hat, sondern dass seine Identität in vielfach unverbundene Ereignisse und Erlebnisse zerfällt. Identität wird nicht mehr als eine Einheit mit Tiefe, Dauerhaftigkeit und Zusammenhalt wahrgenommen (Jameson 1983, 1984). Patchwork-Identität bezeichnet dabei das Phänomen, dass es nicht mehr , die eine Identität' gibt, die einen ganzen Menschen in allen Lebenssituationen trägt, sondern dass vielmehr aus den vielen unverbundenen Lebensbereichen verschiedene Teilbereiche zur Identitätsbildung beitragen. Diese Beiträge ergeben viele

\footnotetext{
17 Der skizzierte Wandel in der Identität ist auch empirisch z. B. mit dem „Identity Status Interview“ (Übersichtsartikel bei Marcia 1993) nachgewiesen.
} 
Teilidentitäten in spezifischen Kontexten, wie z. B. Beruf, Familie, als Student, etc. und fügen sich nicht mehr kohärent in eine Gesamtidentität ein (Keupp et al. 2006, Krauss 2000).

Das Konzept der Identität ist auch für die Soziologie im Rahmen des soziologischen Interaktionismus von hoher Relevanz. Soziale Beziehungen sind demnach nötig, um ein „Selbst“ bzw. eine „Identität“ aufzubauen. Das soziale Geschehen ist ein offener dynamischer Prozess, der nur durch fortwährende Interaktionen erhalten wird. Jedes Individuum muss sich bzw. seine Identität ständig durch Beteiligung an Interaktionen stabilisieren. Verhalten wird, anders als im Behaviorismus, nicht im Schema von „Stimulus - Response“ erklärt, sondern dadurch, dass der Mensch in einer symbolischen Umwelt lebt und alle Gegenstände, Strukturen, Verhaltensweisen und Personen ihre soziale Bedeutung durch gemeinsame Interpretationen erhalten. Da es sich um eine soziologische Theorie handelt, wird im soziologischen Interaktionismus davon ausgegangen, dass das Soziale, also das Interaktionsgeflecht der Individuen und die inhärenten Werte und Normen dem Individuum vorausgehen (Krappmann 1988: 20f, Rose 1962a, Rose 1962b).

Auch wenn die Nutzung des Begriffs der Identität in der Ökonomie unüblich ist, sind Werte als wichtiges Element der Verhaltenssteuerung ein Baustein der Ökonomie. In der Psychologie erfolgt eine enge Verknüpfung von Identität und Werten (siehe z. B. Mead 1968, Rokeach 1969, Dietz et al. 2005, Schultz 2002). Über die Wirkkette „Werte - Glauben - persönliche Normen - Verhalten“ werden Identität und Werte verhaltensrelevant (Stern 2000).

\subsubsection{Ein funktionales Modell sozialer Interaktionen und das Kon- zept des Distinktionsnutzens}

Die vorgestellten Konzepte des Sozialkapitals reichen noch nicht aus, um die Entstehung sozialer Interaktionen zu erklären. Die Nutzen bringende Wirkung des Sozialkapitals hängt nicht von einem Individuum allein ab, und es besteht eine Unsicherheit, ob Investitionen in soziales Kapital vom Interaktionspartner mit einer Gegenleistung gewürdigt werden. Somit stellt die Chance auf Nutzenstiftung, die von sozialen Beziehungen bzw. SozialkapitalInvestitionen ausgeht, keinen ausreichenden Anreiz für das Modellindividuum dar, sich in einem konkreten Fall für die Investition in eine soziale Interaktion zu entscheiden. Den Kosten der Startleistung steht in sozialen Situationen lediglich die Möglichkeit einer Gewinnrealisierung auf der Basis des dabei gebildeten Sozialkapitals oder die Chance auf eine Gegenleistung gegenüber. Diese Basis reicht für ein rationales Modellindividuum nicht aus, um mit einer Startinvestition in Vorleistung zu treten. 

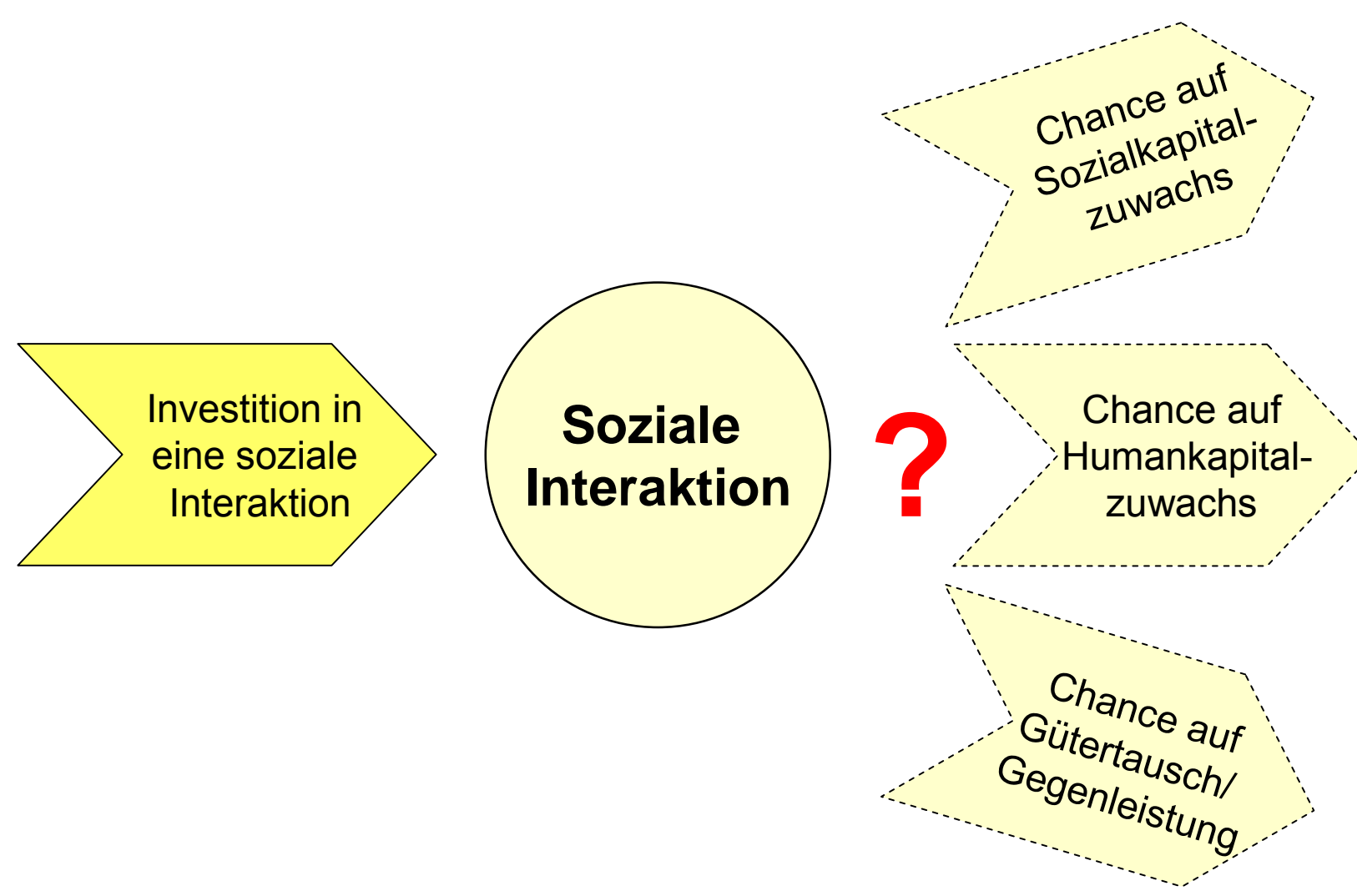

Abb 1.3. Grundlage der funktionalen ökonomischen Rekonstruktion einer Interaktion zwischen zwei Individuen ist die Analyse der Kosten (Investitionen) und des erzielbaren Nutzens in Form von Kapitalrückflüssen (Sozial-, Humankapital oder Güter). Zentrales Problem ist die im Text dargestellte Risikohaftigkeit all dieser möglichen Nutzenkategorien.

Um diese Lücke zu füllen und erklären zu können, warum es zu der beobachteten Vielfalt sozialer Interaktionen und zur Existenz öffentlicher Güter kommt, bedarf es aus Sicht der ökonomischen Kapitaltheorie und dem damit verbundenen Modell des homo oeconomicus eines stärkeren direkten Anreizes für ein Individuum, in soziale Interaktionen zu investieren. Hierzu wird das funktionale Konzept des Distinktionsnutzens vorgeschlagen.

Um die Idee des Distinktionsnutzens plausibel zu machen, sind die psychologischen und soziologischen Anforderungen, die ein Individuum braucht, um ein Individuum sein zu können, zu betrachten. Das Wort Individuum ist ein Differenz- bzw. Abgrenzungsbegriff. Um gemäß der (philosophischen) Logik existieren zu können, muss ein Individuum von etwas, z. B. seiner Umwelt, abgegrenzt sein. Gibt es nur die beiden Entitäten Individuum und Umwelt (so wie es das einfachste Modell des homo oeconomicus vorsieht), ist das Individuum alleine, es gibt keine anderen Individuen. Damit ergibt der Individuumsbegriff keinen Sinn und es kann auch nichts Soziales geben.

„Hier wird aber darüber hinaus behauptet, daß jeder, um den strukturellen Erfordernissen nach Identität nachkommen zu können, auf eine bestimmte Art sozialer Beziehungen angewiesen ist, nämlich auf Beziehungen in denen Erwartungen übernommen oder auch abgelehnt werden 
können.“(Krappmann 1988: 35).

„Einer allein kann nicht individuell sein, da die eigene Individualität sich bereits auch dem Umstand verdankt, immer schon mit anderen zu sein. (...) Die eigene Individualität ist sinn- und darstellungs-logisch mit der nichtreduzierbaren Individualität anderer Personen verschränkt. Wenn alle anderen das Gleiche auf die gleiche Weise täten, dann wäre die eigene Weise nicht mehr individuell zu nennen." (Abel 1999: 349).

„,Der Einzelne hat eine Identität nur im Bezug auf die Identität anderer Mitglieder seiner gesellschaftlichen Gruppe.“(Mead 1973: 206)

Spencer-Brown fasst die grundlegende Bedeutung des Unterscheidungsprozesses zusammen, indem er behauptet, der Anfang von allem sei die Unterscheidung:

„We take as given the idea of distinction and the idea of indication, and that we can not make an indication without drawing a distinction" (Spencer-Brown 1973: 1).

Soll es mehrere Individuen geben, so müssen diese „aus der Umwelt“ herausgelöst werden und vom ersten Individuum unterschieden werden (eine ausführliche philosophische und logische Herleitung siehe Euler 2006). Jedes Individuum braucht „Merkmale“ oder „Eigenschaften“, die es von anderen Individuen unterscheidet. Dies kann schon alleine die Benennung als „anderes Individuum“ sein. Die Merkmalszuweisung entsteht bei Kontakten zwischen zwei Individuen, z. B. als „Geber“ und „Nehmer“ und generiert so durch Merkmalsaustausch den Distinktionsnutzen für die Individuen. Der Nutzen entsteht, da individuelle Merkmale Grundlage des Individuum-Seins sind und die postmoderne Identitätsforschung jedem Individuum eine ständige Notwendigkeit zur Identitätspflege zuschreibt. Da die oben dargestellte Unterscheidung vom anderen Individuum in jedem Fall, also beispielsweise auch bei einem nicht erfolgreichen Gütertausches und auch im Fall einer ,im üblichen Sprachgebrauch als negativ verlaufenen Interaktion“ wie etwa in Form eines Streites erfolgt, resultiert aus jeder interindividuellen Interaktion jedem der Interaktionspartner ein Distinktionsnutzen (U (D) bzw. D).

Durch die Investition eines Individuums in eine soziale Interaktion entsteht ihm sofort ein Distinktionsnutzen, und zusätzlich ergibt sich die Chance auf Entstehung von Human- und Sozialkapital und die Erlangung von Gütern. Die Interaktion selbst wird dabei als Anerkennung der Existenz anderer Individuen und als Akt des Unterscheidens angesehen. Distinktionsnutzen ist der Wert, der entsteht, wenn mit der Unterscheidung Merkmale, Eigenschaften bzw. Informationen zwischen den interagierenden Individuen ausgetauscht werden. Jede Unterscheidung weist dem initiierenden Individuum und dem Interaktionspartner Merkmale 
und Eigenschaften zu.

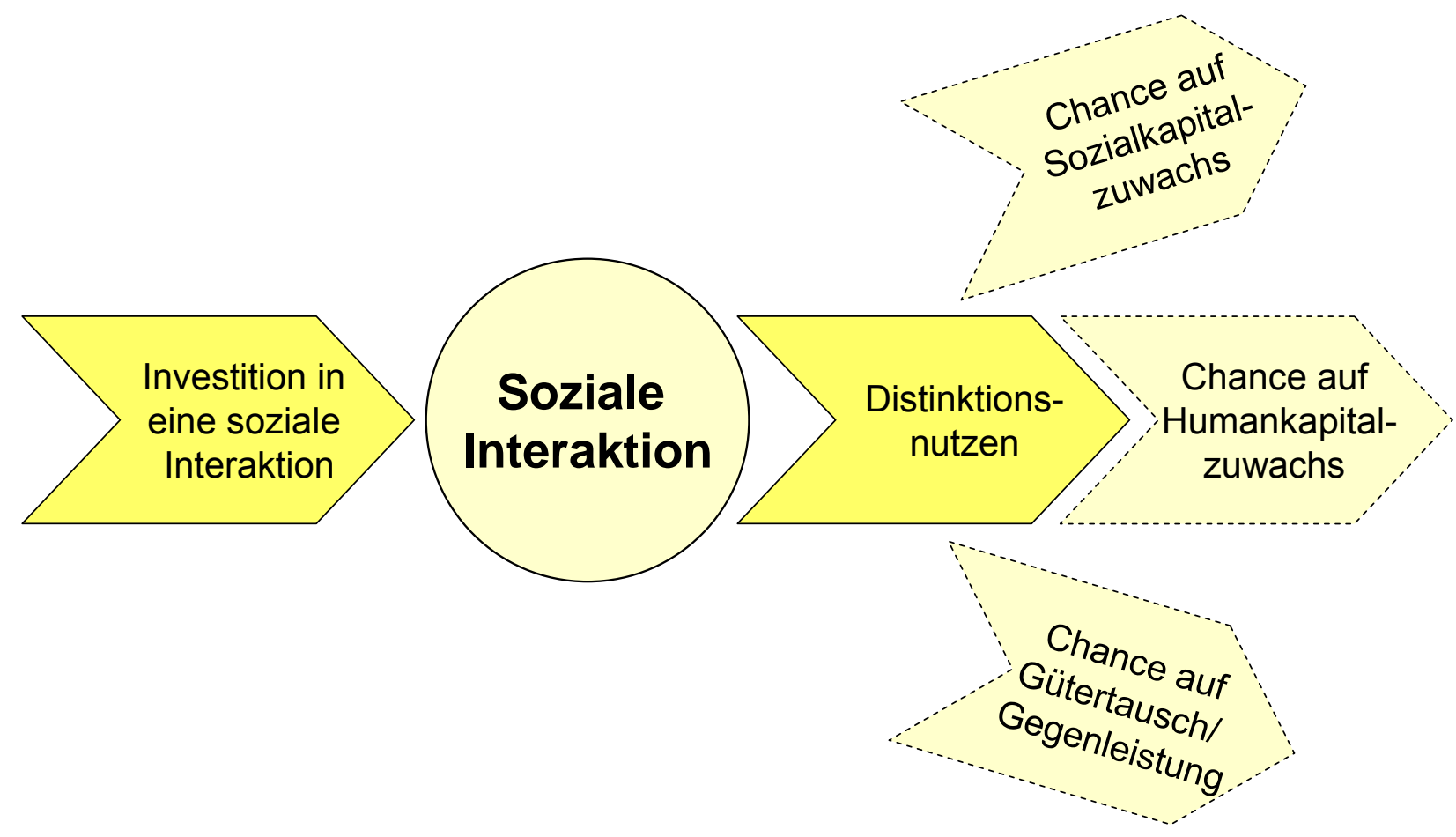

Abb. 1.4.: Die Einführung des Distinktionsnutzens - hergeleitet aus der logischen und psychologischen Notwendigkeit mit anderen Individuen in der Umwelt zu interagieren, um Individualität und Identität entstehen zu lassen - führt im funktionalen Modell der Interaktion zweier Individuen zu einer für das initiierende Individuum kalkulierbaren Rückfluss seiner Investitionen in Form des Nutzens eines Beitrags zur Entstehung bzw. Entwicklung seiner Identität. Da dieser Nutzen auf dem Prozess der Unterscheidung beruht, wird er vom engl. distinction (Unterscheidung) ,Distinktionsnutzen' genannt.

Überträgt man dieses Konzept auf soziale Interaktionen, so konkretisiert sich dieser Nutzen für den homo oeconomicus darin, dass er durch die Unterscheidung - also den Interaktionsprozess - zum Individuum wird und dabei mit dem anderen auf diese Weise entstandenen Individuen Merkmale wie „Käufer“, „Nutzer“, „Tauschpartner“, „Besitzer eines Gutes“, etc. austauscht. Der Distinktionsnutzen kann in diesem funktionalen Modell als Konstitution des Individuums durch Merkmalszuweisung angesehen werden.

Gegenüber der Anpassung des Präferenzsystems z. B. durch Einführung einer Präferenz für Altruismus oder soziale Kontakte bietet das Konzept des Distinktionsnutzens den Vorteil, als strukturell notwendiges Merkmal eines Individuums von Seiten der Theorie und Logik herleitbar zu sein und, da er als Grundlage eines individuellen Kapitalstocks dient, Identitätsfragen mit den ökonomischen Instrumenten in Entscheidungssituationen zu integrieren. Damit wird nicht behauptet, dass z. B. der Altruismusansatz falsch sei oder nicht der Realität entspräche. Es wird aber für das ökonomische Modell eine Theorie-Variante angeboten, die neben der Beobachtung der Veränderung des Identitätskonzeptes, konsequent dem Beckerschen Forschungsprogramm der Aufdeckung von relevanten Restriktionen des Handelns und 
von intrinsischen Kostenkategorien folgt.

\subsubsection{Identitätskapital}

Die Übertragung dieses funktionalen Modells der sozialen Interaktion und dem dabei entstehenden Distinktionsnutzen für die interagierenden Individuen in die abstrakten ökonomischen Theorien, insbesondere die erweiterte Kapitaltheorie, erfolgt durch Einführung einer neuen Kapitalart. Der Distinktionsnutzen, der in einer Zeitperiode erworben wurde, verschwindet entsprechend nicht, sondern wird als Identitätskapital 'gespeichert' und kann somit verhaltensrelevant werden. Das Identitätskapital fasst den Bestand eines Individuums an, in sozialen Interaktionen erworbenen identitätsbildenden Merkmalen und Eigenschaften, zusammen.

\subsubsection{Identität und Ökonomie}

Da die Behandlung von Identität und Interaktion mit einer ökonomischen Konnotation hier nicht zum ersten mal stattfindet, werden zunächst anderen Konzepte mit Bezug auf Identitätskonzepte vorgestellt, bevor das Konzept des Identitätskapital erläutert, in die erweiterte Kapitaltheorie integriert und schließlich in die Theorie der öffentlichen Güter eingefügt wird.

Akerlof und Kranton (2005: 12, 2002, 2000) setzen die Identität einer Person in den Kontext der Existenz gesellschaftlicher Verhaltensnormen und definieren dann Nutzen und Kosten der Identität als Nutzen und Kosten, die durch identitätskonformes bzw. nicht identitätskonformes Verhalten entstehen. Sie nutzen dieses Konzept, um Anreize innerhalb von Organisationen mittels der Principal-Agent-Diskussion zu erklären, welche weiteren Anreizmethoden es neben den monetären gibt, den Erwartungen seines Dienstherren zu entsprechen. In Akerlof und Kranton (2002) wird dieses Modell auf schulische Prozesse angewendet. Es wird dargelegt, dass es beim Schulbesuch nur teilweise um Wissenserwerb (Humankapitalerwerb) geht und Aspekte der jugendlichen Identitätsbildung ebenfalls das schulische Verhalten bestimmen. Auch Wichardt (2006) modelliert die Vorteile der Existenz einer Präferenz für identitätsgerechtes Verhalten innerhalb eines sozialen Austauschmodells zur Entstehung von Kooperation und sozialen Normen. Benabou/ Tirole (2004) entwerfen ein Agentenmodell, in dem sie, um prosoziales Verhalten zu erzeugen, neben dem materiellen Nutzen die individuellen Aspekte von Altruismus, sozialer und individueller Identität integrieren. McCall/ Simmons beschrieben 1966 ein „Handeln um Identität“:

"The moves of each party are motivated by cost-reward considerations but take the form of insinuations about identities. At base, that is, the negotiation is a process of bargaining or haggling over the terms of exchange of social rewards, yet it does not assume the outward appearance of a crude naming of prices. Rather, it takes the form 
of an argument or debate over who each person is; the tactics of rhetorical persuasion or dramatic arts are more evident in the process than are those of the market place." (McCall/ Simmons 1966: 141).

Diese Ansätze der Berücksichtigung von Identitätsaspekten in ökonomischen Kontexten erlauben zusammen mit den vorgestellten Auswirkungen, die die Postmoderne auf das Identitätsverständnis hat und mit der oben vorgeführten funktionalen ökonomischen Rekonstruktion sozialer Interaktionen, Identität als weitreichende, sehr basale Nutzen- und Kostenkategorie einzuführen. Im Rahmen dieser Arbeit wird daher vorgeschlagen, Identitätsaspekte eines Individuums als Identitätskapital den anderen Kapitalarten des Individuums (Güter, Geld (materielles Kapital), Human- und Sozial- bzw. persönlichem Kapital) beiseite zu stellen. Die vorgestellten Erkenntnisse machen es plausibel, dass für ein ökonomisches Modellindividuum in sozialen Kontexten und Umwelten, Aspekte der eignen Identität, Identitätsabgrenzung und Identitätsentwicklung von hoher Bedeutung sind.

\subsubsection{Eigenschaften des Identitätskapitals}

Als Identitätskapital wird die Summe aller Eigenschaften bzw. Merkmale eines Individuums angesehen, die dieses in Interaktionen mit anderen Individuen erworben bzw. zugewiesen bekommen hat. Das Identitätskapital bildet - übertragen auf psychologische Konzepte - auf der einen Seite die Identität des Individuums, also das von ihm bei anderen erzeugte Bild und seine Selbstwahrnehmung, ab. Auf der anderen Seite stellt es das Wissen über Merkmale und Eigenschaften anderer Individuen dar. Wenn die soziale Interaktion der ,Tausch' der Merkmale bzw. Informationen durch eine Unterscheidung (Distinktion) ist, dann stellt das Identitätskapital den daraus resultierenden Kapitalstock dar.

Jedes Individuum benötigt, um agieren zu können, zunächst eine Grundausstattung mit Identitätskapital. Es muss ein Individuum unter vielen anderen Individuen und ausgestattet mit individuellen Merkmalen und Eigenschaften konstituiert sein.

$$
\begin{aligned}
\frac{d I}{d x}>I_{\min } & \quad \text { (Gleichung 1.7.1) } \\
\left(\operatorname{oder}^{\frac{\delta I}{\delta x}}>I_{\min }\right) &
\end{aligned}
$$

Diese Anforderung an eine Mindestausstattung ist allerdings nur in Extremsituationen relevant und vergleichbar mit der Mindestausstattung eines Individuums mit Nahrungsmitteln, Wärme und Wasser. Ein Mangel an Mindestausstattung führt zum bekannten Wertparadoxon, 
dass in der Wüste bei akutem Wassermangel, Wasser problemlos mit Diamanten oder Gold aufgewogen werden kann. Das Identitätskapital (I) wird im Fall der fehlenden Mindestausstattung als Konkurrenz zur Nutzenfunktion U in Entscheidungssituationen modelliert:

$$
E=f(U, I) .
$$

(Gleichung 1.7.2)

Dabei fällt I in die Kategorie der Grundbedürfnisse, die in Entscheidungssituationen unterbewusst bzw. weniger rational mit der Nutzenfunktion konkurriert.

Generell geht die ökonomische Theorie aber davon aus, dass die Grundbedürfnisse des Individuums gedeckt sind und es um die rationale am individuellen Wohlbefinden orientierte Optimierung des Ressourceneinsatzes geht. Es soll daher hier so verfahren werden, wie üblicherweise mit den anderen Grundbedürfnissen im ökonomischen Kontext auch. Wenn die Mindestanforderungen gedeckt sind, ist Identität wie Ernährung, Wärme und Wasser in die individuelle Nutzenkalkulation integriert. Der Kauf von Lebensmitteln oder die Anforderungen und Gestaltung der Wohnung sind so ökonomisch handhabbar. Entsprechend zielen Investitionen eines Individuums in seine Identität auf eine Verbesserung seiner Ausstattung mit Identitätskapital.

Als abstrakter Kapitalstock, werden nun soziale Interaktionen und andere identitätsbildende Aktivitäten in die Kapitaltheorie integriert. Das Identitätskapital kann sich mit Humankapital (Wissen und Informationen über die Welt und andere Individuen) und Sozialkapital (spezielle Verbindung bzw. Verbundenheit zu anderen Individuen) überschneiden, genauer gesagt, es beeinflusst diese beiden. Humankapital kann in sozialen Interaktionen erworben werden, z. B. in Form von Wissen über andere Individuen und entsteht somit zunächst als Identitätskapital, das dann in Humankapital (individuelle Wissensressource) umgewandelt wird. Sozialkapital kann ausschließlich in sozialen Interaktionen erworben werden. Funktional gesehen geht Identitätskapital der Sozialkapitalbildung und z. T. der Humankapitalbildung voraus. Es ist aber durchaus auch möglich, Identitätskapitalaspekte unter die Beckerschen Humankapitalia zu subsumieren. Die hier vorgenommene Separierung ist nicht unbedingt nötig, dient hier aber der Explizierung und Prononcierung der Position von Identitätsfragen innerhalb der Theorie. So, wie das Humankapital die Aspekte des individuellen Wissens und das Sozialkapital die Aspekte des individuellen sozialen Netzes für die Ökonomie nutzbar macht, so stellt das Identitätskapital die philosophisch-logische Notwendigkeit und die psychologischen und soziologischen Erkenntnisse über Identität in ökonomisch handhabbarer Art und Weise bereit. Identitätskapital verhält sich analog der anderen Kapitalarten der Kapitaltheorie. Identitätskapital (I) hat einen abnehmenden Grenznutzen. Mit zunehmender Ausstattung mit Identitätska- 
pital nimmt der individuelle Nutzen einer weiteren Einheit I ab. Die Höhe der Kapitalbestände und die Kosten für eine Einheit zur Aufstockung des jeweiligen Kapitalstocks bestimmen, wie das Individuum seine Ressourcen einsetzt. Identitätskapital ist dabei durch andere Kapitalarten substituierbar. Es ist z. B. denkbar, sich zur Stärkung der Identität materielle Güter mit Statusfunktion (Auto, Schmuck, Kleidung, etc.) zuzulegen ${ }^{18}$. Umgekehrt kann Identitätskapital in Güter, Human- und Sozialkapital getauscht werden, indem das Wissen, das in sozialen Interaktionen über andere Individuen erworben wurde eingesetzt wird, um Güter, Wissen oder Kontakte zu erlangen.

Eine Verzinsung von Identitätskapital kann man sich, übertragen ins funktionale Modell, als den Zugewinn an I vorstellen, der generiert wird, wenn das Individuum sein Identitätskapital als Human- oder Sozialkapital einsetzt. Dies kann dann zu zusätzlichen sozialen Interaktionen führen, die neue Merkmale verleihen können. Umgekehrt unterliegt Identitätskapital einem schleichenden Verlust dadurch, dass Teile der Selbst- und Fremdwahrnehmung nach einer bestimmten Zeit verblassen und als nicht mehr angemessen bewertet werden.

\subsubsection{Auswirkungen sozialer Interaktionen auf die Nutzenfunktion}

Unter Rückgriff auf die Gleichung 1.4.3, die erweiterte Nutzenfunktion mit den Bestimmungsgrößen konsumierte Güter (y, z), Persönliches Kapital (P) und Sozialkapital (S),

$$
\mathrm{U}=\mathbf{U}\left(\mathrm{y}_{\mathrm{t}}, \mathrm{z}_{\mathrm{t}}, \mathbf{P}_{\mathrm{t}}, \mathrm{S}_{\mathrm{t}}\right), \quad \text { Gleichung (1.7.3) }
$$

wird nun dargestellt, wie die Berücksichtigung von Aspekten der sozialen Interaktion (si) als Mittel der Identitätsstabilisierung und Identitätsbildung die Nutzenfunktion beeinflussen kann.

Die Einflüsse einer sozialen Interaktion auf die Nutzenfunktionen lassen sich zunächst unterscheiden in

(1) einen direkten Nutzen für den Individualitätsstatus des Individuums in Form des Distinktionsnutzens,

(2) einen ersten indirekten Nutzen der Interaktion auf den individuellen Bestand an Identitätskapital (I)

(3) einen zweiten indirekten Nutzen der Interaktion auf den individuellen Bestand an

\footnotetext{
${ }^{18}$ Hierbei wird auch deutlich, dass mit zunehmender Komplexität der Kapitaltheorie die eindeutige Zuordenbarkeit von Gütern zu Kapitalarten verschwindet. So hat ein entsprechendes Auto einen Anteil materiellen Kapital (der Besitz des Gutes) und einen Anteil Identitätskapital (die soziale Zuweisung eines Images durch soziale Wahrnehmung des Autobesitzes). Eine weitere Analyse zeigt, dass es zur Generierung und Nutzung eines Statussymbols speziellen Human- (das Wissen, das etwas einen bestimmten Status verleiht) und Sozialkapitals (die Menschen, die einem die gewünschte Statuswahrnehmung entgegenbringen) bedarf.
} 
Sozialkapital (S). Infolge der Erhöhung des Identitätskapitals kann es auch zu einer Erhöhung des Sozialkapitals kommen.

(4) einen dritten indirekten Nutzen der Interaktion auf den individuellen Bestand an persönlichem Kapital (P). Auch hier gilt, dass diese Wirkung doppelt indirekt ist, da es erst infolge der Erhöhung des Identitätskapitals auch zu einer Erhöhung des persönlichen Kapitals kommen kann.

Die Eigenschaften des Interaktionspartners sind von erheblicher Bedeutung für die mögliche Kapitalbildung. Dies wird über die soziale Interaktion (si) repräsentiert.

Sozialkapital kann nur in sozialen Interaktionen gebildet werden. Der Nutzenzuwachs hängt neben der Ausgangshöhe von S auch vom Identitätsstatus, also dem Identitätskapital I ab. Dies spiegelt wider, dass nicht einfach jede soziale Interaktion das individuelle Sozialkapital erhöht, sondern dies davon abhängt, wer ich bin und welcher Art und Intensität die Interaktion ist. Dies wird in Gleichung 1.7.4 durch den Term S (I) bzw. vollständig S ( I (si) ) repräsentiert. Auch im Bereich des persönlichen Kapitals (P) gibt es sehr viele Elemente, die durch soziale Kontakte vermittelt werden. Dies sind z.B. Kenntnisse über andere Personen oder Wissen und Fertigkeiten, die man von anderen Personen im Rahmen von sozialen Interaktionen erlernt hat und die zu den eigenen Erfahrungen gehören. Sie werden durch P (I) bzw. vollständig P ( I (si) ) repräsentiert.

Damit ergibt sich für die Erweiterung der Beckerschen erweiterten Kapitaltheorie um Aspekte der Identität folgende Nutzenfunktion zum Zeitpunkt t:

$$
\mathrm{U}=\mathbf{u}\left(\mathrm{y}_{\mathrm{t}}, \mathrm{z}_{\mathrm{t}}, s i_{\mathrm{t}} \mathbf{I}_{\mathrm{t}}\left(s \mathrm{i}_{\mathrm{t}}\right), \mathbf{P}_{\mathrm{t}}\left(\mathrm{I}_{\mathrm{t}}\left(s \mathrm{i}_{\mathrm{t}}\right)\right), \mathrm{S}_{\mathrm{t}}\left(\mathrm{I}_{\mathrm{t}}\left(s \mathrm{i}_{\mathrm{t}}\right)\right)\right)
$$

(Gleichung 1.7.4)

Da Sozialkapital als reines soziales Gut nur in sozialen Internaktionen gebildet werden kann, zum persönlichen Kapital jedoch auch viele Elemente zählen, die unabhängig von Internaktionen entstehen, beeinflusst der Identitätskapitalbestand S stärker als P:

$$
\frac{\delta P}{\delta I}<\frac{\delta S}{\delta I}
$$

(Gleichung 1.7.5)

mit den konsumierten Gütern y, z und den Beständen an persönlichem (P), Sozial- (S) und Identitätskapital (I) sowie der sozialen Interaktion $s_{t}$.

Zur weiteren Vereinfachung der weiteren Betrachtung werden P und S zum Humankapital zusammengefasst:

$$
\mathrm{U}=\mathbf{u}\left(\mathrm{y}_{\mathrm{t}}, \mathrm{z}_{\mathrm{t}}, s \mathbf{i}_{t}, \mathbf{I}_{\mathrm{t}}\left(s \mathbf{i}_{t}\right), \mathbf{H}_{\mathrm{t}}\left(\mathrm{I}_{\mathrm{t}}\left(s \mathrm{i}_{t}\right)\right)\right)
$$


Es ergibt sich somit für die Abhängigkeit des Nutzens von der sozialen Interaktion:

$$
\frac{\delta U}{\delta s i}=\frac{\delta u}{\delta s i}+\frac{\delta U}{\delta I} * \frac{d I}{d s i}+\frac{\delta U}{\delta H} * \frac{d H}{d I}
$$

(Gleichung 1.7.7).

Die einzelnen Terme der Nutzenfunktion haben folgende Eigenschaften: Der individuelle Nutzen U einer sozialen Interaktion (si) ist immer positiv ist:

$$
\frac{\delta U}{\delta s i}>0
$$

(Gleichung 1.7.8).

Der von sozialer Interaktion abhängige Identitätskapital-Nutzen ist in einem definierten Bereich ebenfalls nach Gleichung 1.7.9 stets positiv:

$$
\frac{\delta U}{\delta I} * \frac{d I}{d s i}>0
$$

(Gleichung 1.7.9).

unterliegt aber einem abnehmenden Grenznutzen.

Für die im Humankapital ausgelösten Nutzenzuwächse gilt,

$$
\frac{\delta U}{\delta H} * \frac{d H}{d I}>0
$$

sobald

$$
\mathbf{I}>\mathbf{I}_{\text {schwelle }}
$$

(Gleichung 1.7.10),

(Gleichung 1.7.11).

Erst bei Überschreiten einer Schwelle setzt die Bildung von Humankapitalkomponenten wie S und $\mathrm{P}$ ein.

Daraus folgt, dass die Gesamtgleichung 1.7.7 stets positiv ist und der Gesamtnutzen einer sozialen Interaktion dort positiv ist, wo dieser Nutzenbetrag den Kostenbetrag überschreitet. Abbildung 1.5. illustriert diesen Sachverhalt grafisch. $I_{\text {schwelle }}$ stellt dabei den Punkt dar, an dem die Humankapitalbildung und damit ein zusätzlicher Nutzen einsetzt.

Die Nebenbedingungen der Gleichung 1.7.6 sind (1) die Zeitrestriktion:

$$
\mathbf{T}=\mathbf{t}_{\mathrm{si}}+\mathbf{t}_{\text {Arbeit }}+\mathbf{t}_{\text {alleine_ohne_si }}
$$

(Gleichung 1.7.12)

Das Gesamtzeitbudget $\mathrm{T}$ wird genutzt für die Zeit der sozialen Interaktion $\left(\mathrm{t}_{\mathrm{si}}\right)$, für Erwerbsarbeit $\left(t_{\text {Arbeit }}\right)$ und weitere Zeit ohne soziale Interaktionen $\left(t_{\text {alleine_ohne_si }}\right)$.

\section{(2) Budgetrestriktion:}

$$
B=\text { Lohnansatz } L * t_{\text {Arbeit }}=\text { Kosten der Konsumgüter } y, z
$$

(Gleichung 1.7.13)

Das monetäre Gesamtbudget B, ergibt sich aus dem Lohnansatz multipliziert mit der Arbeitszeit und wird genutzt, um Konsumgüter zu erwerben. 


\subsection{Identitätskapital, Distinktionsnutzen und öffentliche Güter}

Es soll nun gezeigt werden, dass das Konzept des Distinktionsnutzens und Identitätskapitals zwei Dinge leisten kann. Es kann zunächst die ökonomischen Anreize zur sozialen Interaktion für ökonomisch rational handelnde Modellindividuen bereitstellen, die die Grundlagen des Sozialen sind. Das Konzept erweist sich weiterhin als nützliches Instrument, um einen Beitrag zur ökonomischen Betrachtung öffentlicher Güter zu leisten.

\subsubsection{Entstehung des Sozialen}

Abbildung 1.5. verdeutlicht, wie das Konzept des Identitätskapitals zur Bevorzugung sozialer Interaktionen gegenüber der Nicht-Interaktion bei rationalen Individuen führt. Es stellt bei immer gleichartiger Interaktion zweier Individuen die Abhängigkeit von Nutzen und Kosten von der Investitionshöhe des initiierenden Individuums dar. Dabei fällt bei der Summenkurve ein Bereich geringen, aber von 0 verschiedenen Investments in eine soziale Interaktion auf (Bereich A in Abb. 1.5.), der für das investierende Individuum immer nutzenstiftend ist. Dem Identitätskapitalzuwachs infolge des Distinktionsnutzens stehen nur geringe Investitionen gegenüber. Es ergibt sich selbst dann für das initiierende Individuum ein positiver Nutzensaldo, wenn die Interaktion zu keinem weiteren Nutzen in Form von Güteraustausch oder Sozialkapitalgewinn führt.

Dies entspricht der empirischen Beobachtung der enormen Vielzahl und Vielfalt sozialer Interaktionen. Die Existenz bzw. Entstehung sozialer Strukturen, die auch für die ökonomische Behandlung der öffentlichen Güter nötig sind, baut somit auf einem rationalen Anreiz zu sozialen Interaktionen auf.

Der Weg, der von der einzelnen Unterscheidung (Distinktion) zum aus Individuen bestehenden Sozialgefüge führt, soll nur ganz kurz skizziert werden. Da ein Individuum einen direkten Nutzen aus dem Kontakt zu anderen Individuen hat (Distinktionsnutzen und Identitätskapitalbildung), ist es notwendig für das Individuum, die Kategorie „Umwelt“ zu unterteilen in „andere Individuen“ und „Umwelt“. Somit ergibt sich für ökonomische Modelle die Notwendigkeit, anderen Individuen eine gesonderte Stellung einzuräumen und zwar zunächst nicht, weil sie den homo oeconomicus mit Gütern oder Dienstleistungen versorgen können, sondern weil die soziale Interaktion, die nur mit anderen Individuen möglich ist, zur Erschaffung, Existenzsicherung und zur Weiterentwicklung der Identität des homo oeconomicus notwendig ist. Auf dieser Grundlage ist es für individualistische Modelle rational handelnder Individuen bereits möglich, die Entstehung komplexer sozialer Phänomene zu verstehen. Der Distinkti- 
onsnutzen ist der Anreiz, um (1.) andere Individuen in der Umwelt überhaupt als Individuen wahrzunehmen und (2.) mit diesen in Kontakt zu treten und zwar, ohne dass es um eine Güter- oder Dienstleistungsbeschaffung im klassischen Sinne geht, sondern nur aus dem Grund der Verbesserung des eigenen Identitätsstatus (Distinktionsnutzen bzw. Identitätskapital).

Stehen am Anfang (logisch betrachtet) nur singuläre Distinktionen (also konkret isolierte Interaktionen mit Individuen in der Umwelt, die nicht voneinander unterschieden sind), so erweitert sich die Vielfalt der möglichen Merkmale durch die Verknüpfung dieser einzelnen Distinktionen $\mathrm{zu}$ Identitäten und somit zu bestimmten und wiedererkennbaren Individuen. Diese exponentielle Vermehrung der Merkmale durch Erhöhung der Komplexität (z. B. zeitliche Ordnung von Distinktionen und Zuordnung einzelner Distinktionen zu Individuen) ist der Anreiz zu komplexeren Interaktionen. Unterscheidet man die anderen Individuen nicht untereinander, so ist jeder Tausch nur ein einfacher Tausch und verleiht - vereinfacht gesprochen - nur das Merkmal „Tauschpartner“. Sind jedoch verschiedene Individuen eingeführt (genauer unterschieden von der Umwelt), so kann aus einfachen Tauschaktionen eine Vielzahl von Merkmalen abgeleitet werden: „Tauschpartner von Individuum 1 “, „Tauschpartner von Individuum 2 “, etc. Dies erhöht das individuelle Identitätskapital und stellt die Grundlage der Entstehung komplexer Strukturen und komplexen sozialen Verhaltens dar ${ }^{19}$.

Erst in der zweiten, hierauf aufbauenden Konzeptstufe geht es um die Befriedigung der klassischen Bedürfnisse im Bereich von Gütern und Dienstleistungen. Indem das Individuum einzelne soziale Interaktionen verschiedenen Individuen zuordnet und in eine zeitliche Abfolge ordnet, entsteht aus der Interaktion ein Kapitalstock, der als Identitätskapital bezeichnet wird. Identitätskapital bezeichnet zunächst die eigenen Merkmale und Eigenschaften, die bei anderen bekannt sind sowie Merkmale und Eigenschaften anderer Individuen, über die man in Interaktionen Kenntnis gewonnen hat. Um dieses Kapital zu vermehren, kann das Individuum einerseits viele eher oberflächliche und kurze Kontakte mit anderen Individuen initiieren oder anderseits die Kontakte zu einzelnen Individuen intensivieren und vertiefen.

\footnotetext{
${ }^{19}$ Es sei angemerkt, dass dieses Konzept einen Lösungsansatz für das ,,second-order-free-rider-Problem“ (Heckathorn 1996) bereithält. Das „second-order-free-rider-Problem“ besagt, dass im Rahmen der existierenden Modelle zur Behandlung und Erklärung öffentlicher Güter - insbesondere zur Erklärung der Nichtanwendung der Trittbrettfahreroption (engl. free-rider-option) - Normen, soziale Kontrolle und Sanktionen nötig sind. Diese stellen aber selbst ein öffentliches Gut dar bzw. besitzen den Charakter eines öffentlichen Gutes, da sie existieren und von jedem in Anspruch genommen werden können, es aber nicht nötig ist, selbst zu ihnen durch normkonformes Verhalten beizutragen. Somit sind entsprechende Lösungsmodelle nicht konsistent, da sie die Existenz des öffentlichen Gutes „Normen“ als Voraussetzung benötigen, um die Entstehung öffentlicher Güter zu erklären. Auch bei dem vorliegenden Konzept wird zur Erklärung der Existenz öffentlicher Güter auf das Vorhandensein des Sozialen mit seinen Normen und Sanktionen zurückgegriffen. Die Entstehung des Sozialen kann allerdings im Rahmen des Modells des Distinktionsnutzens Theorie-konform und Theorie-intern erfolgen.
} 
Identitätskapital wird entweder in Form des öffentlichen Bildes, das das Individuum in den Interaktionen erzeugt hat (Aufmerksamkeit, soziale Achtung, Vertrauenswürdigkeit, etc.) oder als Human- und Sozialkapital (d. h., das Individuum weiß aufgrund der sozialen Interaktionen, wer welche Dienstleitung anbietet, wer welche Güter besitzt, wer vertrauenswürdig ist, etc.) in klassischen Marktsituationen wirksam.

Zusätzlich zu dem Nettonutzen-Bereich A (Abb. 1.5.) gibt es einen weiteren Bereich höherer Investitionen in eine einzelne soziale Interaktion, die dem Individuum ebenfalls einen Nettonutzen stiftet (Bereich B in Abb. 1.5.). Dies ist dann der Fall, wenn die Summe aus Distinktionsnutzen und Nutzen der Identitätskapitalbildung die Investitionskosten übertrifft. Der abnehmende Grenznutzen zunehmenden Identitätskapitals führt dazu, dass sich höhere Investitionen in die soziale Interaktion nicht auszahlen. Für den Fall einer bereits guten Ausstattung mit Identitätskapital und einem infolgedessen geringen Nutzenzuwachses kann es auch Individuen geben, die zu einem bestimmten Zeitpunkt keinen Bereich B besitzen und für die sich somit höhere Investition in soziale Interaktion nicht Nutzen vermehrend auswirken. Dies ist gegeben, wenn die Kurve des Nutzens durch Human-/Sozialkapitalzuwachs (in Abb.1.5. grün dargestellt) erst bei einer höheren Investition beginnt, eine geringere Anfangssteigung aufweist oder früher eine Sättigung eintritt.

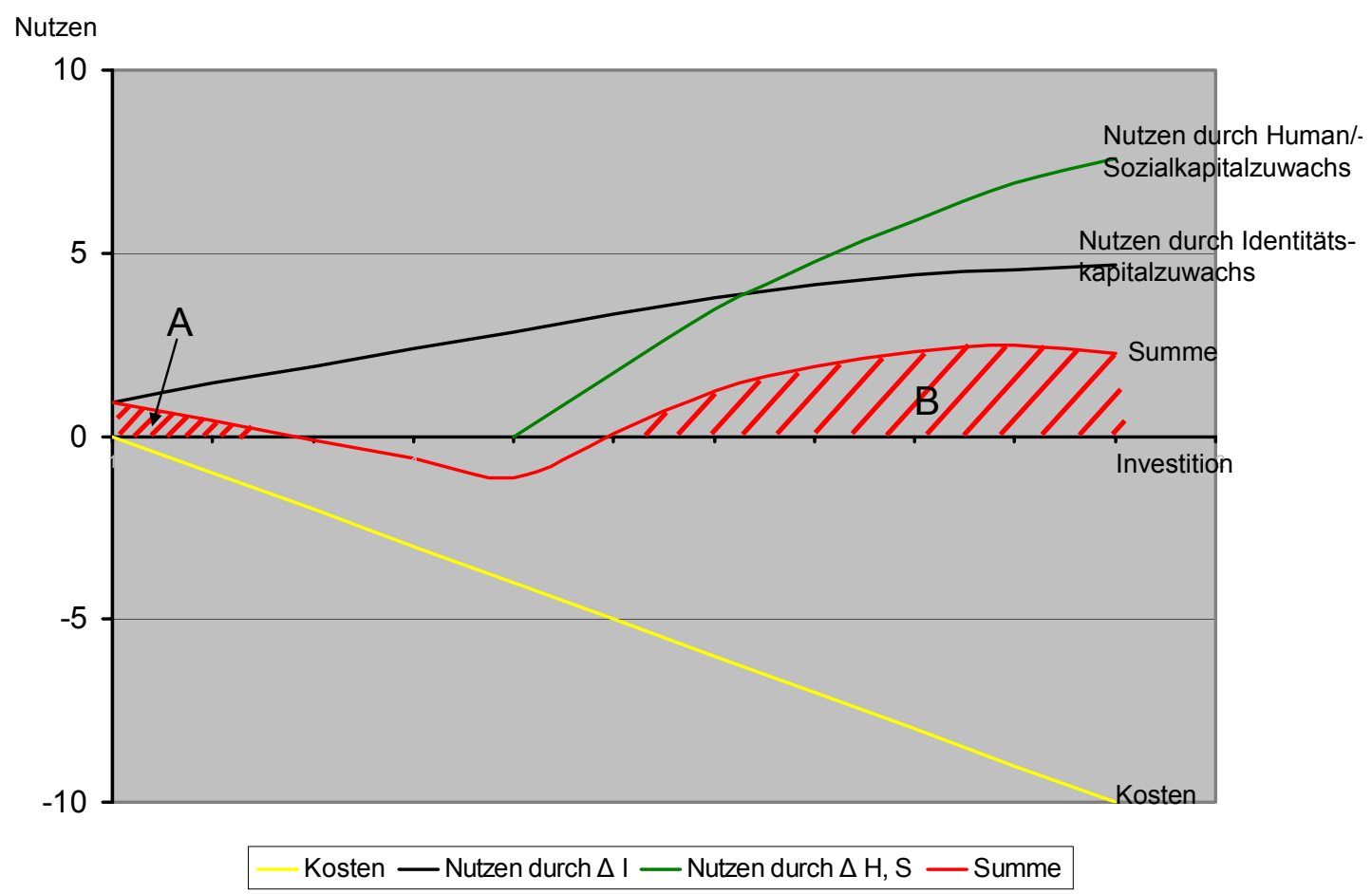

Abb.1.5.: Die Darstellung von Nutzen und Kosten einer einmaligen (immer ähnlichen) sozialen Interaktion eines Individuums mit einem zweiten Individuum. Den Kosten (Investitionen) einer sozialen Interaktion stehen der Nutzen aus dem Identitätskapitalzuwachs (I) und der erst ab einem Schwellwert einsetzende Sozialkapitalzuwach (S), gegenüber. Jede soziale Interaktion erzeugt I. Daher beginnt der Nutzen von I auch bei kleinsten Investitionen in die soziale Interaktion bereits im positiven Bereich. Je höher die Investitionen in die Interaktion ist, desto höher ist der Zuwachs bei I. S hingegen setzt erst mit einer gewissen Mindestinvestition ein, 'Kleinstinteraktionen' führen nicht zur Erhöhung von S. I und S 
erreichen ab einer bestimmten Investitionshöhe eine Sättigung. Die Summation dieser Kosten und Nutzen ergibt zwei Bereiche (A, B), in denen dem betrachteten Individuum aus der sozialen Interaktion ein Nettonutzen entsteht. Für eine ausführliche Erklärung siehe Kap. 1.8.1.

Eine Illustration dieser zwei „Nettonutzen-Bereiche“ liefert die Alltagserfahrung. Sich mit unbekannten Tischnachbarn $\mathrm{zu}$ unterhalten ist nicht aufwendig, bereitet aber Freude. In anderen Fällen investiert man mehr Zeit in die Vorbereitung und scheut auch nicht den mit einer Theater- oder Esseneinladung verbundenen Zeit- und Kostenaufwand, um jemanden besser kennen zu lernen. Diese höhere Investition steht dann, ökonomisch gesprochen, in der Absicht, den Eingeladenen besser kennen zu lernen, bei ihm ein vollständigeres Bild zu hinterlassen und so über mehr „Merkmale“ eine Erhöhung des Identitätskapitals zu erreichen oder Anerkennung zu ernten bzw. ihn dem eigenen sozialen Netz und somit dem eigenen Sozialkapital hinzuzufügen.

\subsubsection{Entstehung öffentlicher Güter bei Berücksichtigung des Distinktionsnutzens}

\subsubsection{Das klassische Dilemma}

Klassischerweise wird das Trittbrettfahrerproblem bei öffentlichen Gütern ökonomisch so erklärt, dass der egoistische Nutzer nur den Nutzen aus dem öffentlichen Gut (xöffGut) in Anspruch nimmt, seinen Nutzen also aus seinem Güterbestand ( $\left.\mathrm{x}_{\text {Bestand }}\right)$ und dem Nutzen des öffentlichen Gutes generiert und somit folgende Nutzenfunktion resultiert:

$$
\mathrm{U}_{\text {egoist }}=\mathbf{f}\left(\mathrm{X}_{\text {ÖffGut }}, \mathrm{X}_{\text {Bestand }}\right) \quad \text { (Gleichung 1.8.1). }
$$

Bei ursprünglich gleicher Kapitalausstattung sollte ein „,kooperativer Nutzer“, der zum Erhalt des Gutes beiträgt und dadurch sein eigenes materielles Kapital ( $\left.\mathrm{x}_{\text {Bestand }}\right)$ um diesen Beitrag zur Erhaltung des öffentlichen Gutes ( $c_{\text {Beitrag }}$ ) verringert, einen geringeren Nutzen erzielen. Wenn zusätzlich gilt, dass $\mathrm{c}_{\text {Beitrag }}$ den Bestand des öffentlichen Gutes nicht zu ändern vermag, würde folgen

$$
\mathrm{U}_{\text {koop. }}=\mathrm{f}\left(\mathrm{X}_{\text {ÖffGut }}, \mathrm{X}_{\text {Bestand }}, \mathrm{C}_{\text {Beitrag }}\right) \quad \text { mit } \quad \mathrm{x}_{\text {Bestand }}=\mathrm{X}_{\text {Bestand (t-1) }}-\mathrm{C}_{\text {Beitrag }} \text { (Gleichung 1.8.2) }
$$

und damit stets

$$
\mathbf{U}_{\text {egiost }}>\mathbf{U}_{\text {koop. }} \quad \text { (Gleichung 1.8.3) sein. }
$$

Somit würde gemäß klassischer ökonomischer Theorie das öffentliche Gut nicht erhalten werden bzw. wäre gar nicht entstanden. Dieses klassische ökonomische Dilemma lässt sich nur unter Zuhilfenahme des Sozialen auflösen. Den bekannten Lösungen einer sozialen Kontrolle oder einer Präferenz für soziales bzw. altruistisches Verhalten oder eines Nutzens aufgrund von altruistischem Verhalten soll hier das Konzept des logisch und psychologisch fundierten Distinktionsnutzens entgegengestellt werden. 


\subsubsection{Kooperativer Nutzer mit Identitätskapitalzuwachs}

Wenn die Leistung des kooperativen Nutzers in einem sozialen Kontext stattfindet, wird sein Nutzen zwar weiterhin um seinen eigenen Beitrag $\left(\mathrm{c}_{\text {Beitrag }}\right)$ verringert, der kooperative Nutzer erhält aber zusätzlich vom Interaktionspartner einen Nutzen in Form eines Identitätsnutzenzuwachses $(\Delta \mathrm{I}(\mathrm{si}))$ infolge des Distinktionsnutzens:

$$
\mathrm{U}_{\text {koop. }}=\mathrm{f}\left(\mathrm{X}_{\text {ÖffGut }}, \mathrm{X}_{\text {Bestand, }}, \mathrm{C}_{\text {Beitrag }}, \Delta \mathrm{I}(\mathrm{si})\right) \quad \text { (Gleichung 1.8.4) }
$$

Aus den Gleichungen 1.8.1., 1.8.2. und 1.8.4. ist unmittelbar ersichtlich, dass kooperatives Verhalten in diesem Modell dann individuell rational ist, wenn der erzielte Identitätskapitalzuwachs $(\Delta \mathrm{I}(\mathrm{si}))$ die Nutzeneinbuße des eigenen Beitrags $\mathrm{c}_{\text {Beitrag }}$ mindestens kompensiert. Die weiteren Auswirkungen der sozialen Interaktion und der Identitätskapitaländerung werden hier nicht betrachtet (siehe hierzu Kapitel 1.7.3.3 oder Gleichung 1.7.4).

\subsubsection{3. Öffentlicher egoistischer Nutzer mit Identitätskapitalzuwachs und sozialen Kosten}

Für den Fall, dass der Egoist kein heimlicher Konsument sein will und auch an einem Distinktionsnutzen z. B. in Form sozialer Aufmerksamkeit oder über die Eigenschaftszuschreibung „Egoist“, „rücksichtsloser Nutzer“, etc. interessiert ist, kann ihm die Entstehung eines Distinktionsnutzens nicht abgesprochen werden, da dieser immer in einer sozialen Situation entsteht. Es ist aber zu beachten, dass die dazu notwendige soziale Interaktion umgehend auch soziale Strafen mit sich bringen wird. Die Berücksichtigung sozialer Strafen ist in diesem Konzept ökonomisch zu rechtfertigen, da mit dem Distinktionsnutzen der grundlegende Mechanismus der Entstehung sozialer Strukturen Teil des Konzepts ist.

Der stille, unbeobachtete Konsum eines öffentlichen Gutes ist möglich, nicht jedoch die „heimliche“ Erlangung von Distinktionsnutzen und Identitätskapital. Da dieser immer mit einer sozialen Interaktion verbunden ist, unterliegt er den Regeln sozialer Kontrolle und Bestrafung (auf der Grundlage von gesellschaftlichen Normen). Es ergibt sich somit für die öffentliche egoistische Nutzung:

$$
U_{\text {egoist,öffentlich }}=f\left(x_{\text {ÖffGut }}, x_{\text {Bestand, }}, \Delta \mathrm{I}(\mathrm{si}), \mathrm{g}_{\mathrm{sozStrafe}}\left(\mathrm{S}, \mathrm{y}_{\text {sozDist }}\right)\right. \text { ) (Gleichung 1.8.5). }
$$

Dabei stellt $\mathrm{g}_{\text {sozStrafe, }}$ dessen Größe abhängig ist vom Sozialkapital S des Individuums und von der sozialen Distanz ( $\mathrm{y}_{\text {sozDist }}$ ), z. B. eine Verminderung des Sozialkapitals, ein Vertrauensverlust oder die Erhöhung der Transaktionskosten bei zukünftigen Interaktionen für den öffentlich nutzenden Egoisten dar. Die soziale Distanz zwischen den Interaktionspartnern repräsentiert die Wahrscheinlichkeit und Härte, mit der der Interaktionspartner den Egoisten direkt bestraft oder in späteren Interaktionen benachteiligt. Somit ist die Nutzenfunktion des 
öffentlich agierenden Egoisten von der Summe des Gebrauchswertes des öffentlichen Gutes und dem Identitätskapitalzuwachs, abzüglich der sozialen Kosten bzw. der sozialen Bestrafung bestimmt.

Einen empirischen Hinweis auf dieses Verhalten bietet z. B. jugendliche Aufmüpfigkeit, gezielter Regelbruch, öffentliche Provokation, etc. Hier geht es für die Personen darum, Aufmerksamkeit „um jeden Preis“ zu erregen und somit Distinktionsnutzen und einen Identitätskapitalzuwachs zu generieren. Die einhergehende gesellschaftliche Missbilligung wird in Kauf genommen oder einkalkuliert.

Es lässt sich aus dem Konzept des Distinktionsnutzens und Identitätskapitals keine systematische Bevorzugung sozialen kooperativen Verhaltens ableiten. Da jedoch das Konzept soziale Interaktionen fördert und die Entstehung des Sozialen plausibel macht, erscheint es im Rahmen dieses Konzeptes legitim, es für die ökonomische Betrachtung sozialer Normen zu nutzen. Es bleibt weiterhin von der individuellen Kapitalausstattung abhängig, ob ein Individuum zu einem öffentlichen Gut beiträgt oder nicht. Der Nutzen der sozialen Interaktion, soziale Anerkennung bzw. Bestrafung können jetzt aber treffsicherer modelliert werden.

\subsection{Erklärungswert von Distinktionsnutzen und Identitätskapital}

Wenn die Triebfeder für soziale Interaktionen in einem ökonomischen Modell in Präferenzen, also z. B. in einer Präferenz für soziale Interaktionen gesehen wird, hat dies zwei gravierende Nachteile. Einerseits ist ökonomischen Modellen die Bildung der Präferenzen unzugänglich. Andererseits sind Präferenzen per Definition als über die Zeit konstant gesetzt ${ }^{20}$. Mit der Modellierung der Triebfeder des Sozialen als Präferenz nimmt sich ein Modell die Möglichkeit, aus Operationalisierungen des Sozialen (z. B. Frequenz, Zählung oder Dauer von sozialen Interaktionen) auf das Verhalten schließen zu können, da Präferenzen äußeren Einflüssen und einer genaueren ökonomischen Betrachtung unzugänglich sind. Daher bietet es sich an, Identität und Distinktion, logisch und psychologisch unbestreitbare Notwendigkeiten eines Individuums, als Fließgrößen (Kapitalstöcke bzw. Nutzen) zu definieren. Dabei wird hier dem Vorgehen Beckers bei der Integration von Erfahrungen, Erziehung, etc. eines Individuums für dessen zukünftiges Verhalten gefolgt, da Becker, um diese Faktoren modellmäßig fassbar zu machen, das persönliche Kapital einführte. Indem er vergangene Erfahrungen als veränderbare Fließgröße darstellt, macht er Erziehung, Prägung und persönli-

\footnotetext{
${ }^{20}$ Die Setzung erfolgt aus produktiven (methodologischen) Gründen und ist notwendig, um Verhaltensänderungen im Zeitverlauf über Güterausstattungen zu erklären, andernfalls ändert sich das Verhalten einfach deshalb, weil sich mit der Zeit die Präferenzen ändern. Die Aufgabe der Annahme stabiler Präferenzen würde die Erklärungskraft ökonomischer Modelle zerstören (Becker 1976: 5, Stigler/ Becker 1977: 76, Becker 1996: 6).
} 
che Erfahrungen z. B. aus vorangegangenen Entscheidungen für die ökonomische Entscheidungsfindung des Modellindividuums verfügbar (Becker 1996: 7). Mit der Einführung von Distinktionsnutzen und Identitätskapital geschieht dies analog für die grundlegenden Individuum-schaffenden und -erhaltenden Prozesse der sozialen Interaktion.

Wichtig ist $\mathrm{zu}$ betonen, dass dieses Konzept nicht in Konkurrenz $\mathrm{zu}$ den existierenden ökonomischen Konzepten steht. Für den Bereich einmaliger, anonymer Marktsituationen ergibt dieser Ansatz aufgrund der fehlenden Bedeutung der sozialen Komponenten keine Modifikationen der mengen- bzw. preisabhängigen Gleichgewichtstheorien. Auch wird die Gültigkeit etwa der Institutionenökonomie oder des Transaktionskostenansatzes nicht in Frage gestellt. Diese werden lediglich schlüssiger an die individualistische neoklassiche Theorie angebunden. Abb. 1.6. zeigt, dass sich das Identitätskapital, genauso wie das Sozialkapital dort bewähren, wo zur klassischen Marktsituation soziale Interaktionen treten.

\subsubsection{Distinktionsnutzen und Identitätskapital in verschiedenen Lebenssituationen}

Angelehnt an die empirischen Hinweise, die Glaeser et al. (2002) für die Plausibilität des Sozialkapitalkonzeptes geben, sollen im Folgenden einige Überlegungen die Plausibilität und Nutzbarkeit des vorgestellten Konzeptes aus Distinktionsnutzen und Identitätskapital verdeutlichen.

Der Bedarf an sozialen Interaktionen und damit an Distinktionsnutzen und Identitätskapital ist im Leben eines Menschen nicht konstant. Im Kindes- und Jugendalter erreicht das Bedürfnis nach Identitäts(kapital)entwicklung einen Höhepunkt. Das Schließen und Aufgeben von Freundschaften und die Rebellion in der Pubertät als Abgrenzung des Individuums durch (oft negative) Rückmeldungen anderer Individuen schaffen die Grundausstattung des Individuums mit Identitätskapital.

Nach Ausbildung und Familiengründung nimmt das Bedürfnis nach neuen Sozialkontakten und auch die Intensität der Pflege alter Beziehungen in der Regel ab, da man durch die Einbettung in stabile soziale Institutionen (Ehe und Familie, Berufsumfeld, Nachbarschaft etc.) eine kontinuierliche und regelmäßige Identitätserhaltung (Distinktionsnutzen) sichergestellt hat und über einen gefestigten Stock an Eigenschaften und Merkmalen (Identitätskapital) verfügt.

Im höheren Lebensalter steigt vielfach das Bedürfnis nach neuen sozialen Kontakten wieder an, wenn man durch den Tod von Freunden, Partnern, Verwandten und Nachbarn zunehmend von den alten Sozialkontakten und damit vom gewohnten regelmäßigen Distinktionsnutzen 
abgeschnitten wird. Man begibt sich in Kirchen-, Bridge-, oder Seniorenkreise, beginnt mit Kulturreisen oder Seniorensport, um neue soziale Kontakte zu knüpfen, Quellen für Distinktionsnutzen zu generieren und aktuelles Identitätskapital zu erlangen.

Die Intensität der sozialen Interaktionen hängt auch stark mit der eigenen Lebenssituation zusammen. Lebt man in einem stabilen und bekannten sozialen Umkreis, sind die Aktivitäten mäßig, das Interesse an neuen sozialen Kontakten gering. Man erlangt im täglichen sozialen Umgang regelmäßig Distinktionsnutzen und besitzt einen stabilen Satz an Identitätskapital. Hat sich aber die Lebenssituation z. B. durch die Trennung vom Partner oder den Umzug in eine neue Stadt geändert, fehlt der regelmäßige Distinktionsnutzen, da man weniger soziale Kontakte hat. Auch ist das Identitätskapital z. T. dadurch entwertet, dass mit dem Partner oder dem alten sozialen Umfeld auch die geteilten sozialen Eigenschaften und Merkmale der eigenen Identität verloren gegangen sind. Daher hat man einen hohen Anreiz, neue Kontakte zu knüpfen, ist also bereit, Zeit und andere Ressourcen in neue soziale Kontakte zu investieren.

\subsubsection{Nutzen einer sozialen Interaktion in Abhängigkeit von der sozialen Nähe zum Interaktionspartner}

Die absolute Höhe des Distinktionsnutzens einer einzelnen sozialen Interaktion ist im funktionalen Modell unabhängig von der sozialen Nähe der Interaktionspartner. Der Nutzen der Unterscheidung des eigenen Individuums durch Abgrenzung und Unterscheidung (Distiktion) von einem anderen Individuum entsteht immer und würde daher in Abb. 1.6. als Gerade erscheinen $^{21}$. Der Nutzen ist jedoch nicht in jeder Situation konstant hoch, sondern hängt z. B. vom individuellen Bestand an Identitätskapital ab. Ist dieser Bestand gering, so ist der Distinktionsnutzen einer sozialen Interaktion höher, ist der Bestand an Identitätskapital (I) hoch, so ist der Distinktionsnutzen, den das Individuum aus der sozialen Interaktion zieht, geringer.

Dem gegenüber ist der Nutzen einer einzelnen sozialen Interaktion für seine Teilnutzenkomponenten I und Sozialkapital (S) durchaus abhängig von der sozialen Nähe der Interaktionspartner (siehe Abb. 1.6.). Identitätskapital wird dabei mit zunehmender sozialer Nähe der Interaktion zunehmend Nutzen stiftend, da die Wiedertreffwahrscheinlichkeit und die Interaktionshäufigkeit ansteigen. Der Nutzen, der aus dem Sozialkapital gezogen wird, ist ebenfalls abhängig von der Wiedertreffwahrscheinlichkeit und Interaktionshäufigkeit, aber

\footnotetext{
${ }^{21}$ Da sich der Distinktionsnutzen D jedoch auf das funktionale Modell bezieht und I und S Kapitalarten innerhalb der Kapitaltheorie darstellen, kann streng genommen keine gemeinsame Darstellung in einem Diagramm erfolgen.
} 
auch von der bestehenden Integration des Interaktionspartners in das eigene soziale Netz. Daher gibt es im Randbereich des eigenen sozialen Netzes ein Nutzenmaximum, das dadurch entsteht, dass neue Individuen dem eigenen Netz hinzugefügt oder schwache Verbindungen z. B. durch Vertrauensbildung gestärkt werden. Der Nutzen einer Sozialkapitalinvestition im engeren sozialen Netz ist hingegen geringer als in der Netzperipherie, da hier eine Verbesserung der bestehenden Beziehungen kaum noch möglich ist. Interpretiert man das Sozialkapital zwischen zwei Individuen als die Wahrscheinlichkeit, dass Individuum 1 über Individuum 2 eine Leistung erhält, so kann diese Wahrscheinlichkeit bei den besten Freunden, bei Partnern oder Familienmitgliedern kaum noch erhöht werden.

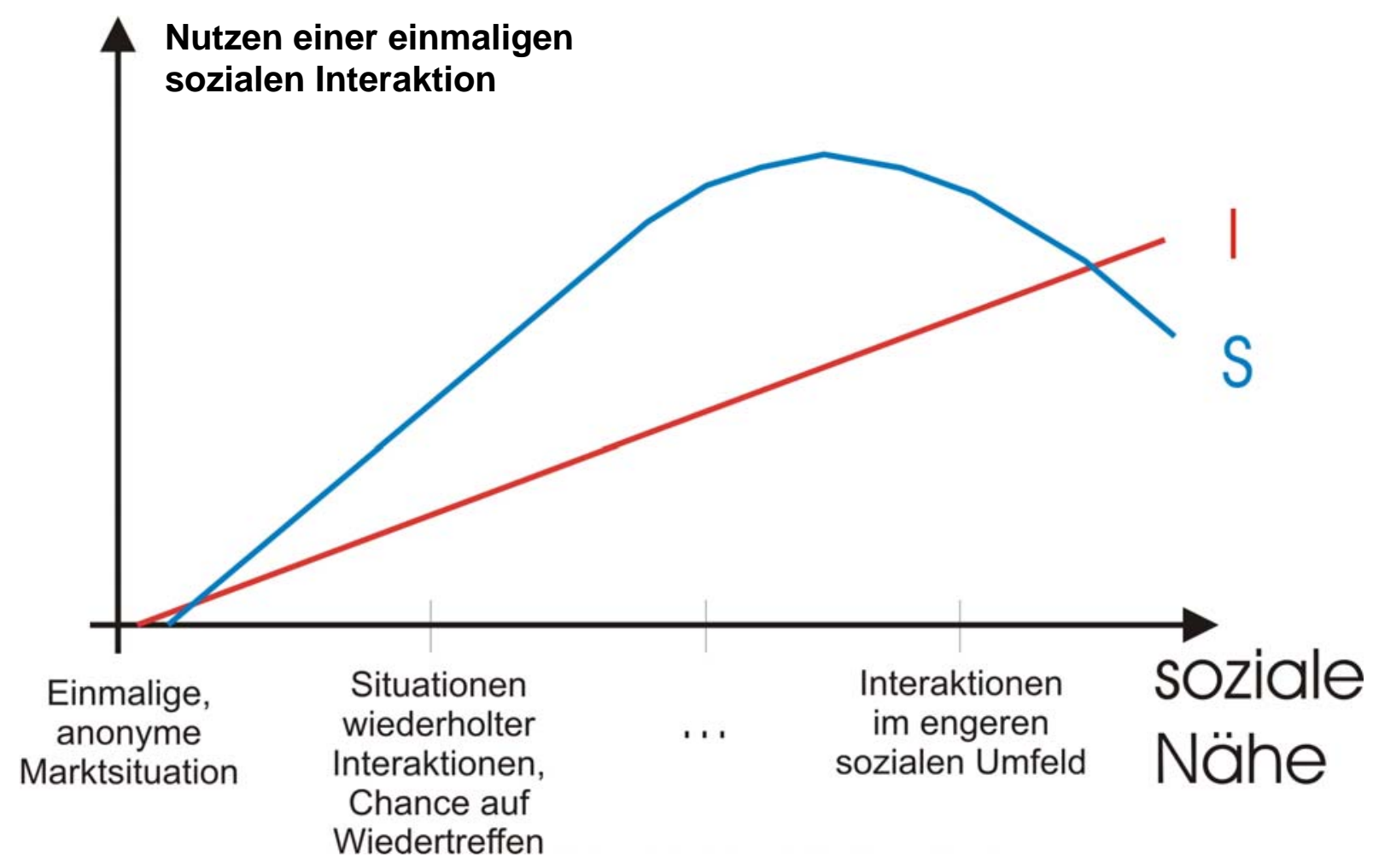

Abb. 1.6.: Abhängigkeit der Nutzenhöhe vom Grad der sozialen Nähe der an der Interaktion beteiligten Individuen. Erläuterungen siehe Kap. 1.9.2. S - Nutzen des Sozialkapitalzuwachses, I - Nutzen des Identitätskapitalzuwachses.

\subsection{Fazit: Identitätskapital und Distinktionsnutzen als konstitutive Merkmale eines ökonomischen Indivi- duums}

1. Die logische Analyse des individualistischen neoklassischen Modellindividuums macht es erforderlich, neben der Umwelt und dem eigenen Ich auch andere Individuen aus der Umwelt abzugrenzen. Nur dann werden der Begriff und das Konzept des ökonomischen Individuums plausibel. Da „Individuum“ ein Differenzbegriff ist, ergibt es keinen Sinn, von einem isoliert existierenden Individuum in der Welt $\mathrm{zu}$ sprechen. Ein Individuum 
kann es nur in Abgrenzung zu anderen Individuen geben. Dieser Prozess der Abgrenzung, im logischen Sinn eine Unterscheidung mit Eigenschafts- bzw. Merkmalszuweisung, im konkreten Sinn eine Interaktion, erzeugt den hier mit Distinktionsnutzen bezeichneten Nutzen der Individuumsabgrenzung.

2. Der Schritt von Modellen der Ein-Individuen-Ökonomie zu Mehr-Individuen-Modellen geht mit komplexen Fragen der Interdependenz zwischen diesen Individuen einher. In der klassischen Ein-Individuen-Ökonomie interagieren andere Individuen nur indirekt mit dem betrachteten Individuum, indem die anderen auf die Preise und Mengen von Gütern wirken und diese dann das Individuum in seiner Entscheidung beeinflussen. In MehrIndividuen-Ökonomien, wie Becker und Coleman sie entwerfen, werden die Handlungen und Handlungsmöglichkeiten der anderen Individuen in die individuellen RationalChoice-Überlegungen einbezogen, nicht zuletzt, da die anderen Individuen durch Mitgliedschaft im sozialen Netz Teil des eigenen Sozialkapitals sein können. Coleman lässt zwar die Berücksichtigung der Strategien der Vertrauensgewährung und Kooperation bzw. Vertrauensverweigerung und Defektion in den eigenen Überlegungen zu, schließt aber eine Berücksichtigung der Folgen, die das eigene Sozialverhalten bei anderen Individuen hat, aus. Bei Becker finden sich im Rahmen seiner Soziobiologie- und Altruismusdiskussion (Becker 1976) Überlegungen zur Integration der Reaktion des anderen auf das eigene Verhalten. Die Erweiterung der Modelle um Distinktionsnutzen und Identitätskapital implementiert zusätzlich die Interdependenz der Existenz anderer Individuen mit dem Individuum als Ganzem, indem die Existenz anderer Individuen erst das „IndividuumSein“ des betrachteten Individuums ermöglicht und so direkt auf seine Präferenzen, seine Ressourcen (Identitätskapital) und sein Weltmodell Auswirkungen hat.

3. Identität wird in den Gesellschafts- und Sozialwissenschaften seit der Postmoderne nicht mehr als statische und gesellschaftlich zugewiesene Rolle angesehen, sondern als eine in sozialen Interaktionen erworbene, fragile, wandlungs- und entwicklungsbedürftige Selbstkonstruktion des Individuums. Empirische Forschung sowie psychologische und soziologische Theorien sehen eine Zunahme der individuellen Beschäftigung mit der Aufrechterhaltung und Entwicklung der eigenen Identität. Diese Erkenntnisse machen es plausibel, in sozialen Situationen Prozesse und Aufwand der Identitätsentwicklung auch für ökonomische Theorien nutzbar zu machen.

4. Das Konzept von Distinktionsnutzen und Identitätskapital folgt dem ,Beckerschen Forschungsprogramm', relevante Restriktionen ökonomischen Verhaltens zu identifizierten. Beckers zentrales Anliegen ist die Erweiterung und Aufwertung des Begriffs der Re- 
striktionen. Er gestattet neben den, objektiven', leicht messbaren Restriktionen (wie z. B. Budgets, Gütermengen und Preise) auch Restriktionen in Form intrinsischer Opportunitätskosten (Pies 1998: 16-20). In diesem Sinn sind Distinktionsnutzen und Identitätskapital als neue intrinsische Kostenkategorie anzusehen, die zentrale Funktionen als Restriktionen im Bereich sozialer Interaktionen darstellen.

5. Ein Distinktionsnutzen entsteht bei jeder Unterscheidung (im konkreten Kontext bei jeder sozialen Interaktion) aufgrund der dabei entstehenden Eigenschafts- bzw. Merkmalszuweisung. Der Distinktionsnutzen erklärt in einem funktionalen Modell zur Rekonstruktion sozialer Interaktionen als direkt wirksame Nutzenkategorie, warum Individuen Vorteile aus sozialer Kontaktaufnahme ziehen, auch wenn sie Zeit oder andere Ressourcen dafür aufwenden (Abb. 1.7.).

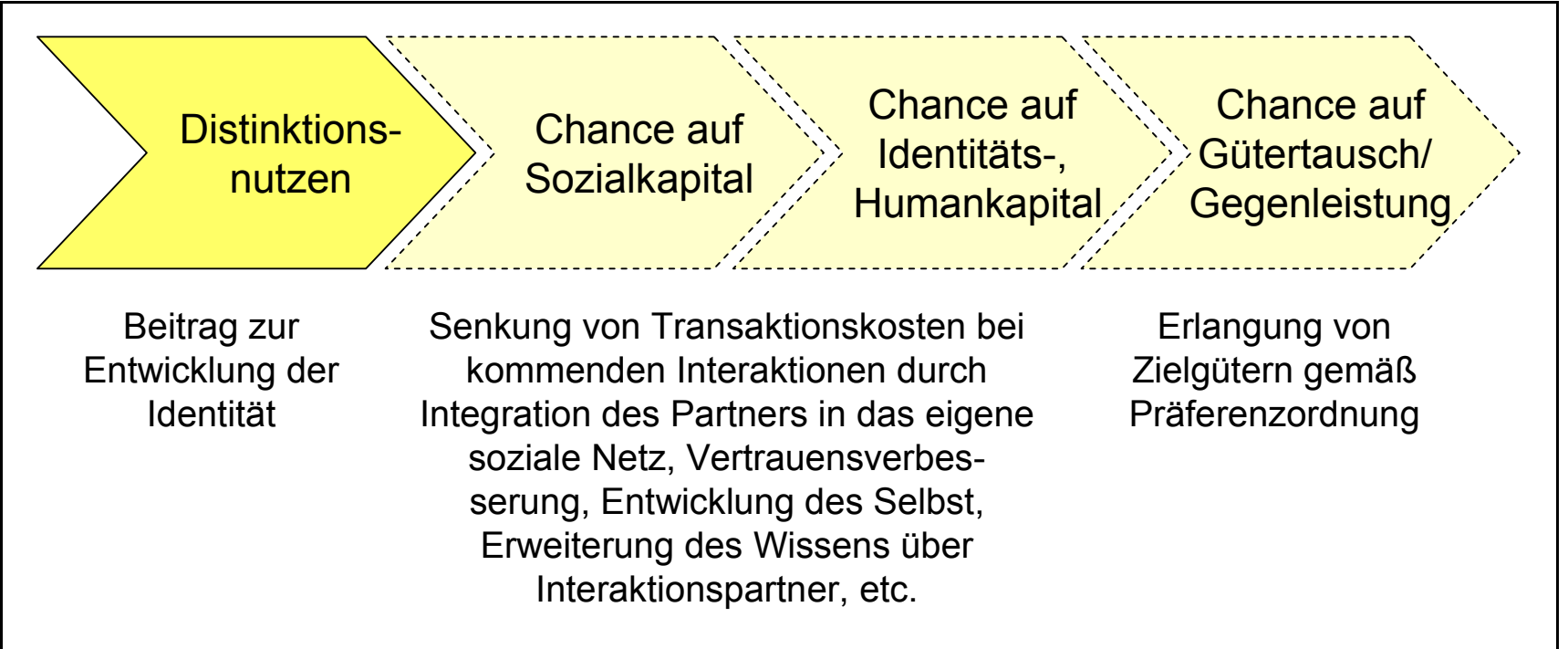

Abb. 1.7.: Anreizstruktur für ein Nutzen maximierendes Modellindividuum, soziale Interaktionen einzugehen.

6. Identitätskapital bezeichnet die in Unterscheidungen bzw. Interaktionen erworbenen Eigenschaften und Merkmale des Individuums. Die Summe der Merkmale und Eigenschaften kann als Identität des Individuums angesehen werden. Das Identitätskapital ist eng mit dem Humankapital (erworbene Eigenschaften in Form von Wissen und Informationen über die Welt und andere Individuen) und Sozialkapital (spezielle Verbindung bzw. Verbundenheit zu anderen Individuen) verwoben, wird jedoch hier isoliert betrachtet, um die besondere Bedeutung der Identität und der Identitätserhaltung zu verdeutlichen. Es kann als eigene Kapitalart in die erweiterte Kapitaltheorie integriert werden (Abb. 1.8.). Identitätskapital liefert so einen Hinweis darauf, warum sich komplexe soziale Interaktionen entwickeln. Je komplexere Begriffe in der Umwelt existieren (genauer: von der Umwelt unterschieden sind), desto mehr verschiedene Merkmale kann ein Individuum seinem 
Identitätskapital in Interaktionen hinzufügen. Das Zusammenfügen von einzelnen Unterscheidungen $\mathrm{zu}$ Zeitreihen oder die Zuordnung zu verschiedenen Individuen erhöht die Merkmalsvielfalt exponentiell.

Klassische Kapitaltheorie

Erweiterte Kapitaltheorie zur Integration von zentralen Lebensbereichen wie Ehe, Bildung, sozialem Verhalten

\section{Erweiterung}

zur Integration der Anforderungen an ein Individuum und die Identität in der Spät-/ Postmoderne

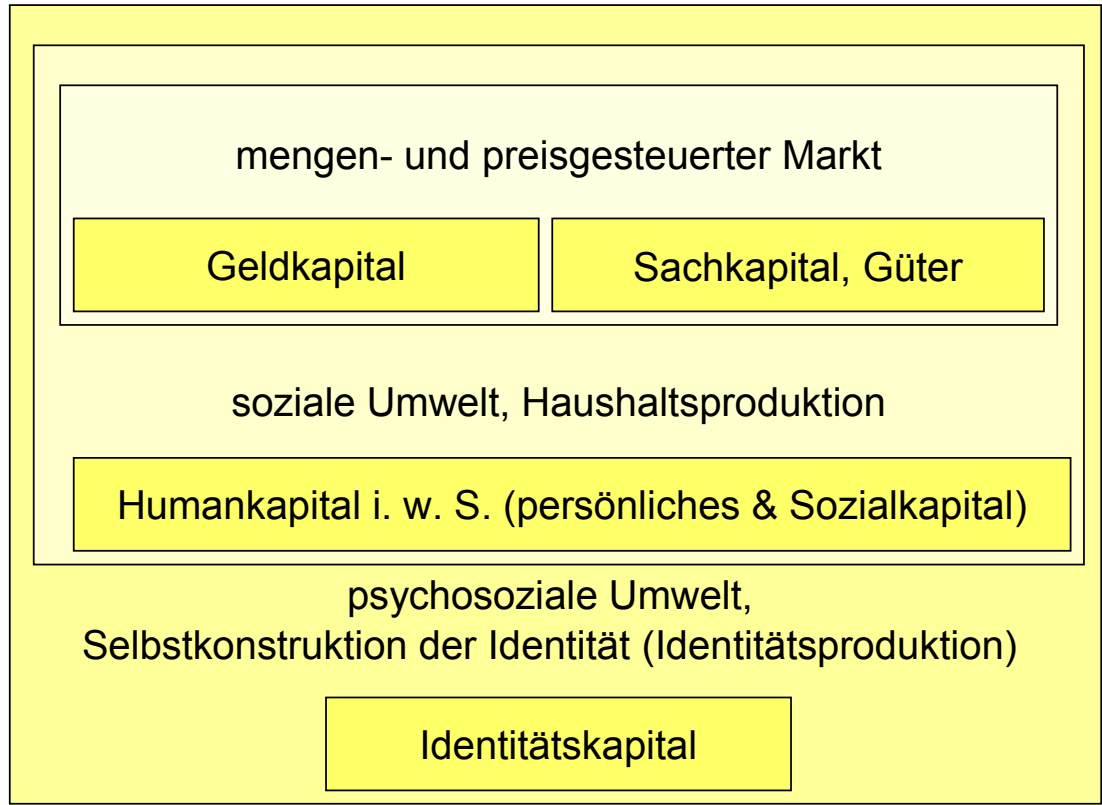

Abb. 1.8.: Schema der schrittweisen Erweiterung der Kapitaltheorie und ihrer Erklärungsreichweite.

7. Somit ergibt sich, dass der ökonomische Nutzen der einem Individuum aus einer sozialen Interaktion entsteht, aus drei Bausteinen besteht. Aus der Interaktion entsteht (1) unmittelbar der direkt wirksame Distinktionsnutzen. Zusätzlich wirkt die soziale Interaktion positiv auf die Kapitalbestände des Individuums, nämlich (2) durch die Bildung von Identitätskapital, und (3) wirkt die Vermehrung des Identitätskapitals auf die Bestände des Humankapitals. Systematisch lassen sich 2 Bereiche identifizieren, in denen der Nutzesoldo eines Individuums für eine soziale Interaktion positiv ist. Dies ist einmal ein Bereich sehr geringer Investitionen, etwa der Gruß oder eine kleine freundliche Unterhaltung, hier überkompensiert der Distinktions- und Identitätskapitalnutzen die Investitionen in Form z. B. der aufgewendeten Zeit. Steigen die Kosten an, sinkt der Nutzensoldo, bis die Bildung von Humankapital aus dem zusätzlichen Identitätskapital zusätzlichen Nutzen generiert und so einen 2. Bereich positiven Gesamtnutzens einer Interaktion erzeugt, der mit erheblich höheren individuellen Investitionen, z.B. einer Einladung zu einem gemeinsamen Essen oder Theaterbesuch, verbunden ist, bei dem man sich intensiver kennen lernt und darüber Identitäts-, persönliches und Sozialkapital vermehrt.

8. Die Ergänzung der ökonomischen Betrachtung öffentlicher Güter mit dem Konzept des aus Identitätserwägungen abgeleiteten Distinktionsnutzens und Identitätskapitals stellt einerseits auf der Basis des Distinktionsnutzens (funktionales Modell) die Grundlage für 
die Entstehung sozialer Interaktionen und somit von Sozialstrukturen bereit. Auf diesen Sozialstrukturen beruhen die Normen, die soziale Kontrolle, die zur Entstehung öffentlicher Güter nötig sind. Andererseits wird die Anreizstruktur zur Beitragsleistung für ein öffentliches Gut über die Aspekte sozialer (Bildung von Sozialkapital bei gemeinsamer Erstellung des öffentlichen Gutes) und Humankapital-Anreize (Bildung von Humankapital

i. e. S., also individuellem Wissen und individuellen Fähigkeiten bei der Beitragsleistung) auf identitätsbildende Anreize erweitert. Wenn ein Individuum einen Beitrag zu einem öffentlichen Gut leistet, dann handelt es sich ökonomisch betrachtet um den Tausch einer Leistung, nicht gegen ein Nutzungsrecht am öffentlichen Gut, sondern gegen Human-, Sozial- oder Identitätskapital, das bei der öffentlichen Beitragsleistung entsteht.

\subsection{Anhang}

\subsubsection{Die monetarisierte Auszahlungsfunktion eines öffentlichen Gutes}

Als die klassische Auszahlungsfunktion eines öffentlichen Gutes wird Gleichung 1.11.1 verwendet. Für eine Periode für das Individuum (i) ergibt sich:

$$
\pi_{i}=e-c_{i}+f \cdot \frac{1}{N} \cdot \sum_{j=1}^{N} c_{j}
$$

(Gleichung 1.11.1)

Wobei $e$ die Ausstattung in Geldeinheiten und $c$ der individuelle Beitrag sind, sowie $f$ der (Zins-)Faktor (üblich $1 / N<f<1$ ) ist, mit dem die Summe aller Beiträge multipliziert wird und $N$ die Zahl der Individuen und j der Index für alle Individuen ist, besteht ein individueller Anreiz, nichts beizutragen und die Trittbrettfahreroption zu nutzen, da der Grenzertrag des „Nichts-Investierens“ mit 1 größer ist als der Grenzertrag des öffentlichen Gutes (f). Ein einzelnes individualistisches neoklassisches Modellindividuum innerhalb einer Gesellschaft verschiedener Individuen würde von Beginn an nichts geben und als Trittbrettfahrer auftreten, eine Gesellschaft aus Modellindividuum könnte das öffentliche Gut nicht erstellen oder erhalten.

Das vorgeschlagene Konzept des Identitätsnutzens erweitert die Gleichung 1.11.1 in gleicher Weise, wie die oben dargestellten Nutzenfunktionen, indem zur Verzinsung des öffentlichen Gutes $\left(f_{1}\right)$ noch der verzinste Nutzen des Identitätskapitals $\left(f_{2} * I\right)$ infolge sozialer Interaktion 
und Anerkennung bzw. Merkmalszuschreibung tritt. Nun stehen der Investition $c_{\mathrm{i}}$ die Verzinsung aller Nutzenanteile plus der verzinste Distinktions- und Identitätsnutzen $I$ gegenüber.

Die Schwelle, die einen Beitrag rentabler macht, wird gesenkt.

$$
\pi_{i}=e-c_{i}+f_{1} * \frac{1}{N} * \sum_{j=1}^{N} n_{j}+f_{2} * I
$$

(Gleichung 1.11.2).

Analog zu den Gleichungen 1.8.1 und 1.8.5 kann diese Gleichung auch für heimliche und öffentliche egoistische Nutzer erweitert werden. Dabei ergibt sich auch in der monetarisierten Formel dann ein Anreiz für kooperatives und gegen egoistisches Verhalten bei der Nutzung öffentlicher Güter, wenn der Identitätsnutzen bzw. dessen Auszahlungsfunktion hoch genug ist.

\subsection{Literatur}

Abel, G. 1999: Sprache, Zeichen, Interpretation. Frankfurt a.M.: Suhrkamp.

Akerlof, G. A. 1970: The Market for 'Lemons': Quality Uncertainty and the Market Mechanism. Quarterly Journal of Economics, 84(3), pp. 488-500.

Akerlof, G. A., R. E. Kranton 2000: Economics and Identity. Quarterly Journal of Economics 115: 715-753.

Akerlof, G. A., R. E. Kranton 2002: Identity and Schooling: Some Lessons for the Economics of Education. Journal of Economic Literature, December 2002, v. 40, iss. 4, pp. 1167-1201.

Akerlof, G. A., R.E. Kranton 2005: Identity and the Economics of Organizations, Journal of Economic Perspectives 19 (1), 9-32.

Alchian, A. A., H. Demsetz 1972: Production, Information Costs and Economic Organisation. American Economic Review 62, pp. 777-795.

Andreoni, J. 1988: Privately Provided Public Goods in a Large Economy: The Limits of Altruism. Journal of Public Economics, v.35, February 1988, 57-73.

Andreoni, J. 1990: Impure Altruism and Donations to Public Goods: A Theory of Warm-Glow Giving. Economic Journal, v.100, June 1990, 464-477

Andreoni, J. 1995: Warm-Glow Versus Cold-Prickle: The Effects of Positive and Negative Framing on Cooperation in Experiments. The Quarterly Journal of Economics, Vol. 110, No. 1. (Feb., 1995), pp. 121.

Andreoni, J., J.H. Miller 2002: Giving according to GARP: an experimental test of the consistency of preferences for altruism. Econometrica 70 (2002) 737-753.

Arrow, K. J. 1981: Optimal and Voluntary Income Distribution. In: S. Rosefielde (Ed.): Economic Welfare and the Economics of Soviet Socialism. Cambridge: Cambridge University Press 1981 pp. 267-288

Arrow, K. J. 1985: The Economics of Agency, in: Pratt, J. W., R.J. Zeckhauer (Ed.): Principals and Agents: The Structure of Business. Boston, pp. 37-51.

Axelrod, R. 1981: The Emergence of Cooperation among Egoists. The American Political Science Review, Vol. 75, pp. 306-318.

Beck, U. 1986: Risikogesellschaft. Auf dem Weg in eine andere Moderne. Suhrkamp, Frankfurt a.M. (Engl.: Beck, U. 1992: Risc Society: Towards a New Modernity. Landon: Saga.) 
Becker, G. S. 1962: Investment in Human Capital: A Theoretical Analysis. In: Journal of Political Economy 70, pp. $9-49$

Becker, G. S. 1974: A Theory of Social Interactions. The Journal of Political Economy, 1974, 82(6), pp. 106393.

Becker, G. S. 1976: The economic Approach to Human Behaviour. London, Chicago: The University of Chicago Press.

Becker, G. S. 1976b: Altruism, Egoism, and Genetic Fitness: Economics and Sociobiology, Journal of Economic Literature 14 No.3, pp. 814-826.

Becker, G. S. 1993: Nobel Lecture: The Economic Way of Looking at Behavior. Journal of Political Economy, 1993, 101(3), pp. 385-409.

Becker, G. S. 1996 (1993): Die ökonomische Sichte menschlichen Verhaltens. In: Pies, Ingo (Hg.): Familie, Gesellschaft und Politik - die ökonomische Perspektive. Tübingen: Mohr. S. 21-49. Dt. Übersetzung von Becker 1993)

Becker, G. S. 1996: Accounting for Tastes. Cambridge, Mass.: Harvard University Press.

Becker, G. S., K. M. Murphy 1987: The Family and the State. Economics Research Center/ NORC Discussion Paper no 87-15. dt: Becker, Murphy 1996 (1987): Die Familie und der Staat. In: Pies, Ingo (HG): Familie, Gesellschaft und Politik - die ökonomische Perspektive. Tübingen: Mohr. S.197-226.

Becker, G. S., K. M. Murphy 1988: A Theory of Rational Addiction. Journal of Political Economy 96: 675-700

Becker, G.S., K. M. Murphy 1993: A Simple Theory of Advertising as a Good or Bad. Quart. J. Econ. (November 1993), pp. 941-964.

Bénabou, R., J. Tirole 2004: Incentives and Prosocial Behaviour. Discussionpapers in Economics No. 230. Princeton University. http://www.wws.princeton.edu/ econdp/pdf/dp230.pdf (7.7.06)

Blau, P. 1964: Exchange and Power in Social Life. New York: Wiley.

Boyer, M. 1978: A Habit Forming Optimal Growth Model. International Economic Review 19 (October 1978) pp. 585-609.

Boyer, M. 1983: Rational Demand and Expenditures Patterns under Habit Formation. J. Econ. Theory 31 (October 1983). pp. 27-53.

Breakwell, G. 1989: Coping with threatened identities. London: Methuen.

Brennan, G., J. M. Buchanan 1993: Die Begründung von Regeln. Konstitutionelle Politische Ökonomie. Tübingen.

Breuer, M., A. Brink, O. Schumann 2003.: Wirtschaftsethik als kritische Sozialwissenschaft. St. Galler Beiträge zur Wirtschaftsethik. Bern : Haupt.

Buchanan, J. M. 1991: Constitutional Economics. Oxford. Cambridge.

Buchanan, J. M., G. Tullock 1962: The Calculus of Consent. Logial Foundations of Constitutional Democracy. Michigan.

Camilleri, C. 1991. La constmction identitaire: essai d'une vision d'ensemble. Les Cahiers Intemationaux de Psychologie Sociale, 1991 (1/2), pp. 77-90.

Coase, R. H. 1937: The Nature of the Firm. Economia IV, pp. 386-405.

Coase, R. H. 1960: The Problem of Social Costs. Journal of Law and Economics III, pp. 1-44.

Coase, R. H. 1984: The New Institutional Economics. Journal of Institutional and Theoretical Economics 140, S. 229-231.

Coleman, J. 1988: Social Capital in the Creation of Human Capital. In: American Journal of Sociology, 94 Supplement, pp. S95-S120.

Coleman, J. 1990: Foundations of Social Theory, Cambridge, Mass.

Collard, D. A. 1978: Altruism and Economy. Oxford: Martin Robertson.

Conzen, P. 1990. Erik H. Erikson und die Folgen. Heidelberg: Asanger.

Cornes, R., T. Sandler 1999: The theory of externalities, public goods, and club goods. 2. ed., reprint. Cambridge 
u. a.: Cambridge Univ. Press.

Crook, S., J. Pakulski, M. Waters 1992: Postmodernisation: Change in Advanced Society. London: Saga.

Cyert, R. M., March, J. G. 1963: A Behavioral Theory of the Form. Englewood Cliffs, New Jersey.

Davis, D.D., C.A. Holt 1992: Experimental Economics. Princeton: University Press.

Demsetz, H. 1967: Toward a Theory of Property Rights. American Economic Review, 2, pp. 347-359.

Derrida, J. 1982: Margins of Philosophy. Brighton: Harvester.

Diekmann, A. 1993: Sozialkapital und das Kooperationsproblem in sozialen Dilemmata. In: Analyse und Kritik, $15,1, \mathrm{~S} .22-35$.

Dietz, T., Fitzgerald, A., Shwom, R. 2005. Environmental Values. Annu. Rev. Environ. Resour. 30, pp. 335372.

Durlag, S. N. 2002: On the Empirics of Social Capital. The Economic Journal 112(2002). pp. F459 - F479.

Erikson, E. H. 1965: Kindheit und Gesellschaft. Stuttgart: Klett.

Erikson, E. H. 1970: Jugend und Krise. Die Psychodynamik im sozialen Wandel. Stuttgart: Klett.

Erikson, E. H. 1973: Identität und Lebenszyklus. Frankfurt/M.: Suhrkamp.

Esser, H. 1993: Soziologie. Allgemeine Grundlagen. Frankfurt am Main: Campus-Verlag.

Euler, M. 2006: Soziales Kapital. Ein Brückenschlag zwischen Individuum und Gesellschaft. Oldenburg: BISVerlag.

Euler, M., Freese, J. 2007: Einführung eines Distinktionsnutzens beim „homo oeconomicus“ als Lösungsmöglichkeit für soziale Dilemmata. In prep.

Feldman, T.R., Assaf, S. 1999: Social Capital: Conceptual Frameworks and Empirical Evidence. An Annotated Bibliography, Social Capital Initiative Working Paper No. 5, The World Bank, Social Development Department, Washington DC.

Furubotn, E. G., S. Pejovich 1972: Property Rights and Economic Theory: A Survey of Recent Literature. Journal of Economic Literature, 10, pp. 1137- 1162.

Furubotn, E. G., S. Pejovich 1974: The Economics of Property Rights. Cambridge, MA.

Gardner, R., E. Ostrom, J. Walker 1990: The Nature of Common Pool Resource Problems. Rationality and Society 2:335-358.

Gibbins, J.R., B. Reimer 1999: The Politics of postmodernity. London: SAGA.

Glaeser, E.L., D. Laibson, B. Sacerdote 2002: An economic approach to social capital. The Economic Journal 112, pp. F437-F458.

Habisch, A. 1998: Extending Capital Theory - gesellschaftspolitische Implikationen eines theoretischen Forschungsprogramms. In: Pies, I.; Leschke, M. (Hg.): Gary Beckers ökonomischer Imperialismus, Tübingen 1998, S.31-50.

Hammond, P. 1975: Charity: Altruism or Cooperative Egoism? In E.S. Phelps (ed.): Altruism, Morality, and Economic Theory. New York: Russell Sage Foundation, pp. 115-131.

Hardin, G. 1968: Tragedy of the Commons. Science 162, pp. 1243-1248.

Hartmann, H. 1950: Comments on the Psychoanalytic Theory of the Ego. Study of the Child 5, pp.74-96.

Hassan, I. 1985: The Culture of Postmodernism. Theory, Culture \& Society 2, pp. 119-131.

Haug, S. 1997: Soziales Kapital. Ein kritischer Überblick über den aktuellen Forschungsstand, Mannheimer Zentrum für Europäische Sozialforschung, Arbeitspapiere Arbeitsbereich II, Nr. 15, Mannheim.

Heckathorn, D.D. 1996: The dynamics and dilemmas of collective action. American Sociological Review (61), S. 250-277.

Hermann-Pillath, C., J. J. Lies 2001: Sozialkapital. WiSt Heft 7, S. 362-366.

Holländer, H. 1990: A Social Exchange Approach to Voluntary Cooperation. American Economic Review, Vol. 80 No.5, December 1990, pp.1157-1167.

Homann, K., I. Pies, 1994: Wirtschaftsethik in der Moderne. Zur ökonomischen Theorie der Moral, in: Ethik und Sozialwissenschaften, Heft 1, S. 3-12. 
Inglehart, R., C. Welzel 2005: Modernization, Cultural Change and Democracy. Cambridge u.a.: Cambridge Press.

Jameson, F. 1983: Postmodernism and the consumer society. In: N. Foster (Ed.): Postmodern Culture. Seattle: Bay View.

Jameson, F. 1984: Postmodernism, or The Cultural Logic of Late Capitalism. New Left Review 146, pp.53-92.

Jans, M. 2003: Sozialkapitalkonzepte und ihre Brauchbarkeit in der Personal- und Organisationsforschung. Diskussionsbeiträge aus dem Fachbereich Wirtschaftswissenschaften der Universität Duisburg-Essen, Nr. 128, Oktober 2003. Essen. http://www.uni-essen.de/personal/Sozialkapital.pdf (12.10.06).

Jensen, M. C., W.H. Meckling 1976: Theory of the Firm: Managerial Behavior, Agency Costs and Ownership Structure. Journal of Financial Economics, 3, pp. 305-360.

Kahneman, D., A. Tversky 1979: Prospect Theory: An Analysis of Decision under Risk. Econometrica 47(2), pp. 263-291.

Kahneman, D., A. Tversky 1984: Choices, Values, and Frames. American Psychologist 39, pp. 341-350.

Kellner, D. 1992: Popular culture and the construction of postmodern identities. In S. Lash \& J. Friedman (Eds.): Modernity \& identity (). Oxford UK: Blackwell. pp 141-177.

Keupp, H., T. Ahbe, W. Gmür, R. Höfer, B. Mitzscherlich, W. Kraus, F. Straus 2006: Identitätskonstruktionen. Das Patchwork der Identitäten in der Spätmoderne. Reinbek: Rowohlt. 3. Auflage.

Kirchgässner, G. 1991: Homo oeconomicus. Das ökonomische Modell individuellen Verhaltens und seine Anwendung in den Wirtschafts- und Sozialwissenschaften. Tübingen.

Krappmann, L. 1988: Soziologische Dimensionen der Identität. Strukturelle Bedingungen für die Teilnahme an Interaktionsprozessen. 7. Auflage. Stuttgart: Klett. 1. Aufl.

Kraus, W. 2000: Das erzählte Selbst: die narrative Konstruktion von Identität in der Spätmodeme. Herbolzheim: Centaurus-Verl.-Ges. 2. Auf.

Kuhn, M.H. 1964: Self. In Gould/ Kolb (Eds.) A dictionary of social science. London: Tavistock. Pp.628-630.

Kurz, M .1977: Altruistic Equilibrium. In: B. Balassa, R. Nelson (eds): Economic Progress, Private Values and Public Policy. Amsterdam: North Holland.

Laffont, J.-J. 1975: Macroeconomic constraints, economic efficiency and ethics: An introduction to Kantian economics. Economica, vol. 42, pp.430-437.

Lash, S., J. Friedman (Eds.) 1992. Modernity \& identity. Oxford UK: Blackwell.

Lash, S., J. Urry 1994: Economics of Signs and Space. London: Saga.

Ledyard, J.O. 1995: Public Goods: A Survey of Experimental Research. In: J. Kagel, A.E. Roth (Eds.): Handbook of Experimental Economics. Princeton: University Press.

Lyotard, J.-F. 1994: Das postmoderne Wissen. Ein Bericht. Wien: Edition Passagen.

Lee, D. R., R. B. McKenzie 1987: Regulating Government. A Preface to Constitutional Economics. Toronto.

Lindenberg, S. 1985: An Assessment of the New Political Economy for the Social Sciences and for Sociology in Particular. In: Sociological Theory 3: 99-114

Lyon, D 1994: Postmodernity. Buckingham: Open Universuty Press.

Lyotard, J.-F. 1984: The Postmodern Condition: A Report on Knowledge. Manchester: Manchester University Press.

Manstetten, R. 2002: Das Menschenbild der Ökonomie. Freiburg.

March, J. G., H.A. Simon 1958: Organisations. New York.

Marcia, J. E. 1993: The status of the statuses: Research review. In J. E. Marcia, A. S. Waterman, D. R. Matteson, S. L. Archer \& J. L. Orlofsky (eds.): Ego identity. A handbook for psychosocial research. New York: Springer, pp. 22-41.

Marggraf, R., S. Streb 1997: Ökonomische Bewertung der natürlichen Umwelt, Heidelberg.

Margolis, H. 1982: Selfishness, Altruisms and Rationality. A theory of Social Choice. Cambridge: Cambridge University Press.

Markus, H. R. 1977: Self-schemata and processing information about the self. Journal of Personality and Social 
Psychology, 35, pp. 63-78.

McCall, G.J., J.L. Simmons 1966: Identities and Interactions. An Examination of Human Associations in Everyday Life. New York: Free Press.

Mead, G. H. 1968 (1973): Geist, Identität und Gesellschaft. Aus der Sicht des Sozialbehaviorismus. Frankfurt/M.: Suhrkamp. (Engl. 1934: Mind, Self and Society. From the Standpoint of a social behaviourist. Chicago.)

Meckling, W.H., 1976: Values and the Choice of the Individual in the Social Sciences. In: Schweizerische Zeitschrift für Volkswirtschaft und Statistik 112, pp.545-560

Meyer-Faje, A., P. Ulrich (Hrsg.) 1991: Der andere Adam Smith - Beiträge zur Neubestimmung von Ökonomie als Politischer Ökonomie. St. Galler Beiträge zur Wirtschaftsethik, Bd. 5, Bern / Stuttgart.

Mueller, D. C. 1986: Rational Egoism Versus Adaptive Egoism as Fundamental Postulate for a Descriptive Theory of Human Behavior. Public Choice 51, pp. 3-23.

North, D. C. 1988: Theorie des institutionellen Wandels. Eine neue Sicht der Wirtschaftsgeschichte. Tübingen.

North, D. C. 1990: Institutions, Institutional Change and Economic Performance. Cambridge.

North, D. C. 2002: Institutions, institutional change and economic performance. Cambridge: CUP.

Oerter, R., L. Montada 1998: Entwicklungspsychologie. 4.Aufl. Weinheim: PSU.

Olson, M. 1965: The Logic of Collective Action, New Haven/London.

(dt.: Die Logik kollektiven Handelns. Kollektive Güter und die Theorie der Gruppen, Tübingen 1968).

Ostrom, E., J. Burger, C. B. Field, R. B. Norgaard, D. Policansky 1999: Revisiting the Commons: Local Lessons, Global Challenges. Science 284, pp. 278-282.

Ostrom, E., R. Gardner, J. Walker, 1994: Rules, Games, and Common-Pool Resources. Ann Arbor: University of Michigan Press.

Pareto, V. 1980: Compendium of General Sociology. Minneapolis: University of Minnesota Press.

Parsons, T. 1976: Zur Theorie sozialer Systeme. Opladen: Westdt. Verlag.

Pies, I. 1998: Theoretische Grundlagen demokratischer Wirtschafts- und Gesellschaftspolitik - Der Beitrag Gary Beckers. In: . In: Pies, I.; Leschke, M. (Hg.): Gary Beckers ökonomischer Imperialismus, Tübingen 1998, S.1-29.

Pigou, A. C. 1920: The Economics of Welfare. London: Macmillan.

Quine, W.V.O. 1960: Word and Object, Cambridge/ Mass: MIT Press. Dt: Quine 1969: Grundzüge der Logik. Frankfurt: Suhrkamp.

Rawls, J. 1971: Theory of Justice. Cambridge, Mass. Dt: Eine Theorie der Gerechtigkeit, Frankfurt a. M. 1975.

Richter, R., E.G. Furubotn 2003: Neue Institutionenökonomik. Tübingen.

Rokeach, M. 1969: Beliefs, attitudes, and values. San Francisco.

Rorty, R. 1979: Philosophy and the Mirror of Nature. Oxford: Blackwell.

Rorty, R. 1989: Contingency, Irony and Solidarity. Cambridge: Cambridge University Press.

Rose, A. M .1962a: Preface. In: Rose, A.M. (ed.): Human Behavior and Social Processes. London: Routledge \& Kegan Paul. pp.VII-XII,

Rose, A. M. 1962b: Systematic Summary of Symbolic Interaction Theory. In: Rose, A.M. (ed.): Human Behavior and Social Processes. London: Routledge \& Kegan Paul. pp.3-19

Rose, M. 1991: The Post-Modern and the Post-Industrial. Cambridge: Cambridge University Press.

Rosenau, P.M. 1992: Post-Modernism and the Social Science. Princeton: Princeton University Press.

Ryder, H. E. Jr., G. M. Heal 1973: Optimal Growth with intertemporally Dependent Preferences. Review of Economic Studies 40(1), pp. 1-31.

Samuelson, P.A. 1969: The pure theory of public expenditure and taxation. In: J. Margolis and H. Guitton (eds.): Public Economics, London: Macmillan, pp. 98-123

Sandler, T. 2001: On Financing Global and International Public Goods; World Bank Policy Research Working 
Paper No. 2638. http://ssrn.com/abstract $=632706$

Schlösser, H. J. 1992: Das Menschenbild in der Ökonomie: Die Problematik von Menschenbildern in den Sozialwissenschaften - dargestellt am Beispiel des homo oeconomicus in der Konsumtheorie. Köln: Bachem.

Schotter, A. 1981: The economic theory of social institutions. Cambridge: Cambridge Univ. Press.

Schultz, P. W. 2002: Inclusion with nature: Understanding the psychology of human-nature interactions. In: P. Schmuck, \& P. W. Schultz, The psychology of sustainable development. New York: Kluwer.

Schultz, T. W. 1961: Investment in Human Capital. The American Economic Review 1(2), pp. 1-17.

Schultz, T. W. 1973: The Value of Children: An Economic Perspective. The Journal of Political Economy, Vol. 81, No. 2, Part 2, pp. S2-S13.

Schultz, T. W. 1980: Nobel Lecture: The Economics of Being Poor. Journal of Political Economy, University of Chicago Press, vol. 88(4), pp. 639-651.

Selten, R., A. Klopstech 1984: Formale Konzepte eingeschränkt rationalen Verhaltens. In: H. Todt (Hg.): Normgeleitetes Verhalten in den Sozialwissenschaften. Berlin, S.11-34.

Sen, A.K. 1977: Rational fools: a critique of the behavioural foundations of economic theory. Journal of Philosophy and Public Affairs, vol. 6, pp317-344.

Simon, H. A. 1955: Behavioral Model of Rational Choice. Quaterly Journal of Economics Vol. 69, pp. 99-118.

Smart, B. 1993: Postmodernity. London: Routledge.

Smith, A. 1926: Theorie der ethischen Gefühle. Bd. 1. Hg. v. W. Eckstein, Leipzig 1926. (engl: „,Theory of Moral Sentiments“1759).

Spencer-Brown, G. 1973: Laws of Form. Toronto, New York, London.

Stern, P. C. 2000: Towards a coherent theory of environmentally significant behaviour. Journal Social Issues 56, pp. 407-424.

Stigler, G. J., G. S. Becker 1977: De gustibus non est disputandum. American Economic Review, 67 pp. 76-90. dt: Becker 1996: Familie, Gesellschaft und Politik - die ökonomische Perspektive. Tübingen: Mohr. S.5076.

Sugden, R. 1982: On the Economics of Philanthropy. Economic Journal, June 1982, 92, pp. 341-350.

Sugden, R. 1984: Reciprocity: The Supply of Public Goods Through Voluntary Contributions. Economic Journal, December 1984, 94, pp. 772-787.

Therborn, G. 1995: European Modernity and Beyond: The Trajectory of European Societies 1945-2000. London: Saga.

Tversky, A., D. Kahneman 1974: Judgment under Uncertainty: Heuristics and Biases. Science 185 (Sept. 1974), pp. 1124-1131.

Ulrich, P. 2001: Integrative Wirtschaftsethik. Grundlagen einer lebensdienlichen Ökonomie, 3. Aufl. Bern, Stuttgart, Wien: Haupt.

Voigt, S. 2002: Institutionenökonomik. München: Fink.

Wagner, P. 1995. Soziologie der Moderne. Frankfurt/M.: Campus.

Weimann, J. 1987: Normgesteuerte ökonomische Theorien. Frankfurt /M., New York: Campus.

Wichardt, P. C. 2006: On Why and How Identity Should Influence Utility. BBL-Papiere SoSe 2006, LeopoldFranzens-Universität Innsbruck, Institut für Wirtschaftstheorie, -politik und -geschichte, Innsbruck, Austria, http://www.uibk.ac.at/economics/bbl/bbl-s06/wichardt.pdf (6.7.2006).

Williamson, O. E. 1975: Markets and Hierarchies. Analysis and Antitrust Implications. New York.

Williamson, O. E. 1985: The Economic Institutions of Capitalism. New York.

Deutsch: Williamson, O. E. 1990: Die ökonomischen Institutionen des Kapitalismus. Tübingen.

Ziehe 1986: Ny ungdom: Om ovanliga läroprocesser. Stockholm: Norstets. 


\section{Kapitel 2}

\section{Kooperativer Naturschutz in der Kulturlandschaft ${ }^{1}$}

\subsection{Die Konfliktlage zwischen Landwirtschaft und Naturschutz ${ }^{2}$}

Die „Kontrahenten“ aus Landwirtschaft und Naturschutz sind in vielfacher Hinsicht schlecht aufeinander zu sprechen. Weite Teile der Öffentlichkeit sowie des staatlichen und privaten Naturschutzes sehen in der Landwirtschaft den Hauptverursacher für die Verarmung der Landschaft, die Monotonisierung der Kulturlandschaft, die Gefährdung von Tieren, Pflanzen und deren Lebensräumen sowie der Verschmutzung von Wasser, Boden und Luft (SRU 1985; SRU 2004, Tz 225).

Agrarpolitiker und Landwirte werfen dem Umwelt- und Naturschutz eine über die Jahrzehnte immer weiter reichende Einschränkung der betrieblichen Freiräume und Entwicklungschancen vor und pochen darauf, selbst den ,richtigen“ Naturschutz zu betreiben. Auflagen würden die landwirtschaftliche Produktion verteuern und so die internationale Wettbewerbsfähigkeit der Produkte beeinträchtigten (SRU 2002a, Tz 80 ff). Neben dem finanziellen Druck macht der Landwirtschaft die Veränderung der öffentlichen Wahrnehmung zu schaffen, die - weg von der Sicherung der Lebensmittelversorgung - zunehmend mehr auf die Umwelt- und Naturschutzaspekte fokussiert. Die öffentliche Meinung ist - anders als in den 50er bis 70er Jahren - zunehmend kritisch gegenüber der Landwirtschaft eingestellt (Heinze 1992, 22; Alvensleben 2003, 32). Nicht zuletzt stehen sich mit Landwirten und Naturschützern konträre Wertvorstellungen und Überzeugungen gegenüber, so dass es neben sachlichen Vorbehalten auch emotionale Gründe für Misstrauen gibt (SRU 2002a, Tz 83). Öffentlichkeitswirksam

\footnotetext{
${ }^{1}$ Veröffentlicht als Buchbeitrag Freese J., Rüffer C. (2005): Kooperativer Naturschutz in der Kulturlandschaft. In Feindt, Newig: Partizipation, Öffentlichkeitsbeteiligung, Nachhaltigkeit. Perspektiven der politischen Ökonomie. Metropolis. S.250-271.

${ }^{2}$ Im Folgenden wird von Naturschutz in der Kulturlandschaft oder allgemeiner, Naturschutz auf landwirtschaftlich genutzten Flächen gesprochen. Damit sind verschiedene Aspekte des Naturschutzes - wildlebende Tiere, Pflanzen, ihre Lebensräume sowie Vielfalt, Eigenart und Schönheit der Landschaft - angesprochen. Aus dem Bereich des Umweltschutzes spielt dabei der Schutz von Wasser, Boden und Luft als abiotische Lebensgrundlagen eine wichtige Rolle.
} 
verschärft wird der Konflikt durch die von beiden Seiten betriebene Verbandspolitik. Die oft rüden Vorwürfe und scharf geführten Debatten mit medienwirksamen Katastrophenszenarien schaffen ein aufgeladenes Diskussionsklima, das es schwer macht, sich mit der Gegenseite zu verständigen (siehe z. B. Heinze 1992, 106-118).

Verschiedene Entwicklungen haben in letzter Zeit Bewegung in das Verhältnis zwischen Naturschutz und Landwirtschaft gebracht. Mittlerweile greift das EU-Recht stärker im Umwelt- und Naturschutzbereich der Landwirtschaft ein (Europäische Kommission, GD Landwirtschaft 2003). Das 2003 novellierte Bundesnaturschutzgesetz schwächt die landwirtschaftliche Privilegierung und legt erstmals außerhalb des landwirtschaftlichen Rechtes Regeln der guten fachlichen Praxis fest. Zusätzlich gerät die Landwirtschaft durch die Umstrukturierungen und Kürzungen der EU-Agrarförderung ${ }^{3}$ unter Druck (siehe z. B. Henning et al. 2001). Die Subventionen an die Landwirtschaft lassen sich vor WTO und Öffentlichkeit nur noch damit rechtfertigen, dass die Landwirtschaft in Europa besondere Anforderungen im Umwelt-, Natur-, Tier- und Verbraucherschutz zu erfüllen hat (Beirat BML 1998, 22 ff).

Es ist daher nicht verwunderlich, dass die Landwirtschaft zusehends ein Interesse an Fragen des Umwelt- und Naturschutzes ${ }^{4}$ entwickelt und das Thema ernster nimmt als noch in den 90er Jahren. Aber auch der Naturschutz signalisiert mit seiner neuen, von der Politik vorgegebenen Strategie des Vorrangs von freiwilligen Vereinbarungen vor ordnungsrechtlichen Maßnahmen Interesse an der Verbesserung des Verhältnisses zwischen beiden (z. B. SRU 2002a, Tz 3). Es wird erkannt, dass die Ziele des Naturschutzes nur zusammen mit den Nutzern verwirklicht werden können.

Um die gegenwärtige Situation zu verstehen, werden im Folgenden zunächst einige psychologische Hintergründe des Konfliktes aufgezeigt, bevor die Vielschichtigkeit des Partizipationsproblems von drei verschiedenen Perspektiven verdeutlicht wird. Blickwinkel 1 stellt den

\footnotetext{
${ }^{3}$ Durch die Modulation wird im Rahmen der mit den Luxemburger Beschlüssen vom Juni 2003 vereinbarten EU-Agrarreform der Bereich der ländlichen Entwicklung und somit auch des freiwilligen Vertragsnaturschutzes und der Agrarumweltprogramme finanziell besser ausgestattet und deutlich aufgewertet. Gleichzeitig werden die direkten Förderungen und Preisstützungen gekürzt. Die Cross-Compliance-Regelungen verlangen zudem von jedem Subventionsempfänger die Einhaltung von Mindeststandards unter Androhung von Kürzungen im Falle der Nichteinhaltung.

${ }^{4}$ Seit der Diskussion zur EU-Agenda 2000 und der aktuellen EU-Agrarreform nimmt das Thema einen wachsenden Raum in der landwirtschaftlichen Literatur ein (Anzahl der Artikel mit dem Stichwort Naturschutz in der Zeitschrift Landbauforschung Völkenrode und ihren Sonderheften: vor 1980: 1x, 1980-1989: 2x, 19901999: 0x, seit 2000: 9x). Anders als z. B. beim Rat für Umweltfragen, dem wissenschaftlichen Beirat für die Beratung der Regierung im Umwelt- und Naturschutz, der sich mindestens alle zwei Jahre in seinem Umweltbericht mit dem Thema Landwirtschaft und Umweltschutz auseinander setzt, hat sich z. B. der Beirat beim Bundesministerium für Verbraucherschutz und Landwirtschaft vorher nur selten mit Themen des Naturschutzes beschäftigt. Zur zögerlichen Beteiligung der Landwirtschaft am Diskurs über die ökologischen Folgen der Landnutzungspraxis siehe z. B. Heinze 1992, 44 f und 106 ff; Pongratz 1992, 194; Schur 1990.
} 
überregional auf der Ebene der nationalen Politik und Verwaltung ablaufenden Prozess des Eindringens von Öffentlichkeit und Naturschutz in die Agrarpolitik dar. Blickwinkel 2 beleuchtet die Beteiligung der Landwirte an der Gestaltung der auf sie ausgerichteten Förderprogramme. Zuletzt widmet sich Blickwinkel 3 der Partizipation der Bevölkerung bei der Zielfindung und Ausgestaltung der regionalen Landnutzung und des regionalen Naturschutzes.

Als vielversprechende Lösung für viele der Partizipationsprobleme soll schließlich das Konzept „Regionale Partnerschaft für den Naturschutz in der Kulturlandschaft“ vorgestellt werden.

\subsection{Psychologische Hintergründe des Konflikts}

Fragt man Menschen generell danach, wie sie die Natur im Hinblick auf die Toleranz gegenüber menschlichen Einwirkungen bewerten und ihnen die Möglichkeiten einer robusten, einer fragilen, einer begrenzt toleranten und einer unberechenbaren Natur zur Auswahl gibt (Ellis/ Thompson 1997), so schätzen Menschen aus dem landwirtschaftlichen Umfeld die Natur generell als robust bis begrenzt tolerant ein. Für Menschen hingegen, die sich als Umweltund Naturschützer bezeichnen, ist die Natur in aller Regeln fragil (siehe auch Abb. 2.1.).

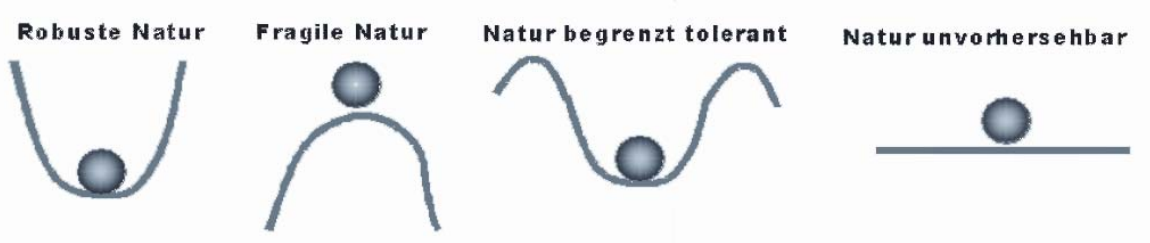

Abbildung 2.1.: Generalisierte Modellvorstellungen von der Natur. Quelle: nach Ellis \& Thompson 1997 Dieses Ergebnis erklärt sich damit, dass Landwirte in ihrer täglichen Praxis erhebliche und tief greifende Eingriffe in die Natur erleben (Pflügen eines Ackers, Flurbereinigungen, Entwässerungen) und diese Eingriffe nicht als naturgefährdend ansehen. Sie haben ein Bild von der nutzbaren Natur im Kopf, die die jahrhundertelange landwirtschaftliche Tätigkeit überstanden hat (zur Vorstellungswelt der Landwirte im Bezug auf Umwelt und Natur siehe Jurt 1998).

Mit dem Bild der Kulturlandschaft als Heimat und als Grundlage einer nachhaltigen Landwirtschaft hängt eine zweite fundamental differierende Bewertung des Naturschutzes zusammen: Fragt man nach Begründungen für Naturschutz, so ist für den Querschnitt der Bevölkerung und für Menschen aus dem landwirtschaftlichen Umfeld der Erhalt der Heimat und die Erhaltung der Nutzbarkeit des Landes der Hauptantrieb für Naturschutz. Naturschützer hingegen betonen, dass Naturschutz aufgrund wissenschaftlicher Erkenntnisse der Ökologie 
und aufgrund der moralischen Verpflichtung der Menschen gegenüber der Natur betrieben werden müsse (Reusswig 2003, siehe Abb. 2.2.).

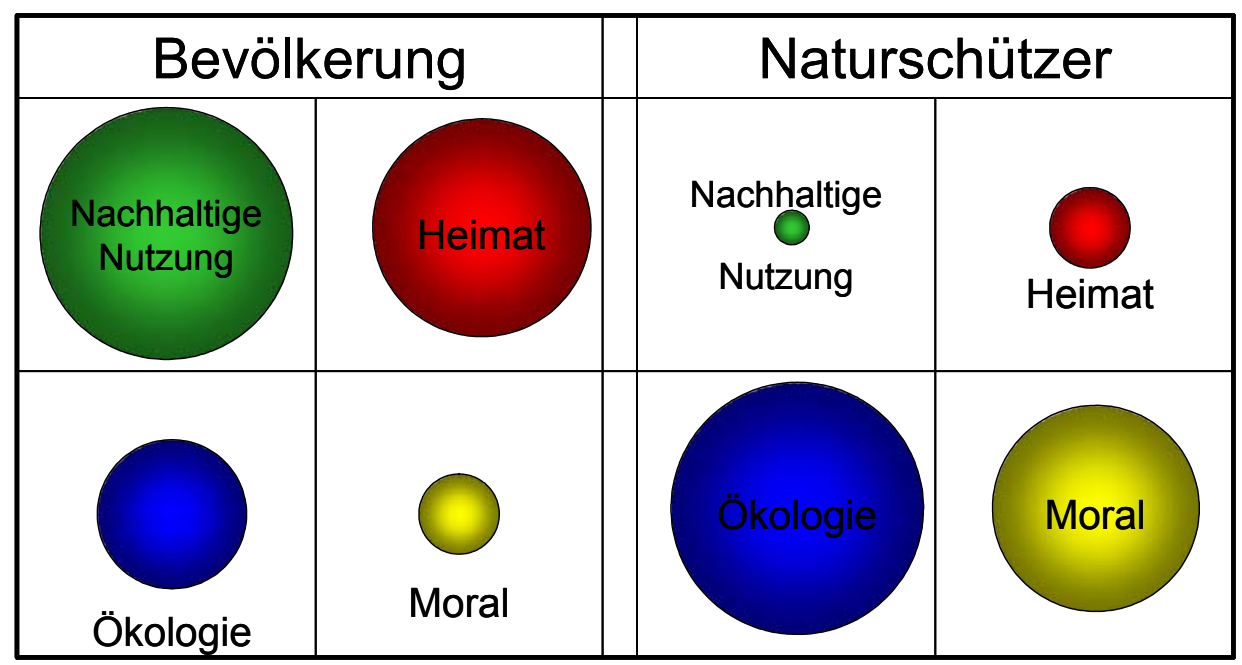

Abbildung 2.2.: Unterschiedliche Gewichtung der Gründe für Naturschutz. Quelle: Reusswig 2003.

Eine dritte wichtige Erkenntnis liegt darin, dass die regelmäßig vom Bundesumweltministerium und vom Umweltbundesamt durchgeführte Befragung „Umweltbewusstsein in Deutschland“" ein deutliches Entfernungsgefälle bei der Bewertung der Umweltqualität aufzeigt:

„Die Umwelt in der eigenen Gemeinde bzw. Stadt wird am positivsten wahrgenommen, unwesentlich schlechter beurteilt man die Umweltverhältnisse in Deutschland, aber man wechselt ins negative Lager, wenn es um die globale Umweltqualität geht ... In den Augen aller Gruppen der Bevölkerung ist lokal, hier und jetzt, eigentlich relativ wenig zu tun, es ist die weit entfernte, globale Umwelt, wo dringlich gehandelt werden muss.“" (BMU 2002, 36 f)

Eine andere Ausprägung stellt das sog. NIMBY-Phänomen (Not-in-my-backyard) dar. Niemand möchte durch Naturschutzmaßnahmen in seinen angestammten, persönlichen Freiheiten und Gewohnheiten eingeschränkt werden. Für Naturschutz und Schutzgebiete sei man schon, aber doch bitte nicht im eigenen Garten (ausführlicher siehe z. B. Ott 2002).

\subsection{Drei Blickwinkel von Partizipation}

\subsubsection{Mitgestaltung der Landnutzung durch den Naturschutz}

Unser erster Blickwinkel gilt dem Partizipationsprozess zwischen Landwirtschafts- und Naturschutzvertretern. Wie hat sich der Naturschutz Kompetenzen im Bereich der Landwirtschaft erarbeitet? Wie ist die gemeinsame Ausgestaltung der multifunktionellen Landwirtschaft (European Commission, GD Agri 1999) organisiert und wie weit reicht die Partizipation? 
Der Weg von der Lebensmittel erzeugenden zur multifunktionalen Landwirtschaft, die zusätzlich Aufgaben des Umwelt- und Naturschutzes sowie der Regionalentwicklung erfüllt, ist lang und beschwerlich. Landwirte sehen sich selbst als naturverbundene und verantwortungsbewusste Nutzer der Natur. Dies hat aber nicht dazu geführt, dass sie begeistert das Thema Naturschutz aufgegriffen hätten. Stattdessen haben sie sich vielmehr in eine Abwehrhaltung begeben und reklamieren seitdem für sich, den richtigen Naturschutz zu betreiben (Heinze 1992). Da die Landwirtschaft jedoch staatliche Zahlungen an sie durch Erbringen gesellschaftlich erwünschter Leistungen in den Bereichen Naturschutz und Regionalentwicklung legitimieren muss (siehe z. B. Beirat BML 1998a), ist die Landwirtschaft inzwischen bereit, sich das Thema auf die Fahnen zu schreiben. Zäher und langwieriger war jedoch der Prozess, den Vertretern des Politikfeldes Umwelt- und Naturschutzes Mitspracherechte in der Landwirtschaftspolitik einzuräumen.

Die Landwirtschaft ist ein traditionsreicher und wirtschaftlich aktiver Sektor, der, lange bevor der Umwelt- und Naturschutz auf der Bildfläche erschien, einen festen Platz in Öffentlichkeit, Staat, Politik und Verwaltung eingenommen und sich Zuständigkeiten, Personal und Finanzmittel gesichert hatte. ${ }^{5}$ Die Agrarpolitik war bereits lange etabliert, als der Umwelt- und Naturschutz in den 1970-er Jahren begann, sich durch bürgerschaftliches Engagement und staatliche Initiativen seinen Platz in Staat und Öffentlichkeit zu erkämpfen. Diese späte Entwicklung eines selbständigen Politikfeldes Umwelt- und Naturschutz hat zu einer verhältnismäßig schlechten Ausstattung mit Finanzmitteln, Personal und Kompetenzen geführt (SRU 2002a). Da außerdem die Zuständigkeit für die gesamte Landfläche zwischen Landwirtschaft, Forstwirtschaft, Wasserwirtschaft, Städte- und Infrastrukturbau ,aufgeteilt‘ war und dem Naturschutz nur anderweitig nicht verwertbare Flächen überlassen wurden, half man sich mit einer horizontalen Kompetenzverteilung über die bestehende Zuordnung hinweg. Umweltund Naturschutz wurde zu einer Querschnittsaufgabe erklärt ( $\$ 6$ Bundesnaturschutzgesetz) und sollte in allen anderen Politikfeldern und Sektoren berücksichtigt werden. Im Rückblick gesehen schürt die Ausgestaltung des Naturschutzes als Querschnittsaufgabe die Konfrontation. Sie wird als Einmischung in ,innere und ureigene Zuständigkeiten` angesehen (zur Entwicklung und kritischen Diskussion des staatlichen Umweltschutzes siehe z. B. Hobbensiefken 1991, 113 - 145).

Da die Zuständigkeit für den Naturschutz bis zur Einrichtung des Bundesumweltministeriums 1986 im Landwirtschaftsministerium lag, konnte die Naturschutzpolitik auf Regierungsebene

\footnotetext{
${ }^{5}$ Das Landwirtschaftsgesetz von 1955 manifestierte die Sonderstellung der Landwirtschaft gegenüber anderen Wirtschaftssektoren und schrieb die Notwendigkeit staatlicher Eingriffe und Subventionen fest (vgl. Kluge 1989 Bd.1, $227 \mathrm{ff})$.
} 
vollständig kontrolliert werden. In der Folgezeit wurde die Berücksichtigung der nun externen Naturschutzinteressen durch die Bürokratie stark gehemmt. Behörden versuchen stets, eigene Zuständigkeiten vor der Einmischung durch andere Ressorts zu schützen (zur sog. negativen Koordination siehe z. B. Scharpf 1991). Zusätzlich zur hemmenden Wirkung der administrativen Strukturen trat die Agrarlobby als starker Gestalter der Agrarumweltpolitik auf (Rudloff/ Urfei 2000, 49 f; Conrad 1987, 13 f).

Die angestrebte Integration der Umwelt- und Naturschutzpolitik in die anderen Sektoren gestaltet sich schwierig und konfliktreich und war wenig erfolgreich (Pehle 1998, 28ff; SRU 2004, Tz 1204 f). Noch bis Ende der 1990-er Jahre waren die Machtverhältnisse eindeutig. Die Landwirtschaft hatte zwar Naturschutzgesetze zu befolgen, war aber in vielerlei Hinsicht in diesen Gesetzen privilegiert. So wurden die Regeln der guten fachlichen Praxis im landwirtschaftlichen Fachrecht und nicht im Umweltschutzrecht geregelt, und die Landwirtschaft war von der Anwendung der sog. „Eingriffsregelung in Natur und Landschaft" ${ }^{\text {“6 }}$ freigestellt.

Das Vordringen naturschutzfachlicher Regelungen verlief nach einem einheitlichen Muster: Da geplante Maßnahmen in existierende Besitzstände einzugreifen drohten, wurde im politischen Prozess zunächst eine Regelung gefunden, die nicht oder kaum in den Status quo eingriff. Grenzwerte für Immissionen und Regeln für die Gute fachliche Praxis wurden so gewählt, dass die Mehrzahl der Landwirte sie einhalten konnte und es zu keiner Verhaltensänderung kam (SRU 2000, Tz. 359). Schutzgebiete wurden so abgegrenzt, dass Nutzflächen nicht betroffen waren, und Schutzgebietsverordnungen wurden so verfasst, dass sie existierende Nutzungen nicht einschränkten (z. B. SRU 2000, Tz.127, Tz.141; SRU 1996, Tz. 108).

Damit schien sich die Landwirtschaft zunächst aufgrund ihrer starken Machtposition durchzusetzen (Henrichsmeyer et al. 1984, 522 f). Die Regelungen griffen nicht in die Besitzstände der Landwirte ein, und die vielfältigen vom Naturschutz durchgeführten Planungen hatten formal nur den Status von unverbindlichen Fachplänen.

Bald ergaben sich trotz laxer Naturschutzregelungen Konflikte, welche die Landwirtschaft aufhorchen ließen. Insbesondere, wenn landwirtschaftliche Betriebe ihre Flächennutzung umstellten oder den Betrieb erweitern wollten, erwiesen sich die eingeführten Regelungen doch als Einschränkungen. Eine grundlegende Abwehr gegen jegliche Naturschutzregelung griff um sich. Dabei wurde deutlich, dass die Landwirtschaft den naturwissenschaftlich ausgearbeiteten Naturschutzplanungen wenig entgegenstellen konnte und sich die formal

\footnotetext{
${ }^{6}$ Für jeden Verursacher eines Eingriffs in Natur und Landschaft gilt gemäß $\S 18-21$ des Bundesnaturschutzgesetzes das Gebot, die Eingriffsfolgen soweit wie möglich zu vermeiden oder in ihrer Wirkintensität auf die Natur zu minimieren. Für unvermeidbare Eingriffe ist eine angemessene Kompensation zu leisten.
} 
unverbindlichen Fachplanungen zu wertvollen und im politischen Diskurs überzeugenden Argumentationsgrundlagen für Naturschutzhandeln entwickelten. So manifestierte sich das Misstrauen gegenüber naturschutzfachlichen Plänen (Freese/ Steinmann 2005; Luz 1994, 203 f.; Möller 1996).

Getragen von der öffentlichen Meinung, dass der Umwelt- und Naturschutz weiter vorangetrieben werden sollte, und aufgrund langwieriger Experten- und Lobbyarbeit des Naturschutzes gelang es immer wieder, die nun bestehenden Regelungen zu verschärfen, Grenzwerte zu erhöhen und Auflagen weiterzuentwickeln. Dies führte dazu, dass sich der Umwelt- und Naturschutz den Vorwurf einhandelte, eine Salamitaktik zu verfolgen, d.h. ständig mehr Zugeständnisse von der Landwirtschaft zu verlangen.

Aktuell werden Umwelt- und Naturschutzbelangen durch die 2005 implementierte CrossCompliance-Regelung in die landwirtschaftliche Praxis eingeführt. Durch Cross Compliance wird die EU-Förderung eines Betriebes an die Einhaltung von Standards im Umwelt-, Naturund Tierschutz gekoppelt (z. B. BMVEL 2005, 73 ff.). Diese Neuerung wurde kontrovers diskutiert, ist gegenwärtig allerdings noch nicht in ihren Auswirkungen auf Natur und Landwirtschaft bewertbar. Allerdings wird erstmals die Subventionierung der Landwirtschaft mit Umweltaspekten verknüpft und so der Umwelt- und Naturschutz weiter in das landwirtschaftliche Blickfeld gerückt.

\subsubsection{Beteiligung der Landwirte an der Ausgestaltung des Naturschutzes}

Als die politische Durchsetzung der Querschnittsaufgabe durch Berücksichtigung in den verschiedenen Fachrechten zu greifen begann, eröffnete sich ein zweites Partizipationsfeld. Ordnungsrechtliche Regelungen des Umwelt- und Naturschutzes greifen - genauso wie naturschutzfachlich motiviertes Fachrecht - in Besitzstände und die tägliche Praxis von Landnutzern ein. Der Ansatz der anreizorientierten Umsetzung des Umwelt- und Naturschutzes will Verhaltensweisen der Landnutzer beeinflussen. Daher soll der zweite Blickwinkel die Beteiligung der Landwirte an der Ausgestaltung der Naturschutzpolitik und der Entwicklung von Naturschutzregelungen beleuchten.

Der Naturschutz will das Verhalten der Landwirte über Planungen, Ordnungsrecht oder Anreize und Förderprämien umweltfreundlicher gestalten. Landwirte und ihre Vertreter achten darauf, dass ihre Betriebe rentabel bleiben, Auflagen die Produktion nicht einschränken und für Einschränkungen Ausgleich geleistet wird oder zumindest Übergangsfristen und -hilfen gewährt werden. 
Lässt sich der Naturschutz bei der Gestaltung der rechtlichen Regelungen und Maßnahmen nur von seiner naturwissenschaftlichen und ethischen Grundausrichtung leiten und kommen ökonomische und betriebliche Belange zu kurz, hat dies fatale Folgen für die Maßnahmenakzeptanz und -umsetzung. Anreizmechanismen werden ausgehebelt, da sich Landwirte nicht auf die Förderprogramme einlassen und ordnungsrechtliche Regelungen solange wie möglich ignorieren, so dass diese nur mit erheblichem Kontrollaufwand vom Naturschutz durchgesetzt werden können.

Naturschutzregelungen werden von den Fachverwaltungen (zum Verwaltungshandeln siehe z. B. Gawel 1995, Henrichsmeyer/ Witzke 1994, 470-476) ausgearbeitet. Formal gibt es für die Formulierung von Naturschutzregelungen Beteiligungsverfahren der landwirtschaftlichen Ressorts und Verwaltungen. Dies sichert jedoch nicht die Berücksichtigung der Belange der Landwirte oder die Prüfung auf betriebliche Praxistauglichkeit (Robert-Bosch Stiftung 2000, 11f).

Die Anforderungen an die Gestaltung einer Verordnung oder einer Maßnahmenrichtlinie sind enorm. Interessen und Ziele der regierenden politischen Partei, existierende gesetzliche Bestimmungen und Interessen anderer Ministerien sind zu berücksichtigen. Zusätzlich zu diesen fachlich und inhaltlich ausgerichteten Interessen müssen auch finanz- und verwaltungstechnische Rahmenbedingungen beachtet werden. Letztlich ist die Verwaltung oft der entscheidende Gestalter, insbesondere, wenn ihr die Umsetzung der Regelungen übertragen werden soll. Wie aufwändig und umfangreich darf die verwaltungsseitige Arbeit sein? Wie groß sind die Kontrollkapazitäten? Wo kann die neue Regelung oder Maßnahme in die Verwaltung eingegliedert werden?

Die Regelung wird aufgrund dieser Anforderungen und der spezifischen Arbeitsweise von Verwaltungen - ihnen sind die Einwände und Erfordernisse anderer Verwaltungen näher als Anmerkungen aus der Praxis - so gesteuert, dass sie verwaltungsfreundlich und arbeitsextensiv umzusetzen ist. Im Ergebnis werden stärker die Belange der Verwaltungen berücksichtigt, als dass die Adressaten, also die Landwirte, Gehör finden.

Im politischen Prozess auf Landes- und Bundesebene werden üblicherweise nur gebündelte Interessen, z. B. von Lobbygruppen oder von Trägern öffentlicher Belange ${ }^{7}$ berücksichtigt. In diesem Bereich der Politik geht es für Interessengruppen um strategische Ziele. Man agiert im politischen Raum und passt sich den Gegebenheiten dort an. Ein Landes- oder Bundesverband konzentriert demnach seine Arbeitskraft darauf, wegweisende und zentrale Verbandsziele mit

\footnotetext{
${ }^{7}$ Träger öffentlicher Belange bezeichnet die Vertreter von Einrichtungen, Verbänden, Lobbygruppen, etc. denen es gesetzlich erlaubt ist, zu bestimmten Vorhaben Stellungnahmen abzugeben.
} 
der Politik zu thematisieren oder zentrale Probleme der eigenen Klientel bei konkreten Politikvorhaben zu entschärfen. So es auf diesem Weg häufig nicht, umsetzungs- und praxisrelevante Gestaltungshinweise zu geben oder eine antragstellerfreundliche Gestaltung zu erwirken (zur Problematik von Lobbyverbänden siehe z. B. Henrichsmeyer/ Witzke 1994, 489-503). Ein gutes Beispiel ist die Ausgestaltung der modulationsgeförderten Agrarumweltmaßnahmen 2002 in Niedersachsen. Da die Vertreter der Landwirte gegen das Prinzip der Modulation, also die Umwandlung direkter Subventionen in Zahlungen für Maßnahmen des Umwelt-, Natur- und Tierschutzbereichs waren, haben sie politisch lange gegen die geplanten Maßnahmen gearbeitet und haben sich erst spät in der konkreten aber für die Praxis der Berufskollegen relevanten Ausgestaltung der Förderrichtlinie engagiert.

\subsubsection{Bevölkerungsbeteiligung}

Schließlich soll noch ein kurzer Blick auf eine dritte Art der Partizipation, die Beteiligung der Bevölkerung an der Ausgestaltung des regionalen Naturschutzes, geworfen werden.

Bei all den Aktivitäten, die Naturschützer und Landwirte im Bereich Naturschutz in der Kulturlandschaft entwickeln, stellt sich die Frage, wie die Bevölkerung in Entscheidungen und die Ausgestaltung eingebunden werden kann - nicht nur im Rahmen von Demokratieerwägungen ${ }^{8}$, sondern insbesondere auch, weil meist öffentliche Gelder der SteuerzahlerInnen aufgewendet werden,.

Da sich Naturschutzmaßnahmen gegenwärtig nicht mehr im Bereich der direkten Gefahrenabwehr bewegen, sondern durch die Regelungen der vergangenen Jahrzehnte bereits ein Basisschutz der Umweltgüter gewährleistet ist, erlangt die Einbindung der Bevölkerung in die Entscheidungsfindung zur Festlegung der erwünschten Schutzhöhe immer größere Bedeutung. Die Frage, wohin die begrenzten Finanzmittel fließen, ob damit z. B. eine neue Technologie der Gülleausbringung finanziert oder eine Aufwertung der Landschaft durch extensives Grünland, Streuobstwiesen, Hecken oder Alleen durchgeführt werden soll, ist eine Frage, bei der zu prüfen ist, ob die lokale Bevölkerung dies entscheiden kann (vgl. dazu den Text der Aarhus-Konvention ${ }^{9}$ ).

Im Rahmen des Forschungsprojekt „Ergebnisorientierte Honorierung ökologischer Leistun-

\footnotetext{
${ }^{8}$ „Alle Staatsgewalt geht vom Volke aus. Sie wird vom Volke in Wahlen und Abstimmungen [...] ausgeübt.“ (Art. 20 Abs. 2 Grundgesetz).

${ }^{9}$ Das ,Übereinkommen über den Zugang zu Informationen, die Öffentlichkeitsbeteiligung an Entscheidungsverfahren und den Zugang zu Gerichten in Umweltangelegenheiten“ wurde im Juni 1998 in der dänischen Stadt Aarhus unterzeichnet.
} 
gen der Landwirtschaft ${ }^{\text {“10 }}$ wurde hierzu eine Untersuchung durchgeführt, bei der 1500 Haushalte aus der Projektregion (Landkreis Northeim, Südniedersachsen) per repräsentativer Stichprobe aus dem Einwohnermelderegister angeschrieben wurden, um über die Aufteilung eines Budgets auf verschiedene Naturschutzmaßnahmen im Bereich der Kulturlandschaft des Landkreises Northeim (Neuanlage von Hecken, Erhalt extensiv genutzten Grünlandes, Förderung der Ackerbegleitflora, etc.) mit zu entscheiden. Es wurde eine 3-stufige statedpreference Befragung ${ }^{11}$ durchgeführt:

1. Güterkategorienranking für Grünland, Acker, krautige Zwischenstrukturen und Gehölze;

2. Gruppierung von 16 ökologischen Gütern ${ }^{12}$ in vier Gruppen à vier Gütern gemäß subjektiver Förderwürdigkeit;

3. Budgetspiel, in dem die Befragten aufgefordert wurden, ein fiktives Budget von $100000 €$ auf die von ihnen gebildeten Gütergruppen aufzuteilen.

Von 274 beantworteten Fragebögen gaben zunächst 86,5\% der Befragten an, dass es wichtig ist, das die Bevölkerung mitentscheiden kann. 218 (d.h. 80,4\%) signalisierten zusätzlich ihre Bereitschaft, 1-2 mal pro Jahr dabei selbst aktiv mitzuwirken. Nach der Simulation der realen Entscheidung allerdings gaben knapp über 70\% der befragten Bürger an, dass sie das Gefühl hätten, für die Beantwortung der Frage zu wenig zu wissen. Mit anderen Worten, sie fühlten sich ,subjektiv inkompetent ${ }^{\star 13}$, eine Mittelverteilung vorzuschlagen. 210 der Befragten waren am Ende dafür, die Entscheidung an ein Expertengremium (den sog. Regionalen Beirat ${ }^{14}$ ) zu übertragen. 57 Befragte sprachen sich dafür aus, dass die Entscheidung allein von der Bevölkerung getragen werden sollte, und nur 4 Personen gaben an, dass die Ministerien von Bundeslandebene oder EU, die derzeit darüber entscheiden, die Entscheidung weiterhin allein treffen sollten.

\footnotetext{
${ }^{10}$ Das Projekt ist Teil des Gesamtprojekts BIOPLEX (Biodiversität und räumliche Komplexität in Agrarlandschaften), das in Kooperation des Forschungs- und Studienzentrums Landwirtschaft \& Umwelt der Universitäten Göttingen und der Universität Gießen durchgeführt wird. Im Rahmen des Förderprogramms „Biodiversität und Globaler Wandel (BIOLOG)“ wird das Gesamtprojekt seit dem Jahr 2000 vom BMBF finanziert.

${ }^{11} \mathrm{Zu}$ einer ausführlichen Darstellung über die Technik zur ökonomischen Bewertung von Nicht-Markt-Gütern mittels Stated-preference Methoden siehe z. B. Bateman, et al. (2002).

${ }^{12}$ Je höher die römische Nummerierung, desto hochwertiger ist das Gut aus naturschutzfachlicher Sicht. Für die detaillierte Definition der Güter siehe Bertke (2005).

${ }^{13}$ Subjektiv gefühlte Inkompetenz meint, dass, außer ihrer eigenen Vorbehalte gegenüber ihrer Entscheidungskraft in dieser Sache, nichts für ein schlechteres Ergebnis gegenüber einem Entscheidungsprozess auf zentraler Regierungsebene ohne die Einbindung der betroffenen Bevölkerung spricht.

${ }^{14}$ Zum Regionalen Beirat vgl. Bertke, Hespelt, Marggraf (2005).
} 


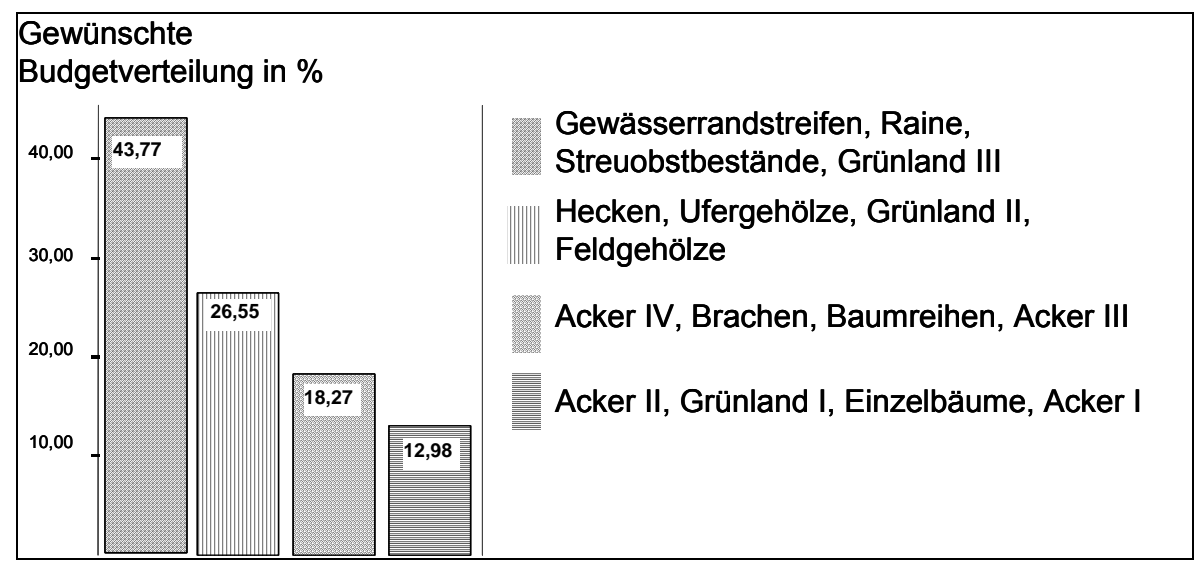

Abbildung 3: Verteilung des Budgets auf die verschiedenen Gütergruppen durch die Bevölkerung.

Der Zweifel an der eigenen Entscheidung, was fördernswert sei, deutet darauf hin, dass die Durchdringung des Umwelt- und Naturschutzes mit komplexen ökologischen Entscheidungsbegründungen dazu geführt hat, dass sich viele BürgerInnen nicht mehr trauen, eigene Zielvorstellungen für den Umwelt- und Naturschutz in ihrer Lebensumwelt zu formulieren. Dies ist beunruhigend, da der Umwelt- und Naturschutz neben den ökologischen und fachwissenschaftlichen Erfordernissen sehr von der Wertschätzung und Bewertung der Bevölkerung getragen wird ${ }^{15}$. Partizipation der Bevölkerung hat insbesondere drei Funktionen:

1. eine Lenkungsfunktion zur effizienten Allokation der Steuergelder;

2. eine Informationsfunktion ${ }^{16}$, die zur Nachhaltigkeit der Politik beiträgt;

3. eine Legitimationsfunktion für den politischen Eingriff.

Außerdem setzt sich zusehends die Erkenntnis durch, dass Umwelt- und Naturschutz neben dem Schutz von Wasser, Boden, Luft, Tieren, Pflanzen und Lebensräumen auch dem Menschen zugute kommen soll und muss. Das Konzept der Nachhaltigkeit fügt dem ökologischen Aspekt noch den sozialen und ökonomischen hinzu (siehe z. B. SRU 2002b, Tz. 30 ff). Nicht die Wissenschaft legt fest, welche Grenzwerte wir bei Gewässerverschmutzung anstreben müssen, welche Tierarten besonderen Schutz genießen sollen oder wann eine Pflanzenart als besonders gefährdet gilt und daher besonderer Schutzanstrengungen bedarf. Dies sind Entscheidungen, die im gesellschaftlichen und politischen Raum getroffen werden müssen.

\footnotetext{
${ }^{15}$ Der Naturschutz selbst nutzt, neben ökologischen und instrumentellen Naturschutzbegründungen, moralische und eudaimonische Begründungen - also solche des guten und ästhetisch erfüllten menschlichen Lebens (SRU 2002a, 17). Dennoch zeigt auch der Bericht des Sachverständigenrates zur Stärkung und Neuorientierung des Naturschutzes (SRU 2002a), dass in Naturschutzfachkreisen die Bevölkerungsbeteiligung eine relativ geringe Bedeutung besitzt. Die Beteiligungsstrategien (Mediation, Agenda-Prozess, Bürgergutachten, u. a.) spielen fast keine Rolle. Bürger treten lediglich im Bereich von Akzeptanzproblemen stärker ins Rampenlicht des Naturschutzes.

${ }^{16}$ Z. B. gefordert in Artikel 13a (Public Education and Awareness) in der Konvention über Biologische Vielfalt und in Artikel 4 und 5 der Aarhus-Konvention.
} 
Natürlich liefern die Wissenschaften wichtige Erkenntnisse, decken Zusammenhänge auf und stellen Daten zur Verfügung. Aber die Entscheidungen werden letzten Endes auf der Grundlage gesellschaftlicher und politischer Abstimmungsprozesse formuliert. Deswegen sollte dem Bürger, über die Wahl politischer Vertreter hinaus, eine Mitwirkungsmöglichkeit gewährt werden, um Entscheidungen aus Politik, Verwaltung und Wissenschaft über die Ausgestaltung seiner direkten Lebensumwelt besser mitgestalten zu können. Im Rahmen der Agenda 21-Prozesse (BMU 1993) wird bundesweit das sogenannte integrierte Steuerungsmodell „Umwelt und Entwicklung“ erprobt, das u. a. die Zielformulierung auf einer breiten Basis, die Partizipation von Verbänden und Bürgern und das Zusammenwirken von staatlichen und privaten Akteuren unter Berücksichtigung der lokalen und regionalen Ebenen anstrebt (z. B. SRU 2000, Kap. 1). Darüber hinaus können Träger öffentlicher Belange (Verbände und andere Zusammenschlüsse) z. B. Anhörungen fordern und Einwände gegen Gesetzesvorhaben vorbringen. Diese können allerdings nur die organisationsfähigen ${ }^{17}$ Bürgermeinungen erfassen. Um zu gewährleisten, dass auch die nicht organisierbaren Bürgermeinungen beachtet werden und so ein repräsentativerer Querschnitt der Meinung der Bevölkerung abgebildet wird, müssen geeignete Institutionen geschaffen werden.

Auch wenn staatliche Stellen im Vorfeld von Entscheidungen zunehmend Experten, Verbände und BürgerInnen zur Erhebung von Sachstandsdaten und zur Klärung von Interessenlagen befragen und Bürgerforen, Arbeitskreise oder Konsenskonferenzen einberufen, in denen Bürgergutachten aufgestellt oder Kompromisse für Konflikte zwischen staatlicher Steuerung, Lobbygruppen und Bürgern in Mediationsverfahren erarbeitet werden (siehe auch z. B. Oppermann 2000), bleibt als Defizit häufig die Nicht-Erfassung von nicht organisationsfähigen Bürgermeinungen. Allein eine zufällige Auswahl, wie z. B. bei Planungszellen üblich, verhindert diese Problematik (siehe Feindt 1997). Es zeigt sich, dass es zwar viele Instrumente für Beteiligungen gibt, aber sich in Deutschland noch keine Tradition, keine weithin bekannte Methode und kein allgemein verwendetes Instrument einer regelmäßigen Bürgerbeteiligung zu bestimmten Fragestellungen, die über die allgemeine Wahlbeteiligung hinausgehen, entwickelt hat. So werden oft nur organisierbare Interessen von Verbänden oder Lobbygruppen als gebündelte Meinungen für Staat und Politik sichtbar. Deswegen spielen Modelle

\footnotetext{
${ }^{17}$ In Mancur Olsons Theorie des kollektiven Handelns wird argumentiert, dass nur spezifische Gruppen bei kollektiven Zielen (wie z. B. Naturschutz) überhaupt organisationsfähig sind. Dabei ist die Anzahl der an einem Gut Interessierten wesentlich für die Organisierbarkeit. Mit anderen Worten: Nur wenn die Gruppe klein genug ist, dass der Einzelne fühlbar von geringerem Naturschutz betroffen ist, ist ein Anreiz gegeben sich für den Naturschutz einzusetzen. Große Gruppen zeichnen sich nach Olson dadurch aus, ,[...] dass kein Mitglied fühlbar betroffen wird, wenn irgendein Mitglied zur Bereitstellung des Gutes beiträgt oder nicht beiträgt, und deshalb niemand einen Grund hat, darauf zu reagieren.“(Olson 1992, S. 49). In großen Gruppen ist es rational, sich als Trittbrettfahrer zu verhalten.
} 
der Bürgerbeteiligung in der Umweltpolitik spätestens seit der Umsetzung der ersten und zweiten Säule der Aarhus-Konvention eine wichtige Rolle. ${ }^{18}$

Um einem expertokratischen und damit von den Bürgern weit entfernten Naturschutz entgegen $\mathrm{zu}$ wirken ${ }^{19}$ und Bürgern sehr einfache Beteiligungsmöglichkeiten für den regionalen Umwelt- und Naturschutz einzuräumen, eignen sich beispielsweise Regionale Partnerschaften. In regionalen Partnerschaften wird über intensive Öffentlichkeitsarbeit die Bevölkerung informiert und über Befragungen und offene Beteiligungsverfahren werden sie dazu ermuntert, aktiv in die Gestaltung ihres Lebensumfeldes einzugreifen.

\subsection{Regionale Partnerschaft für den Naturschutz in der Agrar- und Kulturlandschaft}

In der EU hat sich die Ansicht durchgesetzt, dass es kooperativer Steuerungsansätze im Bereich des Umwelt- und Naturschutzes bedarf (SRU 2004, Tz 1209 ff). Das Prinzip der Subsidiarität, d.h. Entscheidungskompetenzen auf geeigneter dezentraler Ebene zu verankern, ist eine zentrale Leitlinie der EU. ${ }^{20}$ Im Rahmen der sogenannten Kontextsteuerung (siehe z. B. Willke 1999, 39) wird vermehrt auf die verbandliche Selbststeuerung gesetzt, so dass der Staat dort vorhandenes Know-how nutzen kann. Im Folgenden wird dafür das Konzept der Regionalen Partnerschaft „Naturschutz in der Kulturlandschaft“ vorgestellt.

Ziel einer regionalen Partnerschaft ist die regionale Anpassung der Naturschutzstrategien und -instrumente und die Akzeptanzsteigerung für den Naturschutz vor Ort. Vielerorts muss dafür eine Kommunikations- und Partizipationskultur für Aufgaben des Naturschutzes in der Kulturlandschaft etabliert werden.

Die vorhandenen Verwaltungen und Verbände haben die Probleme zwischen den Akteuren und ihren verschiedenen Sichtweisen, Zielen und Motivationen nicht lösen können. Insbesondere bestehen gegen existierende Strukturen vielfältige Vorbehalte. Naturschützer würden aufbegehren, wenn man den Landwirtschaftskammern eine zentrale Zuständigkeit für den Naturschutz in der Kulturlandschaft übertragen würde. Die Landwirte hingegen hätten Vorbehalte, Naturschutzbehörden oder Naturschutzverbänden zentrale Aufgaben zuzubilligen. Daher bietet es sich an, die Zusammenarbeit in einer neuen Form, z. B. als Projekt oder

\footnotetext{
${ }^{18}$ Die Aarhus Konvention stellt über 3 Säulen sicher, dass für Bürger der Zugang zu Umweltinformationen gewährleistet wird (1. Säule), Öffentlichkeitsbeteiligungen ausgeweitet (2. Säule) und zukünftig Bürger Klagerechte in Umweltangelegenheiten bei Gerichten erhalten (3. Säule).

${ }^{19}$ In wie weit Naturschutz der Zustimmung und Unterstützung der Bevölkerung bedarf und welche Vorteile ein breit getragener Naturschutz hat, diskutiert die Literatur zu „Akzeptanz und Akzeptanzdefiziten im Naturschutz“ (siehe z. B. Ott 2002, Stoll 1999, Beirat BMU 1995).

${ }^{20}$ Das Subsidiaritätsprinzip wurde durch die Einheitliche Europäische Akte 1987 in die europäische Umweltpolitik eingeführt und 1992 im Maastrichter Vertrag als allgemeines Prinzip verankert. Probleme und Lösungsvorschläge für den Agrarsektor werden z. B. diskutiert in: Robert-Bosch-Stiftung (2000).
} 
Landschaftspflegeverband mit paritätischer Besetzung der Akteure zu errichten. Eine neue Organisation hat zudem den Vorteil, nicht nur ein Anhängsel einer bestehenden Verwaltung zu sein.

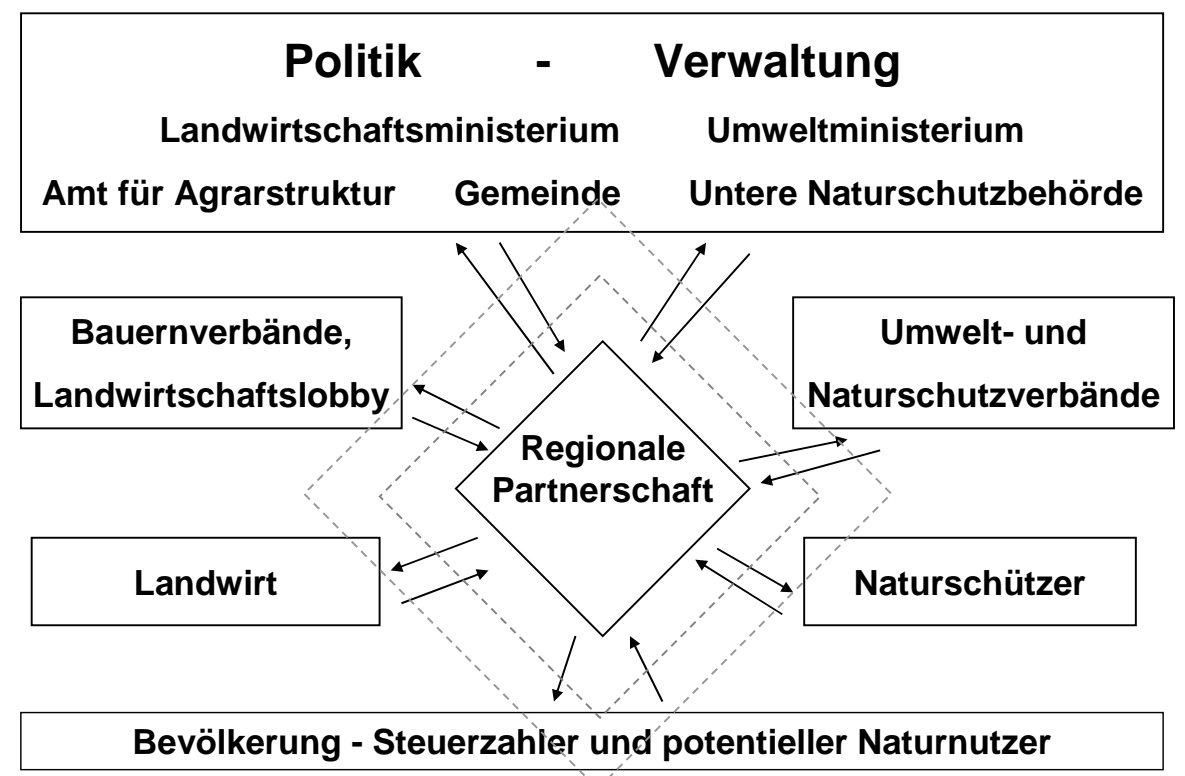

Abbildung 2.4: Wandel der Kommunikationsstrukturen durch eine Regionale Partnerschaft für den Naturschutz in der Kulturlandschaft: Die Regionale Partnerschaft vermittelt zwischen allen Akteuren, moderiert den Dialog und setzt damit Aktivitäten zugunsten der Natur in Gang. Idealerweise werden die Grenzen zwischen den Akteuren und der Regionalen Partnerschaft zusehends undeutlicher, so dass sie nicht als eigenständige Organisation, sondern als Forum zur themenorientierten Zusammenarbeit wahrgenommen wird. Dies ist dann erreicht, wenn die Partnerschaft nicht als weitere konkurrierende Organisation angesehen wird und ihr Aufgaben von verschiedener Seite angetragen werden.

Wenn die Regionale Partnerschaft darauf achtet, mit allen Akteuren gute Kontakte zu pflegen und sie einzubinden, und wenn es ihr gelingt, zum regionalen Promotor für den Naturschutz in der Kulturlandschaft zu werden, dann wird sie zu einem Forum, in dem die Akteure miteinander in Kontakt kommen und sich bei der Bearbeitung von Projekten kennen lernen, um so gegenseitige Vorbehalte abzubauen. ${ }^{21}$

Die Regionale Partnerschaft übernimmt die Projektentwicklung und Mittelakquise. Sie motiviert die Akteure, sich zu engagieren und betreibt die nötige Öffentlichkeitsarbeit. Es ist regional von besonderer Bedeutung, auf den Handlungsbedarf hinzuweisen und aufzuzeigen, welche Möglichkeiten jeder Einzelne und jede Akteursgruppe hat. Es geht darum, die sehr unterschiedlichen bis fehlenden Problemwahrnehmungen zusammenzuführen und so ein gemeinsames Problemverständnis zu entwickeln, um darauf aufbauend $\mathrm{zu}$ gemeinsamen Problemlösungen $\mathrm{zu}$ gelangen. Auf diesem Weg kann das festgestellte Missverhältnis zwischen der wahrgenommenen globalen Umweltkatastrophe und der gleichzeitig wahrgenommenen heilen Heimat überbrückt werden.

\footnotetext{
${ }^{21}$ Für eine ausführliche Diskussion der Erfolgsfaktoren solcher Projekte siehe z. B. Brendle 1999, Heiland 1999.
} 
Darüber hinaus kann diese Partnerschaft die Beteiligung der Bevölkerung organisieren, indem sie repräsentative Befragungen durchführt, offene Beteiligungsforen anbietet und so die Kontaktbarrieren für die Bevölkerung abbaut. Um Bürgern, die nicht in Vereinen, Lobbygruppen oder Verbänden organisiert sind, die Teilnahme an Entscheidungsprozessen zu ermöglichen, ist es nötig, dass sich eine „Beteiligungskultur“ entwickelt. Den Bürgern sollte die Zeit eingeräumt und die nötige Infrastruktur bereitgestellt werden, um Partizipation zu erproben und zu erlernen. Die Bereitstellung dieser Strukturen ist personal- und kostenintensiv. Es muss lokal zumindest eine Personalstelle mit Bürobudget geschaffen werden, zusätzlich müssen die Akteure Zeit investieren und im politischen und Verwaltungsbereich müssten Zuständigkeiten auf die neue regionale Organisation verlagert werden. Würden im jedem der 323 Landkreise in Deutschland 40.000 Euro Budget bereitgestellt, ergäben sich jährliche Kosten von fast 13 Mio. Euro. Agendaprozessen und Naturschutzkreisen mag dies viel erscheinen, für landwirtschaftliche Verhältnisse ist dies aber mit knapp 1\% der jährlich in Deutschland ausgegebenen Fördersumme im Rahmen des EU-Programms zur Förderung der Ländlichen Entwicklung (2. Säule der EU-Agrarpolitik) wenig. Zusätzlich ist zu beachten, dass das Konzept der Regionalen Partnerschaften eine enorme Erhöhung der Effektivität des Umwelt- und Naturschutzes in der Agrarlandschaft ermöglicht, die deutlich über die Chancen von neuen oder verbesserten Maßnahmen hinaus geht.

Weiterhin hat es sich in Niedersachsen bewährt, neue Agrarumweltmaßnahmen zunächst ein Jahr in einer Pilotregion zu testen und die Erfahrungen der Beteiligten (Landwirte, Verwaltungen, Naturschützer) anzuhören. Mit den Erfahrungen einer solchen Pilotphase, die für die Blühstreifen in Niedersachsen im Jahr 2003 im Landkreis Wolfenbüttel im Rahmen des von der Deutsche Bundesstiftung Umwelt (DBU) geförderten Projektes „Blühstreifen als Strukturelemente in der Börderegion Wolfenbüttels“ durchgeführt wurde, gelang dem niedersächsischen Landwirtschaftsministerium im Folgejahr die niedersachsenweite Auflage einer sehr erfolgreichen Förderrichtlinie, die zum einen von den Landwirten stark nachgefragt und auch von allen anderen Akteuren befürwortet und mitgetragen wurde. Die Organisation einer solchen Pilotphase war nur aufgrund der infolge des DBU-Projekts initiierten Regionalen Partnerschaft zu leisten.

\subsection{Fazit}

Wir hoffen, am Beispiel des Agrarbereichs plausibel gemacht zu haben, dass eine dauerhafte und somit nachhaltige Stärkung des Umwelt- und Naturschutzes nur gelingen kann, wenn er von Naturschützern, der Landwirtschaft, den Verwaltungen und den Bürgern gleichermaßen getragen wird. Da es zur Verwirklichung der Querschnittsaufgabe Umwelt- und Naturschutz 
langfristiger Verhaltens- und Einstellungsänderungen bedarf, ist der Weg dorthin mühsam und langwierig. Das vorgestellte Instrument 'Regionale Partnerschaft' kann ein Baustein auf diesem Weg sein, da sie einen Dialog startet und zunächst zwischen allen Akteuren vermittelt, um damit Aktivitäten zugunsten der Natur in Gang zu setzen. Ein kooperatives Vorgehen ist insbesondere deshalb notwendig, um nicht erneut Konflikte um Macht und Finanzmittel auszulösen. Allerdings ist $\mathrm{zu}$ beachten, dass die Regionale Partnerschaft nicht erzwungen werden kann und nur funktioniert, wenn sie gemeinschaftlich getragen wird und einen Mehrwert für die Beteiligten generiert. Ein Anreiz dafür ist jedoch, dass sich EU und Bund entschlossen haben, Regionale Partnerschaften im Rahmen der regionalen Entwicklung zu fördern. ${ }^{22}$

\subsection{Literatur}

Alvensleben, R. v. (2003): Landwirtschaft, Medien und Populismus - Konsequenzen für Kommunikation und Marketing. Archiv der DLG, Band 97, 2003, S. 29-38

Bateman, I. J., R.T. Carson, B. Day, M. Hanemann, N. Hanley, T. Hett, M. Jones-Lee, G. Loomes, S. Mourato, E. Ozdemiroglu, D.W. Pearce, R. Sugden and J. Swanson (2002): Economic valuation with stated preference techniques - A manual, Cheltenham, UK, Northampton, MA, USA.

Beirat BML (1998): Integration der Landwirtschaft der Europäischen Union in die Weltagrarwirtschaft und Kurzstellungnahme, Bonn

Beirat BML (1998a): Agenda 2000: Zunkunftsweg der Agrarpolitik?, Heft 476, Bonn

Beirat BMU (1995): Zur Akzeptanz und Durchsetzbarkeit des Naturschutzes. Natur und Landschaft Jg. 70, Heft 2, S.51-61.

Bertke, E. (2005): Ökologische Güter in einem ergebnisorientierten Honorierungssystem für ökologische Leistungen der Landwirtschaft, Stuttgart

Bertke, E., S. Hespelt, R. Marggraf (2005): Ein regionaler Beirat als partizipatives Gremium in der Agrarumweltpolitik. In Feindt, Newig: Partizipation, Öffentlichkeitsbeteiligung, Nachhaltigkeit. Perspektiven der politischen Ökonomie. Metropolis. S.281-298.

BMU (1993): Bundesministerium für Umwelt, Naturschutz und Reaktorsicherheit: Konferenz der Vereinten Nationen für Umwelt und Entwicklung im Juni 1992 in Rio de Janeiro - Dokumente: Agenda 21, Bonn

BMU (2002): Umweltbewusstsein in Deutschland, Bonn

Brendle, U. (1999): Musterlösungen im Naturschutz - Politische Bausteine für erfolgreiches Handeln , Münster

Ellis R.J., Thompson, M. (Eds.) (1997): Culture Matters, Boulder, Colorado

European Commission, GD Agri (1999): Contribution of the European Community on the Multifunctional Character of Agriculture. EU-Info-Paper, Brussels.

http://europa.eu.int/comm/agriculture/external/wto/document/ip2_en.pdf Zitierdatum: 21.7.05

Europäische Kommission, GD Landwirtschaft (2003): Landwirtschaft und Umwelt. EU-Fact Sheet. Brussels. http://europa.eu.int/comm/agriculture/publi/fact/envir/2003_de.pdf Zitierdatum 10.8.05

Feindt, P.H. (1997): Kommunale Demokratie in der Umweltpolitik - neue Beteiligungsmodelle, in: Aus Politik und Zeitgeschehen. Beilage zur Wochenzeitung Das Parlament, B 27/97, S. 39-46

\footnotetext{
${ }^{22}$ Durch die VO (EG) 1783/2003 zur Änderung der VO (EG) 1257/1999 Ländliche Entwicklung ist in Artikel 33 die Möglichkeit geschaffen worden, Regionale Partnerschaften zu fördern. Primär sind diese neuen Möglichkeiten darauf ausgelegt, die LEADER-Initiativen in die Förderung des ländlichen Raumes zu überführen. Neben Leader, Region aktiv und Agenda Prozessen, besteht aber möglicherweise auch für andere Regionale Partnerschaften, integrierend arbeitende Projekte und Organisationen (Landschaftspflegeverbände) die Möglichkeit zur Förderung.
} 
Freese J., Steinmann H.-H. (2005): Ergebnisse des Projektes „Randstreifen als Strukturelemente in der intensiv genutzten Agrarlandschaft Wolfenbüttels“. Diskussionsbeitrag 0503 des Instituts für Agrarökonomie der Universität Göttingen

http://www.gwdg.de/ uaao/margg/marggraf/team/janfreese/discussionpaper0503.pdf Zitierdatum: 19.9.2006

Gawel, E. (1995): Bürokratietheorie und Umweltverwaltung. Zeitschrift für angewandte Umweltforschung, Jg.8, Heft 1, S.79-89

Heiland, S. (1999): Voraussetzungen erfolgreichen Naturschutzes: individuelle und gesellschaftliche Bedingungen umweltgerechten Verhaltens, ihre Bedeutung für den Naturschutz und die Durchsetzbarkeit seiner Ziele. http://www.ioer.de/PublPDF/heiland_diss_voraussetzungenNat.pdf Zitierdatum: 10.7.2005

Heinze, R.G. (1992). Verbandspolitik zwischen Partikularinteressen und Gemeinwohl, Gütersloh

Henning, C. H. C. A., Glauben, T., Wald, A. (2001): Die Europäische Agrarpolitik im Spannungsfeld von Osterweiterung und WTO-Verhandlungen. Agrarwirtschaft Jg.50 Heft 3, S. 147-152

Henrichsmeyer, W., Grisko, H., Zöller, H (1984): Ökonomische und Ökologische Rahmenbedingungen einer umweltverträglichen Agrarproduktion, in: Informationen zur Raumentwicklung Jg. 1984 Heft 6, S. 513523

Henrichsmeyer, W., Witzke, H.P. (1994): Agrarpolitik, Band 2, Bewertung und Willensbildung, Stuttgart

Hobbensiefken, G. (1991); Ökologieorientierte Volkswirtschaftslehre, 2. Auflage, München u. a.

Jurt, L. (1998) „Ich darf nicht mehr schön finden, was mir gefällt“. Ästhetische Vorstellungen von Bauern und deren Bedeutung für die Biodiversität, in: Agrarsoziologie und Agrarwirtschaft, Nr. 2, S. 125-137 Zürich.

Kluge, U. (1989): Vierzig Jahre Agrarpolitik in der BRD. Hamburg/Berlin

Luz, F. (1994): Zur Akzeptanz landschaftsplanerischer Projekte. Determinanten lokaler Akzeptanz und Umsetzbarkeit von landschaftsplanerischen Projekten zur Extensivierung, Biotopvernetzung und anderen Maßnahmen des Natur- und Umweltschutzes, Frankfurt

Möller 1996: Warum ist Planung so wirkungslos? Naturschutz und Landschaftspflege 28 (6) S.188 f

Olson, M. (1992): Die Logik des kollektiven Handelns. Kollektivgüter und die Theorie der Gruppen,. 3. Aufl., Tübingen.

Oppermann, B. (2000): Konfliktlösungs- und Partizipationsmodelle für eine bürgernahe Naturschutzpolitik, in: BfN (Hrsg): Angewandte Landschaftsökologie, Heft 34, S.37-59

Ott, K. (2002): Akzeptanzdefizite im Naturschutz, in: DRL (Hrsg.): Die veschleppte Nachhaltigkeit: frühe Forderungen - aktuelle Akzeptanz, Heft 74. S.75-81

Pehle, H. (1998): Das Bundesministerium für Umwelt, Naturschutz und Reaktorsicherheit: ausgegrenzt statt integriert? Das institutionelle Fundament der deutschen Umweltpolitik. Wiesbaden

Pongratz, H. (1992): Die Bauern und der ökologische Diskurs. Befunde und Thesen zum Umweltbewusstsein in der bundesdeutschen Landwirtschaft, München, Wien

Reusswig, F. (2003): Naturorientierung und Lebensstile. LÖBF-Mitteilungen Nr. 1/2003, S.27-34

Robert Bosch Stiftung (2000): Agrarumweltpolitik nach dem Subsidiaritätsprinzip. Denkschrift des Schwäbisch Haller Agrarkolloquiums der Robert Bosch Stiftung, Schriften zur Agrarforschung und Agrarpolitik, Bd. 1 , Berlin

Rudloff, B., Urfei G. (2000): Agrarumweltpolitik nach dem Subsidiaritätsprinzip - Kategorisierung von Umwelteffekten und Evaluierung geltender Politikmaßnahmen. Schriftenreihe zur Agrarforschung und Agrarpolitik, Bd. 3, Berlin

Scharpf, F. W. (1991): Die Handlungsfähigkeit des Staates am Ende des zwanzigsten Jahrhunderts, in: Politische Vierteljahresschrift 32 (4), S. 621-634

Schur, G. (1990): Umweltverhalten von Landwirten, Frankfurt a.M.

SRU - Rat von Sachverständigen für Umweltfragen (1985): Umweltprobleme der Landwirtschaft. Sondergutachten 1985, Stuttgart

SRU (2000): Umweltgutachten 2000. Schritte ins nächste Jahrtausend, Stuttgart

SRU (2002a): Sondergutachten 2002. Für eine Stärkung und Neuorientierung des Naturschutzes, Stuttgart 
SRU (2002b): Umweltgutachten 2002. Für eine neue Vorreiterrolle, Stuttgart

SRU (2004): Umweltgutachten 2004. Umweltpolitische Handlungsfähigkeit sichern. Stuttgart

Stoll, S (1999): Akzeptanzprobleme bei der Ausweisung von Großschutzgebieten. Frankfurt (Main) u. a.

Streit M. E., Wildenmann R., Jesinghaus J. (Hrsg.) (1989): Landwirtschaft und Umwelt: Wege aus der Krise, Baden-Baden

Willke (1999): Systemtheorie II: Interventionstheorie. 3. Aufl., Stuttgart 


\section{Kapitel 3}

\section{Zahlungsbereitschaftsanalysen in umweltrelevanten Bewertungsverfahren ${ }^{45}$ \\ Die Eingriffsregelung als Beispiel}

\subsection{Einleitung}

In diesem Kaptitel wird die Anwendbarkeit von Zahlungsbereitschaftsanalysen im Rahmen von Instrumenten der Bewertung und Interpretation von umweltrelevanten Planungen und Vorhaben vorgestellt. Die Analyse und Bewertung der Folgen von Planungen und Vorhaben auf die Bereiche Umwelt, Natur und Landschaft werden im Rahmen verschiedener Verfahren durchgeführt. Gemeinsam ist allen Verfahren, dass eine Bewertung von vorgefundenen Sachverhalten durchgeführt wird. Das heißt, die im Planungsgebiet vorgefundenen und durch die Planung vorgegebenen Sachverhalte sind entscheidungs- und handlungsbezogen $\mathrm{zu}$ bewerten und $\mathrm{zu}$ interpretieren. Zahlungsbereitschaftsanalysen können, wie im folgenden Abschnitt aufgezeigt wird, bei diesem normativen Verfahrenschritt sinnvolle Informationen bereitstellen.

Im Rahmen der gängigen Bewertungsverfahren werden Umwelt, Natur und Landschaft zur Analyse in sog. Schutzgüter zerlegt. Die Bewertung der Schutzgüter Boden, Luft, Wasser, Klima, Tiere, Pflanzen und Biotope findet mittels Verfahren statt, die auf standardisierten naturwissenschaftlichen Methoden basieren. Die naturwissenschaftliche Methodenentwicklung hat eine enorme Vielfalt an Verfahren für diesen komplexen Problembereich hervorgebracht. Nur in einigen Bereichen hat sich ein Verfahren durchgesetzt, häufiger werden allerdings verschiedene Verfahren angewendet und akzeptiert (Lambrecht 1997).

Zunehmend findet jedoch eine Ausdehnung der Bewertungsverfahren auf Bereiche statt, bei denen die Umweltveränderungen auch Auswirkungen auf den Menschen haben. Hierzu

\footnotetext{
${ }^{45}$ veröffentlicht als Buchkapitel Freese, Bräuer (2005): Zahlungsbereitschaftsanalysen in umweltrelevanten Bewertungsverfahren. In: Marggraf, Bräuer, Fischer, Menzel, Stratmann, Suhr: Ökonomische Bewertung bei umweltrelevanten Entscheidungen, Metropolis. S.307-332.
} 
gehören Fragen der Veränderung des Landschaftsbildes, Lärmbelastung durch neue Vorhaben oder die Beeinträchtigung der Erholungsfunktion der Natur für den Menschen.

Im Gegensatz zu den klassisch naturwissenschaftlichen Daten wie Art und Anzahl der betroffenen Biotope, Artenlisten, Flächenverbrauch etc., die direkt im Gelände gemessen werden können, ist dies bei den o. g. menschenbezogenen Sachverhalten nicht mehr möglich. Zwar können Lärmintensität, Quadratkilometer verlorene Erholungslandschaft oder Größe und Sichtbarkeit von Bauwerken erfasst werden, entscheidend ist bei diesen Sachverhalten aber, dass Meinungen, Empfindungen, Einschätzungen und Bewertungen von Menschen über diese Sachverhalte erhoben und in die Bewertungsverfahren eingebracht werden müssen. Dies ist nicht mehr mit den gewohnten Verfahren, die sich im Bereich der Erfassung und Bewertung von den oben genannten naturwissenschaftlichen Daten bewährt haben, zufrieden stellend zu leisten. Sollen die menschenbezogenen Auswirkungen verstärkt im Planungsprozess Berücksichtigung finden, ist nach neuen Bewertungsverfahren zu suchen. Die Methoden

der Zahlungsbereitschaftsanalyse wie die Kontingente Bewertungsmethode (KBM) als etabliertes Verfahren zur Präferenzenmessung der Bürger, bietet sich hier an. Nicht nur, weil die Methode sich sehr flexibel hinsichtlich des zu bewertenden Sachverhaltes erweist, sondern auch, da sie mittlerweile vor amerikanischen Gerichten zur Schadensbewertung zugelassen ist.

Um die Einsatzmöglichkeiten von Zahlungsbereitschaftsstudien $\mathrm{zu}$ verdeutlichen wird im Folgenden am Beispiel der sog. Eingriffsregelung des Bundesnaturschutzgesetztes aufgezeigt, wie Zahlungsbereitschaftsanalysen einen Beitrag zur Bewältigung des Auftrags der umfassenden Analyse und Bewertung von Umweltfolgen leisten können. Dazu werden zunächst kurz wichtige Instrumente umweltbezogener Bewertungsverfahren im Allgemeinen (Abschnitt 3.2) und die Eingriffsregelung im Besonderen (Abschnitt 3.3) kurz vorgestellt. In Abschnitt 3.4 werden dann Möglichkeiten aufgezeigt, die Zahlungsbereitschaftsanalysen bieten, um bisher zu wenig berücksichtigte planungsrelevante Sachverhalte besser in das Planungsverfahren der Eingriffsregelung $\mathrm{zu}$ integrieren. In Exkurs 3.4 .2 werden diese Erkenntnisse exemplarisch auf fachinterne Planungen des Naturschutzes, der Denkmalpflege und des Tourismus übertragen.

\subsection{Die Instrumente umweltbezogener Bewertungsverfahren}

Generell kann zwischen drei Instrumenten der zur Abschätzung der Folgen eines Eingriffes in Natur und Landschaft unterschieden werden: Eingriffsregelung, Umweltverträglichkeits- 
prüfung und Verträglichkeitsprüfung gemäß der EU-Flora-Fauna-Habitat-Richtlinie (FFHVerträglichkeitsprüfung).

Die so genannte Eingriffsregelung ( $\S 18-21$ Bundesnaturschutzgesetz) sichert die flächendeckende Berücksichtigung der Belange des Naturschutzes und der Landschaftspflege bei Vorhaben, Plänen und Projekten. Die Eingriffsregelung wird als Huckepack-Verfahren auf existierende Verfahren wie Aufstellung oder Änderung von Flächennutzungsplänen, Bauplänen, Erteilung von Baugenehmigungen oder im Rahmen von Planfeststellungen aufgesattelt (Gassner 1995).

Zusätzlich wurden zur Prüfung von Plänen und Projekten auf Verträglichkeit mit den Zielen des Europäischen Natura 2000 Schutzgebietsnetzes die FFH-Verträglichkeitsprüfung ( $\S$ 34, 35 Bundesnaturschutzgesetz) und die Umweltverträglichkeitsprüfung (UVP) im Vorfeld eines Zulassungsverfahrens eingeführt. Die UVP ist eine breit angelegte Prüfung der Auswirkungen auf die Umwelt nach dem Umweltverträglichkeitsprüfungsgesetz.

Wie in Abbildung 3.1 dargestellt, berücksichtigen die verschiedenen Instrumente unterschiedliche Schutzgüter und erreichen verschiedene Prüftiefen. Am weitest reichenden sind die Prüfanforderungen in der UVP. Hier werden neben den abiotischen Schutzgütern Boden, Klima, Luft, Wasser, die biotischen Schutzgüter, Biotope, Tier- und Pflanzenarten sowie Auswirkungen auf den Menschen, auf Kultur- und Sachgüter betrachtet.

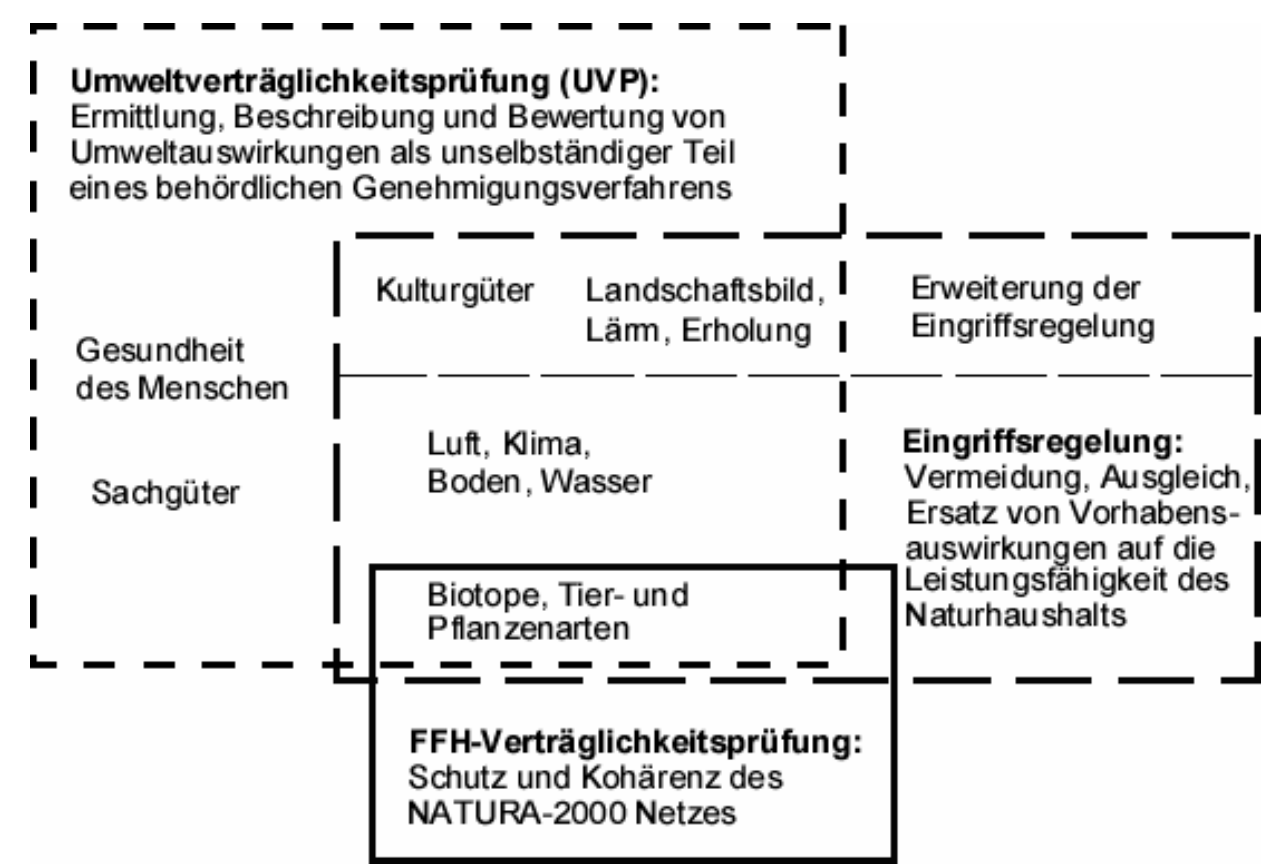

Abbildung 3.1.: Anwendung verschiedener Umweltbewertungsverfahren auf die verschiedenen Schutzgüter. Quelle: nach Breuer 2000, verändert.

Der folgende Abschnitt konzentriert sich auf die Einsatzmöglichkeiten von Zahlungsbereitschaftsanalysen im Rahmen der Eingriffsregelung. Zum einen sind die Einsatzmöglichkeiten 
von Zahlungsbereitschaftsanalysen im Rahmen der UVP bereits an anderer Stelle ausführlich erläutert werden. Zum anderen kommt die FFH-Richtlinie für eine Betrachtung nicht in Frage, da sie sehr scharf ausschließlich auf den Schutz von sog. prioritären Tier- und Pflanzenarten sowie Biotopen von gemeinschaftlich europäischem Interesse abzielt und die Bewertung der Auswirkungen nur für diese Arten und Biotope und nicht für den Menschen und seine Interessen an der Natur vorgenommen wird.

\subsection{Die Eingriffsregelung als Beispiel}

Bevor auf Einsatzmöglichkeiten von Zahlungsbereitschaftsanalysen im Rahmen von Bewertungsverfahren eingegangen wird, soll nachfolgend am Beispiel der Eingriffsregelung das Prinzip derartiger Verfahren aufgezeigt und eine kurze Schematisierung der Umweltbewertungsverfahren vorgestellt werden.

\subsubsection{Prinzip der Eingriffsregelung: Vermeidung, Ausgleich, Ersatz}

Für jeden Eingriffsverursacher gilt das Gebot, die Eingriffsfolgen soweit wie möglich zu vermeiden oder in ihrer Wirkintensität auf die Natur zu minimieren. Für unvermeidbare Eingriffe ist eine angemessene Kompensation ${ }^{46} \mathrm{zu}$ leisten. Die Bewertung der Eingriffsfolgen und der Vorschlag von Vermeidungs-, Ausgleichs- und Ersatzmaßnahmen erfolgt für jeden Eingriff in einem entsprechenden landschaftspflegerischen Fachbeitrag. Diesen hat der Eingriffsverursacher zu erstellen oder erstellen zu lassen. Die Genehmigungsbehörde genehmigt den Fachbeitrag, setzt die Vermeidungs-, Ausgleichs- und Ersatzmaßnahmen z. B. in der Baugenehmigung fest und überwacht die Ausführung. Dies geschieht je nach landesrechtlicher Ausgestaltung im Benehmen oder im Einvernehmen mit der unteren Naturschutzbehörde.

Infokasten 3.1.: Wortlaut der Eingriffsregelung als Auszug aus dem Bundesnaturschutzgesetz

\section{$\S 18$ Bundesnaturschutzgesetz}

1. Eingriffe in Natur und Landschaft im Sinne dieses Gesetzes sind Veränderungen der Gestalt oder Nutzung von Grundflächen oder Veränderungen des mit der belebten Bodenschicht in Verbindung stehenden Grundwasserspiegels, die die Leistungs- und Funktionsfähigkeit des Naturhaushalts oder das Landschaftsbild erheblich beeinträchtigen können.

\section{$\S 19$ Bundesnaturschutzgesetz}

1. Der Verursacher eines Eingriffs ist zu verpflichten, vermeidbare Beeinträchtigungen von Natur und Landschaft zu unterlassen.

\footnotetext{
${ }^{46}$ Da die Unterscheidung zwischen Ausgleich und Ersatz zwar juristisch und naturschutzfachlich gut begründet, aber in der Praxis oft nicht einwandfrei nachvollziehbar ist, bezeichnet Kompensation sowohl Ausgleichs- als auch Ersatzmaßnahmen. Siehe z. B. Hoppe et al. (2000) §18 Rn 62.
} 
Der Verursacher ist zu verpflichten, unvermeidbare Beeinträchtigungen durch Maßnahmen des Naturschutzes und der Landschaftspflege vorrangig auszugleichen (Ausgleichsmaßnahmen) oder in sonstiger Weise zu kompensieren (Ersatzmaßnahmen). Ausgeglichen ist eine Beeinträchtigung, wenn und sobald die beeinträchtigten Funktionen des Naturhaushalts wieder hergestellt sind und das Landschaftsbild landschaftsgerecht wiederhergestellt oder neu gestaltet ist. In sonstiger Weise kompensiert ist eine Beeinträchtigung, wenn und sobald die beeinträchtigten Funktionen des Naturhaushalts in gleichwertiger Weise ersetzt sind oder das Landschaftsbild landschaftsgerecht neu gestaltet ist (...)

\subsubsection{Bewertungsmethoden}

Die im Rahmen der Eingriffsregelung notwendige Bewertung der Leistungs-, Funktions- und Nutzungsfähigkeit des Naturhaushalts und der Tier- und Pflanzenwelt sowie der Vielfalt, Eigenart und Schönheit der Natur erfordert die durch das Vorhaben hervorgerufenen Veränderungen aufzunehmen und entscheidungs- und handlungsbezogen $\mathrm{zu}$ interpretieren und $\mathrm{zu}$ bewerten.

Die Bewertung von Natur und Landschaft ist ein komplexer Vorgang. Daher zerlegen Bewertungsverfahren das Problem analytisch in Teilschritte (siehe Infokasten 3.3) und wenden diese auf Teilbereiche, die Schutzgüter Boden, Klima, Wasser, Flora, Fauna, Biotope, Landschaftsbild, Erholungsfunktion, etc. an. Der Naturbestand muss zunächst aufgenommen, eine Prognose der zu erwartenden Folgen des Eingriffes aufgestellt und dann eine Bewertung der Eingriffsfolgen durchgeführt werden. Das Bewertungsverfahren muss dabei konsistent, nach rationalen Kriterien und nachvollziehbar aufgebaut und angewendet werden.

Im Bereich der Analyse müssen zwei sehr unterschiedliche Arten von Daten verarbeitet werden (siehe Infokasten 3.2). Bei Flächenverbrauch, Biotoptypen, Waldinanspruchnahme, Anzahl der zu fällenden Bäume mit Art, Durchmesser und Zustand handelt es sich um naturwissenschaftliche Daten die aufgenommen und dargestellt werden. Diesen Sachverhalten sind zusätzlich Prozesse, Nutzungen, Funktionen sowie Potential, Belastbarkeit und Tragfähigkeit der Naturgüter zuzuordnen, die nicht direkt aus den Daten abgelesen werden können, sondern aus Forschungsergebnissen abzuleiten sind. Bereits im Rahmen der Analyse dieser Sachverhalte kommt es so zu Wertungen, wenn der Gutachter z. B. festlegen muss, welche Prozesse und Funktionen bedeutend und damit betrachtenswert und welche unbedeutend sind. Die Abschätzung der Folgen der Belastung des Naturhaushaltes erfolgt mit Hilfe der Orientierung an Grenzwerten (minimum-viable-standard-Methode). Dabei wird davon ausgegangen, dass die Verschlechterung von Umweltbedingungen nicht beliebig lange zu einer gleichartigen Verringerung der Leistungs-, Funktions- und Nutzungsfähigkeit des Naturhaushalts führt, sondern dass im Naturhaushalt bei Unterschreiten eines bestimmten Schwellenwertes irreversible Schäden auftreten und infolgedessen Funktionen des Naturhaushaltes zusammenbre- 
chen. Eine direkte Feststellung dieser Grenzwerte ist in aller Regel aber nicht möglich. Stattdessen werden sie von Experten auf Basis von naturwissenschaftlichen, technischen und politischen Vorgaben abgeschätzt.

Nach dieser Einordnung der Sachverhalte ist die Transformation in die Wertebene nötig. Erst die Einstufung als wertvolles oder geringwertiges Biotop, als durch das Projekt akut gefährdete Art oder als nicht ausgleichbare Beeinträchtigung des Landschaftsbildes wird die wissenschaftliche Aussage des Gutachters im Planungsprozess verwertbar. Festzustellen bleibt, dass derartige Wertskalen in einem Prozess lediglich durch Expertengremien, seltener durch Beteiligung von Politik und weiteren gesellschaftlichen Gruppen festgelegt werden (Köppel et al. 1998: 19f).

Zusätzlich zu diesen naturwissenschaftlichen Sachverhalten, sind die menschbezogenen Schutzgüter wie Schönheit des Landschaftsbildes, die Bedeutung von Sach- und Kulturwerten, Auswirkungen von Lärm und Verlust von Naherholungsgebieten zu bewerten. Letztere werden im Folgenden zur besseren Unterscheidung als gesellschaftswissenschaftliche Daten bezeichnet (siehe Infokasten 3.2). Die Unterscheidung ist sinnvoll, da die Daten jeweils verschiedener Erhebungsmethoden bedürfen.

\section{Infokasten 3..2: Definition naturwissenschaftliche und gesellschaftswissenschaftliche Daten}

Zur Vereinfachung werden in diesem Beitrag die Daten und Bewertungen der abiotischen und biotischen Schutzgüter (z. B. Boden, Klima, Wasserhaushalt, Arten, Biotope, Artenlisten und Gefährdungskategorien wie Rote Listen) als naturwissenschaftliche Daten und die der menschbezogenen Schutzgüter (Landschaftsbild, Erholungsfunktion, Identifikationsund Symbolfunktion bestimmter Lebensräume, Tier- und Pflanzenarten) als gesellschaftswissenschaftliche Daten bezeichnet.

Zurzeit wird vielfach versucht, naturwissenschaftliche Verfahren auf den Bereich gesellschaftswissenschaftlicher Daten wie der Frage nach der Schönheit einer Landschaft und ihrem Erholungswert für die Bevölkerung anzuwenden. Dazu wird eine Verschneidung einer technischen Beschreibung des Landschaftsbildes mit der Wahrnehmung eines durchschnittlichen Betrachters durchgeführt. Hier stellt sich die Frage, warum man nicht die Bevölkerung um ihre Einschätzung der Schönheit und die Bedeutung für die eigene Freizeitgestaltung direkt fragt, anstatt den indirekten Weg über durchschnittliche Betrachter und wahrnehmungspsychologische Annahmen zu gehen. Eine direkte Befragung leisten Zahlungsbereitschaftsanalysen, deren Einsatz bei Bewertungsproblemen im Folgenden ausführlicher diskutiert wird. 


\section{Infokasten 3.3.: Schematischer Ablauf der Eingriffsregelung}

Diese unten dargestellte schematisierte Abfolge von Schritten, findet sich in allen Verfahren der Umweltbewertung wieder. Eingegangen wird im weiteren Verlauf dieses Kapitels nur auf die Analyse (Punkt 2) und die Bewertung (Punkt 3).

Bewertungen sind immer in Verfahren eingebettet. Alleine für die Bearbeitung der Eingriffsregelung existiert eine enorme Vielfalt von Bewertungsverfahren ${ }^{47}$. Über die optimale Durchführung der verschiedenen Verfahrensteile und aller Bewertungsschritte im Verlauf der Eingriffsregelung wird immer noch eine umfangreiche Fachdebatte geführt und es gibt viele Änderungs- und Weiterentwicklungsvorschläge (Ott 1997). Folgende Übersicht stellt schematisch die im Rahmen der Eingriffsregelung abzuarbeitenden Teilschritte zusammen.

\section{Eingriffsbestimmung und Festlegung des Untersuchungsraumes und -umfanges sowie der Methodik und der Grundsätze}

Nach Feststellung der Möglichkeit eines Eingriffs durch ein Vorhaben ist der Untersuchungsraum, Umfang und Methodik der Untersuchungen, die Auswahl von Indikatoren und die Darstellung der Ergebnisse festzulegen. Dies bestimmt die Aussagekraft und Detailschärfe der Bestandsaufnahme und anschließenden Bewertung. Festgelegt wird hierdurch, welcher Ausschnitt vom Untersuchungsobjekt betrachtet wird.

\section{Schutzgutbezogene Bestandesanalyse und Erhebung der Eingriffsfolgen}

Aufgrund von Geländeerhebungen, Literatur- und Kartenauswertungen sind die aktuellen Ausprägungen der Schutzgüter zu erheben. Weiterhin besteht die Aufgabe festzustellen, welche Biotope, Tier- und Pflanzenarten betroffen sind. Je nach Schwere des Eingriffs und somit der Betroffenheit besonderer Naturfunktionen sind dafür qualitative und quantitative Daten verschiedener Auflösung nötig. Häufig werden Indikatoren eingesetzt, die Aussagen zu Naturzuständen erlauben, ohne eine Komplettanalyse durchführen zu müssen.

Der Wert von Natur für den einzelnen Menschen, in Form des Erholungswertes einer Landschaft und als Wert des Landschaftsbildes, sind nur schwer wie die vorgenannten Sachverhalte mit Hilfe von Indikatoren ermittelbar. Bisher kommen hier Annahmen über die Auswirkungen zum Zuge (Expertenmeinung). Hier wird im weiteren Verlauf des Kapitels vorgeschlagen, Zahlungsbereitschaftsanalysen zu nutzen.

\section{Bewertung der Eingriffsfolgen}

Die im 2. Schritt erhobenen Sachverhalte stellen noch keine Bewertung der Naturausstattung und Eingriffsfolgen dar. Dies erfolgt in einem 3. Schritt, bei dem die Tatbestände und fachwissenschaftliche Hintergründe verschiedener Fachdisziplinen in Bewertungen wie „selten“, „,bedroht“, „gefährdet“, „empfindlich“ oder „ökologisch wertvoll“ übersetzt werden. Diese Wertungen basieren sowohl auf fachwissenschaftlichen Erkenntnissen, als auch auf Wertmaßstäben, die durch Einschätzung und Setzung von Expertinnen entstanden sind (z. B. Roten Listen).

Zentrale Aufgabe der Bewertung ist weiterhin, mögliche Wechselwirkungen zwischen den Teilbeeinträchtigen zu identifizieren und in ihren Auswirkungen abzuschätzen.

Die Gesamtbewertung eines Vorhabens erfolgt durch Betrachtung der Teilergebnisse der einzelnen Schutzgüter und ihrer Wechselwirkungen. Die Verschiedenartigkeit der Schutzgüter und der verwendeten Indikatoren und Verfahren erlaubt es nicht, die verschiedenen Teilergebnisse zu einem Gesamtergebnis zusammen zu fassen.

\section{Erarbeitung von Vermeidungs- und Kompensationsmaßnahmen}

Auf der Grundlage der Bewertung der Eingriffsfolgen muss der Eingriffsverursacher

\footnotetext{
${ }^{47}$ Übersichten und Vergleiche z. B. bei LANA 1996, Jessel 1996, Hoppenstedt 1996, Schweppe-Kraft 1998, Plachter et al. 2002, Lambrecht 1997.
} 
naturale Kompensationsmaßnahmen erarbeiten, die die Wirkungen des Eingriffs ausgleichen.

\section{Bilanzierung und Bewertungsprüfung}

Die so ermittelten Informationen und Maßnahmen sind vom Eingriffsverursacher in einem Landschaftspflegerischen Begleitplan darzulegen. Darin müssen die Ausgangsbedingungen dargestellt, die zu erwartenden Beeinträchtigungen erläutert, Vorhabensvarianten gegeneinander abgewogen, Vermeidungs-, Ausgleichs- und Ersatzmaßnahmen vorgeschlagen und bilanzierten werden.

Dieser Fachplan dient der Genehmigungsbehörde als Entscheidungsgrundlage. Sie prüft, ob die vorgenommene Bewertung vollständig und korrekt ist, ob alle zumutbaren Vermeidungsmaßnahmen aufgezeigt sind und ob die angebotenen Kompensationsmaßnahmen die Eingriffsfolgen kompensieren können.

\subsubsection{Weiterentwicklung der Eingriffsregelung: Ausgleichs- zahlungen und Monetarisierung der Eingriffsfolgen}

Instrumente wie die Eingriffsregelung werden entsprechend den sich ändernden Erfordernissen angepasst und weiterentwickelt. Unter den aktuell diskutierten Entwicklungslinien finden sich einige Vorschläge, die eine Tendenz zur Monetarisierung der Eingriffsfolgen aufweisen. Diese sollen kurz vorgestellt werden ${ }^{48}$.

Grundsätzlich ist es nicht notwendig, im Lauf der Eingriffsreglung Kosten des Natureingriffs abzuleiten. Es gibt jedoch Fälle, in denen dies sinnvoll ist. Unumgänglich ist eine Monetarisierung, wenn der naturale Ausgleich, also die Durchführung der Kompensationsmaßnahmen, nicht realisierbar ist, da z. B. die nötigen Flächen nicht verfügbar sind ${ }^{49}$. Dann kann dem Vorhabensträger eine Geldleistung abverlangt werden (Ausgleichsgeld), die anderen Naturschutzmaßnahmen oder einem Naturschutzfond zufließt. Diese Möglichkeit wird vielfach von Bauherren präferiert, da ihnen eine Geldzahlung einfacher erscheint, als die Ausführung der Kompensationsmaßnahme. Zusätzlich wird von naturschutzfachlicher Seite immer wieder überlegt, ob es nicht sinnvoll und effektiver ist, anstatt vieler kleiner Kompensationsmaßnahmen einige größere Naturschutzvorhaben in Regie des Naturschutzes durchzuführenden. Für diese Maßnahmenbündelung wäre die Ermittlung von Geldbeträgen sehr hilfreich. Der Infokasten 3.4 stellt die Grundzüge der Hessischen Ausgleichsabgabenverordnung als ein existierender Ansatz zu Monetarisierung von Eingriffsfolgen dar.

\footnotetext{
${ }^{48}$ Für eine ausführliche Diskussion der Vor- und Nachteile einer generellen Ausgleichsabgabe siehe SchweppeKraft (1998), S. 215ff.

${ }^{49} \mathrm{Zu}$ unterscheiden ist hier zwischen der Nicht-Ausgleichbarkeit und somit der Nicht-Genehmigungsfähigkeit des Eingriffs aufgrund seiner Eigenart und Schwere (z. B. bei der Zerstörung eines Moores, das innerhalb der üblichen 25 Jahre nicht wieder hergestellt werden kann) und der rechtlichen Verhinderung des Ausgleichs z. B. aufgrund der Nichtverfügbarkeit der Ausgleichsfläche oder des Verbots, die nötigen Ausgleichsmaßnahmen durchzuführen (so kann der Ausgleich von Waldrodungen durch Aufforstungen dadurch ausgeschlossen sein, dass in waldreichen Gebieten oder besonderen waldfreien und waldfrei zu haltenden Regionen Aufforstungen aus naturschutzfachlichen Gründen untersagt sind).
} 
Das Bayerische Landesamt für Umweltschutz (1998) pflegt die „Kostendatei für Maßnahmen des Naturschutzes und der Landschaftspflege“, aus der die Herstellungskosten für Biotoptypen und Lebensräume abzulesen sind. Seit 1992 wurden in zwei Forschungsvorhaben des Bundes $^{50}$ Grundlagen für eine Ausgleichsabgabenregelung in Form eines Maßnahmen- und Kostengerüsts entworfen. Schweppe-Kraft (1998) hat den Anlauf unternommen, ein Modell zur Monetarisierung von Eingriffsfolgen zu erarbeiten.

Auch wenn Landesnaturschutzgesetze in bestimmten Situationen zugunsten der Zahlung einer zweckgebundenen Ausgleichsabgabe auf die Ausführung der Ausgleichsmaßnahme verzichten (Übersicht zur Ausgleichsabgabe z. B. in Köppel et al. (1998) S. 232ff), hat bisher einzig Hessen 1981 für die Eingriffsregelung obligatorisch zusätzlich zur gutachterlichen Bewertungsebene eine landesweit einheitliche monetäre Bewertung eingeführt (siehe Infokasten $3.3)$.

Der Schwerpunkt aller Verfahren zur Monetarisierung liegt im Bereich des Arten- und Biotopschutzes. Sie konzentrieren sich also auf die naturwissenschaftlichen Daten. Trotz des Aufwandes, den diese Verfahren betreiben, bleibt das Kernproblem der Wertzuweisungen erhalten. Welchen Wert hat ein Baum, ein Kilometer Hecke, ein Greifvogelrevier in dem regelmäßig Jungvögel erfolgreich aufgezogen werden oder eine Feuchtwiese mit Trollblumenbestand? Grundlage der Preisbildung sind bisher die sog. Wiederherstellungskosten, also der Preis, der für die Durchführung der nötigen Kompensationsmaßnahmen zu zahlen wäre. Dieser wird dann, je nach Verfahren z. B. aufgrund des Alters des betroffenen Biotops, modifiziert. Preisfestsetzungen für Schäden am Landschaftsbild oder der Erholungsfunktion werden ebenfalls gutachterlich mittels einer aus naturwissenschaftlichen Verfahren abgeleiteten Methode erhoben (Hessische Zusatzbewertung Landschaftsbild 1995). Dieser angebotsorientierte Ansatz liefert sicherlich einen wichtigen ersten Ansatz. Der gesellschaftliche Wert ist aber allein über den Nutzen, den ein Umweltgut stiftet bestimmt und nicht über die Kosten seiner Bereitstellung (Marggraf \& Streb 1997).

Gerade im Bereich der Wertermittlung der auf Menschen ausgerichteten Naturfunktionen werden die Schwächen diese den Naturwissenschaften entliehenen Verfahren offenbar. Die Schönheit und Unversehrtheit des Landschaftsbildes bewertet jeder Mensch individuell, ebenso, wie jeder die Erholungsfunktion der Eingriffsregion aufgrund seiner eigenen Freizeitgewohnheiten und regionalen Vorlieben unterschiedlich schätzt. Hier mit durchschnittlichen Wahrnehmungen und durchschnittlichem Freizeitverhalten zu arbeiten, wird der Sache nicht gerecht, zumal im Bereich der menschbezogenen Funktionen die besondere Chance besteht,

\footnotetext{
${ }^{50}$ Schemel et al. (1995) und Bosch \& Partner (1993).
} 
die Menschen nach ihrer Einschätzung zu fragen. Genauso wie Annahmen in den Naturwissenschaften als potentielle Fehlerquellen gelten und nur bei fehlenden Primärdaten akzeptiert werden, sollten auch im Bereich gesellschaftswissenschaftlicher Daten Annahmen so weit wie möglich vermieden werden. Es ist gut nachvollziehbar, dass die Wertzuweisungen bei biotischen und abiotischen Schutzgütern nach naturschutzfachlichen Gesichtspunkten durch Gutachter festgesetzt werden, da hier alleine ein Wert aus Sicht einer Fachdisziplin abgebildet werden soll. Nicht plausibel ist aber ein gutachterliches Vorgehen bei der Wertermittlung, wenn gerade Wertschätzungen und Nutzungen vom Menschen erfasst werden sollen. Hier bleibt nur der Weg, die Menschen nach ihrer Wertschätzung zu fragen.

Infokasten 3.4.: Ausgleichsabgabenverordnung (AAV) Hessen - Erste Schritte der monetären Umweltbewertung in der Praxis der Eingriffsregelung

Zur Vereinheitlichung der monetären Bewertung der Eingriffe wurde in Hessen 1991 das so genannte „Biotopwertverfahren“ entwickelt und die Berechnung der Ausgleichsabgabe direkt an diese Bewertungsmethode gekoppelt. 1995 wurde dieses Verfahren als Ausgleichsabgabenverordnung (AAV) verbindlicher Teil der Abarbeitung der Eingriffsregelung in Hessen.

Leitidee des Biotopwertverfahrens ist, diejenigen Eingriffsverursacher, deren Eingriffe nicht ausgeglichen werden können ${ }^{51}$, nicht besser zu stellen als diejenigen, deren Eingriff ausgeglichen werden können und damit auch ausgeglichen werden müssen. Der strikte ökonomische Ansatz der AAV führt dazu, dass alle in Mitleidenschaft gezogenen Werte und Funktionen des Naturhaushaltes als Berechnungsgrundlage herangezogen werden. Die Bewertung der betroffenen Biotope mündete in einer Anzahl von Biotopwertpunkten. Durch Korrekturfaktoren (Landschaftsbild, Zerschneidung von Lebensräumen, Randstöreffekte, Klimawirkungen, besondere örtliche Wirkungen) werden mittelbare Störungen, die nicht biotop- und flächenbezogene sind, erfasst. Die geplante Ausgleichsmaßnahme wird mit derselben Methode bewertet. Die Differenz zwischen Bewertung der Ausgangssituation und der angebotenen Ausgleichsmaßnahme zeigt an, ob der Eingriff vollständig ausgeglichen ist.

Das Besondere an der hessischen Methode ist, dass auf diese Bewertungsebene noch eine monetäre Ebene aufgesattelt wird. Der Geldwert eines Biotopwertpunktes wird durch den sog. Rekultivierungserfolgsindex (REI) festgelegt. Der REI soll regelmäßig aufgrund der Evaluation von durchgeführten Kompensationsmaßnahmen aktualisiert und so die kontinuierliche Anpassung der Ausgleichsabgabe an die Preisentwicklung sichergestellt werden. Somit wird der Differenz zwischen Ausgangszustand und Kompensationsmaßnahme ein Geldwert zugeordnet, der zweckgebunden zur Förderung von Natur und Landschaft durch den Vorhabensträger zu entrichten ist. Werden gar keine Ausgleichsmaßnahmen angeboten, kann hierfür ebenfalls eine Geldsumme festgesetzt werden.

\footnotetext{
${ }^{51}$ siehe Fußnote 49.
} 


\subsection{Einsatzmöglichkeiten von Zahlungsbereitschafts- analysen im Rahmen der Eingriffsregelung}

\subsubsection{Die Ermittlung gesellschaftlicher Werte}

Die Zahlungsbereitschaftsanalyse soll im Folgenden als eine wertvolle Ergänzung für die Bewertung gesellschaftswissenschaftlicher Daten vorgestellt werden. Dies gilt insbesondere für die Messung individueller Präferenzen. Die Bewertung der Beeinträchtigung des Landschaftsbildes oder der Erholungsfunktion durch einen Eingriff stellt, egal ob es sich um einen Sendemast, eine Windkraftanlage, ein Hochhaus, eine Industrieanlage oder ein Stallgebäude handelt, eine Summation individueller und subjektiver Bewertungen dar ${ }^{52}$. Daher ist es nicht plausibel, dass z. B. Fragen der Wiederherstellung des Landschaftsbildes durch Experten bewertet werden. Der Entwurf einer neu gestalteten Landschaft wird heute vielfach schon visuell simuliert ${ }^{53}$. Diese Simulationen sollten von einem repräsentativen Bevölkerungsausschnitt bewertet werden. Landschaftsbild und Erholung in der Natur sind auf die Menschen ausgerichtete Schutzgüter, daher sollte es auch den Menschen überlassen bleiben, zu urteilen, ob die Wiederherstellung eines Landschaftsbildes oder die Neugestaltung einer Erholungslandschaft gelungen ist.

Bisher wird analog zur Indikatorennutzung im Bereich naturwissenschaftlicher Daten der biotischen und abiotischen Schutzgüter versucht, Bewertungen menschbezogener Sachverhalte aufgrund der Maßstäbe eines durchschnittlichen Betrachters durchzuführen. Unter Bezugnahme auf quantifizierbare Kriterien wie z. B. Ausmaße eines Gebäudes, Beschaffenheit der Wandflächen oder Höhe eines Mastes und die Zugrundelegung einiger wahrnehmungspsychologischer Annahmen, wie z. B. dass ein Hochhaus oder Gebäudekomplex in einer urbanen Umgebung sehr viel weniger beeinträchtigend wahrgenommen wird, als ein Vorhaben, das inmitten einer unbebauten Naturlandschaft liegt, wird eine Bewertung hergeleitet, ohne die betroffenen Menschen konkret nach ihrer Bewertung zu fragen.

Immer dort, wo diese Verfahren bisher angewendet werden, bietet sich die Nutzung von Zahlungsbereitschaftsanalysen als Alternative an. Sie benötigen keine a priori Festlegung der

\footnotetext{
52 „Die Wahrnehmung von Natur und Landschaft ist immer subjektiv, da ein wahrnehmendes Subjekt vorhanden sein muss; zudem ist Wahrnehmung immer grundsätzlich selektiv und damit wertend ... Obwohl eine sozialwissenschaftliche oder philosophische Herangehensweise an diese Fragen problemadäquater wäre, zwingen uns gesellschaftliche Rahmenbedingungen und die Systematik der Gesetzgebung dazu, das naturwissenschaftliche Methodenrepertoire anzuwenden.“(Köhler, Preiß 2000: 7).

${ }^{53}$ Zum Beispiel Forschungs- und Entwicklungs-Vorhaben "Erarbeitung von Ausgleichs- und Ersatzmaßnahmen für die Wert- und Funktionselemente des Landschaftsbildes" am Lehrstuhl für Landschaftsökologie, Universität Potsdam.
} 
durchschnittlichen Wahrnehmung. Durch die Befragung einer repräsentativen Zahl von Menschen kann ermittelt werden, welche Wertschätzung dem Bewertungsgut entgegengebracht wird. Das Ergebnis beruht nicht auf der individuellen Einschätzung eines Experten, sondern integriert über ein standardisiertes Verfahren eine repräsentative Vielzahl subjektiver Bewertungen und ermöglicht, neue, im bisherigen Verfahren nicht erfasste Aspekte zu berücksichtigen.

Diese Vorteile der Zahlungsbereitschaftsanalyse können in die verschiedenen Instrumenten der Umweltbewertung eingebracht werden.

\section{Infokasten 3.5.: Wertdimensionen der Natur}

\section{Naturschutzfachlicher Wert, kulturhistorischer Wert (fachdisziplinäre Wertschät- zung)}

Diese Fachbewertungen ergeben sich nach Bewertungsverfahren, die innerhalb des Naturschutzes oder des Denkmalschutzes entwickelt wurden.

\section{2. menschbezogener, gesellschaftlicher Wert}

Um zu verdeutlichen, dass der gesellschaftliche Wert von Naturgütern höher ist, als der reine finanzielle Ertrag, beispielsweise der land- oder forstwirtschaftlichen Nutzung, ist das Konzept des Ökonomischen Gesamtwerts (Total Economic Value, TEV) entwickelt worden. Der TEV (UNEP 1996: 830) wird entsprechend der Motivation der Wertschätzung unterteilt und setzt sich aus nutzungsabhängigen und nutzungsunabhängigen Werten zusammen (siehe Abb. 3.2.). Die nutzungsabhängigen Werte resultieren entweder aus einer direkten Nutzung, sei es die wirtschaftliche Nutzung von Biomasse durch Land-, Forst- und Fischereiwirtschaft oder dem Konsum von Natur in Form von Erholung und Naturbeobachtung oder aus indirekten Werten, die sich aus ökologischen Funktionen wie dem Hochwasserschutz oder dem Selbstreinigungspotential eines Gewässers ergeben. Bei den nutzungsunabhängigen Werten werden Existenz- und Vermächtniswerte unterschieden. Von einem Existenzwert spricht man, wenn allein das Wissen um die Existenz des Umweltgutes Nutzen stiftet. Der Vermächtniswert ergibt sich aus dem religiös, heimatlich oder anders motivierten Bestreben der Individuen, die Umwelt in einem bestimmten Zustand an nachfolgende Generationen weiterzugeben. 


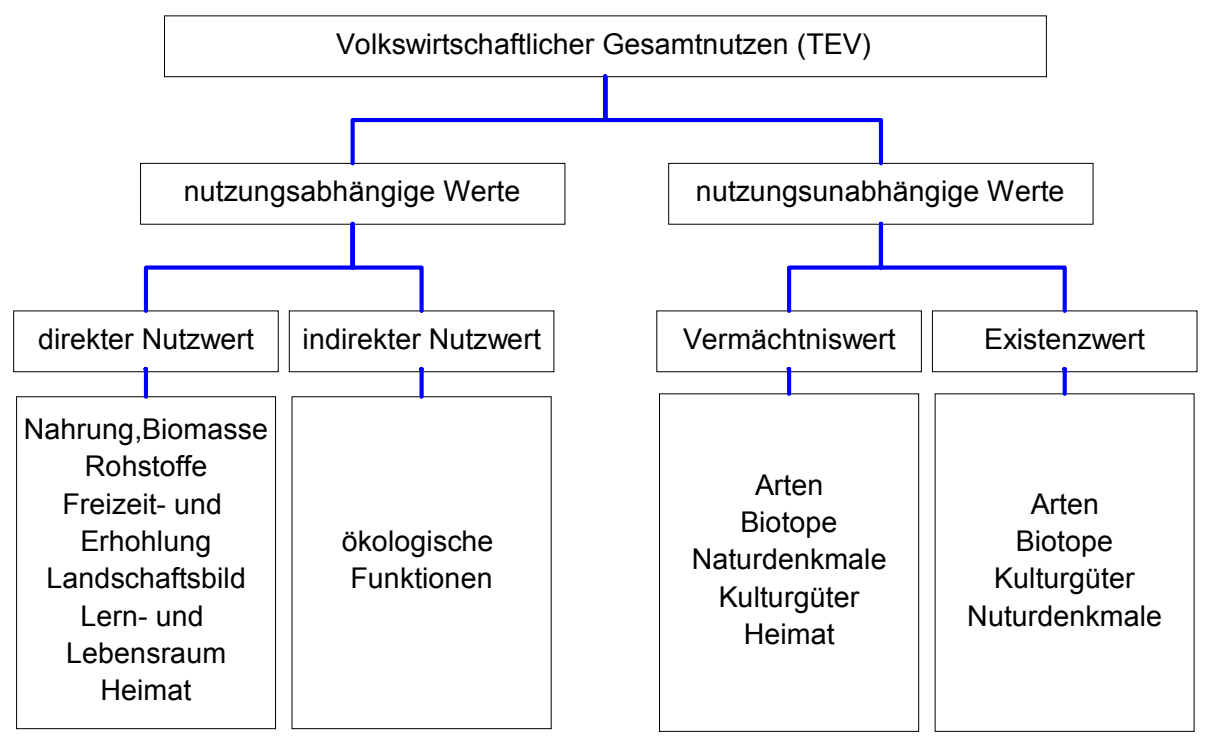

Abbildung 3.2.: Zusammensetzung des volkswirtschaftlichen Gesamtnutzens. Quelle: nach Turner (1991), verändert.

Anschaulich wird die Notwendigkeit, den gesellschaftlichen Wert bei Umweltbewertungsverfahren zu berücksichtigen, wenn man z. B. die Bedeutung eines stadtnahen Waldes ermitteln will. Dieser hat neben dem naturschutzfachlichen Wert, der sich aus dem Vorhandensein von Arten und Biotopen der Roten Listen, der Gebietsgröße und -struktur und dem Beeinträchtigungsgrad ergeben, vielfältigen für den Menschen relevanten Nutzen. Dieser ergibt sich z. B. aus seiner Bedeutung für das lokale Klima, seinem Erholungswert oder wenn zusätzlich die landschaftliche Schönheit des Waldes geschätzt wird. Darüber hinaus kann er einen besonderen Identifikations- und Erfahrungsraum für die Stadtbevölkerung darstellen (z. B. Englischer Garten in München, Tiergarten in Berlin, Alster oder Planten \& Bloomen in Hamburg, Stadtwald Köln) und kann als Symbol der Heimat wertgeschätzt werden.

Entsprechend ist der Kölner Stadtwald für die Kölner erheblich mehr als die Summe seiner Bäume und niemand würde sich damit zufrieden geben, bei Überbauung des Englischen Gartens $10 \mathrm{~km}$ isarabwärts einen neuen Park anzulegen. Der Ausgleich der ökologischen Funktionen dieser Gebiete würde offensichtlich zu einer erheblichen Unterkompensation eines Eingriffs im Bereich menschenbezogener Schutzgüter führen. Dies wurde bisher auch schon erkannt und mittels Einsatzes von Wichtungsfaktoren berücksichtigt. Mit Zahlungsbereitschaftsanalysen jedoch stünde ein Instrument bereit, das eigens zur Wertermittlung solcher Zusatzwerte von der Umweltökonomie entwickelt worden ist.

Findet zusätzlich eine Identifikation und damit eine Wertschätzung des lokalen Waldes statt, ohne dass die betreffende Person diesen tatsächlich nutzt, handelt es sich um eine sog. nutzungsunabhängige Wertschätzung (siehe Abb. 3.2.). Auch diese Wertekategorie lässt sich 
mit Hilfe der Zahlungsbereitschaftsanalyse erfassen.

Es erscheint daher sinnvoll immer dann, wenn im Verfahrensverlauf Hinweise und Anhaltspunkte auftauchen, dass das Planungsgebiet einem erheblichen Bevölkerungsanteil „etwas besonders“ bedeutet, Zahlungsbereitschaftsanalysen zur Ermittlung dieser Wertschätzung einzusetzen, in diesem Fall also die Eingriffsregelung zu erweitern (siehe Abb. 3.3.). Für Gebiete, die einen solchen Mehrwert, z. B. in Form eines Existenzwertes besitzen oder als Identifikations- und Erfahrungsraum der lokalen Bevölkerung besonders geschätzt werden und damit direkt Einfluss auf die Lebensqualität der Menschen haben, sind im Rahmen der Eingriffsregelung verstärkt die menschenbezogenen Schutzgüter zu betrachten.

Erholungsfunktion und Freizeitwert, Unversehrtheit des Landschaftsbildes, Heimat, Lebensund Lernraum sind wichtige Funktionen des Naturhaushalts und damit planungsrelevante Sachverhalte. Da die Beispiele nicht immer so eindeutig wie im Fall des Englischen Gartens oder der Hamburger Alster sind, werden diese Aspekte regelmäßig unterbewertet oder überhaupt nicht beachtet. Wie schätzt die Bevölkerung z. B. das Moor oder den Wald in der Umgebung ihrer mittelgroßen Stadt bezüglich der Erholungsfunktion ein? Soll die Umgehungsstraße westlich an der Stadt vorbeigeführt werden und dabei eine Flussaue beeinträchtigen? Welche Präferenzen haben die Einwohner?

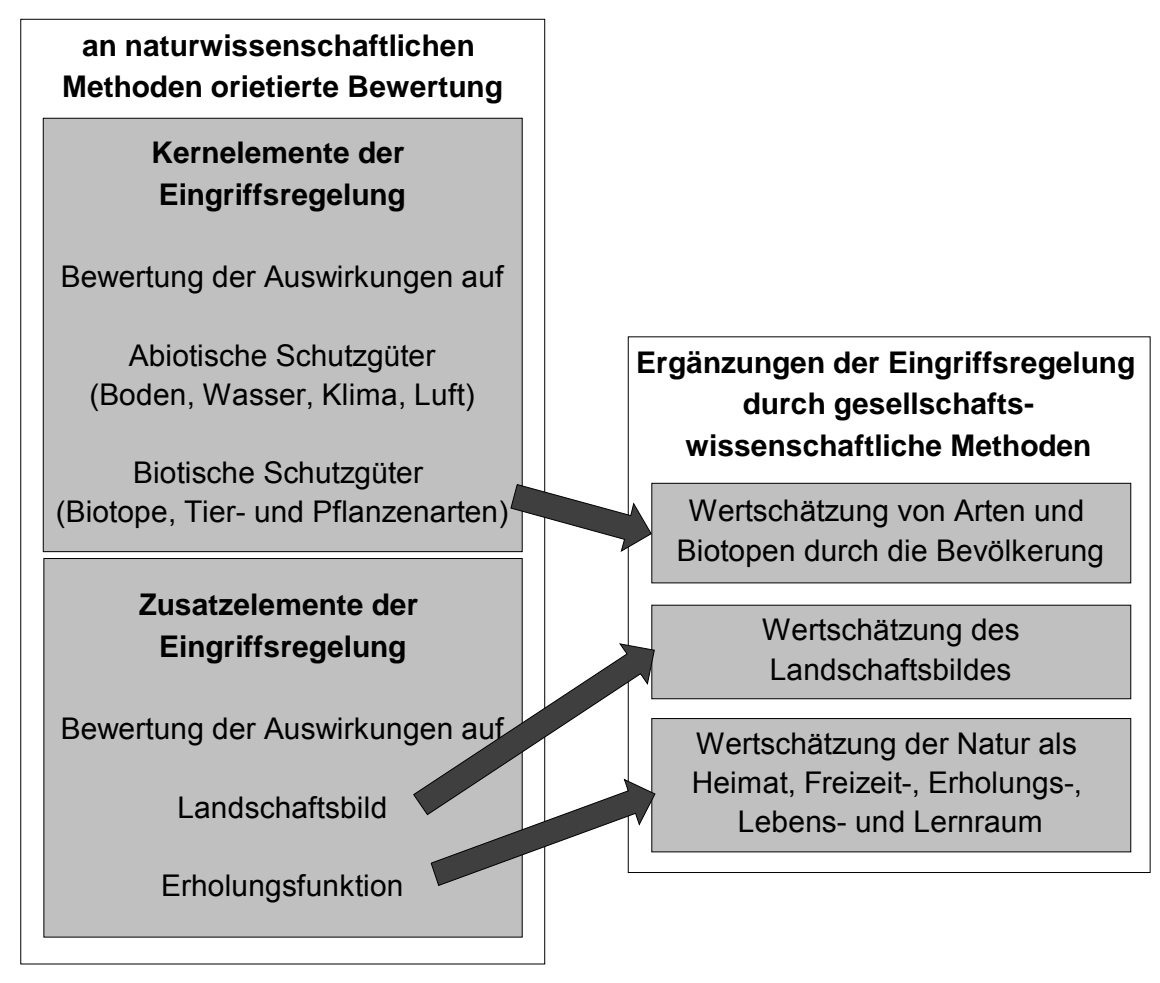

Abbildung 3.3.: Erweiterungen der Eingriffsregelung zur Berücksichtigung der Wertschätzung der Natur. Quelle: eigene Darstellung. 
Ein weiteres Einsatzgebiet von Zahlungsbereitschaftsanalysen kann die Ermittlung der Wertschätzung lokaler Populationen bestimmter Tier- und Pflanzenarten durch die Bevölkerung sein. Neben dem naturschutzfachlichen Wert können bestimmte Arten aufgrund ihres Symbolcharakters einen zusätzlichen Wert für die Bevölkerung besitzen. Hat der Seehund für die Insel Wangerooge eine besondere Bedeutung? Unterliegen die Orchideenwiesen oder die mit Adonisröschen bewachsenen Hänge einer besonderen Wertschätzung durch die ansässige Bevölkerung? Für die Bewohner von Biberach hat möglicher Weise das Vorhandensein des Bibers im Gewässersystem der Region eine besondere Bedeutung und die Einwohner entlang der touristisch vermarkteten „Storchenroute“ bringen den Störchen und ihrem Lebensraum eine höhere Wertschätzung entgegen als der Durchschnittsbürger, bzw. reagieren besonders empfindlich auf eine Beeinträchtigung dieser Arten durch bestimmte Maßnahmen. Diese Wertschätzung kommt aufgrund einer stärkeren Identifikation mit diesen Tieren in der jeweiligen Region zustande. Dem muss bei der Eingriffsbewertung Rechnung getragen werden indem die Gebietskulisse, also der lokale Kontext, eine stärkere Berücksichtigung findet. Dies könnte sogar soweit führen, dass sich unter Einbeziehung dieser Wertschätzungen eine andere Abwägung zwischen den Varianten eines Vorhabens ergibt. Auf alle Fälle aber erhöht eine besondere regionale Wertschätzung den Kompensationsbedarf des zu bewertenden Vorhabens.

Theoretisch können die beschriebenen besonderen Wertschätzungen über jede Art der Bürgerbeteiligung identifiziert werden. Eine besondere Stärke von Zahlungsbereitschaftsanalysen im Vergleich zu klassischen Umfragen ist jedoch, dass sie nicht nur ein qualitatives $\mathrm{Maß}$ liefert, sondern auch eine in Geld ausgedrückte Quantifizierung der Wertschätzung leisten. Dies kann z. B. nötig werden, wenn Varianten eines Vorhabens zu prüfen sind und beide einen ähnlichen naturschutzfachlichen Wert besitzen. Zwei Gebiete sind naturnahe Naherholungsgebiete, doch welches Gebiet wird von der Bevölkerung als höherwertig bewertet? Oder es gibt zwei Gestaltungsvarianten innerhalb eines Gebietes. Für welches entscheidet sich die Bevölkerung? Hierauf kann die Zahlungsbereitschaftsanalyse bei richtiger Anwendung eine Antwort liefern.

Im Bereich der Auswirkungen von „Lärm im Freien“ auf den Menschen ist bei der Forschreibung der Bewertungsverfahren im Rahmen der Bundesverkehrswegeplanung neben dem Vermeidungskostenansatz auch die Zahlungsbereitschaftsanalyse als Verfahren anerkannt worden (Penn-Bressel 2001). Die Bundesverkehrswegeplanung muss Vorhaben nach ihrer Wirtschaftlichkeit bewerten, benötigt also monetäre Aussagen, um die Kosten eines Vorhabens dem volkswirtschaftlichen Nutzen gegenüber zu stellen. Nach vielen Jahren Diskussion 
hat sich hier der Grundsatz durchgesetzt, dass Lärm sehr individuell wahrgenommen wird und die Zahlungsbereitschaftsanalyse ein adäquates Verfahren zur Schadensermittlung darstellt.

Der Sachverständigenrat für Umweltfragen hat in seinem Sondergutachten 2002 fordert, dass Kompensationsmaßnahmen neben der Funktionssicherung beeinträchtigter Naturfunktionen auch der betroffenen Bevölkerung zu Gute kommen sollen (SRU 2002: Tz. 324). Dies erfordert aber zunächst die Ermittlung der Präferenzen der lokalen Bevölkerung.

\subsubsection{Exkurs: Zahlungsbereitschaftsanalysen als Ergänzung zu fachinternen Planungen}

Eine weitere praktische Anwendung von Zahlungsbereitschaftsanalysen kann im Bereich von fachinternen Planungen erfolgen. Solche fachinternen Planungen sind strategische Planungen und entwickeln die Handlungsgrundsätze und -leitlinien einer Fachbehörde (siehe auch Kap. 4). Diese Planungen sind nicht mit den Interessen anderer Behörden oder Abteilungen abgestimmt, sondern stellen den fachlichen Idealzustand ohne die Notwendigkeit, Kompromisse machen zu müssen, dar. Diese Fachplanungen dienen der Fachbehörde als Entscheidungsgrundlage und leisten eine Priorisierung und Fokussierung des Mittel- und Arbeitseinsatzes.

Im Bereich von touristischen Fachplanungen kann die oben vorgestellte Möglichkeit, mit Hilfe von Zahlungsbereitschaftsanalysen den Wert der Erholungsfunktion zu quantifizieren, genutzt werden. Bei denkmalschützerischen Fachplanungen bietet sich die Inwertsetzung eines Kulturgutes aufgrund des Symbolcharakters als Ergänzung zur fachwissenschaftlichen Bewertung an.

Bei Naturschutzfachlichen Planungen (Ökologische und landschaftspflegerische Gutachten, Schutzgebietsgutachten, Landschaftsrahmenplanungen oder Landschaftspläne) spielen bisher überwiegend ökologische Gesichtspunkte, die im Kriterium Schutzbedürftigkeit (Sensibilität und Störungsempfindlichkeit) münden, eine wichtige Rolle. Es zeigt sich jedoch, dass die Bewertung der Schutzbedürftigkeit auf gesellschaftlichen und politischen Entscheidungen beruht. Die naturwissenschaftliche Forschung kann zwar Daten zur Verbreitung und zur Individuenzahl einer Art liefern. Weiter können aufgrund der Biologie der Art Aussagen zur Mobilität, zur Verfügbarkeit von Lebensräumen und zu Reproduktionsraten getroffen werden. All diese populationsbiologischen bzw. ökologischen, d.h. naturwissenschaftlichen Daten ermöglichen lediglich Aussagen zur Folgenabschätzung. Es ist jedoch eine gesellschaftliche Entscheidung festzulegen, ab welcher Grenze die Art in eine bestimmte Kategorie der Roten Listen aufgenommen wird oder für wie viele Jahre und mit welcher Aussterbewahrschein- 
lichkeit im Rahmen einer Minimal-Viable-Populationanalyse zur Ermittlung minimal überlebensfähiger Populationsgrößen gerechnet wird ${ }^{54}$. Auf diese Weise enthalten die von den Naturwissenschaften bereitgestellten Informationen bereits gesellschaftliche, wissenschaftsoder naturschutzpolitische Wertungen.

Das zweite zentrale Kriterium ist die Schutzwürdigkeit. Sie beruht auf der Bewertung der Naturbestände durch den Menschen. Neben ökologischen Gesichtspunkten wird die Bewertung des Nutzens und Wertes der Natur für den Menschen stark von gesellschaftlichen und politischen Vorstellungen geprägt.

Die Zahlungsbereitschaftsanalyse kann auch hier wertvolle Beitrage und Argumentationshilfen leisten. Sie erweitert die naturwissenschaftlich-ökologische Argumentation um den Aspekt der Wertschätzung durch die lokale Bevölkerung. Wieder kann mittels Zahlungsbereitschaftsanalysen aufgezeigt werden, welche Bedeutung Landschaftsbild, Natur-, Kulturgüter und die Erholungsfunktion haben und ob es eine besondere Identifikation mit einem Naturausschnitt oder bestimmten Arten gibt (Anwendung analog der Anwendung im Bereich Eingriffsplanung).

\subsection{Fazit}

Erholungsfunktion und Freizeitwert, Unversehrtheit des Landschaftsbildes, Heimat, Lebensund Lernraum sind planungsrelevante Sachverhalte, die z. T. noch nicht ausreichend in den bisherigen Planungsverfahren berücksichtigt werden. Sollen diese menschenbezogenen Schutzgüter in die Umweltbewertung integriert werden, sind Zahlungsbereitschaftsanalysen das Mittel der Wahl. Eine Zahlungsbereitschaftsanalyse durchzuführen kostet Zeit und Geld. $\mathrm{Ob}$ dies sinnvoll und angemessen ist, muss im Einzellfall entschieden werden und darf die Verhältnismäßigkeit zwischen angestrebtem Nutzen und Aufwand nicht verletzen (siehe Kap. 8 hinsichtlich Verwaltungseffizienz, Kosteneffizienz, Verhältnismäßigkeit). Andererseits ist es fraglich, ob gesellschaftliche Belange adäquat durch einzelne Experten zu ermitteln sind. Dass die Bereitstellung einer guten Datengrundlage kosten- und zeitintensiv ist, kann man z. B. auch an den Tierartenerhebungen im Rahmen der Eingriffsregelung ablesen. Der gesetzliche Anspruch der Sicherung der Nutzungs-, Leistungs- und Funktionsfähigkeit des Naturhaushaltes ist nicht schnell und einfach zu erfüllen. Umso wichtiger ist die Wahl der

\footnotetext{
${ }^{54}$ Die Minimal-Viable-Populationanalyse berechnet die nötige Individuenstärke einer lokal begrenzten Tieroder Pflanzenpopulation, die notwendig ist, damit für einen bestimmten Zeithorizont eine ausreichend hohe Überlebenswahrscheinlichkeit gegeben ist. Was eine ausreichend hohe Überlebenswahrscheinlichkeit ist entzieht sich rein naturwissenschaftlichen Grundlagen sondern ist viel mehr eine Frage des gesellschaftlichen Diskurses. Hovestedt et al. (1992) setzen in ihren Berechnungen z. B. eine Überlebenswahrscheinlichkeit von $95 \%$ über 100 Jahre ein, Bräuer et al. (1999) rechnen mit einer Überlebenswahrscheinlichkeit von 90\% für einen Zeitraum von nur 10 Jahren.
} 
geeigneten Bewertungsmethoden.

Partizipative oder gesellschaftswissenschaftliche Methoden werden bisher nicht planungsbegleitend eingesetzt ${ }^{55}$. Auch wenn die Erfahrung aus formalen Beteiligungsverfahren vielfach negativ sind und die Beteiligung der Bevölkerung eher als Störfaktor denn als wertvoller Beitrag in Planungsverfahren angesehen wird, so kann doch festgestellt werden, dass eine gute Abstimmung der Pläne mit den Interessen der Bevölkerung die Ergebnisse des Planungsprozesses und deren Akzeptanz verbessern. Die Zahlungsbereitschaftsanalyse stellt hierfür ein sinnvolles Instrument dar. Die Bewertung wird auf Basis eines repräsentativen Ausschnitts der betroffenen Menschen durchgeführt. Die umfangreiche theoretische Ausarbeitung und vielfache Erprobung von Zahlungsbereitschaftsanalysen erlaubt die sachgemäße Anpassung des Untersuchungsdesigns an verschiedenartige Fragestellungen.

\subsection{Literatur}

Bayerisches Landesamt für Umweltschutz (Hg.) (1998): Kostendatei für Maßnahmen des Naturschutzes und der Landschaftspflege. Bearbeitet von M. Hundsdorfer. München

Bosch und Partner (1993): Faktische Grundlagen für die Ausgleichsabgabenregelung (Wiederherstellungskosten) i.A. der Bundesforschungsanstalt für Naturschutz und Ökologie (BFANL), F+E Vorhaben 10801151 , UFOPLAN 92, 1993

Bräuer I, W., Maibom, D. Matthies, T. Tscharntke (1999): Populationsgrößen und Aussterberisiko seltener kurzlebiger Pflanzenarten in Niedersachsen - Verhandlungen der Gesellschaft für Ökologie, Band 29, Ulm, S. 505-510

Breuer, W. (2000): Das Verhältnis der Prüfung von Projekten und Plänen nach $§ 19$ c BNatSchG zu UVP und Eingriffsreglung. Informationsdienst Naturschutz Niedersachsen 20 Nr.3, S.168-171

Gassner, E. (1995): Das Recht der Landschaft. Gesamtdarstellung für Bund und Länder. Radebeul: Naumann

Hessische Ausgleichsabgabenverordnung (1995): Ausgleichsabgabenverordnung (AAV) vom 09. Feb. 1995

Hessische Zusatzbewertung Landschaftsbild (1995): Zusatzbewertung Landschaftsbild als Bestandteil der Eingriffs- und Ausgleichsplanung. Verfahren gem. Anlage 1, Ziff. 2.2.1 der Ausgleichsabgabenverordnung (AAV) vom 09. Feb. 1995.

http://www.rpda.de/dezernate/eingriffsregelung/zusatzbewertung_ landschaftsbild/ (November 2003)

Hoppenstedt, A. (1996): Die Landschaftsplanung als Bewertungsgrundlage für die Eingriffsregelung Natur und Landschaft 71 (11), S. 485-488

Hovestadt, T., Roeser, J., Mühlenberg, M. (1991): Flächenbedarf von Tierpopulationen. Jühlich: Forschungszentrum Jühlich

Jessel, S. (1996): Die Eingriffsregelung zwischen naturwissenschaftlichem Anspruch und Anforderungen der Praxis. Laufener Seminarbeiträge 2/96, S. 9-16

Köhler, B., Preiß, A. (2000): Erfassung und Bewertung des Landschaftsbildes. In: Informationsdienst Naturschutz Niedersachsen 1/ 2000

Köppel, J., Feikert, U., Spandau, L., Strasser, H. (1998): Praxis der Eingriffsregelung. Stuttgart: Ulmer

Lambrecht, H. (1997): Richtwerte für Kompensationsmaßnahmen beim Bundesfernstraßenbau. Mitteilungen aus der NNA 2/97. S. 22-42

LANA (Hg.) (1996): Bearbeiter: Prof. Dr. H. Kiemstedt, S. Ott, M Mönnecke: Methodik der Eingriffsregelung

\footnotetext{
${ }^{55}$ Die spezifischen Anhörungs- und Erörterungsverfahren können nicht mit direkten Beteiligungsverfahren gleichgesetzt werden (ausführlich siehe hierzu Kap. 7 und Kap. 8).
} 
Teil 1, 2, 3. Gutachten zur Methodik der Ermittlung, Beschreibung und Bewertung von Eingriffen in Natur und Landschaft, zur Bemessung von Ausgleichs- und Ersatzmaßnahmen sowie von Ausgleichszahlungen. Teil 2: www.uvm.baden-wuerttemberg.de/nafaweb/ berichte/perw_02/perw2.htm, Teil 3: www.uvm.baden-wuerttemberg.de/nafaweb/berichte/perw_01/lana.htm. Kurzfassung: Kiemstedt, Mönnecke, Ott S. (1996): Methodik der Eingriffsregelung. Naturschutz und Landschaftsplanung 28 (9), S. $261-271$

Marggraf, R., Streb, S. (1997): Ökonomische Bewertung der natürlichen Umwelt. Heidelberg: Spektrum Akademischer Verlag

Ott, S. (1997): Methodik der Eingriffsregelung. NNA Berichte 10. Jg. Heft 3, S. 2-8

Penn-Bressel, G. (2001): Beeinträchtigungen der Erholungsfunktion durch Lärm und die Bezüge zur Eingriffsregelung. In: BfN (Hg.): Angewandte Landschaftsökologie Heft 44, S. 87-113

Plachter, H., Bernotat, D., Müssner, R., Riecken, U. (2002): Entwicklung und Festlegung von Methodenstandards im Naturschutz. Schriftenreihe für Landschaftspflege und Naturschutz Heft 70

Schweppe-Kraft, B. (1998) : Monetäre Bewertung von Biotopen und ihre Anwendung bei Eingriffen in Natur und Landschaft. Bonn-Bad Godesberg: Bundesamt für Naturschutz

Schemel, H.-J., Hartmann, G., Wedeking, K.-C. (1993): Methodik zur Entwicklung von Geldwertäquivalenten im Rahmen der Eingriffsregelung - Naturhaushalt - (Ausgleichsabgabe). Forschungsendbericht des Büros für Umweltforschung und Umweltplanung Dr. Schemel im Auftrage der Bundesforschungsanstalt für Naturschutz und Landschaftsökologie (BFANL). München. Kurzfassung veröffentlicht in Schemel, H.J./ G. Hartmann/ K.-C. Wedekind: Geldwertäquivalente für Beeinträchtigungen des Naturhaushalts - eine Methode zur Ermittlung der Ausgleichsabgabe bei Eingriffen, in: Natur und Landschaft, Heft 5, 1995, S. 213-220

SRU (2002): Sachverständigenrat für Umweltfragen: Für eine Stärkung und Neuorientierung des Naturschutzes. Sondergutachten 2002. Stuttgart: Metzler-Poeschel http://www.umweltrat.de/02gutach/downlo02/sonderg/1409852.pdf (Januar 2004)

Turner, K. (1991): Economics and Wetland Management. AMBIO Vol. 20(2), S. 59-63

United Nations Environment Programme (UNEP) (1995): Global Biodiversity Assessment. Cambridge (Cambridge University Press) 


\section{Kapitel 4 \\ Improving the institutional delivery of agri-environmental schemes via local action groups $^{56}$}

\subsection{Introduction}

Since 1992, the EU develops and supports measures for rural development and environmental protection within the common agricultural policy (CAP). Several times, the regulations have been improved with respect to economic efficiency and ecological efficacy but the EU always refused to finance manpower to implement the programmes. So, all work has to be done by the national administrations without additional payment. In the 2003 reform, the EU opened the regulation for financing so called local action groups. These local management concepts, successfully tested with the LEADER initiatives, will become a fundamental element within the new programme period of the rural development programme (Council Regulation (EC) No 1698/2005 of 20 September 2005 on support for rural development by the European Agricultural Fund for Rural Development, EAFRD).

The economic efficiency and ecological efficacy of agri-environmental schemes ${ }^{57}$ (AES) has often been discussed and questioned (see e.g. Kleijn et al., 2001; EU-Court of Auditors, 2000; Kleijn and Sutherland, 2003). It has also been stated that the ecological and economic effectiveness of different AES varies widely (Wilhelm, 2001; Marggraf, 2003; for a discussion on different interpretations of efficiency see e.g. SRU, 2002, Tz. 224-229). The EU tried to make the programmes for rural development (and within them the AES) more effective and efficient by implementing stricter control and evaluation instruments (Agri-GD, 2004; Wilhelm, 1999; Carey et al., 2003). With the promotion of local management within the LEADER concept and the integration of the LEADER concept into the rural development

\footnotetext{
${ }^{56}$ Published as Article Freese, J., H.-H. Steinmann (2006): Improving the institutional delivery of agrienvironmental schemes via local action groups. in B.C. Meyer (Ed.) 2006: Sustainable Land Use in Intensively used Agricultural Regions. Alterra report Landscape Europe, Wageningen, pp. 119-126.

${ }^{57}$ We use the term agri-environmental schemes as umbrella for all kind of measures often divided into agrienvironmental and contractual conservation measures.
} 
regulations (Agri-GD, 2005), the EU opens another path to improve effectiveness and efficiency of AES.

Hence, this paper aims to determine whether local management concepts provide increased potential to improve environmental and conservation goals in the agricultural landscape by a simultaneously improvement of acceptance, economic efficiency and ecological efficacy of agri-environmental schemes. We use a socio-economic approach to analyse the driving factors and main requirements to the AES and give a short insight into the way AES are implemented today. After reviewing different types of organisations for this local management, we point at the chances of local strategies. With the experience from the socioeconomic sphere of the "Bördeprojekt Wolfenbüttel" we demonstrate the positive impact of local management. Furthermore, we present factors of success for local AES-management strategies derived from the Bördeprojekt Wolfenbüttel and other environmental and nature conservation projects documented in the literature. Finally, we conclude with an outlook on options to implement a local management of the agri-environment and the idea of a nature conservation advisory service for farmers.

\subsection{Balancing AES: ecology, economics and farmers' acceptance}

With every new programme period the EU initiates the programming and implementation of $\mathrm{AES}^{58}$ in all German Länder (Land [sg.] - Länder [pl.]: in Germany also called Bundesländer, sometimes translated as federal states). Following the EU-regulations AES have to meet three needs:

1) AES are mostly directed towards farmers. Farmers participate voluntarily, so the programmes must be acceptable to them. This means that schemes must fit into their every day farming activities and must compensate costs and losses derived from programme participation.

2) AES aim to improve environmental and conservation issues, so the programmes must generate positive ecological effects. The measures must be ecologically effective.

3) AES are funded by money from EU, federal government and Länder sources, so the programmes have to be administrated effectively and the money must be used efficiently.

The EU sets only the framework for the AES (so called contextual guidance, see e.g. Willke,

\footnotetext{
${ }^{58}$ AES are a part of the EU rural development programmes, Programme period 1992-1999 Council Regulation (EC) 2078/1992; period 2000-2006: Council Regulation (EC) 1257/99; period 2007-2012: Council Regulation (EC) No 1698/2005 of 20 September 2005.
} 
1999, 39), and leaves the member states wide scope for the implementation. Here, we present two contrasting examples from German Länder how they have used this scope in the former period and characterise the resulting programmes (see also Wilson and Wilson, 2001, 201204).

A) The Proland-Programme of Lower Saxony can be seen as an example for the implementation of a limited set of agri-environmental measures uniform for the Land. In the former years, the farmers were able to choose between 4 and 10 measures ${ }^{59}$ within the AES. The aim of the administration was to keep the programme small and concise. The scheme has had no variations, top-ups, or restrictions concerning the spatial extensions and was not menu-driven.

B) Alternatively, a Land can provide a menu-driven programme with a large set of measures with multiple variability and top-ups. Within the Kulturlandschaftsprogramme of North Rhine-Westphalia or the MEKA in Baden-Wuerttemberg, for example the farmers can choose between at least 20 measures and variations. The countries or regions were allowed to define local aims and choose adequate measures, select variations, and set spatial restrictions to compile a local AES.

Generally, the Länder are responsible for the correct implementation of the EU-framework. In case of misuse or inadequate implementation, the administrations carry a (high) financial risk. The two strategies described above differ in their aims. In system A the efforts and costs of the administration for implementation, administration, and control are small and the programme is kept clearly arranged for the farmers. System B focuses more precisely on the ecological efficacy and practical requirements. It can be fitted into diverse local situations and covers many special conservation efforts. The drawback of system B is that the administration both at the Länder and at the local level requires more effort with the implementation, administration and control. Also, it is not easy for farmers to keep an overview about all possible combinations of the scheme.

But even the simpler Lower Saxony AES (System A) has been criticised for a lack of clarity The mid-term review of the programme shows that there is a lack of personal advice for the farmers. ML, 2003; Kap. 6, 23-24). The review positively highlights only one measure: the protection programme for rare arable weeds. It achieves the highest acceptance and most positive evaluation from the farmers. The farmers complimented the direct and personal advice given by the hosting employee of the administration.

\footnotetext{
${ }^{59}$ This included subsidies for organic farming. Not counted were specialised sub-programmes of the ProlandProgramme facilitating special conservation issues.
} 
Hence, the challenge to implement all three requirements - acceptance, ecological accuracy and economic efficiency - has not been successfully achieved. Thus, a lack of effectiveness and efficiency as well as a poor acceptance by the farmers is observed (Arzt et al., 2002; Deblitz, 1999; Jungcurt et al., 2004).

The economic efficiency aims at preventing windfall gains from paying overpriced measures $^{60}$ and at keeping the transaction costs of implementation, administration and control low. Economists normally try to apply this by installing a market. According to this, their proposal is to use calls for tenders and auction designs, and not to work with spatial or other limitation. If enough farmers participate at the call for tender and present different offers, the administration can choose those offers which are lowest in price. So, they hand out only relatively low grants and can contract a maximum area for reaching the protection target (examples for realising auctions see e.g. Cason and Gangadharan, 2004).

The ecological effectiveness demands to contract the most valuable and promising areas for conservation targets. This makes it necessary to have detailed information about the areas. To have or to develop locally adapted conservation targets is often connected with an inspection of fields. In most cases, this leads to a very limited and spatially fixed set of interesting areas from a conservation point of view. It is neither easy nor sure to get the appropriate areas under contract and this makes the measures expensive.

But no matter how the ecological and economic requirements are implemented, without farmers willing to contract, the best AES are worthless. So, the AES must be focused on acceptability ${ }^{61}$. Until now the aspect of acceptability is rarely put into account within the discussion on effectiveness of AES. Even though, Coleman et al. (1992, cited in Carey et al., 2003) argued that "policy measures which encourage positive attitudes to conservation will in long term be more effective than those do not." As a reason, they point out how the positive shift in the attitudes increases willingness to implement additionally voluntarily and unpaid measures. AES not designed to change the farmers' attitude will mostly be seen as temporary bribes, as Morris and Potter (1995) stated.

Regarding acceptance as a complex individual multi-step process (see also below and Prager, 2002 ; 12) it needs at least the same attention as the economic and ecological aspects of AES. For participation to occur, the farmer must have a positive attitude towards AES. Then, he must have access to the programme information. Also, he needs advice in this phase of

\footnotetext{
${ }^{60}$ There are several authors pointing to the tendency of AES in Germany to overcompensate farmers apart from the allowed 20\% incentive surplus (e.g. Ahrens et al., 2000).

${ }^{61}$ For a definition of acceptability see e.g. Prager, 2002, 6-29; Lehmann et al., 2005, 27-30 present an actual literature review about the discussion on acceptability of AES.
} 
checking the actual programme. The programme must fit into everyday farming activities. If all this is positively checked, the farmer still must apply for participation. Finally, if he has contracted, he will need support with the implementation and has to face the programme control. At every single step, one negative check will hinder him from participation. And even if he has contracted, every bad experience - a lack of support or negative experiences with the application and control process - will disincline him to participate the next time.

To meet all these demands, we propose to install regional management organisations for the agri-environment. A framework for the ecological and economic demands can be set at the Länder level, but the balancing between them can only be done locally. Therefore special local knowledge of both the ecological situation and conservation potentialities and the possibilities and requirements of the farmers is needed. Despite all possibilities of classical programme optimisation, substantial improvement can only be achieved by a regional representation and adaptation of AES with direct contact to the farmers.

In the following section, we highlight concepts for such regional organisations to foster the agri-environment and then suggest ways for the successful work of such institutions.

\subsection{Local management organisations for the agri-environment}

\subsubsection{Different organisation forms}

Local organisations for the management of the agri-environment and the local implementation, adaptation and promotion of AES help to close the gap between the clients, stakeholders and the administration. Nature conservationists, farmers and the local population should be integrated into the process, which is until now mostly only driven by the Länder administrations with consultations of the top organisations of public interest (see e.g. Freese and Rüffer, 2005).

The variety of organisations implementing the efforts of local management is enormous (Blum et al., 2000) and they can generally be referred to as regional intermediate organisations $^{62}$ or local action groups. Examples are:

- Landcare associations (DVL, 2005; Speer, 2000),

- Project born advisory boards, composed of local experts and stakeholders, like the "advisory board for the agri-environment" in the district of Northeim (Bertke et al., 2005; Hespelt, 2005, 35-98),

\footnotetext{
${ }^{62}$ See e.g. Streeck, 1999, they are also called "encompassing" (Olson, 1982) or "corporatistic" (Schmitter, 1979) organisations.
} 
- The integrated area management in the water protection area "Fuhrberger Feld" (Haaren et al., 2005), a cooperation between farmers, scientists and water supply companies.

- The Agrarumweltforum Grano (Arzt et al., 2002), a round table concept in Brandenburg.

- Nature conservation and biological stations, driven by private conservation organisations but supported and assigned by Länder (e.g. for North Rhine Westphalia see Neiss, 2001),

- The initiatives of LEADER (LEADER, 2005) and Region Aktiv (BMVEL, 2005; Brocks and Weiß, 2004; Knickel et al., 2004) implemented the ideas of local management even if they not always deal with the agri-environment.

\subsubsection{Experiences in a local project: The "Bördeprojekt Wolfenbüttel"}

In the Börde region of the Wolfenbüttel District arable land is very productive ${ }^{63}$. Land is valuable and farms are modern and thriving. This is a situation where nature conservation programmes are not very competitive against production incomes and farmers tend to use their full acreage for intensive production, so except the casual participation in a conservation tillage measure, farmers in the Börde region of Wolfenbüttel did not participate at all in AES. However, also in highly productive regions it is necessary to fulfil nature conservation standards, e.g. to develop a net of extensive margins to ensure a positive nature conservation impact.

Starting in 2003, the Bördeprojekt aims at analysing the social, political, and economic driving factors in the field of agri-environmental protection and developing AES adjusted to this intensively used region and to motivate the farmers to participate. Before 2003, the Lower Saxonian AES was mainly directed towards grassland and marginal sites. On the one hand, facing the modulation of direct EU-payments farmers and agricultural authorities became increasingly interested in measures for agricultural productive regions. On the other hand, the Börde regions lack semi-natural and natural habitats due to the long-lasting intensive agricultural usage. This was the starting point for the project funded by the Deutsche Bundesstiftung Umwelt (DBU) and supported by the Ministry of Agriculture, Lower Saxony. Main aspects of the accompanying scientific research were institutional, political and economic aspects.

\footnotetext{
${ }^{63}$ Börde in Germany is referring to intensively used agricultural landscapes. Soils are mainly derived from loess. The Börde regions offer excellent conditions for arable farming. The Börde region of Wolfenbüttel (in the eastern part of Lower Saxony, Germany) is mainly used as agricultural land, which covers $69 \%\left(500 \mathrm{~km}^{2}\right)$ of the Wolfenbüttel District. There are approximately 350 farms laying within the district
} 
Despite the above-mentioned critics on the ecological effectiveness a positive environmental and ecological impact of extensification measures in the Börde region was assumed and not investigated.

The project was locally installed at the existing Landcare Association. The first step of the project was to establish an advisory board consisting of the local stakeholders like farmers, farmers union, conservationists, district administrations, agricultural chamber and the local agrarian extension services, and additional experts from the Ministry of Agriculture and the ecological advisory body of Lower Saxony. This board gathered reasons that prevented farmers from participating in AES. These results were accompanied by a census. A questionnaire was sent to all full time farmers $(n=320)$ in the Wolfenbüttel District with at least 15 ha farmland. From the returned 79 questionnaires it became apparent that in addition to pure economic reasons, operational and structural reasons led farmers to refuse agri-environmental schemes. The 5 year contracting period of AES is one of the major obstacles. In a time where European agricultural policy changes significantly farmers in the Wolfenbüttel District try to avoid long term contractual binding to AES. Another obstacle is the large number of schemes and programmes with different rules and regulations. Even if there were adequate measures, the variety of schemes, lacking sources of advice and lacking motivation hinder farmers to participate $^{64}$.

The project started with the assumption that improper schemes and measures are not the bottleneck, but a lack of farmers' information and motivation. The Bördeprojekt installed a local management at the Landcare Association Wolfenbüttel. In cooperation with the Centre for Agriculture and the Environment (ZLU) farmers, local authorities, and nature conservation associations were invited to define local goals and adequate measures. Information on agri-environmental schemes was gathered and farmers were encouraged to participate. The project also assisted during the process of application and implementation of the measures.

As central measure, the establishment of flowering field margin strips ( 3 to $25 \mathrm{~m}$ width, no use of fertiliser and plant protection agents and no mowing allowed until the end of the vegetation period) across the Wolfenbüttel District was initiated ${ }^{65}$. Different sources of funding like impact-regulation, a river margin programme, a special Lower Saxonian agrienvironmental scheme for the Wolfenbüttel District and project-funding were used. The experiences were recorded and discussed with the partners and actors from agriculture,

\footnotetext{
${ }^{64}$ Detailed analysis see: Freese and Steinmann $(2005,15-28)$.

${ }^{65}$ Other used and aimed at measures are the establishment of set-asides, grass strips, meadow and Hedges within the Börde region. But according to the experts' opinion and the survey, the flowering field margin strips were the most promising and favoured measure.
} 
conservation, and administration. This influenced the development of a Lower Saxony wide implementation of an additional agri-environmental scheme in 2004. Now, farmers can be paid for extensivated flowering field margin strips. It is not allowed to use the strips for production purpose, to apply fertiliser or plant protection agents, but the farmers have to sow flowering plants.

\section{Project results}

The process of developing and testing measures in a local context together with the invested manpower and the involvement of the relevant local partners increased the interest and acceptance among farmers, nature conservationalists and authorities.

As a result, in 2005 more than 170 ha or approximately $140 \mathrm{~km}$ of flowering field margin strips were sown in the district of Wolfenbüttel. This is a remarkable success and shows that it is possible to achieve nature conservation goals in highly productive areas, if a local organisation drives the development of the agri-environment.

Additionally a straightforward cooperation of local actors was established. At the beginning the farmers and their organisations strictly opposed the central funding mechanism of the AES. This so called "modulation" transfers money from the first pillar of the European common agricultural policy (market support e.g. subsidies for farmers) to the second pillar (rural development). The farmers get the money from the second pillar only by contracting to additional activities like AES. But after a while the farmers supported the measures, promoted the participation and finally called for prolongation of the measure.

\subsection{Discussing factors of success for AES}

\subsubsection{Usage of existing networks}

Networks of farmers and their organisations as well as the structures of nature conservation differ between regions. Intermediate organisations for the improvement of the (agri-) environment are not widely established. Building up new structures between the members of nature conservation unions and farmers can easily take years. Forming a new structure between stakeholders in the field of agri-environmental improvement requires an impartial and well-balanced process. To avoid barriers between groups, it is favoured to set up a project or organisation that consists of all relevant local stakeholders.

As a frequently successful example we present the also in the Bördeprojekt Wolfenbüttel used concept of the German Landcare Associations (DVL, 2005; Speer, 2000). Here the local stakeholders such as members of the administration, the farmers, and the members of the 
conservation unions constitute an association together. The three parties are equally represented in the steering committee. The Landcare Association (LA) is open to both further individual and constitutional members.

In the Wolfenbüttel Landcare Association farmers, the district administration and members of conservation unions worked already together for 7 years in several small nature protection projects. The Bördeprojekt benefited from the existing cooperative climate and was able to use the structures and contacts of the groups within the LA.

\subsubsection{A dualistic partnership with a strong "public to private" ele- ment}

The specific advantage of LA is that there is an organisational frame for the stakeholders to communicate and work together at a limited topic. Hence, the members work goal- oriented, and the dualistic partnership raises their chances of success. Dualistic partnership ${ }^{66}$ means that the partnership has not only a 'private to private' element between private stakeholders like farmers, conservationalists, landowners and hunters, but also a strong 'private to public' element. This 'private to public' element is represented by the regular participation of members of the administration or politicians. The close connection to the decision-making sphere of administration and politics motivated the private stakeholders to participate and to invest time in the Bördeprojekt. Motivated by the chance to get results directly to the implementing administration, all stakeholders had an additional interest in participating and finding presentable solutions. The chance of participation in the formation of AES stimulated the stakeholders for a conceptual and constructive cooperation.

\subsubsection{Dedicated management}

Especially in the field of environmental and nature conservation, direct motivation of people in our case, the farmers - is the key to success (see e.g. Brendle, 1999). Although surveys show a general awareness for the importance of nature conservation (BMU, 2002; BMU, 2004), practical experience shows that people neither see their own responsibility nor their possibilities and calls for action to improve the environmental quality ${ }^{67}$. A variety of attitudes and phenomena hinders people to contribute to nature conservation issues in their every day life: e.g. the so-called "Not-in-my-Backyard-phenomena", the "what-for-heavens-sake-can-I-

\footnotetext{
${ }^{66}$ The dualistic organisation principle is one element of new governance strategies often summarised under the topic of "good governance". With integrating well-governed (private) networks into a lager public environment they aim at making the policy leaner while simultaneously securing supra-individualistic rationality (Elsner, 2000, $435 \mathrm{ff}$, Bogason, 2000, $76 \mathrm{f}$ ).

${ }^{67}$ The problem is discussed under the term "deficits of acceptance for nature conservation" (see e.g. SRU, 2002, Tz. 77-93; Schuster, 2003).
} 
do-within-these-worldwide-problems" attitude (see e.g. Ott, 2002) and the individual cognition, that the environment in the local context is in good shape, but that massive problems are found in the world wide and far away ecosystems (BMU, 2002, 36).

To conquer this barrier, local management is required. A known and trusted organisation like the LA in the Bördeprojekt is the background for a local action group or a promoting team for the agri-environment. Without people dedicated to the topic and the common aims, an organisation is inanimate and lifeless. On the one hand, the manager has to keep the organisation alive. This means he has to motivate the stakeholders to contribute to the organisation, to discuss and develop common aims and projects. On the other hand, the manager is the driving force for the implementation of the organisation's aims. He must appoint the local calls for action, possible resolutions and the contribution everybody can bring. Together with the following factor of success - the process competence - this must be done without a moralising undertone and in an adequate and friendly way. Practical demonstrations and an intensive dialogue are a very good way to do so. The manager must listen to the farmers and their concerns. Only if they are taken serious, the manager has a chance to motivate them. To get in contact with the farmers requires good connection and a trusting appearance. In the dialogue it is important to find good reasons for the farmers to get involved (find win-win situations). But this is just the first step. Afterwards the manager has to gather information continuously and to disseminate them across the farmers. He must support farmers with the application and practical questions.

In the Bördeprojekt this task was carried out by the secretary of the LA Wolfenbüttel in cooperation with the project partners from the ZLU, the farmers union and the Agricultural Chamber. The growing number of farmers, contacting this persons in general questions, concerned with environmental and conservation issues is a good indicator for the success of the work.

\subsubsection{Process competence}

As mentioned above, the people behind the organisation have to fulfil a complex task. They have to bring together the stakeholders and motivate them to participate in the process of finding common aims and strategies and to implement them. Often it is important to balance between the stakeholders, to find areas where cooperation is possible and to exclude fields touching taboo topics.

They also have to organise and to steer the processes and to coordinate the activities of the stakeholders. Finally, it is their task to get farmers involved in environmental protection and 
nature conservation. This again is a multiple step process (see e.g. Prager, 2002; 12):

1. get farmers interested in AES

2. motivate them to collect information about measures and funding regulation and check their possibilities to implement AES on their farm

3. motivate them to participate and test the programme

4. motivate them to maintain the measures

For every step an adapted action is needed. Additionally, every farmer must be addressed individually. The manager cannot follow a straight roadmap. Instead, he has to adapt it to the people and the appearing problems.

This illustrates what is meant with the term 'process competence'. Building up trust and cooperation is always a long, fragile and multi-step process. Loss of confidence and withdrawal of willingness to cooperate can be caused by one wrong word or one rash action.

The process competence within the Bördeprojekt was provided by the experience the project staff. Especially the experiences of the secretary of the LA as secretary of the regional farmers union and the partner from the agricultural chamber concerned with environmental questions allowed the project to adapt the strategy to the local particularities.

\subsubsection{Flexibility matters}

To support these organisational and personal factors of success, flexibility is a crucial factor (Brendle, 1999; Hampicke, 2001). The managing organisation must not only possess flexibility in its strategies, but the AES and the measures must also offer flexibility, in order to adapt to individual and special situations and demands. The variability of funding including free disposable project-funding and the possibilities to use structures or consult the specialists e.g. within the agricultural chamber, the farmers union and the administration to answer questions or solve problems were central elements of the flexibility within the Bördeprojekt.

\subsection{Conclusions}

Taking into account the presented findings from the ecological, economic and acceptance demands it becomes evident that the efficiency and efficacy of the AES can be improved best by installing and supporting an organisation for the local management of the agri-environment instead of improving the regulations only in the common way at Länder level ${ }^{68}$. This is confirmed by the local experiences in the Wolfenbüttel District and was also reported by other studies (e.g. LEADER, 1997; Geißendörfer et al., 1998; Geißendörfer and Seibert, 2004). The

\footnotetext{
${ }^{68}$ For an introduction into the debate on centralisation versus decentralisation with lots of bibliographic hints see e.g. Lehmann et al., 2005, 20-23; Eggers, 2005, or Feindt and Newig, 2005, 17-23.
} 
organisations, pillared on the participation of the relevant stakeholders, should be enabled to design local AES or to adapt measures to local specialities and requirements and the local discussion processes.

For cross compliance rules ${ }^{69}$ as well as general environmental aspects, the need of individual advisory services for farmers is accepted within the midterm-review reform a funding possibility is implemented into the EU regulations. For the more specific implementation of nature conservation aspects, the farmers lack a service of individual advice and a financial stimulus to inform themselves about voluntary nature conservation measures on their farm. In Germany an initiative has recently begun to build up a network for nature conservation advisory services for farms ${ }^{70}$ (Keufer and van Elsen, 2002). The future task is to fit existing local structures concerned with agri-environmental topics to the European efforts of a cooperative rural development to form a local management for the agri-environment.

\subsection{References}

Agri-GD - Agriculture Directorate-General, 2004. Common Indicators For Monitoring Rural Development Programming 2000-2006. Commission Working Document D/761 FINAL: 01.2005.

http://europa.eu.int/comm/agriculture/rur/eval/guide_en.pdf (10.1.06)

Agri-GD, 2005. Strategic Guidelines for Rural Development. Press release of the EU, 5.7.2005. http://europa.eu.int/comm/agriculture/capreform/rdguidelines/index_en.htm (10.1.2006)

Ahrens, H., Lippert, C., Rittershofer, M. 2000. Überlegungen zu Umwelt- und Einkommenswirkungen von Agrarumweltprogrammen nach VO (EWG) Nr. 2078/92 in der Landwirtschaft. Agrarwirtschaft 49 (2), 99-115 (in German).

Arzt, K., Baranek, E., Berg, C., Hagedorn, K., et al., 2002. Projektbereich 1: Dezentrale Bewertungs- und Koordinationsmechanismen. In: Müller, K., V. Toussaint, H.-R. Bork, K. Hagedorn, J., Kern, U.J., Nagel, J., et al. (Eds.) 2002. Nachhaltigkeit und Landschaftsnutzung: Neue Wege kooperativen Handelns. Margraf, Weikersheim, pp. 29- 96 (in German).

Bertke, E., Hespelt, S.-K., Marggraf, R., 2005.Ein regionaler Beirat als partizipatives Gremium in der AgrarUmweltpolitik. in: Feindt, P., Newig, J. (Hrsg.): Partizipation, Öffentlichkeitsbeteiligung, Nachhaltigkeit. Metropolis, Marburg, pp.281-298 (in German).

Blum, B., Borggräfe, K., Kölsch, O., Lucker, T., 2000. Partizipationsmodelle in der Kulturlandschaft: Analyse von erfolgsfördernden Faktoren in 13 Regionalentwicklungsprojekten. Naturschutz und Landschaftsplanung 32 (11), 340-346 (in German, with English abstract).

BMU (Federal Ministry for the environment), 2002.Umweltbewusstsein in Deutschland 2002, Bonn. http://www.empirische-paedagogik.de/ub2002neu/download/umweltbewusstsein2002.pdf (6.1.2006) (in German).

BMU, 2004: Umweltbewusstsein in Deutschland 2004, Bonn. http://www.bmu.de/files/broschueren/faltblaetter/application/pdf/umweltbewusstsein2004.pdf (3.1.2006) (in German).

BMVEL (Federal Ministry for Agriculture), 2005: Region Aktiv - Land gestaltet Zukunft. http://www.novainstitut.de/modellregionen (20.12.2005) (in German).

Bogason, P., 2000. Public Policy and Local Government. Institutions in the Postmodern Society. E. Elger,

\footnotetext{
${ }^{69}$ Cross compliance mean the bondage of the EU direct payments for farmers to their adherence to regulations concerned with environmental and nature protection, quality management, consumer protection and animal welfare (Council Regulation (EC) 1782/2003).

${ }^{70}$ see also http://www.naturschutzberatung.info
} 
Cheltenhham.

Brendle, U., 1999. Musterlösungen im Naturschutz - Politische Bausteine für erfolgreiches Handeln. Münster: Landwirtschaftverlag (in German).

Brocks S., Weiß, K., 2004. Das Instrument des Wettbewerbs als Impulsgeber für die Regionale Zusammenarbeit. Berichte über Landwirtschaft 82 (1) 5-25 (in German, with English abstract).

Carey, P.D., Short, C., Morris, C., Hunt, J., Priscott, A. et al. 2003. The multi-disciplinary evaluation of a national agri-environment scheme, Journal of Environmental Management, Volume 69 (1) 71-91.

Cason, T.N., Gangadharan, L., 2004. Auction Design for Voluntary Conservation Programs. American Journal of Agricultural Economics 86, 1211-1217.

Coleman, D., Crabtree, B., Froud, J., O'Carroll, L., 1992. Comparative Effectiveness of Conservation Mechanisms. Department of Agricultural Economics, Manchester University, Manchester.

Deblitz, C., 1999. Vergleichende Analyse der Ausgestaltung und Inanspruchnahme der Agrarumweltprogramme zur Umsetzung der VO (EWG) 2078/92 in ausgewählten Mitgliedsstaaten der EU. Landbauforschung Völkenrode, Sonderheft 195 (in German).

DVL (German Landcare Assosiation), 2005: Landcare Associations - an alliance for Nature. http://www.lpv.de/fileadmin/user_upload/data_files/International/flyer_eng_050331.pdf (20.12.2005).

Eggers, J., 2005. Institutionelle Hemmnisse einer dezentralen Ausgestaltung der Agrarumweltmaßnahmen in der Europäischen Agrarpolitik. Shaker, Aachen (in German).

Elsner, W., 2000. An Industrial policy agenda 2000 and beyond. In: Elsner, Groenewegen (Ed.). Industrial Policies after 2000. Kluwer, Boston, Dordrecht, London.

EU-Court of Auditors 2000. Special Report No 14/2000 on "Greening the CAP" together with the Commission's replies. Official Journal of the European Communities, Volume 43. http://www.eca.eu.int/audit_reports/special_reports/docs/2000/rs14_00en.pdf (11.1.06).

Feindt, P.H., Newig, J., 2005. Politische Ökonomie von Partizipation und Öffentlichkeitsbeteiligung im Nachhaltigkeitskontext. In: Feindt, P.H., Newig, J. (Eds.) 2005: Partizipation, Öffentlichkeitsbeteiligung, Nachhaltigkeit. Marburg, Metropolis, pp.9-42 (in German).

Freese, J., Rüffer, C., 2005.Kooperativer Naturschutz in der Kulturlandschaft. In: Feindt, P.H., Newig, J. (Eds.). Partizipation, Öffentlichkeitsbeteiligung, Nachhaltigkeit. Marburg, Metropolis, pp.257-280 (in German).

Freese, J., Steinmann H.-H., 2005. Ergebnisse des Projektes „Randstreifen als Strukturelemente in der intensiv genutzten Agrarlandschaft Wolfenbüttels“. Diskussionsbeitrag 0503 des Inst. f. Agrarökonomie, Universität Göttingen, Göttingen (in German)

http://wwwuser.gwdg.de/ uaao/margg/discussionpaper0503.pdf (13.1.06).

Geißendörfer, M., Seibert, O., 2004. Ex post-Evaluierung der Gemeinschaftsinitiative LEADER II. Berichte über Landwirtschaft 82 (2), 188-224 (in German, with English abstract).

Geißendörfer, M., Seibert, O., Meyer, H. von, 1998. Ex post-Evaluierung der Gemeinschaftsinitiative LEADER I in Deutschland. Berichte über Landwirtschaft 76, 540-579 (in German, with English abstract).

Haaren, C. von, Brenken, H., Hachmann, R., 2005. Integriertes Gebietsmanagement Fuhrberger Feld. Naturschutz und Landschaftspflege 37 (9) p.261-268 (in German, with English abstract).

Hampicke, U., 2001. Agrarumweltprogramme und Vorschläge für ihre Weiterentwicklung. In Osterburg, B., Nieberg, H. (Eds). Agrarumweltprogramme - Konzepte, Entwicklungen, künftige Ausgestaltung. Landbauforschung Völkenrode, Sonderheft 231: 97-109 (in German).

Hespelt, S.K., 2005. Regionalisierung von Agrarumweltmaßnahmen: dargestellt am Beispiel der transdisziplinären Entwicklung eines ergebnisorientierten, regional verankerten Honorierungssystems für ökologische Leistungen der Landwirtschaft unter besonderer Berücksichtigung eines regionalen Gremiums. Ibidem, Stuttgart (in German).

Jungcurt, S., Laschewskiz L., Schleyer C., 2004. Löst Geld Nutzungskonflikte? Zur Steuerungsproblematik von Förderprogrammen im Agrarumweltbereich. http:/www.agrar.huberlin.de/wisola/fg/ress/web/Studie_Spreewald.pdf (4.1.2006) (in German).

Keufer, E., van Elsen, T., 2002. Naturschutzberatung für die Landwirtschaft. Ergebnisse einer Umfrage bei Bioland-Landwirten und Ansätze zur Institutionalisierung in Niedersachsen. Naturschutz und Landschaftsplanung 10: 293-299 (in German, with English abstract). 
Kleijn, D., Brendse, F., Smit, R., Gilissen, N., 2001. Agri-environment schemes do not effectively protect biodiversity in Dutch agricultural landscape. Nature 413: 723-725.

Kleijn, D., Sutherland, W.J., 2003. How effective are agri-environment schemes in conserving and promoting biodiversity? Journal of Applied Ecology 40: 947-969.

Knickel, K., Siebert, R., Ganzert, C., Dosch, A., Peter, S., Derichs S., 2004. Ergebnisse der Begleitforschung 2002-2003: Abschlussbericht. Wissenschaftliche Begleitforschung des Pilotprojektes „Regionen Aktiv Land gestaltet Zukunft".

http://www.nova-institut.de/ra-attach/7867/RA_BF_fin.pdf (4.1.2006) (in German).

LEADER 1997. Organising local partnerships. Innovation in rural areas, Notebook no 2. LEADER European Observatory.

http://europa.eu.int/comm/archives/leader2/rural-en/biblio/partner/contents.htm (3.1.2006).

LEADER 2005. Deutsche Vernetzungsstelle Leader+. http://www.leaderplus.de (20.12.2005) (in German).

Lehmann, P., Schleyer, C., Wüstemann, H., Drechsler, M., Hagedorn, K., Wätzold F., 2005. Promoting the Multifunctionality of Agriculture, Forestry, and Rural Areas - Design and Implementation of Public Policies in Germany. UFZ-Diskussionspapier 12/2005. http://www.ufz.de/data/Disk_Papiere_2005-122648.pdf (4.1.2006).

Marggraf, R., 2003. Comparative Assessment of Agri-environment Programmes in Federal States of Germany. Agriculture, Ecosystems and Environment 98: 507-516.

ML - Ministry of Agriculture, Lower Saxony, 2003. Halbzeitbewertung von Proland Niedersachsen. Braunschweig. http://www1.ml.niedersachsen.de/proland/Aktuelles.htm (21.12.05) (in German with English abstract).

Morris, C., Potter, C., 1995. Recruiting the new conservationists: farmers' adoption of agri-environmental schemes in the UK. Journal of Rural Studies 11: 51-63.

Neiss, T., 2001. Biologische Stationen in NRW: Gütesiegel der Naturschutzpolitik. LÖBF-Mitteilungen Nr. 3/2001: 8-12 (in German).

Olson, M., 1982. The Rise and Decline of Nations: Economic Growth, Stagflation, and Social Rigidities. Yale University Press, New Haven.

Ott, K., 2002. Akzeptanzdefizite im Naturschutz, in: DRL (Hrsg.): Die veschleppte Nachhaltigkeit: frühe Forderungen - aktuelle Akzeptanz, Heft 74. pp.75-81 (in German).

Prager, K., 2002. Akzeptanz von Maßnahmen zur Umsetzung einer umweltschonenden Landbewirtschaftung bei Landwirten und Beratern in Brandenburg. Margraf, Weikersheim (in German).

Schmitter, P.C., 1979. Still the Century of Corporatism? In: Schmitter and Lehmbruch (eds.), Trends Towards Corporatist Intermediation. Sage, London and Beverly Hills, pp. 7-52.

Schuster, K., 2003. Image und Akzeptanz von Naturschutz in der Gesellschaft. In DRL (Ed). Naturschutz in Deutschland - eine Erfolgsstory? 12/2003, pp. 80-88.

Speer, D., 2000. Naturschutzpraxis im Beteiligungsmodell: Landschaftspflegeverbände. In: Schweppe-Kraft, Burkhard (Ed.). Innovativer Naturschutz: Partizipative und marktwirtschaftliche Instrumente. Bundesamt für Naturschutz, Bonn-Bad Godesberg, pp.73-86 (in German).

SRU (The German Advisory Council on the Environment) 2002. Sondergutachten 2002. Für eine Stärkung und Neuorientierung des Naturschutzes. (Towards Strengthening and Reorienting Nature and Landscape Conservation) Metzler-Poeschel, Stuttgart (in German).

http://www.umweltrat.de/02gutach/downlo02/sonderg/SG_Naturschutz_2002.pdf (6.1.2006).

Abbreviated English version of the special report

http://www.umweltrat.de/english/edownloa/specrepo/SG_Towards_Strengthening_2002_summary.pdf (10.1.06).

Streeck, W., 1999.Überlegungen zur Rolle von intermediären Organisationen in sich ändernden Umwelten. In: Streeck, W., 1999. Korporatismus in Deutschland. Campus, Frankfurt/ Main, N.Y., pp.223-250 (in German).

Wilhelm, J., 2001. Ergebnisse einer Ausgaben-Wirksamkeits-Analse der Agrarumweltprogramme. In Osterburg, B., Nieberg, H., (eds.). Agrarumweltprogramme - Konzepte, Entwicklungen, künftige Ausgestaltung. Landbauforschung Völkenrode, Sonderheft 231, 25-32 (in German).

Wilhelm, J. 1999. Ökologische und ökonomische Bewertung von Agrarumweltprogrammen - Delphi-Studie, 
Kosten-Wirksamkeits-Analyse und Nutzen-Kosten-Betrachtung. Europäische Hochschulschriften V/2542, Peter Lang, Frankfurt/ Main (in German).

Willke, H., 1999: Systemtheorie II: Interventionstheorie. 3. Aufl., Stuttgart, UTB (in German).

Wilson, G.A., Wilson, O.J., 2001.German agriculture in transition: society, policies and environment in a changing Europe. Palgrave, Basingstoke. 


\section{Kapitel 5}

\section{Stakeholder involvement in agri-environmental policy making - Success factors for participatory approaches from two German case studies $^{71}$}

\subsection{Introduction}

The recent reform of the rural development policy (EAFRD, Council Regulation (EC) No 1698/2005 of 20 September 2005) of the European Union (EU) requires the Member States to strengthen the involvement of stakeholders in the development and implementation of rural development programmes. The EAFRD-Regulation demands a 'partnership' to be involved in preparation, implementation, monitoring and evaluation of rural development programmes (Article 6). Additionally the rural development programme is endorsed by the LEADER approach of local action groups as local public-private partnerships (Art $61 \mathrm{ff}$ ). Since the European regulations for the development of rural areas work as contextual guidance, they leave the Member States an enormous leeway in the implementation process. Additionally, regarding the EU-principle of subsidiarity ${ }^{72}$ the aims and ideas of the local people and stakeholders need to be integrated into the process of both programming and implementing the rural development programmes in the Member States.

The regulation consists of 4 axes:

- Improving the competitiveness of the agricultural and forestry sector;

- Improving the environment and the countryside;

- Diversification of the rural economy and Quality of Life in rural areas;

- Continuous support for the community initiative LEADER.

In dealing with aspects of improving the agricultural environment, this paper focuses on axis 2. One of the main instruments for rural development is the agri-environmental schemes (AES). Together with the new nature conservation strategy of cooperative implementation of

\footnotetext{
${ }^{71}$ Article wirtten together with Katrin Prager (Berlin). Accepted with substantial revision at the Journal of Environmental Management.

72 'Subsidiarity' is the concept that matters should be handled by the smallest (or the lowest) competent authority, a central authority should only perform those tasks which cannot be performed effectively at a more immediate or local level. Subsidiarity was established in EU law by the Treaty of Maastricht (1992).
} 
conservation (see e.g. Wilson and Wilson, 2001, pp. 198f), AES represent a strong resource for environmental and nature conservation and an ideal study area for stakeholder involvement $^{73}$.

Since the environment and nature are resources of public use and benefit (public goods) AES should act horizontally through different sectors (conservation, water protection, countryside development). Due to their voluntary nature, agri-environmental schemes depend on farmers' acceptance and participation for their implementation. Moreover, not only farmers but also the interests of a larger set of rural stakeholders have to be considered. The growing focus on agriculture as one of the main activities shaping the landscape as well as the discussion of multi-functionality make it important to involve traditional bodies, agrarian interest groups, non-agrarian stakeholders and regional stakeholders. However, authorities responsible for programme coordination are lacking the knowledge of whom to involve when and for which purpose. Or as in the words of the drafters of the Regulation: How should "the conditions for a broad and effective involvement of all appropriate bodies, in accordance with national rules and practices" be achieved? A single participatory approach may not be adequate for every policy-making level. One fixed tool may not cater for the demands of the various stakeholders affected by rural issues. Existing participatory approaches will need to be tested and adapted to explore the necessary expenditures and coordination efforts. The question is how the 'partnerships' can effectively entail positive impacts on information availability, process transparency and "better" (more satisfying) outcomes.

Cooperation and voluntary participation are crucial elements not only in agri-environmental programme development but also for the effectiveness of the resulting measures. In this context the paper presents two studies concerned with stakeholder involvement carried out in two Länder ${ }^{74}$ of Germany: Lower Saxony and Sachsen-Anhalt. The research is targeted at the involvement of stakeholders in the programming and implementation of AES at the local and state level. In Sachsen-Anhalt a decision-support system based on linear programming (referred to in the rest of the paper as 'mathematical model') was used to optimise the programme planning and budget allocation at state level, accompanied by facilitated communication processes. In Lower Saxony a local partnership (or in $\mathrm{LEADER}^{75}$ terms a local action group) developed and tested a selected agri-environmental measure, which was subsequently inserted into the Lower Saxony AES.

\footnotetext{
${ }^{73}$ For a more general introduction to institution building and stakeholder involvement in environmental issues see e.g. Renn et al. (1995), Feindt and Newig (2005), Ostrom (1994) pp. 319-329.

${ }_{74}^{74}$ Land [sg.] - Länder [pl.]: in Germany also called Bundesländer, sometimes translated as federal states.

${ }^{75}$ LEADER is one of four initiatives financed by EU structural funds and is designed to help rural actors consider the long-term potential of their local region.
} 
The paper starts by outlining the process and responsibilities of agri-environmental policy focusing on Germany's Länder level. The differing and partly conflicting interests associated with this process are discussed. The case studies are subsequently presented with their characteristics and history tabulated to allow for a quick overview. Finally, the findings of the two case studies are discussed with respect to premises and necessary preparation, aspects of structuring and facilitating the participatory process. The problems encountered during the projects are contrasted with the benefits of the approaches before concluding with recommendations.

A note on terminology: 'Implementation' can take place on different levels, e.g. Member States implement EU regulations (adopting them in national legislation), local level administrations are responsible for implementing Länder schemes (deliver schemes to farmers) or farmers can implement measures (carry out prescription, conservation or land management activities). Since the focus of the paper is on case studies in selected federal states, state government will be referred to as upper (policy making) level, while 'local' refers to any level below the state level. If 'regions' are mentioned in this paper the term relates to a part of a federal state, e.g. local agriculture agencies (Landwirtschaftsämter) are responsible for a region. The lower level as the policy implementing level may refer to local administration as well as individual farmers, conservationists, associations etc.

\subsection{Agri-environmental programming}

\subsubsection{Process and responsibilities}

In Germany it is the responsibility of the Länder to implement the Council regulation on support for rural development (EAFRD). Funds from this so called second pillar of the common agricultural policy (CAP) are among the most important sources for the development of rural regions. For example, in Lower Saxony the budget for 2000-2006 was more than 1.1 billion Euros with approximately 50\% coming from the EU.

There are three levels of administration and policy making in Germany. The federal level (Bund), the state level (Länder) and the local level (municipalities). Regarding agrienvironmental policy making, the European Commission (which we are not going to investigate) and the Länder are the major players. Although the federal level does play a role by providing additional funds, the Länder are responsible for regional and structural planning and agri-environmental policy (Grafen and Schramek, 2000). With the implementation power delegated to the individual Länder, each of them has developed their own set of schemes 
adapted to their specific environmental problems ${ }^{76}$.

Programme planning is a major task of the Länder ministries. In most German Länder the ministry for agriculture leads the compilation of the Rural Development Programme, including the agri-environmental programmes as a part or sub-programme. While in Sachsen-Anhalt the agriculture and environment sectors are placed within one ministry, Lower Saxony has two separate ministries for these functions. In the latter case therefore, a number of conservation measures are administrated by the environment ministry.

After the EU has adopted a new regulation the Member States are obliged to start their planning process. Any funds the EU spends within the second pillar of the CAP need to be cofinanced. That means that the EU contributes $50-75 \%$ of the money depending on the area classification (e.g. Sachsen-Anhalt is classified target area 1 and receives a share of $75 \%$ from the EU). The remaining amount has to be raised by the individual Länder. Both Länder under investigation utilise the option of obtaining federal funds to reduce their financial burden. The instrument of the 'Common Task for the Improvement of Agricultural Structures and Coastal Protection' (or 'GAK') has been used to co-ordinate Länder policies with those of the federal level. If a Land has opted to offer part of its agri-environmental schemes under the GAK there is an additional federal contribution of $60 \%$. In turn, the federal government determines the funding conditions for measures co-financed under this federal scheme. This structure leads to the complex influence of European, federal and state regulations.

When preparing agri-environmental schemes at state level, not only these regulations on different levels need to be considered but also the interests of different stakeholders. The Länder government strives for a balanced programme. The top agricultural authority develops the programme but cannot decide autonomously. Council regulation 1698 requires close consultations in the implementation of the EAFRD assistance: with competent regional, local and other public authorities, economic and social partners and other bodies representing civil society as well as non-governmental organisations, including environmental organisations (as specified in article 6). Apart from this official requirement the programming authority has an incentive to involve stakeholder groups to have access to the information necessary for scheme development and coordination. Scheme 'development' mainly refers to alterations made to existing schemes rather than formulation of completely new ones. Because the recent programming period is the second continuation of an existing programme initiated in 1992, the administrations minimise their efforts and their risks by adapting their formerly approved programme to the demands of the new European and federal regulations. The challenge is to

\footnotetext{
${ }^{76}$ For an introduction to the issues and tools of assessing environmental problems via indicators see e.g. Piorr, 2003
} 
promote the schemes and to enhance their acceptance, economic efficiency and ecological efficacy by adapting them to local characteristics.

Although the programming process in different Länder may vary in detail, the overall procedure is similar. We can distinguish between a drafting and a coordination phase. There are no formal rules as to who is to be involved at the drafting stage. However, it is common practice for the programming department to involve administrative organisations on the same and on lower levels. They include neighbouring departments within the ministry or relevant departments of other ministries (e.g. environment or conservation departments) as well as advisory bodies (e.g. Landesanstalt für Landwirtschaft, regional offices for agriculture) and Agriculture Agencies (Ämter für Landwirtschaft; in Lower Saxony called Agriculture Chamber, Landwirtschaftskammer). In some cases, experts may be consulted by the administrative authority and influence the process during this phase.

Another set of actors involved are interest groups. The representatives of agricultural associations have frequent contacts with the relevant ministry departments and thus form an agricultural policy network. The involvement of agricultural interest groups is largely informal and non-regulated. This corporatist participation or network corporatism (Wessels, 2000) is characteristic of German politics, in particular in the area of policies dealing with agriculture. In contrast, public participation or citizen participation is uncommon at this level. The selection of who can participate depends on the (actual or anticipated) influence of the association, personal characteristics of the group's representative and the decision of senior staff in the ministry. The Gemeinsame Geschäftsordnung (management and public relation rules) of the ministries only define in broad terms that "relevant stakeholder groups shall be involved". The term comprises major interest groups and top organisations of relevant unions and associations, local interest groups and other parties with the legal right to participate (e.g. Ministerium des Inneren, 1998). All of this input is channelled through the responsible technical departments within the ministry, e.g. the Departments for Nature and Soil Conservation, the Department for Forestry, or the Department for Water and Waste. 


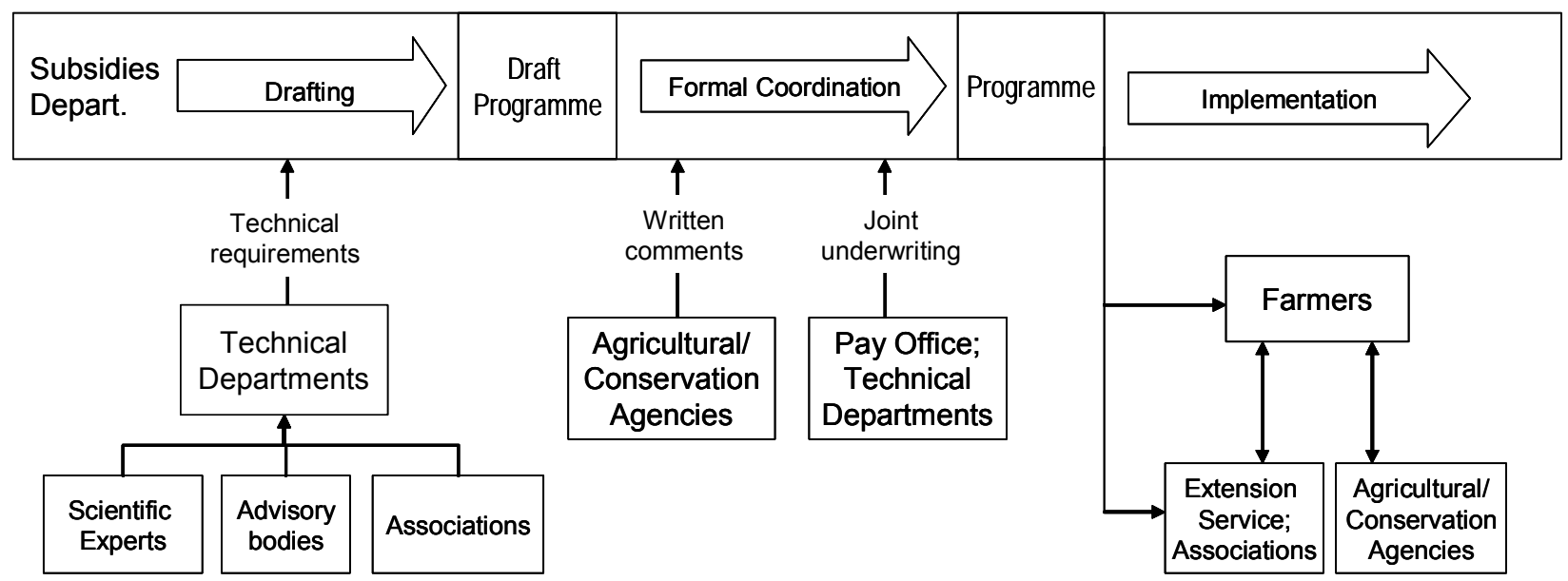

Figure 5.1.: Actors involved in the development and implementation process of agri-environmental programmes on state level (figure by the authors, following Prager, 2006)

The draft of the programme is then presented to the above-mentioned authorities who can now formally comment on the draft. The EU Commission will check whether the draft proposal meets the existing requirements. If so, the programme is agreed upon and notified, marking the beginning of the implementation phase. During the implementation phase the farmers have contacts with the agricultural agency (for some measures also with the local conservation authority), the extension services and their associations. Those interactions are in general the only way that feedback from the farmers can be transported back into the ministry for consideration in future programming processes. Figure 5.1. illustrates the present policy making process.

This apparent top-down approach continues to be the prevailing approach in Germany. Other countries experience a similar situation. For example, Smithers and Furman (2003) judge agri-environmental programmes throughout North America over the past two decades as reflecting a „top-down“ approach. Potter (1998) claims that this strategy has formed the basis for many agri-environmental programmes not only in North America, but also in the European Union over the past three decades. At the same time, attempts are being made to modify or supplement this top-down approach. Examples are the Ontario Environmental Farm Plan Programme (Smithers and Furman, 2003) and the Land-care movement in Australia (Wilson, 2004).

\subsubsection{Conflicting interests}

The process outlined above introduces the different stakeholders into the policy making process. Each of the stakeholders belongs to a group that has particular objectives regarding the output of this process, the agri-environmental schemes. Figure 5.2. assembles some of the 
demands associated with administration, nature conservation, government and farmers.

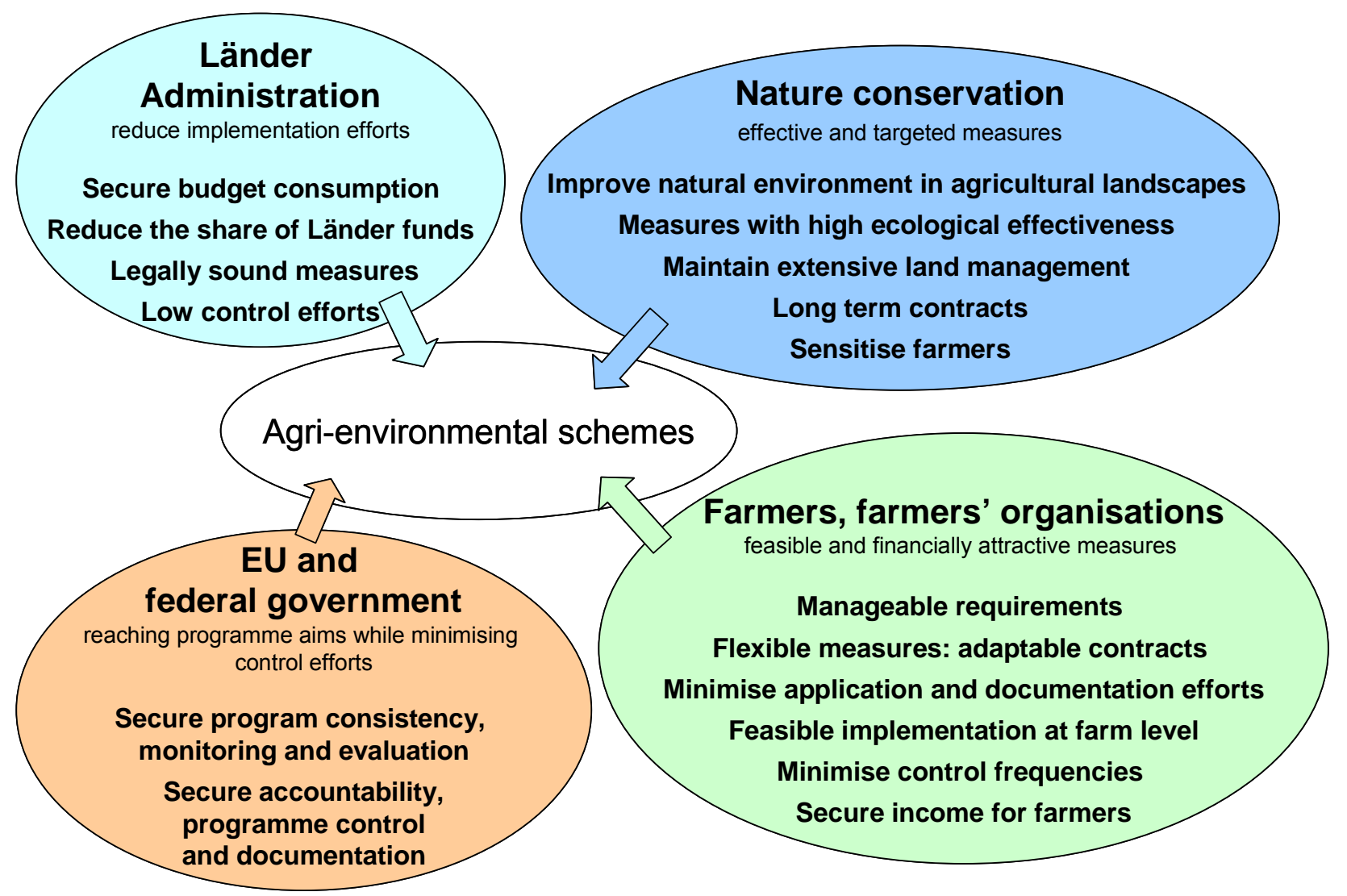

Figure 5.2.: The diverse demands from different stakeholder groups (figure by the authors).

The EU governs the AES by setting a framework and gives Member States the space they need to implement them. In the process of rural development programming the Länder also have to compile the regulations determining AES. The development and implementation of these regulations is carried out in an area of conflict between the following dimensions:

1. AES are directed towards farmers. Farmers participate voluntarily so the programmes must be acceptable for them. This means that they must fit into their every-day farming activities and must compensate costs and losses derived from programme participation.

2. AES aim to improve the state of the environment so the programmes must generate positive ecological effects. The measures must be ecologically effective.

3. AES are funded by money from EU, federal government and Länder sources, so programmes and funds have to be administrated effectively and accountability ensured and demonstrated.

The acceptance of programmes is a prerequisite to achieving any effects at all due to the 
voluntary nature of the schemes. If farmers do not enter schemes, no change in production systems will occur, and no activities for the enhancement of biodiversity will be implemented. If only a small number of farmers sign an agreement, effects will be marginal. A similar effect can be expected, if farmers enrol but do not implement activities in an appropriate way. In order to achieve any measurable effects it is necessary for a critical number of farmers to enrol in the programme and implement the measures accordingly. Farmer uptake of programmes, measured in number of agreements, is sometimes also called farmers“ „,acceptance“ of schemes. A high „acceptance“ is also the goal of policy makers: they want to develop schemes that actually deliver the money to farmers and land managers. Farmers' enrolment is the consequence of many different factors. Yet, it does not automatically imply the individual farmer's acceptance of a programme if acceptance is understood as the successfully completed process of perception, evaluation, trial, and adoption as well as adaptation of an innovation (Prager, 2002). ${ }^{77}$ If a participant approves of the scheme's objectives and is convinced that the chosen practices contribute to these objectives (i.e., he or she is accepting them), the quality of how and the extent to which the practices are implemented will be positively influenced. This in turn has an impact on effectiveness.

The common claim of the Länder administration is that it is responsible for the correct implementation of the EU-framework. In case of misuse or inadequate implementation, the Länder administration carries a considerable financial risk (i.e. if it fails to secure accountability and comply with EU regulations and guidelines the Land has to repay European funds). They also have to regard the ecological efficiency of the measures. Since investigations questioned the ecological effects of the programmes (see e.g. Kleijn et al., 2001, EU-Court of Auditors, 2000, Kleijn and Sutherland, 2003) current and upcoming evaluations always ask for the ecological impact. Administrations spending money through programmes like the AES that should improve the environment have to be able to demonstrate positive impacts.

In times of deregulation, cutbacks of administrations and the crisis of the public budgets, it is essential for the administration to minimise their costs and efforts for the implementation. The so-called transaction costs - the costs for implementation, administration and control of the programme - have to be minimised without neglecting the ecological and political aims.

\subsection{Two Key studies}

Two independently organised and funded studies were carried out, one by a team from the

\footnotetext{
${ }^{77}$ For detailed discussions on the phenomenon of acceptance see Prager (2002, pp. 6-29). Lehmann et al. (2005, pp. 27-30) provide an extensive review of relevant literature.
} 
Humboldt University of Berlin in collaboration with the Ministry of Agriculture and Environment in Sachsen-Anhalt, the other one by researchers from the University of Göttingen in collaboration with a Landcare Association in Lower Saxony.

Although different in their detailed objectives, the overall aim of both projects was similar: Improving communication and participation as an immediate result and increasing the acceptance of agri-environmental schemes in the long run.

The hypothesis on how the relevant aspects influence each other is illustrated in figure 5.3.

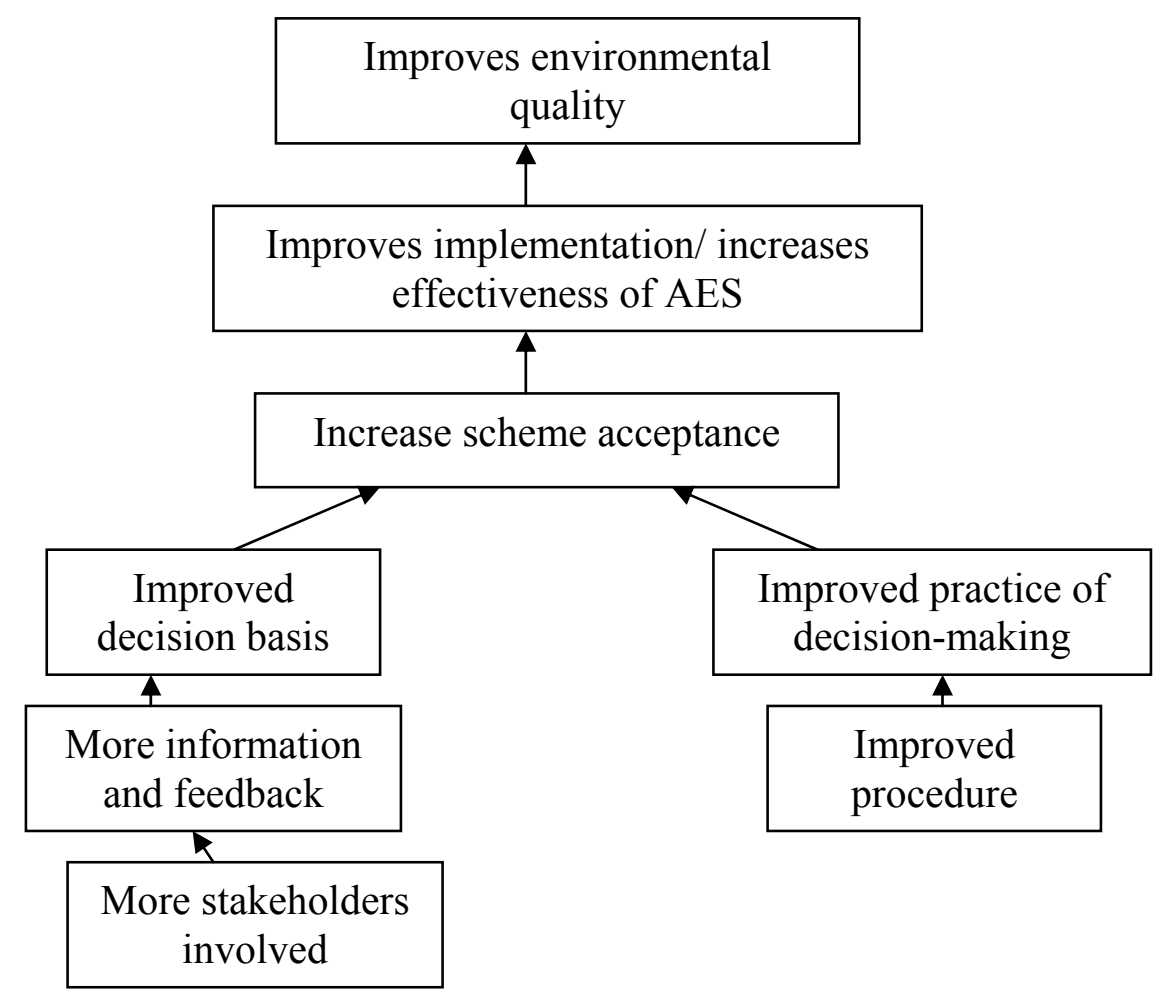

Figure 5.3.: Illustration of Assumptions of the functional chain (figure by the authors).

The authors assume that improved communication and decision-making procedures will ultimately have positive effects on the effectiveness of agri-environmental schemes. Put simply, the authors argue that improved procedures in terms of fairness and transparency which include user feedback about agri-environmental schemes through participation will improve both the information basis and the practice of decision-making processes. This will have favourable effects on the quality of results of those processes, e.g. better schemes. If schemes as well as decision-making procedures are perceived to have improved, farmers' acceptance of schemes will increase. Increased acceptance will improve scheme implementation per se (e.g. prescriptions are implemented according to their intent and less control is 
needed) and thereby increase the schemes' effectiveness. In the long run, this can be expected to contribute to an improved environmental quality.

The objective was to provide decision support to administrative decision makers through collecting (local) information through the participatory process (Lower Saxony, SachsenAnhalt) and a mathematical model for budgetary decision-making (Sachsen-Anhalt). Part of this objective was to ensure a transparent policy-making process.

The following table 5.1. contains the essence of the two projects. The tabular listing facilitates comparisons between the studies. For detailed reports of the case study in Sachsen-Anhalt see Prager and Nagel (2004), Kirschke et al. (2004) and Kirschke et al. (2005).

Table 5.1.: Overview of two Key studies:

\begin{tabular}{|c|c|c|}
\hline & Sachsen-Anhalt & “Bördeproject Lower Saxony” \\
\hline \multicolumn{3}{|l|}{ Project information } \\
\hline $\begin{array}{l}\text { Concerns decision- } \\
\text { making processes on }\end{array}$ & $\begin{array}{l}\text { The level of federal state, } \\
\text { Ministry of Agriculture and the } \\
\text { Environment of Sachsen-Anhalt state } \\
\text { (MLU) }\end{array}$ & $\begin{array}{l}\text { Local to regional level } \\
\text { Börderegion }{ }^{78} \text { in Lower Saxony } \\
\text { state }\end{array}$ \\
\hline Research approach & $\begin{array}{l}\text { Action research, aimed at developing } \\
\text { problem-oriented and context-adapted } \\
\text { solutions based on the cooperation } \\
\text { between research and 'real life' }\end{array}$ & $\begin{array}{l}\text { Demonstration/pilot project } \\
\text { organised by the local stakeholders, } \\
\text { assisted and supported by socio- } \\
\text { economic research (action research) }\end{array}$ \\
\hline Research team from & $\begin{array}{l}\text { Institute of Agricultural Economics at } \\
\text { Humboldt University of Berlin }\end{array}$ & $\begin{array}{l}\text { Centre for Agriculture and the } \\
\text { Environment (ZLU) at University } \\
\text { of Göttingen }\end{array}$ \\
\hline $\begin{array}{l}\text { Role of the research } \\
\text { team }\end{array}$ & $\begin{array}{l}\text { Facilitate the organisation of work- } \\
\text { shops, manage the mathematical } \\
\text { model, documentation of process: } \\
\text { Researchers have no influence on final } \\
\text { decisions regarding budget allocation }\end{array}$ & $\begin{array}{l}\text { Collect and arrange scientific } \\
\text { information, being part of the } \\
\text { project organisation committee, } \\
\text { documentation of process: Involved } \\
\text { in decision making only as part of } \\
\text { the project organisation committee. } \\
\text { Facilitate the project advisory } \\
\text { committee meetings and implement } \\
\text { their decisions }\end{array}$ \\
\hline Initiator of the process & Research team & $\begin{array}{l}\text { ZLU and Landcare Association } \\
\text { (LA) comprising various local } \\
\text { stakeholders }\end{array}$ \\
\hline $\begin{array}{l}\text { To launch the project, } \\
\text { first contacts were } \\
\text { made with }\end{array}$ & $\begin{array}{l}\text { MLU Secretary of State and the head } \\
\text { of subsidies department }\end{array}$ & $\begin{array}{l}\text { Division officer for organic farming } \\
\text { and AES in the Lower Saxony } \\
\text { Ministry of Agriculture (ML) }\end{array}$ \\
\hline The process was & Research team and ministry officials & The LA in tight cooperation with \\
\hline
\end{tabular}

\footnotetext{
${ }^{78}$ Börderegion is an intensively farmed region with loess soil, in short called Börde. There are several Börderegionen in Germany.
} 


\begin{tabular}{|c|c|c|}
\hline organised by & & the researchers \\
\hline Involved parties & $\begin{array}{l}\text { Head of subsidies department, head of } \\
\text { division, one staff member as contact } \\
\text { person } \\
\text { Workshop participants: the above and } \\
\text { staff of advisory bodies and } \\
\text { neighbouring departments, representa- } \\
\text { tives of conventional farmers' union, } \\
\text { organic farmers' organisations }\end{array}$ & $\begin{array}{l}\text { Development of project idea: LA, } \\
\text { staff member of ML and research } \\
\text { team } \\
\text { Collaboration with: LA, agricultural } \\
\text { chamber, farmers union, County } \\
\text { administration, conservation } \\
\text { organisation, local agricultural } \\
\text { extension services, hunter's } \\
\text { organisation }\end{array}$ \\
\hline Initial situation & $\begin{array}{l}\text { Programming in the hands of ministry, } \\
\text { irregular and informal hearings with } \\
\text { farmer's unions/ associations initiated } \\
\text { by the ministry; no environmental or } \\
\text { Landcare Associations involved, } \\
\text { occasional involvement of neighbour- } \\
\text { ing departments and local agriculture } \\
\text { agencies }\end{array}$ & $\begin{array}{l}\text { Ministry and stakeholders were } \\
\text { interested in an example of AES for } \\
\text { intensively farmed regions. LA was } \\
\text { already established and had } \\
\text { connections to some stakeholders. } \\
\text { Involvement of local environmental } \\
\text { organisations and the county } \\
\text { administration in policy making via } \\
\text { the LA. }\end{array}$ \\
\hline \multicolumn{3}{|l|}{ Project history } \\
\hline Issue & $\begin{array}{l}\text { Reaching a decision - consensual if } \\
\text { possible - on the re-distribution of } \\
\text { funds for agri-environmental pro- } \\
\text { grammes for the next planning period; } \\
\text { stakeholders acknowledged no } \\
\text { additional conflicts }\end{array}$ & $\begin{array}{l}\text { Developing a new agri- } \\
\text { environmental measure (prescrip- } \\
\text { tion) for the intensively farmed } \\
\text { region in collaboration with farmers } \\
\text { and other stakeholders }\end{array}$ \\
\hline Duration & $\begin{array}{l}\text { Preparation and organisation of two } \\
\text { workshops between April and July } \\
2003 \\
\text { Frequent contacts mainly to ministry } \\
\text { staff since then }\end{array}$ & $\begin{array}{l}\text { Project started in February } 2003, \\
\text { since then continuous working } \\
\text { relationship }\end{array}$ \\
\hline $\begin{array}{l}\text { Steps (Participatory } \\
\text { methods and scientific } \\
\text { methods used) }\end{array}$ & $\begin{array}{l}\text { 2 preparatory meetings in April- } \\
\text { May 2003, } \\
2 \text { workshops in June-July } 2003 \\
\\
\text { Workshops featuring facilitated } \\
\text { discussions, application of a } \\
\text { mathematical model for priority } \\
\text { setting and budgeting, documenta- } \\
\text { tion of workshop results and dis- } \\
\text { tribution to all participants }\end{array}$ & \begin{tabular}{|l}
- \\
Development of project idea \\
together with local stakeholders \\
2003 first launch of new \\
measure \\
2004 slight modification of \\
measure and re-launch \\
- Organisation of project steering \\
group and project advisory \\
board meetings and expert input \\
into process \\
Project advisory board: enables \\
continuous, informal and formal \\
contacts between LA, other \\
stakeholders, scientists and the \\
ministry. \\
Irregular informal reports about \\
the project given to the ministry \\
by the board
\end{tabular} \\
\hline
\end{tabular}




\begin{tabular}{|c|c|c|}
\hline & $\begin{array}{l}\text { Stakeholder and conflict analyses; } \\
\text { analyses of communication net- } \\
\text { works within the agricultural ad- } \\
\text { ministration, } \\
\text { Results of analysis, stakeholder } \\
\text { feedback, and recommendations } \\
\text { provided to ministry, }\end{array}$ & $\begin{array}{l}\text { Parallel socio-economic } \\
\text { research, analysis of: } \\
\text { inhibiting factors for up- } \\
\text { take/acceptance of measures, } \\
\text { costs and stakeholder structure, } \\
\text { process and results } \\
\text { Collection of information and } \\
\text { feedback for project board, } \\
\text { political and administrative } \\
\text { decision makers }\end{array}$ \\
\hline Input research team & $\begin{array}{l}\text { No direct money transfer, } \\
\text { input of resources by providing } \\
\text { scientific expertise for mathematical } \\
\text { model and workshop facilitation }\end{array}$ & $\begin{array}{l}\text { Payment for measure demonstra- } \\
\text { tions (farmer grants), } \\
\text { resources and scientific expertise }\end{array}$ \\
\hline Input stakeholders & $\begin{array}{l}\text { Ministry provided meeting rooms, sent } \\
\text { invitations }\end{array}$ & Time and manpower \\
\hline Output & $\begin{array}{l}\text { Short-term funding strategy docu- } \\
\text { mented in the minutes of the work- } \\
\text { shops }\end{array}$ & $\begin{array}{l}\text { An additional measure in the Lower } \\
\text { Saxony's AES. }\end{array}$ \\
\hline Selected outcomes & $\begin{array}{l}\text { Learning effects: thinking in objectives } \\
\text { and contributions; } \\
\text { Stakeholders understood the ministry's } \\
\text { incentives for cut-backs in conserva- } \\
\text { tion measures (Vertragsnaturschutz); } \\
\text { Officials failed to integrate consensus } \\
\text { in subsequent paper } \\
\text { Stakeholders disappointed and lost } \\
\text { interest, } \\
\text { Continued contacts between research- } \\
\text { ers-ministry, but no follow-ups could } \\
\text { be arranged so far }\end{array}$ & $\begin{array}{l}\text { Stable cooperation und communica- } \\
\text { tion at local level. Risk-minimising } \\
\text { by testing an agri-environmental } \\
\text { measure in a restricted area before } \\
\text { introducing them state-wide. } \\
\text { Participants learned about the } \\
\text { significance of local-long term } \\
\text { dedicated work, the value of the LA } \\
\text { and acknowledged the need for a } \\
\text { conservation/ environmental } \\
\text { advisory system. }\end{array}$ \\
\hline $\begin{array}{l}\text { Relevant for EAFRD- } \\
\text { Regulation Article... }\end{array}$ & $\begin{array}{l}\text { Art. } 6 \text { "Partnership" in preparation, } \\
\text { implementation, monitoring and } \\
\text { evaluation of the rural development } \\
\text { programmes }\end{array}$ & $\begin{array}{l}\text { Art. } 61 \mathrm{ff} \text { (in section 4) "Local } \\
\text { action groups" as local public- } \\
\text { private partnerships }\end{array}$ \\
\hline
\end{tabular}

\subsection{Discussion of lessons learned}

Two different participatory approaches were introduced in the previous chapter. Participation is generally viewed as positive and beneficial. However, the fact that participation is not an end in itself is illustrated in the apparent contradiction noted by Illsley (2003): Despite the increasing opportunities for citizens to influence decision-making processes, this has not resulted in greater public satisfaction with decisions taken. Citizens' perceptions and assessment of decision-making processes can be sub-divided into two distinct fields: perception of distributive justice (the fairness of the outcomes of decisions) and procedural justice (fairness 
within the decision-making process). Lind and Tyler (1988) found that fair processes are frequently more significant to citizens than fair outcomes.

With this in mind, our discussion is not focussed on the output of the respective processes, i.e. the resulting agri-environmental programmes or budget allocation. The effectiveness and efficiency of programme prescriptions is subject to various evaluation studies in the literature (Kleijn, 2001, Carey et al., 2005, Carey et al., 2003 or the midterm-reviews of the rural development programmes e.g. ML, 2004). We are concerned with the procedure of participatory approaches. The scientific component of both approaches provided for the analysis of the respective participatory processes. Survey methods used were interviews with the involved actors and observation. In addition, various methods for judging the trustworthiness of the data such as participant checking and triangulation by multiple sources and methods were applied (see Pretty et al., 1995, p.59). The analyses allow the discussion of the involved parties, the level of participation and the impact.

Numerous publications are looking at what makes participation successful. Some authors have compiled factors important for successful conservation projects (Brendle, 1999, Knierim and Liebe, 2003) or variables that are likely to affect successful organisational change (White, 2001), while others have listed criteria for fair participation (Illsley, 2003) or conditions that interactive policy making has to meet if it is to contribute effectively to the resolution of complex social issues (Driessen et al., 2001). Many of the key aspects in these publications have also emerged in the course of the two studies and may be considered essential factors for successful participation processes. We have grouped the significant factors into those that are relevant for the preparation and for the facilitation of participatory processes. We subsequently discuss the problems encountered with the respective approaches as well as the positive effects.

\subsubsection{Premises and preparation}

\section{Interest in the issue}

All actors must have an interest in the issue. This is sparked by an anticipated added value - a benefit - for all parties. ${ }^{79}$ Potential incentives could be a share of the decision-making power or access to information: either from the ministry accessing stakeholder information or vice versa. An alternative is the existence of an urgent issue demanding a solution. If this is not the case, parties will not be willing to invest their resources, be they time, money or manpower. In Lower Saxony, all stakeholders saw a potential win-win situation even though not all of

\footnotetext{
${ }^{79}$ It could also be sparked by a perceived threat to distributive justice. In that case the value is a reduced threat.
} 
them could realise their initial ideas (e.g. the conservationists could not expect a measure with high ecological impact). The project happened to develop at the right time and the researchers met a convenient situation. There were also sufficient incentives for actors in Sachsen-Anhalt, even though they lay in different areas for different stakeholders: the associations participated to get information and have yet another chance to make their views heard (apart from the regular hearings); the ministry participated to convince the farmers' representatives of what the future political strategy would have to be, utilising the involvement of scientist to support the ministry's envisaged strategy.

The different type of interest is also shown by who initiated the process. In Lower Saxony, the Landcare Association and its associated stakeholders previously had an idea about what they wanted. Scientific support and the formation of a project were most welcome. It is likely that the process will continue without the researchers' support. In Sachsen-Anhalt, however, it was the research team approaching the ministry. The interest ceased after the first round of workshops. It became obvious that the state-level approach depends on:

- researchers' good contacts with key actors within the ministry;

- those key actors being interested in and in favour of the approach; and

- the ministry's political climate supporting stakeholder involvement.

\section{Decision alternatives}

There must be decision alternatives. With regard to the legal framework and the political situation there needs to be leeway for decision-makers. In Lower Saxony, a compromise could be negotiated between agricultural and conservation interests. Alternatives in SachsenAnhalt were restricted: due to reduction of state funds, there were only limited alternatives for shifting the money in other ways because of legal and accountability requirements. The chances for setting up new programmes were minimal due to issues of bias and the amount of time granted for workshops. Those issues are closely related to the criteria for fair participation stated by Illsley (2003): A fair process is dependent on non-biased decision-makers, who should not be influenced by self-interest. Any decision-making space is influenced by the people involved. Ultimately, the decision in Sachsen-Anhalt was in the hands of ministry officials, who appeared to be biased with a prefixed strategy in mind. In Lower Saxony, bias was not a dominant issue. This may be due to the extensive overlap, in this specific context, of aims between ministerial decision-makers and stakeholders. 


\section{Who is an appropriate participant?}

Before initiating a participatory process, the selection of participants is an important question, although the question of who is "relevant" may emerge repeatedly during the process. Information about relevant actors (Driessen et al. 2001 call this "analysis of dependency relations") also enables a process manager to select the "appropriate" participants for a planned process. In addition, knowledge of the situation, actors and conflicts are essential for both approaches. If there is no time allowed for those preparatory steps, both workshops and collaboration process are not likely to be successful. Gathering stakeholders "around a table" may hold problems especially when the local groups have not cooperated before or relationships are burdened by past conflicts. Hidden conflicts as well as hidden agendas impede the collaboration of all actors.

\subsubsection{Structuring and facilitating the participatory process}

\section{Trust}

Trust in the parties and the process is essential. Trust may be both a factor influencing the process but also a result of the process (see below). The local parties in Lower Saxony enjoyed to a certain extent an atmosphere of trust which made it easy to start the project and get together the stakeholders. In Sachsen-Anhalt, due to previous experiences in their working relationships, the parties lacked trust in each other. However, they were confident in the process as the researchers were deemed to take the role of objective outsiders and ensure a fair process.

\section{Transparency}

There should be a high degree of transparency. In Lower Saxony, the project was initially mainly focused on the development of an agri-environmental measure and to promote this extensification by means of flowering strips. The project mainly used the existing structures of the LA so the stakeholders were clear about the next steps, the way decisions were made and what the aim of the collaboration was. Transparency in the decision-making process could also be ensured in Sachsen-Anhalt. The mathematical model showed the underlying assumptions for budgetary decisions clearly, facilitated the discussion and encouraged participants to state their interests and concerns. 


\section{A dedicated person}

To keep the process going, a dedicated person, team or organisation is needed (see Brendle, 1999). In both cases a large part of the focus on this came from the research team (project planning and implementation and direct or indirect investments). The 'local cooperation level' that already existed in Lower Saxony supported the approach. Participants already had or developed a sense of 'project ownership'. From the socio-economic analysis carried out by the research team we infer that the process would have started without the involvement of researchers. The local stakeholders of the LA had clear ideas what to do. However, the researchers strengthened the process of developing agri-environmental measures by their access to and overview of studies and reports about similar projects. Due to the LA, the support of the agricultural chamber and the positive experiences for the stakeholders, the process and the collaboration structures have a good chance to persist. The German Association for Landscape Conservation (Deutscher Verband für Landschaftspflege, DVL) as the umbrella organisation for the German Landcare Associations is a strong leader and can give interested local stakeholders important advice and assistance. Often, institutions such as advisory boards or Biological Stations or Conservation Stations also work without scientific assistance.

For Sachsen-Anhalt, the dedicated person appeared in the form of the Secretary of State and the department head who agreed to the application of the approach but it was the research team that initiated and pursued the cooperation.

\subsubsection{Problems encountered}

This section discusses the problems encountered with the initiation and management of the participatory approaches. We will point out what additional input was required and which aspects remained outside the scope of the approaches.

\section{Input and resources}

An additional effort associated with participation is caused by the input needed from all parties involved: the coordinating organisation needs resources to facilitate meetings and coordinate the process; the participating groups need to designate participants and invest time and manpower. The financial input can be limited if the process is planned and managed well.

\section{Participant motivation}


Keeping the stakeholders motivated is a challenge for the process manager. Participatory processes have their limits when is comes to the issue of who holds the final decision-making power. Laws and regulations on higher levels (e.g. the federal or the European level) restrict the possible outcomes. Drawbacks among the participants can be caused if advice or suggestions are not integrated, if regulations do not match local demands or situations ${ }^{80}$ and unexpected or unwanted changes in relevant legislation. A locally organised participation process may also have disadvantages for the federal state administration. This model needs a more complex AES and at least a limited transfer of decision power from the upper to the local level. To motivate the local participants the AES regulations must allow modification by local authorities or institutions. In North Rhine Westphalia for example the AES is menu-driven: the local authority can vary measures and spatially restrict them in order to adapt them to local demands. Also manpower and financial resources by the state must be accessible for the local level organisation. And finally the state must guide, support, control and audit the local organisations, their outcomes and processes.

\section{Organised vs. unorganised stakeholders}

The state-level approach has only been tested when involving organised stakeholders, i.e. representatives of farmers' unions and associations. Although we believe that the approach is adequate for the involvement of individual farmers and other stakeholders we acknowledge the fact that the ministry is not used to dealing with citizens that are not members of associations and has no incentive to do so.

\section{Adequacy of a mathematical model}

A mathematical model as employed in Sachsen-Anhalt relies on researchers' expertise to run the model, facilitate the data input process and explain the implications of the modelling results. The application of a mathematical model may also cause technical problems, for example if there is changes in the composition of the participating group, extra time needs to be spent on acquainting the newcomers to the model. Ensuring constant attendance, however, is impossible due to the numerous other activities and tasks of both ministry staff and farmers' representatives. A shortcoming of the model-based approach is that not all types of problems can be solved by discussing budgetary priority setting. The mathematical model is best suited to a situation where political programmes are already defined and known to all

\footnotetext{
${ }^{80}$ Example: Schemes require a maximum width for flowering strips of 3 meters. However, the farmers machinery is 5 meters wide.
} 
stakeholders. In other situations, preparatory steps are essential, such as designing programmes or single measures (as done in Lower Saxony) or to discuss stakeholders' objectives.

\section{Linkages to policy issues}

An essential requirement for success of participatory processes - be they located at the local or the state level - is the linkages to real policy issues. The outcome or, even better, the whole process needs to have links to related decision-making processes. Researchers may seek out those linkages but cannot force them. Integration of the resulting agri-environmental measure into the state's menu of AES was ensured by the ministerial representative in Lower Saxony. In Sachsen-Anhalt, the ministry officials failed to integrate parts of the workshop results into the department's agri-environmental funding strategy and did not explain their reasoning to the involved stakeholders which lead to a loss of confidence and interest on the side of farmers' representatives. Certain features of the state-level approach point to a "top-down" layout with only few opportunities of integrating ideas bottom-up: Researchers plan the process and final decision making power rests in the hands of ministry officials. The chances to expand bottom-up elements are determined by ministry officials - if researchers "ask to much" the collaboration is at risk. This observation was asserted by a fellow researchers' experience.

\subsubsection{Benefits}

This section highlights the benefits and impacts of the introduced participatory approaches that occurred (refer also to 'output and outcome' in the table 5.1. in part 5.4.). Amongst the positive effects we find direct as well as indirect effects.

\section{Improved information feedback}

In Sachsen-Anhalt, information has been fed back to the ministry in a structured way. Participants were considered experts in the field of AES. The assessment of each programme's contribution to the agri-environmental objectives by means of a round of expert valuation provides realistic information, since there are no objectively verifiable means to quantify these contributions of single schemes. This information would not have been made explicit without the workshops. Any information gathered and results of the workshops whether in case of consensus or disagreement, have been documented for future reference. Similarly, the involvement of local stakeholders made it possible to collect and bundle 
information for the decision-making process that would otherwise remain spread among the various participants.

\section{Improved communication}

Both approaches create opportunities for the open exchange of ideas and disputes. The participants' way of thinking can be altered and collective learning processes initiated. Bringing together local stakeholders, as happens in Lower Saxony, initiated a discussion process. Communication starts or can be improved with respect to objectivity and frequency. Improved communication can generate or improve trust and understanding between the parties. It is a complex task to explain the function of the mathematical model to participants. This takes time that might be spent in other ways. However, once participants have understood the basic mechanisms in the model, the model is capable of increasing the efficiency of the discussion. It reveals which aspects of a problem are important and which ones can be neglected.

\section{Increased acceptance of agri-environmental schemes}

In both case studies, participants have made positive remarks on the process itself as well as on the results of that process. Farmers' representatives are able to communicate their contentment to their constituencies. A positive reaction is likely to increase acceptance at the organisation basis. Stakeholders do not simply take the issue back to their organisation but together they can also promote and advertise this issue, targeting for example the local politicians and farmers. In this way they can raise the acceptance of potentially interested farmers as well as the local people's acceptance towards the farmers' agri-environmental activities. Because stakeholders' ideas have been directly integrated into a new agrienvironmental measure, the acceptance of this measure is likely to be significantly higher than that of similar measures developed without participation.

The topic of agri-environmental development is set on the regional agenda. This is essential because acceptance depends on the perception of an issue and knowing about function and objectives of an instrument. Only if the farmers, conservationists and administrations talk about the agri-environment and the programmes fostering it can they develop a common interest in the topic. This first knowledge: the easy access to information and to be pointed to the programmes is the basis for getting interested and later would be crucial to accepting and 
implementing agri-environmental schemes.

\section{Building networks}

Stakeholder involvement can make the participants' networks and resources available. The stakeholders can generate common aims and projects. They may identify fields of cooperation, find innovative solutions to the identified problems and generate win-win situations. If the stakeholders have generated projects and worked together on the issue of agrienvironmental improvement they can develop a long term perspective for the issue. To a limited degree it can be decoupled from interventions and funding of the administration. This corresponds with Moseley (2003) who highlights 'attracting new partners' and 'independence from formal institutions' as the most important results of rural development groups.

\section{Types of participation}

There are different ways of grouping participatory approaches: for example according to their legal basis, the political level they are employed at, the stakeholders involved, duration, intensity, timing within a planning process, suitability for conflict resolution or according to the main functions and purposes they serve. Examples for classifications are given by Bischoff et al. (1995) but shall not be discussed here, as we intend to demonstrate the change of 'quality' in the way different stakeholders have been involved during the case studies. In an attempt to describe both the type of participation existing prior to involvement of the researchers and the type of participation implemented via the participatory approaches, two different typologies appeared meaningful: The older one is Arnstein's ladder of participation (Arnstein, 1969) consisting of 8 rungs. The higher on the ladder a participatory approach is, the greater the extent of citizens' power in determining the end product of public policy. Pretty et al. (1995) compiled a typology of participation describing 7 types. They represent seven ways in which the term participation can be interpreted and used. ${ }^{81}$ For a better understanding, these two typologies are included in the appendix.

In both cases, the level of participation changed towards a stronger involvement of stakeholders in decision-making processes. In Sachsen-Anhalt, a standard situation including

\footnotetext{
${ }^{81}$ Recent typologies do not depart widely from the two selected ones, e.g. Tress et al. (2005) describe the different levels stakeholder participation can be realized at. Referring to different roles of stakeholders in a research process, Tress et al. distinguish the lowest level 'being informed', level 2 'consultation' (and providing data and input), level 3 'being involved' and the highest level 'steering/controlling the process'.
} 
hearings with the representatives of farmers' organisations can be classified by 'Participation in giving information' (type 2 according to Pretty et al., 1995) and 'Informing' (rung 3 on Arnstein's ladder of participation). Due to the project this has changed to 'Participation by consultation' (type 3 according to Pretty et al., 1995) with elements of 'Placation / Consultation' (rung 4/5 of Arnstein's ladder of participation).

The initial situation in Lower Saxony can be described as 'Passive participation' (type 1) because local people had the option whether to enrol or not in the AES. There were no linkages between those local level stakeholders the project was concerned with and the ministry (e.g. local group of farmers union, Kreisbauernverband). However, there are also hearings in the ministry with the state level representatives (e.g. farmer's union on state level, Landesbauernverband) similar to Sachsen-Anhalt. The classification according to Arnstein is difficult in this case: The situation could be considered 'Non-participation' (rung 1 'Manipulation' or 2 'Therapy') with weak elements of 'Informing'. This changed to 'Interactive participation' (6) following the typology of Pretty and 'Partnership' (6) according to Arnstein. The stakeholders developed and tested the measures and reported to the ministry. The ministry adapted central comments.

\subsection{Conclusions and Recommendations}

The two main goals of agri-environmental schemes as part of the rural development programmes are to provide an income support to farmers and to achieve positive effects on environmental and natural resources. The upper (policy-making) level and the lower (policyimplementing) level are mutually dependent. The framework of measures and conditions of participation is set by policy makers. However, due to the schemes' voluntary nature, their implementation depends on the individual farmer or land manager. So it is those individuals who ultimately decide the success of the programme - if no contracts are signed, no prescriptions are carried out and no impacts will be achieved. The implementation of the EAFRD regulation is envisaged through "close consultation", synonymous with "partnership".

In the light of these requirements, experiences with two participatory approaches are presented in this paper. One of the approaches addresses the local level, the other the state level. Both levels cannot be viewed separately; processes and outcomes on one level will influence processes and outcomes on the other level. The approaches give examples for the way participatory processes can be organised. An important feature of the approaches is that they have not been conducted according to a standard procedure but remain flexible to be adapted 
to the actors involved, to their respective problems and positions as well as their civic capacities and personal demands ${ }^{82}$. Both approaches are aiming at an increased rate of information exchange, transparent decision-making processes and, in the long run, improved agri-environment programmes that elicit a higher acceptance both by farmers and by society as a whole. They have proven to contribute to an improved participatory process if improved is understood as "moving up" on the participation ladder.

We claim that the approaches can be utilised simultaneously and to promote interfaces for ideas and information moving upward and downward. An "advisory committee" or "regional partnership" on the lines of the one formed in Lower Saxony is characterised as an intermediary organisation. The local approach is better suited to integrate local stakeholders and mediate between their individual interests. A goal- and problem-oriented discussion is more likely to take place at the local level because this is the level of programme implementation: the level where implementation problems become visible first. Ideas and concepts of local stakeholders can be coordinated or integrated and thus represent regions at a higher governmental level. For example, this organisation could come up with regionally adjusted agrienvironmental measures to be discussed on the state level. The committee will still be representing the interests of its members; however, those positions would have already been negotiated on a lower level. An arrangement such as this supports the committee's position as an influential partner in bargaining processes about budgetary questions at state level.

The advantage of this approach is that it can help to avoid arguments between government and interest groups that are removed from reality and often not focussed on problem resolution but deal with questions of power, self-profiling and self-referenced actions. The intermediary organisation represents not only agricultural interests of the farmer lobby groups but comprises various regional interests.

Including a wider range of interests on the local level and representing those interests via an intermediary organisation at state-level policy making is one way to meet requirements of the EAFRD regulation. If a two-step approach such as the one suggested cannot be implemented yet, because for example there are no regional competences, funding for the establishment of local agri-environmental programmes is lacking or links to the relevant stakeholders are missing, a focus on the improvement of decision-making processes at state level has advantages. Under the present legal requirements administrative bodies at the state level are responsible for shaping agri-environmental programmes. Their decisions affect the whole

\footnotetext{
82 civic capacities (social capital, expresses the level of experience people have with participatory planning/action) and personal demands (on time, level of commitment required by the approach)
} 
state. Improved programmes at this level are expected to have positive impacts throughout the state. However, in contrast with the case study of the state level approach presented, the circle of participants in this approach can and should be extended to encompass "the most representative partners" also in the "environmental sphere" (Art 16). From the methodological view this does not pose problems. The mathematical model can focus discussions and support budgetary decision-making regardless of who the participants are. However, it is the officials in the ministry that have yet to recognise participatory processes not only as placation in a strategy of tokenism but as a chance to improve the state's political programmes. Facilitated discussions should not only be used as "a selling performance" for predetermined ideas but as an opportunity of equal involvement and voice for all participants. The issue of who are the relevant actors that should be included remains controversial and cannot be universally answered. Where to draw the line will greatly depend on who will be affected by a decision taken and who is considered a "relevant actor". Some claim that an analysis of the major dependency relations provides an answer. We believe that this is part of the flexibility that marks a participatory approach: anybody interested and affected should be given the opportunity to participate.

\subsection{Appendix}

Appendix 5.1: Arnstein's Ladder of Participation (Arnstein 1969), adapted

\begin{tabular}{|l|l|l|}
\hline 8 & Citizen Control & \multirow{3}{*}{ Participation } \\
\hline 7 & Delegated Power & \multirow{3}{*}{ Tokenism } \\
\hline 6 & Partnership & \\
\hline 5 & Placation & \\
\hline 4 & Consultation & \multirow{2}{*}{ Non-participation } \\
\hline 3 & Informing & \\
\hline 2 & Therapy & \\
\hline 1 & Manipulation & \\
\hline
\end{tabular}

Appendix 5.2: Forms of participation (Pretty et al., 1995), adapted

\begin{tabular}{|l|l|l|}
\hline 1 & $\begin{array}{l}\text { Passive } \\
\text { participation }\end{array}$ & $\begin{array}{l}\text { People are being told what is happening. It is a unilateral announcement by } \\
\text { an administration or project management without listening to people's } \\
\text { responses. The information being shared belongs only to external profession- } \\
\text { als. }\end{array}$ \\
\hline 2 & $\begin{array}{l}\text { Participation in } \\
\text { information giving }\end{array}$ & $\begin{array}{l}\text { People participate by answering questions posed by researchers using } \\
\text { questionnaires or surveys. People do not have the opportunity to influence } \\
\text { procedures or outcomes, as the findings are neither shared nor checked for } \\
\text { accuracy. }\end{array}$ \\
\hline 3 & $\begin{array}{l}\text { Participation by } \\
\text { consultation }\end{array}$ & $\begin{array}{l}\text { People participate by being consulted, and external agents listen to views. } \\
\text { These external agents define both problems and solutions and may modify } \\
\text { these in the light of people's responses. }\end{array}$ \\
\hline 4 & Participation for & People participate by providing resources such as labour, in return for food, \\
\hline
\end{tabular}




\begin{tabular}{|l|l|l|}
\hline & material benefits & $\begin{array}{l}\text { cash or other material incentives. It is very common to call this 'participa- } \\
\text { tion' yet people often have no stake in deciding about the processes and in } \\
\text { extending activities when incentives end. }\end{array}$ \\
\hline 5 & $\begin{array}{l}\text { Functional } \\
\text { participation }\end{array}$ & $\begin{array}{l}\text { People participate by forming groups to meet predetermined objectives } \\
\text { related to the project. These institutions tend to be dependent on external } \\
\text { initiators and facilitators, but may become self-dependent. }\end{array}$ \\
\hline 6 & $\begin{array}{l}\text { Interactive } \\
\text { participation }\end{array}$ & $\begin{array}{l}\text { People participate in joint analysis, which leads to action plans and the } \\
\text { formation of new local institutions or the strengthening of existing ones. It } \\
\text { tends to involve interdisciplinary methodologies that seek multiple objectives } \\
\text { and make use of systematic and structured learning processes. These groups } \\
\text { take control over local decisions, and so people have a stake in maintaining } \\
\text { structures or practices. }\end{array}$ \\
\hline 7 & $\begin{array}{l}\text { Institutionalized } \\
\text { participation }\end{array}$ & $\begin{array}{l}\text { People participate by taking initiatives independent of external institutions. } \\
\text { They develop contacts for resources and technical advice they need, but } \\
\text { retain control over how resources are used. Consultation and joint decision- } \\
\text { making is a must for project implementation. }\end{array}$ \\
\hline
\end{tabular}

\subsection{References}

Arnstein, S. R., 1969. A Ladder of Citizen Participation. Journal of the American Institute of Planners 35 (4), 216-224, quoted according to Choguill, M.B.G., 1996. A Ladder of Community Participation for Underdeveloped Countries. Habitat International 20 (3), 431-444.

Bischoff, A., Selle, K., Sinning, H., 1995. Informieren, Beteiligen, Kooperieren: Kommunikation in Planungsprozessen: Eine Übersicht zu Formen, Verfahren, Methoden und Techniken. Dortmund: Dortmunder Vertrieb für Bau- und Planungsliteratur.

Brendle, U., 1999. Musterlösungen im Naturschutz - Politische Bausteine für erfolgreiches Handeln. Landwirtschaftverlag, Münster.

Carey, P.D., Short, S., Morris, C., Hunt, J., Priscott, A., Davis, M., Finch, C., Curry, N., Little, W., Winter, M., Parkin, A., Firbank, L.G., 2003. The multi-disciplinary evaluation of a national agri-environment scheme. Journal of Environmental Management 69, 71-91.

Carey, P. D., Manchester, S.J., Firbank Les G., 2005. Performance of two agri-environment schemes in England: a comparison of ecological and multi-disciplinary evaluations. Agriculture, Ecosystems and Environment, Volume 108 (3) 178-188

Driessen, P.P.J., Glasbergen, P., Verdaas, C. 2001. Interactive policy-making - a model of management for public works. European Journal of Operational Research 128, 322-337.

EU-Court of Auditors, 2000. Special Report No 14/2000 on "Greening the CAP" together with the Commission's replies. Official Journal of the European Communities, Volume 43. http://www.eca.eu.int/audit_reports/special_reports/docs/2000/rs14_00en.pdf (11.1.06).

Feindt, P.H., Newig, J., 2005. Politische Ökonomie von Partizipation und Öffentlichkeitsbeteiligung im Nachhaltigkeitskontext. In: Feindt, P.H., Newig, J. (Eds.) 2005. Partizipation, Öffentlichkeitsbeteiligung, Nachhaltigkeit., Metropolis, Marburg, pp.9-42.

Freese, J., H.-H. Steinmann 2006. Improving Agri-environmental schemes: Improving the institutional delivery of agri-environmental schemes via local action groups (in press).

Freese, J., Steinmann H.-H., 2005. Ergebnisse des Projektes „,Randstreifen als Strukturelemente in der intensiv genutzten Agrarlandschaft Wolfenbüttels“. Diskussionsbeitrag 0503 des Institutes für Agrarökonomie, Universität Göttingen, Göttingen.

http://wwwuser.gwdg.de/ uaao/margg/discussionpaper0503.pdf (13.1.06).

Grafen, A., Schramek, J., 2000. Germany: complex agri-environmental policy in a federal system. In: Buller, H., Wilson, G.A. and Höll, A., (Eds.), 2000. Agri-environmental policy in the European Union. Ashgate, Aldershot, pp.119-143.

Hanley, N., Whitby, M., Simpson, I., 1999. Assessing the success of agri-environmental policy in the UK. Land Use Policy 16, 67-80.

Illsley, B.M., 2003. Fair participation - a Canadian perspective. Land Use Policy 20, 265-273. 
Kirschke, D., Häger, A., Jechlitschka, K., Wegener, S., 2004. Priority setting for rural development: An interactive PC-based programming approach. In: Petrick, M., Weingarten, P. (Eds.). The Role of Agriculture in Central and Eastern European Rural Development: Engine of Change or Social Buffer?: IAMO, Halle (Saale), Studies on the Agricultural and Food Sector in Central \& Eastern Europe 25, pp. 398-412, also: www.iamo.de/dok/sr_vol25.pdf (7.3.06).

Kirschke, D.; Häger, A.; Jechlitschka, K., Wegener, S., 2005. Co-financing implications for regional policymaking: A case study for the agri-environmental programme in Saxony-Anhalt. Sutra Working Paper No 5. Humboldt-University of Berlin.

http://www.agrar.hu-berlin.de/sutra/workingpaper/wp5/wp5.pdf (7.3.06).

Kleijn, D., Brendse, F., Smit, R., Gilissen, N., 2001. Agri-environment schemes do not effectively protect biodiversity in Dutch agricultural landscape. Nature 413, pp. 723-725.

Kleijn, D., Sutherland, W.J., 2003. How effective are agri-environment schemes in conserving and promoting biodiversity? Journal of Applied Ecology 40: 947-969.

Knierim, A., Liebe, F., 2003. Gemeinsame Prozessgestaltung als Weg zu erfolgreichem Naturschutz. Natur und Landschaft 78 (8), pp. 354-359.

Lehmann, P., Schleyer, C., Wüstemann, H., Drechsler, M., Hagedorn, K., Wätzold F., 2005. Promoting the Multifunctionality of Agriculture, Forestry, and Rural Areas - Design and Implementation of Public Policies in Germany. UFZ-Diskussionspapier 12/2005. http://www.ufz.de/data/Disk_Papiere_2005-122648.pdf (4.1.2006).

Lind, A.E., Tyler, T.R., 1988. The Social Psychology of Procedural Justice. Plenum Press, New York.

Marggraf, R., 2003. Comparative Assessment of Agri-environment Programmes in Federal States of Germany. Agriculture, Ecosystems and Environment 98, pp. 507-516.

Ministerium des Inneren des Landes Sachsen-Anhalt, 1998. Beschluß der Landesregierung über die Gemeinsame Geschäftsordnung der Ministerien - Allgemeiner Teil. Ministerialblatt LSA Nr.3/1998 vom 21.1.1998.

ML - Ministry of Agriculture, Lower Saxony, 2003. Halbzeitbewertung von Proland Niedersachsen. Braunschweig. http://www1.ml.niedersachsen.de/proland/Aktuelles.htm (21.12.05).

Moseley, M.J. (ed.) 2003. Local Partnerships for Rural Development. The European Experience. CABI Publishing, Oxon.

Ostrom, E. 1990. Governing the Commons: The Evolution of Institutions for Collective Action. Cambridge University Press, Cambridge.

Ostrom, E., Gardner, R., Walker, J., 1994. Rules, Games, and Common-Pool Resources. University of Michigan Press, Ann Arbor.

Piorr, H-P., 2003. Environmental policy, agri-environmental indicators and landscape indicators. Agriculture, Ecosystems \& Environment 98, pp. 17-33.

Potter, C., 1998. Conserving nature: agri-environmental policy and change. In: Ilbery, B. (Ed.). The Geography of Rural Change. Addison Wesley Longman, Harlow, pp. 85-105.

Prager, K. 2006. Participatory decision-making on agri-environmental programmes - A case study from SachsenAnhalt (Germany). Land Use Policy (in press).

Prager, K. and Nagel, U.J., 2004. Communication processes in agro-environmental policy development and decision-making - Case study Sachsen-Anhalt. Sutra Working Paper No 2. Humboldt-University of Berlin.

http://www.agrar.hu-berlin.de/sutra/workingpaper/wp2/wp2.pdf (7.3.2006).

Prager, K., 2002. Akzeptanz von Maßnahmen zur Umsetzung einer umweltschonenden Landbewirtschaftung bei Landwirten und Beratern in Brandenburg. Margraf, Weikersheim.

Pretty, J. N., Guijt, I., Thompson, J., Scoones, I., 1995. A Trainer's Guide for Participatory Learning and Action. International Institute for Environment and Development, London.

Renn, O., Webler T., Wiedemann P. (eds.), 1995. Fairness and Competence in Citizen Participation: Evaluating Models for Environmental Discourse. Kluwer Academic, Dordrecht, pp. 17-33.

Smithers, J. and Furman, M., 2003. Environmental farm planning in Ontario: exploring participation and the endurance of change. Land Use Policy 20, 343-356.

Tress, B., Tress, G., Fry, G., 2005. Defining concepts and the process of knowledge production in integrative 
research. In: Tress, B, Tress, G., Fry, G., Opdam, P. (eds.). From landscape research to landscape planning: aspects of integration, education and application. Springer, Heidelberg, pp. 13-26. http://library.wur.nl/frontis/landscape_research/02_tress.pdf (7.3.2006).

Wessels, B., 2000. Condemned to co-operate. Mitbestimmung international edition 8, pp. 47-50.

White, S. S., 2001. Public participation and organizational change in Wisconsin land use management. Land Use Policy 18, 341-350.

Wilhelm, J. 1999. Ökologische und ökonomische Bewertung von Agrarumweltprogrammen - Delphi-Studie, Kosten-Wirksamkeits-Analyse und Nutzen-Kosten-Betrachtung. Europäische Hochschulschriften V/2542, Peter Lang, Frankfurt/Main.

Wilson, G.A., 2004. The Australian Landcare movement: towards 'post-productivist' rural governance? Journal of Rural Studies 20, pp. 461-484.

Wilson, G.A. and Wilson, O.J., 2001. German Agriculture in Transition - Society, Policies and Environment in a Changing Europe. Palegrave, London. 


\section{Kapitel 6}

\section{A Fuzzy Tale - Die kleinen Leute von Borka ${ }^{83}$}

„Eine der tückischsten und unheilvollsten Ei-
genschaften wissenschaftlicher Modelle ist ihre
Fähigkeit, die Wirklichkeit zu schlagen und sich
an ihre Stelle zu setzen. Oft dienen sie als
Scheuklappe, indem sie die Aufmerksamkeit auf
einen übertrieben engen Bereich beschränken. “

Erwin Chargaff

(1995, S. 192 f. Naturwissenschaft als Besessenheit. In: Das Feuer des Heraklit. München: dtb, 2. Auflage)

Es gab irgendwann einmal ein Volk von kleinen glücklichen Leuten. Sie nannten sich die Bork und lebten in einem fernen Land, das von ihnen auf den Namen Borka getauft worden war. Dort war das Leben sehr angenehm, man lebte in kleinen Dörfern, umgeben von saftigen Weiden, unberührten Wäldern und sanften Hügeln. Überall war das Rauschen des kleinen, sauberen Quellflusses zu hören, der durch das Land floss und die Dörfer verband. Die Borks waren glückliche Wesen, die gerne feierten, hilfsbereit und mit sich und der Welt zufrieden waren. Am meisten aber liebten sie es, sich einander kleine, warme und weiche Pelzchen zu schenken. Immer wenn sich zwei Borks begegneten, tauschten sie die neuesten Geschichten aus und überreichten sich zum Abschied weiche warme Pelzchen. Dies bedeutete: „Ich mag dich! “, und darum war es das Schönste, kleine Pelzchen geschenkt zu bekommen oder selber zu verschenken. Es war ein wundervolles Gefühl, über das warme flauschige Pelzchen zu streichen. Man konnte förmlich spüren, was der andere damit sagen wollte. Man fühlte sich anerkannt und geliebt und wollte dem anderen auch sofort etwas Gutes tun.

Einmal trug es sich aber zu, dass ein besonders grüblerischer und ängstlicher Bork glaubte, dass das Tauschen von Pelzchen die Borks in naher Zukunft ins Verderben stürzen würde. Er zog sich deshalb in eine Hütte im Wald zurück und verbrachte sein Leben damit, zu verstehen, was die Welt ist und wie alles zusammenwirkt, um so das Zusammenleben der Borks

\footnotetext{
${ }^{83}$ Veröffentlicht als Euler, M. und Freese, J. (1996): A Fuzzy Tale - Die kleinen Leute von Borka. Homo oeconomicus (Adecco, München) XII (2), 1996, S.277-280. Frei nach der Kettenbriefgeschichte „Die kleinen Leute von Swabedoo“ (Verfasser unbekannt, veröffentlicht im Verlag Partisch \& Röhling).
} 
vernünftiger zu organisieren, als das die Pelzchen seiner Überzeugung nach je könnten.

Als er endlich nach vielen Jahren des Grübelns den letzten Federstrich unter seine Gedanken gemacht hatte, hüpfte der mittlerweile alt gewordene Bork vor Freude und lief fröhlich ins nächste Dorf.

Als er dem ersten Bork begegnete und dieser ihm lächelnd ein Pelzchen überreichen wollte, wies er dieses zurück und sagte: „Sei vernünftig und nicht so großzügig mit deinen Pelzchen, sonst gehen sie dir noch aus.“ Der kleine Bork schaute ihn mit großen fragenden Augen an und verstand nicht.

„Sieh mal, das Tauschen von Pelzchen mit Fremden ist doch viel zu unsicher. Woher willst du wissen, ob du von mir eine entsprechende Gegenleistung erhältst?“ Der kleine Bork war so verwirrt, dass er gar nicht darüber nachdachte, dass das überhaupt nicht passieren konnte: Für jedes Pelzchen, das er verschenkte, bekam er ja eines zurück und hatte somit ein unerschöpflichen Vorrat. Der alte Bork ließ ihn weinend und verzweifelt zurück und eilte zum Bürgermeister. Mit ihm wollt er sofort das Nötige besprechen. Der Bürgermeister rief gleich seinen Kollegen aus den anderen Dörfern zu einer Besprechung zusammen, und sie diskutierten die Ideen des alten Bork bis spät in die Nacht.

Währenddessen saß der verwirrte und traurige Bork vor seinem Haus und grübelte über die Worte des alten Borks nach. Schon bald kam ein guter Freund vorbei, mit dem er schon viele Pelzchen getauscht hatte: „Was bist du so unglücklich? Es ist so ein schöner Abend!“ Er gab dem Unglücklichen ein Pelzchen und lud ihn zu sich zum Abendessen ein. Darauf erwiderte der traurige Bork: „Nein, nein, behalte dein Pelzchen lieber. Sonst hast du irgendwann nicht mehr genug davon, wenn du zu allen immer so großzügig bist. Und wer weiß, vielleicht wirst du dann von den anderen ausgenutzt und erhältst gar keine Gegenleistung!“ Der Freund schüttelte nur den Kopf und verstand die Welt nicht mehr. Er verabschiedete sich leise und stapfte bekümmert nach Hause. An diesem Abend hörte man noch öfters, dass ein Bork zu dem anderen sagte: „Nein danke, behalte deine Pelzchen lieber für dich!“‘

Als die Nacht hereinbrach, hatte sich in Borka eine seltsam bedrückende und kalte Atmosphäre eingeschlichen.

Am folgenden Tag ließen die Bürgermeister alle Borks wissen, wie sie die Zukunft von Borka sicherer und schöner machen wollten. Als erstes wurde eine Pelzchenzählung durchgeführt, um festzustellen, wieviele Pelzchen es gab. Als dies getan war, ließen sie verkünden, dass es nur eine begrenzte Menge von Pelzchen gab, genauso wie der alte Bork es vorausgesehen und berechnet hatte. Dies hätte über kurz oder lang zu Hunger, Leid und Raub in Borka geführt. 
Doch mit den neuen vernünftigen Verteilregeln des alten Borks, die ab sofort gelten sollten, würde es niemals mehr dazu kommen können, denn es wurde bestimmt, dass alle Dinge einen Wert haben, und dieser mit Pelzchen ausgedrückt werden soll.

Bald darauf begannen die ehemals freundlichen und hilfsbereiten Borks sich darüber zu streiten, wieviel Pelzchen eine Übernachtung oder eine Mahlzeit genau kosten sollte. Man begann zu handeln und zu feilschen. Ohne Bezahlung war kein Bork mehr bereit, etwas für den anderen zu tun. Ja, es kam sogar zu vereinzelten Fällen von Pelzchenraub. Der alte Bork fühlte sich in seiner Analyse bestätigt, denn was er befürchtet hatte, war eingetreten: Die Borks waren egoistisch und gierig. Ohne seine Verteilregeln wäre alles zusammengebrochen.

In Borka aber wurde das Leben immer unwerter zu leben. Man traute sich an Abenden, an denen man sich früher im Park traf, feierte und warme weiche Pelzchen tauschte, nicht mehr allein auf die Straße, man misstraute einander und verkehrte nur noch mit anderen Borks, wenn man etwas von ihnen wollte. Das Schlimmste aber passierte einige Zeit später: Alle Borks wurden griesgrämig und krank.

Nun war alles Glück aus Borka gewichen und der alte Bork verschwand wieder im Wald ... 


\section{Fazit}

Der wissenschaftliche Naturschutz bzw. die Naturschutzforschung, deren Ziel die Bereitstellung wissenschaftlicher Beiträge zum Schutz der Arten, Biotope und Prozesse ist, fußt überwiegend auf der wissenschaftlichen Biologie. Methodisches Ziel ist die Bereitstellung fachspezifischer Bewertungsmethoden, die Suche nach Bioindikatoren zur Beschreibung des Zustandes der Natur und die Erarbeitung reproduzierbarer Methoden (Plachter 1991: 9). Dabei ist Naturschutz unbestritten in die gesellschaftlichen und politischen Prozesse eingebunden, und da vom Naturschutz selbst eine handlungsorientierte und praxisgerechte Bereitstellung der wissenschaftlichen Erkenntnisse angestrebt wird, gewinnen gesellschaftswissenschaftliche Disziplinen zunehmend an Bedeutung. Heute liefern die vorher oft zur Begründung und Legitimierung des Naturschutzes herangezogenen Disziplinen der Ökonomie, Ethik und Psychologie (Plachter 1991: 4) zunehmend relevante Beiträge für die Findung und Umsetzung der Naturschutzziele.

In dieser Arbeit wurden einige Beiträge, die die ökonomische Denktradition bzw. ökonomische Teildisziplinen zu Naturschutzfragen beitragen können, dargestellt. Auf theoretischer Ebene kann die Ökonomie, basierend auf dem Verhaltenskonzept des methodologischen Individualismus Vorraussagen bzw. Abschätzungen für menschliches Verhalten treffen bzw. Strategien entwickeln, wie - d. h. mit welchen Instrumenten - Verhalten umweltgerecht gesteuert werden kann. Dazu wurde in Kapitel 1 die erweiterte Kapitaltheorie vorgestellt, die mit ihrem Konzept des Identitäts-, Human- und Sozialkapitals in der Lage ist, auch jenseits des Marktes eingesetzt zu werden. Gleichzeitig wurde in diesem Rahmen eine ökonomische Interpretation der Anreize zur Entstehung sozialer Interaktionen und somit sozialer Phänomene dargestellt. Die Vorführung der spezifischen umweltökonomischen Betrachtung der öffentlichen Güter in Kapitel 1 stellt die sozialen und individuellen identitätsbildenden Aspekte als zentrale Anreize zur individuellen Beteiligung an der Bereitstellung von Gemeinschaftsgütern heraus. Es bleibt aber eine Aufgabe der Zukunft, dieses erweiterte ökonomische Verhaltensmodell mit den vielen ökonomischen Teildisziplinen abzugleichen und es an gesellschaftlichen Phänomenen zu eichen. 
Die in Kapitel 3 vorgestellten Konzepte des volkswirtschaftlichen Gesamtnutzens (total economic value) und der Zahlungsbereitschaftsanalyse stellen dem gegenüber sehr konkret ein Instrument zur Messung der gesellschaftlich relevanten Aspekte des Naturschutzes, nämlich dessen Nutzen für und Wertschätzung durch den Menschen, im Rahmen der naturschutzfachlichen Eingriffsregelung bereit. Hier liegt es an der Praxis und insbesondere an den Naturschutzverwaltungen, ob sie in einer entsprechenden Erweiterung der Eingriffsregelung nicht nur einen zusätzlichen Nutzen sehen, sondern ihnen auch die Vorteile einer stärkeren Berücksichtigung gesellschaftlicher Bewertungen vermittelt werden können.

Um Naturschutzziele innerhalb der Gesellschaft durchsetzbar zu machen, müssen sie Niederschlag in Handlungsempfehlungen finden, die Institutionen und jeder Einzelne in der täglichen Praxis berücksichtigen können. $\mathrm{Zu}$ dieser gesellschaftlichen und individuellen Dimension des Naturschutzes trägt die politische Ökonomie, denen die Betrachtungen der Kapitel 2 bis 5 zuzuordnen sind. Dabei stellt Kapitel 2 durch die Analyse der historischen Prozesse und Konflikte zwischen der Landwirtschaft als Landnutzer und dem Naturschutz die gemeinsame - auf mühsamen Wegen erreichte - Diskussionsbasis dar, die erst eine Grundlage für eine gemeinsame Problemlösung bilden konnte. Dabei kann für die Zukunft - denn dieser gemeinsame Weg des partnerschaftlichen Problemlösens ist gerade erst begonnen worden -gelernt werden, wie Streit vermieden und Energie gespart werden kann. Denn es muss klar sein, dass Partizipation neuer Gruppen an Entscheidungsprozessen eine Machtneuordnung darstellen und dieser Prozess daher sehr fragil ist.

Da die Wertschätzung der Menschen für die Natur einerseits der Antrieb für Naturschutz und

gleichzeitig eine seiner stärksten, weil nachvollziehbarsten Begründungen ist (Köhler/ Preiß 2000), wird in Kapitel 3 vorgeschlagen, diese im Rahmen der Naturbewertung messbar zu machen. Demonstriert wird der Einsatz der Zahlungsbereitschaftsanalyse im Rahmen der sogenannten naturschutzfachlichen Eingriffsregelung, einer der meistgenutzten und wichtigsten Naturschutzinstrumente. Dem konkreten Naturschutz dient auch die Analyse konkreter Projekte in Kapitel 4 und 5 und die Herausarbeitung der Hemm- und Erfolgsfaktoren kooperativer Naturschutzprojekte.

\section{Literatur}

Plachter 1991: Naturschutz. Stuttgart: Gustav Fischer.

Köhler, B, Preiß, A 2000: Erfassung und Bewertung des Landschaftsbildes. In: Informationsdienst Naturschutz Niedersachsen $1 / 2000$. 


\section{Veröffentlichungen (Stand September 2006)}

\section{$\underline{\text { Paper }}$}

Prager K., J. Freese (accepted with major revisions): Stakeholder involvement in agrienvironmental policy making - Success factors for participatory approaches from two German case studies. Journal of Environmental Management.

Groth, M., J. Freese (2006): Ausschreibungen - ein neues Instrument des Vertragsnaturschutzes. Natur und Landschaft Jg. 81, Heft 4, S.202-205.

\section{Buchkapitel, Beiträge in Sammelbänden}

Freese, J., I. Bräuer (2005): Zahlungsbereitschaftsanalysen im umweltrelevanten Bewertungsverfahren. Die Eingriffsregelung als Beispiel. In: Marggraf, Bräuer, Fischer, Menzel, Stratmann, Suhr: Ökonomische Bewertung bei umweltrelevanten Entscheidungen, Metropolis. S.307-332.

Freese, J., H.-H. Steinmann (2006): Improving the institutional delivery of agrienvironmental schemes via local action groups. In: B.C. Meyer (Ed.) 2006: Sustainable Land Use in Intensively used Agricultural Regions. Landscape Europe. Alterra report No 1338. Wageningen, pp. 119-126.

Freese J., A. Richter gen. Kemmermann (2005): Agrarumweltprogramme: einzelbetriebliche Beratung und regionales Management als Erfolgsfaktoren. In Elsen, T. von „Einzelbetriebliche Naturschutzberatung - ein Erfolgskonzept für mehr Naturschutz in der Landwirtschaft. Beitrage der Tagung von 6.-8. Oktober 2005 in Witzenhausen. FiBEL Deutschland, Witzenhausen. S.127-136.

Freese J., C. Rüffer (2005): Kooperativer Naturschutz in der Kulturlandschaft. In Feindt, Newig: Partizipation, Öffentlichkeitsbeteiligung, Nachhaltigkeit. Perspektiven der politischen Ökonomie. Metropolis. S.250-271.

H.-H. Steinmann, J. Freese, B. Gerowitt (2005) Zur pflanzlichen Artenvielfalt in Ackerbaulandschaften - Situation, Einflussgrößen, Perspektiven. In: R. Forster, E. Bode, D. Brasse: Das Bienensterben im Winter 2002/03 in Deutschland, Bundesamt für Verbraucherschutz und Lebensmittelsicherheit, Braunschweig, S. 30-41.

Freese, J. (2004): Naturschutz in der intensiv genutzten Agrarlandschaft. In: H. Korn, U Feit: Treffpunkt Biologische Vielfalt 4, Interdisziplinärer Forschungsaustausch im Rahmen des Übereinkommens über die biologische Vielfalt. Bundesamt für Naturschutz. Bonn - Bad Godesberg 2004. S.137-141. 
Euler, M., J. Freese, K. Briedes (2000): Jenseits von Rente und Vollbeschäftigung: Die Neue Arbeitsgesellschaft. In: D. Dettling (Hrsg.): Deutschland ruckt! Die junge Republik zwischen Brüssel, Berlin und Budapest. Frankfurt/M. POD.

Freese, J., M. Euler (1996): Die Kleinen Leute von Borka. homo oeconomicus XII(2), S.277280.

\section{Working paper}

Freese, J., H.-H. Steinmann (2005): Ergebnisse des Projektes „Randstreifen als Strukturelemente in der intensiv genutzten Agrarlandschaft Wolfenbüttels“. Diskussionsbeitrag 0503 des Inst. f. Agrarökonomie, Universität Göttingen, Juni 2005. 64 S. Göttingen. http://www.gwdg.de/ uaao/margg/marggraf/team/janfreese/discussionpaper0503.pdf (19.8.2006).

\section{Poster und Abstract im Tagungsband}

Freese, J. (2006): Jahrestagung der GeWiSoLa „Unternehmen im Agrarbereich vor neuen Herausforderungen“, 5.-7. Oktober 2005 in Göttingen. Posterpräsentation: Multifunktionalität durch kooperativen Agrarumweltschutz verwirklichen. Abstract in: Umwelt- und Produktqualität im Agrarbereich. 45. Jahrestagung der GeWiSoLa, Band 41, 2006, (in Druck)

Freese, J. (2005): Anforderungen an zukünftige Agrarumweltprogramme - Ergebnisse aus dem Projekt „Lebensraum Börde“. Abstract zum Poster in: Umwelt- und Produktqualität im Agrarbereich. 44. Jahrestagung der GeWiSoLa Band 40, 2005, S.551-552.

Freese, J. (2004): Working on the implementation is more promising than demanding a reformulation of the CAP. Talk at annual conference of the German ecological society 2004, 13-17.9.2004, Giessen. Abstract: Proceedings of the gfö, Vol 34 (2004) p.331. 


\section{Danksagung}

Zum gelingen dieser Arbeit haben über einen langen Zeitraum hinweg sehr viele verschiedene Menschen beigetragen.

Ich bedanke mich bei Prof. Marggraf für die Betreuung meiner Arbeit und die vielen inhaltlichen Diskussionen.

Bei Prof. Theuvsen und Prof. Spiller bedanke ich mich für die übernehme der Korreferate.

Ich danke Prof. Marggraf und Dr. Steinmann für die Möglichkeit der Mitarbeit im Projekt „Lebensraum Börde“. Im Rahmen dieses Projektes hatte ich intensiv Gelegenheit, die praktischen Seiten der Landwirtschaft und des Agrarumweltschutzes kennen zu lernen und vielfältige empirische Untersuchungen durchzuführen.

Unseren Projektpartnern in der Region Braunschweig/ Wolfenbüttel, insbesondere Herr Meier vom Landvolkverband Braunschweiger Land und Herrn Borchers von der Bezirksstelle Braunschweig der Landwirtschaftskammer Niedersachsen, gilt der Dank für die erfolgreiche Durchführung unseres Projektes.

Für fachliche Diskussionen und Ko-Autorenschaften bedanke ich mich bei Dr. Holger Bergmann, Ursula Stratmann und Dr. Tobias Hellenbroich, Anne Richter gen. Kemmermann, Chrsitina Rüffer, Katrin Prager, Dr. Mark Euler, Dr. Anke Fischer, Dr. Benedikt Herrmann, Dr. Horst Steinmann, Prof. Dr. Bärbel Gerowitt, Dr. Hermann Hondong, Markus Groth und Dr. Ingo Bräuer.

Unschätzbar war für mich das herzliche Umfeld in Göttingen und die freundliche und produktive Atmosphäre im Zentrum Landwirtschaft und Umwelt und in der Abteilung Umwelt- und Ressourcenmanagement. Besonders gerne erinnere ich mich an Dr. Thomas zum Felde, Dr. Christina Rüffer, Dr. Holger Bergmann, Anne Richter gen. Kemmermann, Susanne Menzel, Ingke Rachor, Oliver Corell, Sebastian Klimek und Sandra Venghaus.

Für die gemeinsame freundschaftliche Zeit bedanke ich mich bei Cornelia Claußen, Sven-Erik Engmann und Julia Lange.

Bei Linda Junge bedanke ich mich für die Unterstützung in der Endphase und ihr anspornendes und aufmunterndes da sein.

Meinen Eltern, meiner Schwester und Dr. Mark Euler gilt mein Dank für die langjährige unermüdliche Unterstützung.

Diese Arbeit wurde von der Deutschen Bundesstiftung Umwelt im Rahmen des Projekteverbundes Lebensraum Börde unterstützt. 


\section{Lebenslauf}

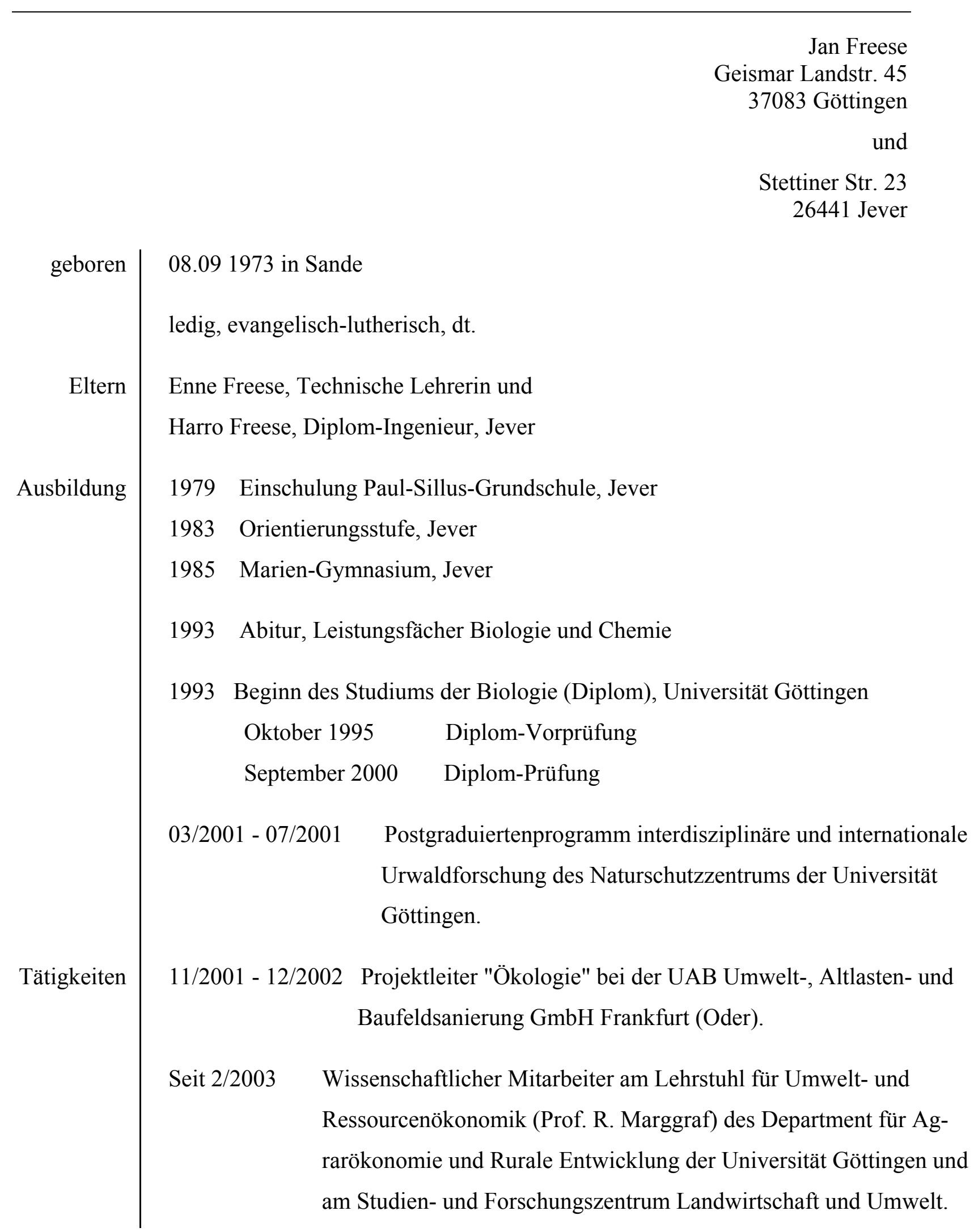

